\title{
Down-Stream molecular Responses to Volatile Signaling in Roots
}

\author{
Dissertation \\ for the award of the degree \\ "Doctor rerum naturalium" \\ of the Georg-August-Universität Göttingen \\ within the doctoral program \\ "Molecular Biology of Cells" \\ of the Georg-August-University School of Science (GAUSS) \\ submitted by \\ Mareike Jakobi \\ from Hildesheim, Germany
}

Göttingen 2020 



\section{Thesis Advisory Committee}

Prof. Dr. Andrea Polle

Department of Forest Botany and Tree Physiology, Büsgen-Institut,

Georg-August-Universität Göttingen

Prof. Dr. Gerhard Braus

Department of Molecular Microbiology and Genetics, Institute for Microbiology and Genetics, Georg-August-Universität Göttingen

Prof. Dr. Jörg-Peter Schnitzler

Research Unit Environmental Simulation,

Helmholtz Zentrum München

\section{Members of the Examination Board}

Referee: $\quad$ Prof. Dr. Andrea Polle

Department of Forest Botany and Tree Physiology, Büsgen-Institut, Georg-August-Universität Göttingen

$2^{\text {nd }}$ Referee: Prof. Dr. Jörg-Peter Schnitzler

Research Unit Environmental Simulation,

Helmholtz Zentrum München

\section{Further Members of the Examination Board}

Prof. Dr. Gerhard Braus

Department of Molecular Microbiology and Genetics, Institute for Microbiology and Genetics, Georg-August-Universität Göttingen

Prof. Dr. Ivo Feußner

Department of Plant Biochemistry, Albrecht von Haller Institute, Georg-August-Universität Göttingen

PD Dr. Till Ischebeck

Department of Plant Biochemistry, Albrecht von Haller Institute, Georg-August-Universität Göttingen

Prof. Dr. Kai Heimel

Department of Molecular Microbiology and Genetics, Institute for Microbiology and Genetics, Georg-August-Universität Göttingen

Date of Oral Examination: $11^{\text {th }}$ of November 2020 



\section{Affidavit}

Hereby, I confirm, that the dissertation "Down-Stream molecular Responses to Volatile Signaling in Roots" has been written independently and no other sources and aids were used than quoted. 



\section{Table of Contents}

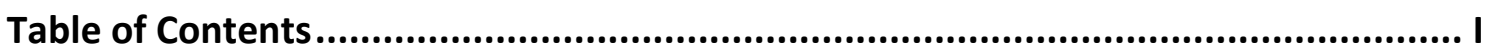

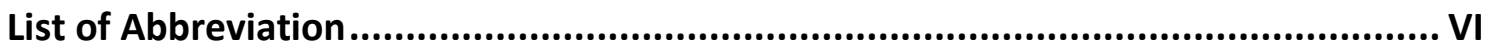

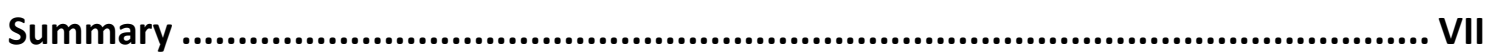

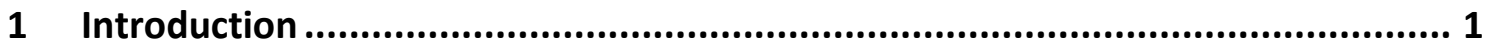

1.1 Fungal derived volatiles and their effects on plants ..................................... 1

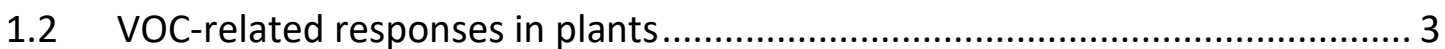

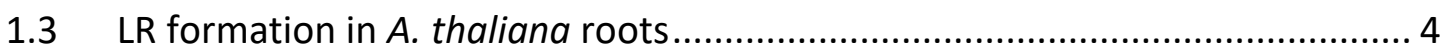

1.4 Influence of abscisic acid, ethylene and jasmonic acid on LR formation .......... 6

$1.5 \quad$ Further factors that influence LR formation ................................................ 9

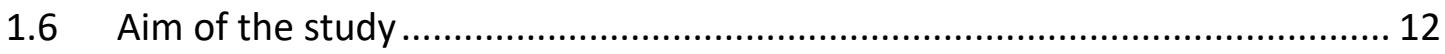

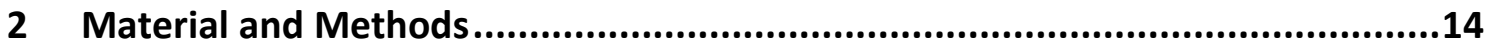

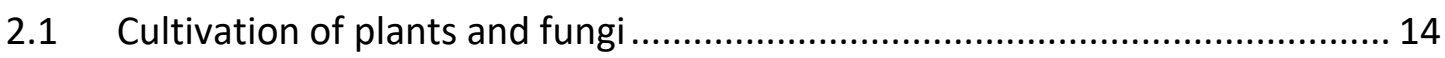

2.1.1 Growth conditions of Arabidopsis thaliana .......................................... 14

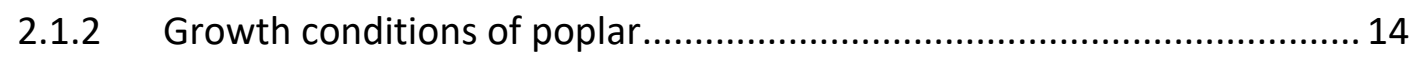

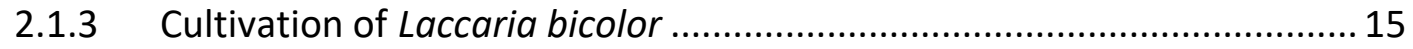

2.1.4 Exposure of $A$. thaliana to $L$. bicolor volatiles ...................................... 15

2.1.5 Exposure of $P . x$ canescens to $L$. bicolor volatiles ................................. 19

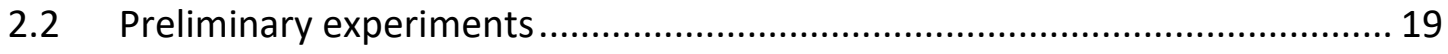

2.2.1 Influence of fungal age on A. thaliana LR density .................................. 19

2.2.2 Comparison of aluminum tray and cellophane foil for grown L. bicolor on

A. thaliana LR formation.

2.2.3 Comparison of $A$. thaliana growth in Phyto agar and Gelrite at different $\mathrm{pH}$ values.

2.2.4 Effect of Farnesyl pyrophosphate ammonium on L. bicolor and A. thaliana growth 20

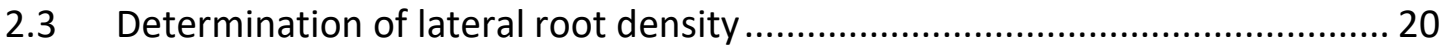

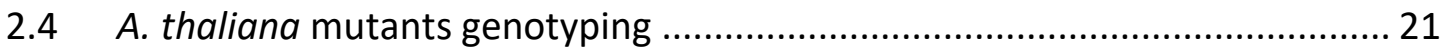

2.5 Identification of $A$. thaliana cysteine rich receptor-like kinase (CRK) homologues in Populus trichocarpa. 
2.6 Screens of $A$. thaliana mutants potentially involved in volatile-induced $L R$

formation .24

2.6.1 Screen of cysteine-rich receptor like kinases (CRKs) and per45 and per59..

2.6.2 Screen of germin-like protein 3 (GLP3) mutants and strigolactone affected mutants .25

2.7 Analysis of the pGATA23::n/s-GFP-GUS line as a marker for LR development 25

2.8 Determination of volatile response dose and time.....................................26

2.8.1 Determination of volatile response dose................................................26

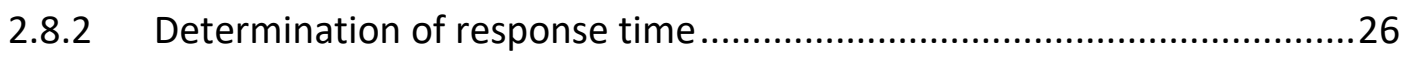

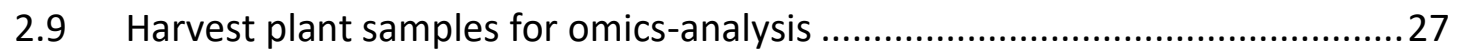

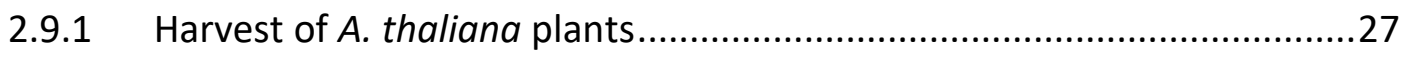

2.9.2 Harvesting of Populus $x$ canescens plantlets ........................................28

2.10 Molecular biological methods and in silico analysis......................................29

2.10.1 DNA extraction of $A$. thaliana leaf samples ..........................................29

2.10.2 RNA extraction and cDNA synthesis...................................................30

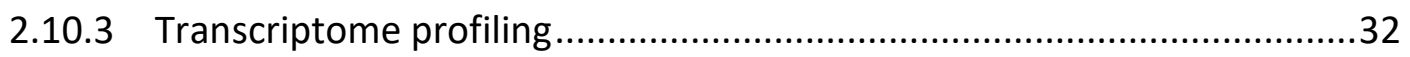

2.10.4 Analysis of transcriptome data with MapMan ......................................36

2.10.5 Identification of VALR homologous genes in A. thaliana and

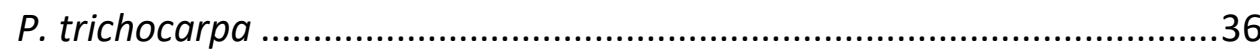

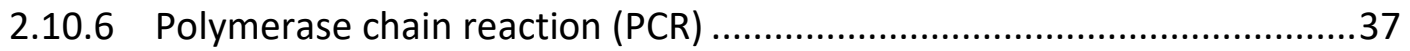

2.10.7 Gel-Electrophoresis (modified from Sambrook and Russell (2001)) ........38

2.10.8 PCR product purification, plasmid extraction and sequencing .................39

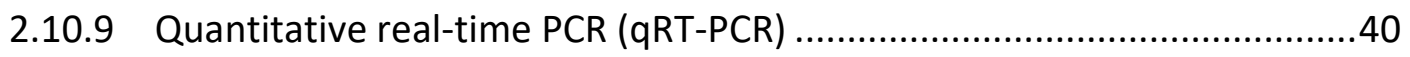

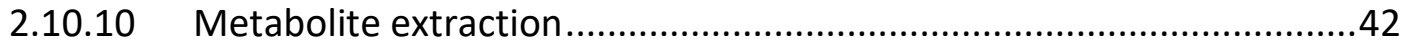

2.10.11 Metabolome analysis with UHPLC-qToF-MS ....................................43

2.10.12 Analysis of metabolome data using MetaboAnalyst 4.0 ….................44

2.11 Test of abscisic acid mutant lines and VALR T-DNA insertion lines .................44

2.11.1 Test of abscisic acid mutant lines............................................................4

2.11.2 Test of VALR T-DNA insertion lines ....................................................45

2.12 Cloning of plasmids and transformation of $A$. thaliana plants .......................45 
2.12.1 Production of electrocompetent Escherichia coli and Agrobacterium tumefaciens cells 45

2.12.2 Transforming electrocompetent cells with plasmid DNA .46

2.12.3 Creating VALR (At4g31330) overexpression lines 48

2.12.4 Targeted deletion of VALR (At4g31330) for loss- or reduction-of-function mutagenesis. .53

2.12.5 Stable transformation of $A$. thaliana using the "Floral dip" method ....... 57

2.12.6 Selection of positively transformed $A$. thaliana plants .......................... 59

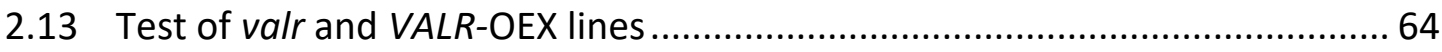

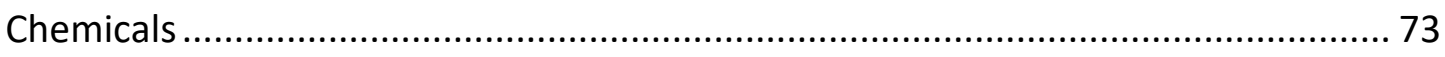

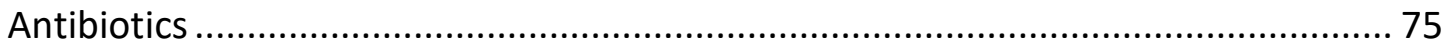

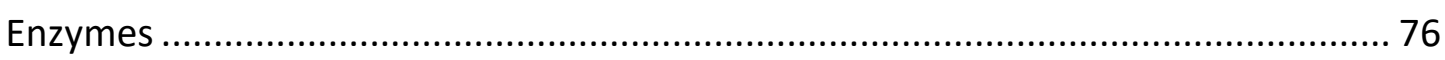

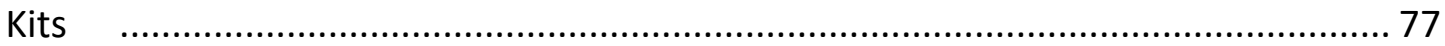

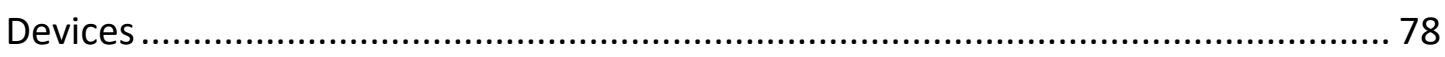

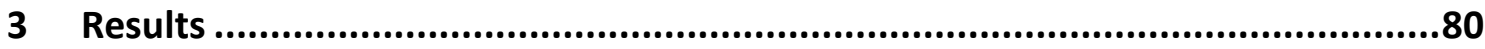

3.1 Impact of experimental conditions on the response to volatiles of L. bicolor in

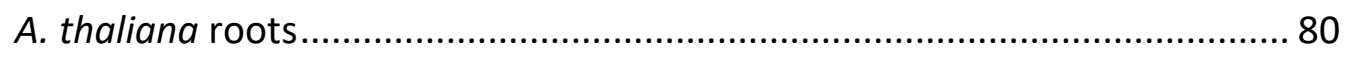

3.1.1 Effect of L. bicolor age on LR formation in A. thaliana ........................... 80

3.1.2 Difference between aluminum trays or cellophane foil for L. bicolor growth on $A$. thaliana development 80

3.1.3 Comparison of $A$. thaliana growth in Phyto agar and Gelrite at different $\mathrm{pH}$ values.

3.1.4 L. bicolor was supported with Farnesyl pyrophosphate to stimulate emission of volatiles and enhance LR formation in A. thaliana 83

3.2 Screens of Arabidopsis mutants potentially involved in volatile-induced lateral root formation

3.2.1 Arabidopsis thaliana cysteine-rich protein kinases (CRKs) homologs in Populus trichocarpa

3.2.2 CRKs involvement in L. bicolor volatiles perception in A. thaliana .85

3.2.3 PER45 and PER59 are not involved in response to volatiles of L. bicolor. 92

3.2.4 Germin-like protein 3 (GLP3) is not involved in response to volatiles of L. bicolor 
3.2.5 Strigolactone are not involved in response to volatiles of L. bicolor ........94

3.3 Testing the suitability of the pGATA23::n/s-GFP-GUS reporter as a marker for volatile-induced LR formation ....

3.4 Determination of the response dose and time of $A$. thaliana roots to volatiles of $L$. bicolor

3.4.1 Response of $A$. thaliana roots is L. bicolor mycelium amount dependent...

3.4.2 Two days of exposure to volatiles of L. bicolor are sufficient to induce enhanced LR formation in A. thaliana 103

3.5 Influence of L. bicolor volatiles on the transcriptome and metabolome of

A. thaliana and P. $x$ canescens. .105

3.5.1 Transcriptional changes A. thaliana roots during exposure to L. bicolor volatiles for one week 105

3.5.2 Transcriptional changes A. thaliana rosettes during exposure to L. bicolor volatiles for one week

3.5.3 Metabolome analysis of $A$. thaliana roots and rosettes

3.5.4 Transcriptional changes in P. $x$ canescens roots exposed to L. bicolor volatiles for different time periods

3.5.5 Transcriptional changes in P. $x$ canescens leaves exposed to L. bicolor volatiles for different time periods

3.5.6 Genes involved in hormone signaling pathways with effects on LR formation were found in transcriptome data

3.6 Screening candidate genes from transcriptome analyses for their involvement in volatile-induced LR formation

3.6.1 ABA is suggested to be involved in volatile-induced $L R$ formation ........131

3.6.2 No VALR (At4g31330) homolog in A. thaliana ......................................133

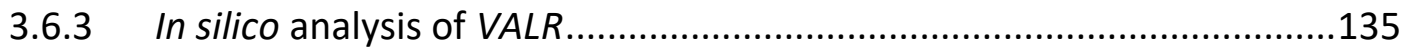

3.6.4 Overexpressing VALR T-DNA insertion lines show less increase in LR density than the wildtype

3.6.5 Creating loss-of function VALR mutants using CRISPR/Cas9 .................138

3.6.6 Inheritance of CRSIPR/Cas9 T-DNA in valr-mutants .............................144

3.6.7 Volatile-induced LR formation in valr-mutants varies ...........................145

3.6.8 Creating overexpression lines of VALR ................................................148 
3.6.9 VALR-overexpression lines did not respond to volatiles 152

3.6.10 GFP expression in VALR overexpression lines .... 154

3.7 Similarity of transcriptome data to published sequencing data..... 155

4 Discussion 158

4.1 Role of CRKs (cysteine-rich receptor-like kinases) in volatile-induced LR formation 158

4.2 Role of peroxidases, germin-like proteins and strigolactones in response to volatiles 160

4.3 Reasons for the waved and aslant root phenotype in all experiments..... 161

4.4 pGATA23::n/s-GFP-GUS marker as a LR formation reporter line 162

4.5 Volatile induced LR formation is dose- and time dependent 164

4.6 L. bicolor volatiles alter gene expression in several processes. 166

4.7 Role of $A B A$ in volatile-induced $L R$ formation 170

4.8 Role of VALR in volatile-induced LR formation

4.9 Overlaps of transcriptome data and published sequencing data 174

5 Conclusion. 176

6 References 179

7 Declaration 199

8 Acknowledgement 200 


\section{List of Abbreviation}

\begin{tabular}{|c|c|}
\hline Abbreviation & Definition \\
\hline ABA & Abscisic acid \\
\hline bp & Base pair \\
\hline Cas & CRISPR-associated \\
\hline CDS & Coding sequence \\
\hline CRISPR & clustered regularly interspaced short palindromic repeats \\
\hline CRK & Cysteine-rich receptor-like protein kinase \\
\hline $\mathrm{dd}_{2} \mathrm{O}$ & double distilled water \\
\hline DNA & deoxyribonucleic acid \\
\hline dNTP & deoxyribose nucleoside triphosphate \\
\hline eGFP & enhanced GFP \\
\hline ET & elongation and tip part of the root \\
\hline FPP & farnesyl pyrophosphate \\
\hline GFP & green fluorescent protein \\
\hline $\mathrm{H}_{2} \mathrm{O}$ & water \\
\hline $\mathrm{H}_{2} \mathrm{O}_{2}$ & hydrogen peroxides \\
\hline HPLC & high performance liquid chromatography \\
\hline INRA & institut national de la recherche agronomique \\
\hline$J A$ & Jasmonic acid \\
\hline LR & lateral root \\
\hline MS & Murashige and Skoog medium \\
\hline NASC & Nottingham Arabidopsis Stock Center \\
\hline $\mathrm{O}_{2}^{-}$ & Superoxide \\
\hline$O_{600}$ & optical density at $600 \mathrm{~nm}$ \\
\hline PCR & Polymerase chain reaction \\
\hline qRT-PCR & quantitative real-time PCR \\
\hline RNA & ribonucleic acid \\
\hline ROS & reactive oxygen species \\
\hline T-DNA & transfer DNA \\
\hline VOC & volatile organic compound \\
\hline wt & wildtype \\
\hline
\end{tabular}




\section{Summary}

Plants, fungi, and prokaryotic organisms emit volatiles for inter-kingdom communication. Some volatiles are emitted in large quantities into the environment and act as climate relevant gases, others are released for pollination or are involved direct or indirect in herbivore defense. Most studies focused on volatiles produced above-ground by plants. Fungi also have complex volatiles patterns, which can impact growth and development of plants effects or mediate communication between plants, bacteria, and fungi. For example, volatiles released by the ectomycorrhizal fungus L. bicolor induce lateral root (LR) formation and root hair growth in the potential host poplar and in the non-host Arabidopsis thaliana.

However, until today it remained unclear, which receptors and signaling pathways in plants are involved in the L. bicolor volatile-induced LR formation.

In this study, we investigated down-stream molecular responses to L. bicolor volatile signaling in A. thaliana and Populus $x$ canescens. To gain insights in the volatile-induced responses, we employed forward and reverse genetic approaches using Arabidopsis mutant lines with roles in hormone signaling and response to volatiles. We exposed mutant lines with potential roles in LR formation associated with ROS homeostasis (per45 and per59), strigolactone biosynthesis and signaling (max2 and max4) and germination (germin-like 3, GLP3) to L. bicolor volatiles and tested the influence on LR density compared to wildtype plants. None of the tested mutant lines showed a different behavior compared to wildtype plants upon volatile exposure. Since cysteine-rich receptor like kinases (CRKs) play roles in stress, growth, and development, we screened a crk knock-out library containing 42 crk lines. We found evidence that CRK4, CRK42 and CRK46 may be involved in volatile perception. However, further analyses of those lines are necessary to strengthen this result.

Furthermore, we investigated the response dose and exposure time to L. bicolor volatiles. By removing the fungus from co-culture with the plant and co-culture the plant with different numbers of fungal plugs we found that co-cultivation with one fungal plug was sufficient to induce enhanced LR formation. With 2, 3, and 4 fungal plugs, the Arabidopsis plants showed similar enhancement of LR densities as those exposed to one plug. This result suggests a repletion of volatile receptors or signaling. 
Furthermore, we showed that a pulse of volatiles exposure of 2 days is sufficient to induced enhanced LR formation.

To identify volatile-induced candidate genes, time-course experiments with increasing duration of volatile exposure were performed and the transcriptomes and metabolomes analyzed. In these studies, we identified a consistently down-regulated gene of unknown function (At4g31330). We further investigated the LR density of At4g31330 T-DNA insertion upon volatile exposure and transformed $A$. thaliana plants with CRIPSR/Cas9 to generate At4g31330 loss-of function mutant lines. Furthermore, we generated At4g31330 overexpression lines and investigated their LR density upon volatile exposure. Compared to wildtype, the At4g31330 CRISPR/lines showed rather low LR density, independent of volatile exposure. The At4g31330 overexpression lines behaved like wildtype upon volatile exposure.

We also detected plant hormone related genes with involvements in stress or LR formation in the transcriptome data. The genes detected by this approach were predominantly related to abscisic acid ( $A B A)$. Thus, we exposed ABA-mutant lines to L. bicolor volatiles and found reduced LR response in aao3-4 and py/8-1 and enhanced response in aba2-1 compared to wildtype, indicating that ABA might be involved in volatile perception or signaling in plants.

Taken together, we showed that LR formation in response to L. bicolor volatiles in planta is a complex process, involving yet unknown proteins, CRKs, and ABA signaling. Since LRs are important for plant water and nutrient uptake, further studies how CRKs and ABA are influenced by fungal volatiles to eventually reprogram root development are needed. 


\section{Introduction}

\subsection{Fungal derived volatiles and their effects on plants}

Most trees are associated with mycorrhizal fungi, which provide nutrients to their host. The mycorrhizal fungi benefit from this interaction by an improved carbon supply (Hobbie, 2006; Söderström et al., 1988). There are different types of mycorrhizal fungi. The most ancient and widespread form are arbuscular mycorrhiza (Finlay, 2008). They are characterized by highly branched hyphae which grow intracellularly without penetrating the host plasma membrane (Finlay, 2008). Another type are ectomycorrhizal fungi. They do not penetrate the host cells but form a network of intercellular hyphae between the epidermal and cortical cells, the so called Hartig net (Finlay, 2008). Besides their role in nutrient supply, ectomycorrhizal fungi also enhance tolerance to abiotic stresses. For example, the ectomycorrhizal fungus Paxillus involutus helps to reduce drought stress in Populus euphratica (Luo et al., 2009b).

Laccaria bicolor, another ectomycorrhizal fungus, increases lateral root (LR) formation in its host Populus $x$ canescens during root-fungus contact, but also when the contact is prevented by a membrane (Felten et al., 2009). This effect is not only observed on symbiotic and colonized host plants but also with the non-host plant Arabidopsis thaliana without direct contact to L. bicolor (Felten et al., 2009). Felten et al. (2009) suggested that L. bicolor increases LR formation in P. canescens by auxin accumulation in the root apex and increase of the apical/lateral auxin transport. Increased LR density was also observed in Cistus incanus and Arabidopsis upon contact-free co-cultivation with the truffles Tuber borchii and Tuber melanosporum (Splivallo et al., 2009). In this case, auxin and ethylene production by the fungi were suggested to be the reason for altered root morphology (Splivallo et al., 2009). However, there was a time difference between the auxin respective fungal induced LR formation and the auxin concentration decreased during ectomycorrhizal root interactions (Luo et al., 2009a; Splivallo et al., 2009). Therefore, it was doubtful whether phytohormones of fungal origin were the initial trigger for increased LR formation. Ditengou et al. (2015) found that volatile organic compounds (VOCs) of L. bicolor induce LR formation in its host $P$. $x$ canescens as well as in the non-host $A$. thaliana (Ditengou et al., 2015). 
VOCs are small molecules that are emitted by plants, fungi, and prokaryotic organisms for inter-kingdom communication (Werner et al., 2016). They are able to move in air and liquids, making them perfect signaling molecules for meditating intercellular and organismal interactions (Effmert et al., 2012). Distinct classes of VOCs are emitted in large quantities into the environment and act as climate relevant gases (Vanzo et al., 2014) or are released for pollination, as direct or indirect defenses or for the establishment of symbiosis (Huang et al., 2019; Kessler and Baldwin, 2001; Wu and Baldwin, 2010). Most studies focused on aboveground VOCs, but in recent years, belowground volatiles emitted by roots, fungi and bacteria got increasing attention (Werner et al., 2016). Belowground VOCs affect growth (Penuelas et al., 2014) and can mediate communication between plants, bacteria and fungi (Falik et al., 2011). Root volatiles also have the ability to alter root chemistry and plant-herbivore interactions of neighboring plants, as recently shown for Centaurea stoebe and Taraxacum officinale (Huang et al., 2019).

VOC patterns of different fungal species have been investigated, e.g. those of Trichoderma ssp., Fusarium oxysporum and Tuber spp. (Bitas et al., 2015; Garnica-Vergara et al., 2016; Guo et al., 2019; Hung et al., 2013; Kottb et al., 2015; Splivallo et al., 2007). There are also studies on the effect of individual VOCs on plants. For example, Lee et al. (2019) investigated the effects of individual VOCs isolated from Trichoderma, on A. thaliana germination and growth (Lee et al., 2019).

VOC patterns from different ectomycorrhizal fungi have also been studied (Müller et al., 2013a). Ditengou et al. (2015) identified sesquiterpenes and among them (-)-thujopsene as an L. bicolor emitted compound that induced LR formation in P. $x$ canescens and A. thaliana. Another sesquiterpene, which is often emitted by fungi and promoting plant growth is $\beta$-caryophyllene (Werner et al., 2016; Yamagiwa et al., 2011). However, when Ditengou et al. (2015) added $\beta$-caryophyllene to $A$. thaliana or $P$. $x$ canescens, the plants did not show a difference in lateral root number compared to non-treated plants, pointing out the specificity of sesquiterpene-mediated signals within and between different species (Ditengou et al., 2015). 


\subsection{VOC-related responses in plants}

Until today, not much is known about the volatile-induced molecular responses in plants that eventually result in increased LR formation. Only a few components involved in LR formation have been identified. Reactive oxygen species (ROS), which mediate developmental reprogramming of roots (Swanson and Gilroy, 2010) and play a major role in triggering and modulating stress responses (Torres, 2010), are involved in the fungal-induced VOC signaling cascade, because Arabidopsis mutants defective in ROS formation did not react to L. bicolor VOCs (Ditengou et al., 2015). Ethylene and auxin, which are involved in root development (Overvoorde et al., 2010; Saini et al., 2013) and play a major role in LR development (Du and Scheres, 2018), were also suggested to induce the VOC-induced signaling cascade (Felten et al., 2009; Splivallo et al., 2009).

For arbuscular fungi, which also emit VOCs to alter root morphology, some insights of the signaling cascade are known. For example, VOCs from the arbuscular mycorrhizal fungus Gigaspora margarita induced LR formation in the host plant Lotus japanicus and the nonhost $A$. thaliana (Sun et al., 2015). Sun and co-workers found that VOCs induced the expression of LjCCD7 (CAROTENOID CLEAVAGE DIOXYGENASE 7) in L. japanicus, which is involved in strigolactone production (Liu et al., 2013). Strigolactones are phytohormones that repress LR formation under optimal plant growth conditions (Kapulnik et al., 2011b; Ruyter-Spira et al., 2011). Under high inorganic phosphate conditions, strigolactone synthesis is suppressed in plants and arbuscular mycorrhiza formation is stopped (Balzergue et al., 2011). These findings indicate that strigolactones might be involved in the signaling pathway during the plant's growth response induced by fungal VOCs (Werner et al., 2016).

VOCs of Trichoderma species affect root architecture as well. 6-pentyl-2H-pyran-2-one (6-PP) was identified in the volatile bouquet from Trichoderma atroviride and was partially involved in enhanced root branching and root hair development in A. thaliana (Garnica-Vergara et al., 2016; Hung et al., 2013). Garnica-Vergara et al. (2016) exposed the Arabidopsis auxin reporter line DR5::GFP to 6-PP and found an enhanced fluorescence signal in the vasculature of the primary root and in LR primordia compared to untreated plants. 
In addition, the expression of the auxin transporters PIN1, 2 and 3 in the primary root was upregulated, while PIN7 was downregulated (Garnica-Vergara et al., 2016). The VOCs of Fusarium oxysporum also affected auxin signaling and transport, because auxin signaling, and transport defective mutants did not show increased LR formation in contrast to the wildtype. Furthermore, the auxin-responsive reporter DR5::GUS was activated in LR primordia of Arabidopsis upon VOC treatment (Bitas et al., 2015).

These results indicate an involvement of auxin signaling and transport in the molecular response to VOCs (Werner et al., 2016). Moreover, they show that VOCs of different fungal species manipulate root development through auxin.

In contrast to other fungal VOCs, roots of $A$. thaliana auxin reporter DR5::GFP plants did not show an increase GFP fluorescence when exposed to L. bicolor VOCs compared to non-treated plants (Ditengou et al., 2015). This result suggests that LR development is not auxin signaling or transport dependent in the interaction of L. bicolor VOCs and Arabidopsis (Ditengou et al., 2015). Therefore, it remains unclear, how VOCs are recognized and which down-stream signaling cascades eventually lead to the morphological changes of the roots.

\subsection{LR formation in A. thaliana roots}

Lateral roots emerge from the primary root of the plant. In dicotyledonous plants they derive from pericycle cells adjacent to the xylem poles, which are called xylem pole pericycle cells (Figure 1; Dubrovsky and Rost, 2012). Some of those cells are primed to become LR primordia, i.e. lateral root founder cells (Figure 1A). Priming occurs in the basal meristem, the zone between the apical meristem and the elongation zone (De Smet et al., 2007). Arabidopsis roots show gravity induced waving when grown vertically on media. LR are developed at the convex side of the primary root, resulting in a left-right alternating pattern due to the waving (Laskowski et al., 2008; Simmons et al., 1995). In Arabidopsis auxin influx carrier AUX1 mutants this pattern is lost, indicating that root waving is AUX1-dependent (De Smet et al., 2007). Furthermore, De Smet et al. (2007) used the auxin-responsive marker DR5::GUS for visualization of the auxin maxima in xylem pole pericycle cells. Besides, a time-course series with the DR5::GUS line and real-time DR5::LUCIFERASE expression revealed periodic pulses in the 
basal meristem (Moreno-Risueno et al., 2010; De Rybel et al., 2010; De Smet et al., 2007), indicating that oscillation of local auxin responses in regular intervals predict LR positions and size of gaps between the LRs (Dubrovsky et al., 2008; Péret et al., 2009). The local accumulation of auxin is achieved by activation of the influx carrier AUX1 in a subset of pericycle cells (Figure 2; Laskowski et al., 2008). However, for LR initiation and LR primordium formation both, auxin influx and efflux mediated by AUX1 and PIN proteins, are necessary (Benková et al., 2003; Casimiro et al., 2001; Marhavý et al., 2013).

Another signal for lateral root founder cell specification might be an auxin-related transient $\mathrm{Ca}^{2+}$ cytosolic increase, because suppressing a $\mathrm{Ca}^{2+}$ increase by pretreatment of the roots with $\mathrm{La}^{3+}$ prevents LR formation in the curved region of the root (Richter et al., 2009). De Rybel et al. (2010) identified GATA23 (GATA TRANSCRIPTION FACTOR 23), a member of the GATA-type transcription factors, as an additional key player during LR founder cell establishment (De Rybel et al., 2010). GATA23 belongs to a group of transcription factors that underlie fluctuating IAA28 (INDOLEACETIC ACID-INDUCED PROTEIN 28)-dependent gene expression along the primary root axis during root development (De Rybel et al., 2010). Because of the tight association between GATA23 and LR formation, GATA23 serves as founder cell identity marker (De Rybel et al., 2010). Its expression in these dedicated pericycle cells is maintained for some time, indicating the position of LR primordia development (De Rybel et al., 2010). GATA23 expression is induced by the AUXIN RESPONSE FACTOR ARF5, 6, 7, 8 and/or 19, whose activation is AUX/IAA28-dependent.

Soon after founder cell specification, primordium formation is initiated in the differentiation zone of roots (Figure 1; Dubrovsky et al., 2011). Founder cells experience anticlinal cell divisions, resulting in stage I primordia (Figure 1B). These cell divisions are followed by further periclinally divisions, leading to a two-layered structure (stage II), a three-layered structure (stage III) and a four-layered structure (stage IV). The following cell division are more complex but result in stage V, VI and VII LR primordia (Figure 1B; Malamy and Benfey, 1997). Further LR growth and elongation is named LR emergence. During the first anticlinal cell divisions, Benková et al. (2003) observed an auxin response maximum in the newly produced cells (Benková et al., 2003b). Auxin induces degradation of SOLITARY ROOT SLR/IAA14, leading to activation of ARF7 and 19, which 
result in nuclear migration in the cells. In the lateral root initiation zone, auxin leads to degradation of SLR/IAA14 and BODENLOS BDL/IAA12 which leads to ARF5,7 and 19 activation (Fukaki et al., 2002; Okushima et al., 2007; De Smet et al., 2010). Activation of ARFs through degradation of IAA leads to the expression of further genes involved in LR induction (reviewed in Fukaki and Tasaka, 2009). Among those are LBD16/ASL18, LBD29/ASL16, LBD18/ASL20, SSL2 and MYB77 (Figure 2) (Fukaki et al., 2006; Goh et al., 2012; Lee et al., 2009; Okushima et al., 2007; Shin et al., 2007).

Taken together, these results show that auxin transport and signaling plays an important and complex role in LR formation. Thus, fungal volatiles might not only affect auxin biosynthesis but also alter auxin transport and signaling, for example by changing local auxin level in specific cell types during LR formation.

\subsection{Influence of abscisic acid, ethylene and jasmonic acid on LR formation}

Many factors such as environment, nutrient and hormones affect LR formation (Fukaki and Tasaka, 2009; Jung and McCouch, 2013). Besides cytokinins, gibberellins and brassinosteroids, abscisic acid (ABA), ethylene, and jasmonic acid (JA) affect LR formation (Figure 2; Fukaki and Tasaka, 2009).

The ABA transcription factor $A B I 3$ (ABA INSENSITIVE 1), which is necessary for ABA signaling, is induced by auxin in LR primordia formation (Brady et al., 2003). When treated with exogenous auxin, the LR responsiveness is reduced in abi3 loss-of function line. In contrast, the era1 (enhanced response to $A B A$ 1) loss-of-function line forms more lateral roots (Brady et al., 2003). ERA1 is a farnesyl transferase, which acts upstream of $A B I 3$ in $A B A$ signaling (Brady et al., 2003). These findings indicate that ERA1 and ABI3-mediated ABA signaling promotes auxin-mediated LR formation (Figure 2; Fukaki and Tasaka, 2009). 


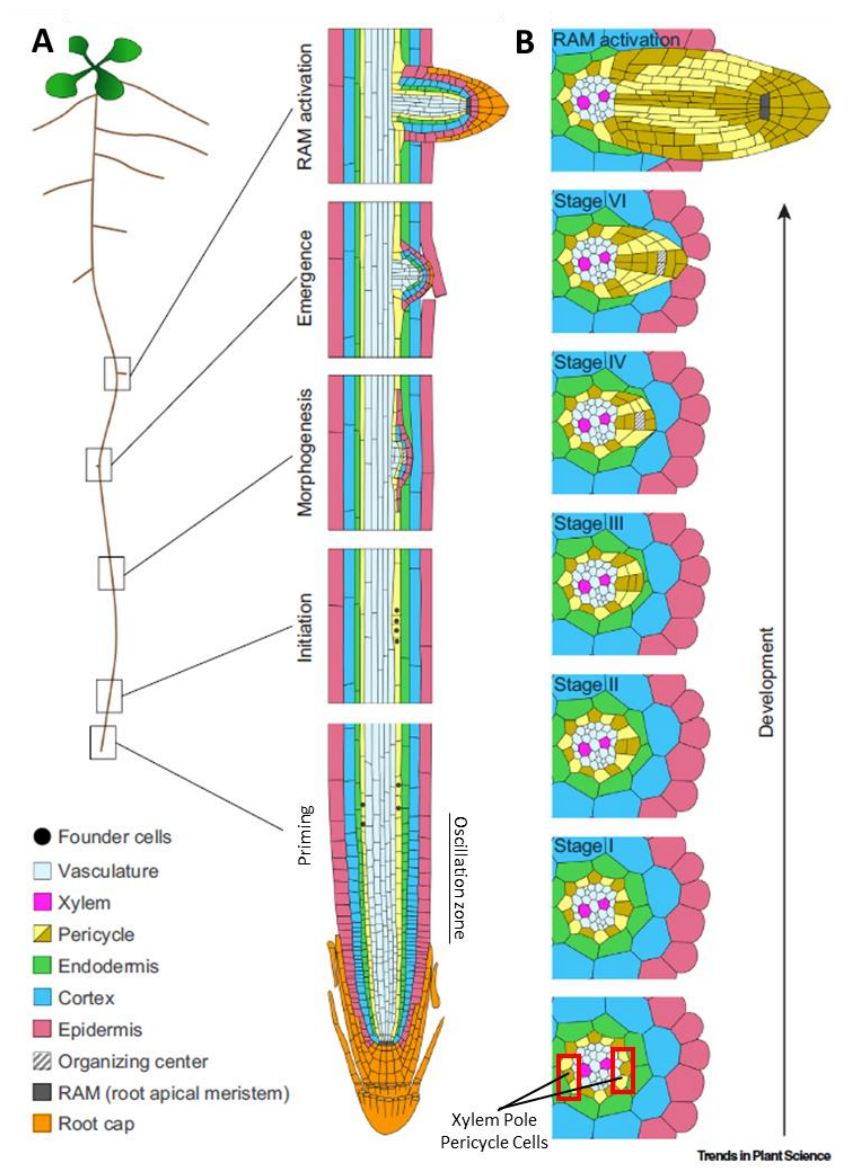

Figure 1. LR development. A) The five main steps of LR formation. The process starts with priming of the xylem pole pericycle cells to become founder cells in the basal meristematic zone. Then, LR founder cells are initiated by nuclear migration of two xylem pole pericycle cells and the first anticlinal division in the differentiation. Periclinally divisions of LR founder cells follow during morphogenesis to form a LR primordium, which grows through the overlying tissues to emerge from the primary root. B) Radial perspective of LR formation. (modified from Banda et al., 2019).

A further plant hormone with an important role in root development is ethylene (Růžička et al., 2007; Street et al., 2015; Swarup et al., 2007). Ethylene negatively influences Arabidopsis LR formation by affecting auxin transport (Figure 2). Negi et al. (2008) showed that exogenous ethylene or its precursor 1-aminocyclopropane-1-carboxylic acid (ACC) reduce LR formation. Furthermore, a mutant that causes overproduction of ethylene, eto1 (ethylene overproducer 1), and the enhanced ethylene signaling mutant, ctr, (CONSTITUTIVE TRIPLE RESPONSE 1), decrease LR formation as well (Negi et al., 2008). 
In consensus with these findings, mutants that block ethylene responses, such as etr1 or ein2 show increased LR formation (Negi et al., 2008).

Decreased LR formation upon ethylene treatment is caused by enhanced PIN3/7 expression (Lewis et al., 2011). PIN3/7 leads to decreased auxin accumulation in the protoxylem pericycle, where LRs are initiated (Lewis et al., 2011).

In contrast to its inhibiting role in LR initiation, ethylene promotes LR emergence of existing LR primordia (Figure 2; Ivanchenko et al., 2008). Interestingly, treatment with low concentrations of ACC (1-aminocyclopropane-1-carboxylic acid), a precursor of ethylene, resulting in increased ethylene synthesis, promote LR initiation as well (Ivanchenko et al., 2008). SIr1 (Solitary Root 1) and arf7 arf19 mutants, which block auxin responses, do not show response to low ethylene levels in LR primordia initiation (Ivanchenko et al., 2008). Mutants, which are defective in ethylene-stimulated auxin biosynthesis, wei2/asa1 and wei7/asb1, decrease the response to higher ethylene levels (Ivanchenko et al., 2008).

In summary, high levels of ethylene inhibit LR initiation, but promote LR emergence and low ethylene levels promote LR initiation through crosstalk with auxin synthesis and signaling (Figure 2).

$\mathrm{JA}$ is commonly known to be involved in wounding and defense responses of plants. However, JA also plays a role in plant growth and development (Figure 2; Turner et al., 2002; Wasternack, 2007). Treatment with exogenous JA inhibits primary root growth and promotes LR formation in Arabidopsis (Cai et al., 2014; Sun et al., 2009; Xue and Zhang, 2007). ASA1 (ANTRANILATE SYNTHASE ALPHA SUBUNIT 1) encodes an enzyme, that mediates JA-induced auxin accumulation and transport (Sun et al., 2009). In contrast to wildtype plants, the jdI1/asa1-1 mutant, which is impaired in JA-induced auxin biosynthesis, shows reduced LR formation upon JA treatment. The JA-mediated auxin accumulation in the basal meristem is also reduced in the jdl1/asa1-1 mutant compared to the wildtype, indicating that JA affects auxin transport in an ASA1-dependent manner (Sun et al., 2009).

Raya-Gonzalez et al. (2012) found that the coil-1 (coronatine insensitive 1) mutant showed uneven LR distribution and insensitivity to JA treatment, indicating that the JA 
receptor COI1 is also involved in the formation and distribution of LRs in Arabidopsis (Raya-González et al., 2012).

Besides the transcription factor ASA1, JA induces the expression of other auxin-related genes as well. Among them are YUCCA2 (Cheng et al., 2006), ASB1/WEI7 (Stepanova et al., 2005) and NIT3 (Kutz et al., 2002), which are involved in auxin-biosynthesis. WEI7 is also involved in ethylene-mediated auxin response (Ivanchenko et al., 2008), indicating an JA-ethylene-auxin crosstalk. Indeed, the ETHYLENE RESPONSE FACTOR 109 (ERF109) expression, which is controlled by ethylene signaling in the vascular tissue (Etchells et al., 2012), is induced upon JA treatment (Cai et al., 2014). Furthermore, ERF109 positively affects LR formation by activating the ASA1 and YUCCA2 expression (Figure 2; Cai et al., 2014). Enhanced expression of ASA1 and YUCCA2 result in increased auxin biosynthesis and accumulation in roots (Cai et al., 2014).

In addition to JA-mediated induction of auxin biosynthesis related genes, JA affects auxin signaling. JA is not able to rescue LR formation in the auxin signaling defective mutants slr1 and arf7 arf19, which do not form LRs under control conditions (Sun et al., 2009). Altogether, these findings suggest that JA-induced LR formation is auxin-dependent (Figure 2; Xu et al., 2020).

\subsection{Further factors that influence LR formation}

ROS might also influence LR formation (Correa-Aragunde et al., 2013; Li and Jia, 2013a; Manzano et al., 2014). Manzano et al. (2014) isolated cells for transcriptomics analysis that were associated with LR primordia development by using the SKP2BBp:GFP marker, which marks cells during founder cell divisions (Manzano et al., 2012). The transcriptome of these cells reveals genes with potential functions in LR development (Manzano et al., 2014). 

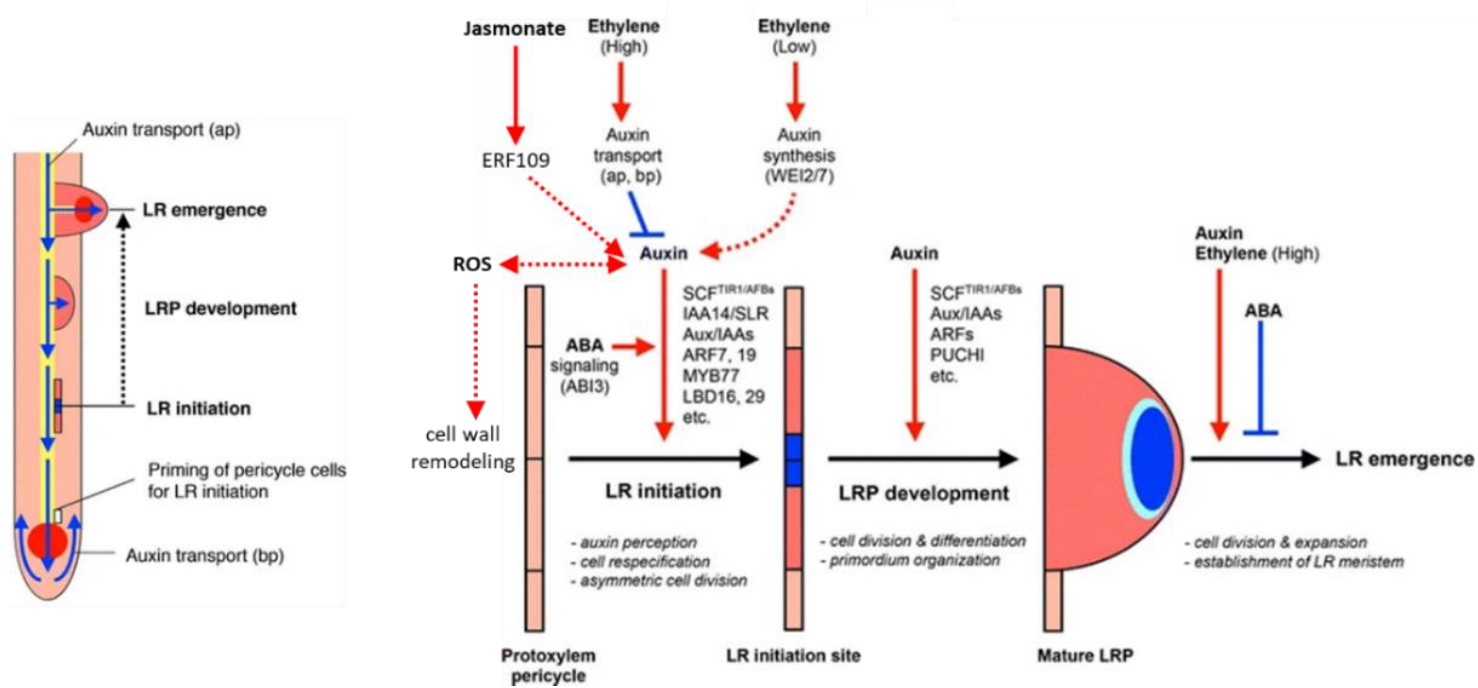

Figure 2. Auxin transport and hormone interaction during LR formation. Polar auxin transport with a balanced influx and efflux in acropetal and basipetal directions is necessary for LR initiation, as well as an auxin gradient during LR primordium formation (blue color). Red arrows indicate positive regulation, dotted arrows indicate indirect regulation. Blue bars indicate negative regulation of $L R$ formation (modified from Fukaki and Tasaka, 2009).

Many of those genes are associated with ROS signaling, for example the transcription factor UPB1, which regulates the expression of several PEROXIDASE (PER) genes (Manzano et al., 2014). PERs modulate the ROS balance in the basal meristem (Tsukagoshi et al., 2010). Further analyses of pUPB1::GFP revealed its expression in early stages of the LR primordium (Manzano et al., 2014). Furthermore, UPB1 overexpression and suppression studies indicated that UPB1-mediated signaling is important for LR primordia development (Manzano et al., 2014). In addition to UPB1, PERs belonging to the class III were highly represented in the transcriptome data of the SKP2BBp:GFP marker line (Manzano et al., 2014). Class III peroxidases are needed for ROS production in the extracellular space (Shapiguzov et al., 2012). Further experiments showed that specific peroxidase activities are necessary for LR emergence but not for LR primordium specification (Manzano et al., 2014).

Further ROS-related genes found in the transcriptome data are members of the cytochrome P450 family, ATRBOHC NADPH oxidase and electron carrier proteins (Manzano et al., 2014). Manzano et al. (2014) suggest, that UPB1 represses PER genes in the transition and elongation zone of the root, leading to increased $\mathrm{H}_{2} \mathrm{O}_{2}$ signaling 
(Manzano et al., 2014). Orman-Ligeza (2016) showed that $\mathrm{H}_{2} \mathrm{O}_{2}$ treatment enhances LR density and reduces primary root length in Arabidopsis seedlings (Orman-Ligeza et al., 2016).

In contrast, loss-off function mutants of the NADPH oxidases AtrbohD and AtrbohF, which produce $\mathrm{H}_{2} \mathrm{O}_{2}$, showed an increased LR number in Arabidopsis seedlings (Ma et al., 2012). Li et al. (2015) showed that LR formation is promoted by localized $\mathrm{O}_{2}{ }^{-}$ accumulation in the primary roots of the AtrbohD and AtrbohF (Li et al., 2015). The $\mathrm{O}_{2}{ }^{-}$accumulation is most likely caused by enhanced peroxidase activity (Li et al., 2015). $\mathrm{Li}$ et al. (2015) suggested that NADPH oxidase may act upstream of peroxidases in Arabidopsis, which affects the localized $\mathrm{O}_{2}{ }^{-}$accumulation and negatively influences $\mathrm{LR}$ development (Li et al., 2015). Taken the described results together, it remains unclear, which kind of ROS affects LR development.

There is also evidence that ROS influence LR development through crosstalk with auxin (Tognetti et al., 2012). For example, $\mathrm{Li}$ and Jia (2013) showed that $\mathrm{H}_{2} \mathrm{O}_{2}$ treatment as well as 8-Br-cGMP (the membrane permeable cGMP analog) treatment increases LR formation in Arabidopsis (Li and Jia, 2013a). 3', 5'-cyclic guanosine monophosphate (cGMP) is a secondary messenger able to regulate polar auxin transport by regulating the PIN family gene expression and promote LR development in Arabidopsis roots (Li and Jia, 2013b). Li and Jia (2013) suggested that $\mathrm{H}_{2} \mathrm{O}_{2}$ acts downstream of cGMP. In Arabidopsis $\mathrm{H}_{2} \mathrm{O}_{2}$ appears to act downstream of auxin to promote $L R$ formation. Ma et al. (2014) suggested that auxin induces haem oxygenase-1 (HO-1, HY1 in Arabidopsis) expression and hydrogen peroxide generation. This ROS production would lead to an expression of cell cycle regulatory related genes, which in turn enhance LR formation (Ma et al., 2014). Furthermore, Orman-Ligeza et al. (2016) showed that ROS promotes cell wall remodeling in a RESPIRATORY BURST OXIDASE HOMOLOGS (RBOH)-mediated manner, thereby facilitating LR growth (Orman-Ligeza et al., 2016). Taken together, these studies clearly demonstrate that ROS can mediate LR formation. Thus, ROS may potentially be involved in volatile-induced LR formation (Figure 2; Ditengou et al., 2015). 
The analyses of ROS related stress responses in CYSTEINE-RICH PROTEIN KINASES (CRKS) T-DNA insertion lines suggest that CRKs play a role in ROS/redox signaling and sensing (Bourdais et al., 2015; Idänheimo et al., 2014; Wrzaczek et al., 2010).

CRKs are a subfamily of receptor like kinases (RLKs) with 44 members in $A$. thaliana. They play roles in different physiological processes and signaling networks in plant development, pathogen defense and abiotic stress responses. CRKs are transmembrane proteins, located in the plasma membrane and characterized by two domains of unknown function 26 (DUF26; PF01657; http://pfam.xfam.org/family/PF01657; stress-antifung domain). Some CRKs, crk28, crk29 and crk42, show slightly longer primary root length than the wildtype (Bourdais et al., 2015), suggesting involvement in root development.

Because of the huge variety of CRKs related responses and especially because of their potential roles in ROS sensing and signaling, abiotic stress responses and root growth, CRKs are potentially involved in volatile-induced LR formation.

\subsection{Aim of the study}

The interaction between tree roots and ectomycorrhizal fungi can affect root architecture and development leading to enhanced LR formation (Barker et al., 1998). This effect has not only been observed in symbiotic interaction of the fungus with its host plant but also in the non-host plant Arabidopsis thaliana in presence of the typical ectomycorrhizal fungus Laccaria bicolor (Felten et al., 2009). Furthermore, LR stimulation occurs even when the plants are separated from the fungus by a membrane (Felten et al., 2009) or without any plant-fungus contact (Ditengou et al., 2015), indicating that LR stimulation is induced in the pre-colonization phase. Phytohormones produced by plants and fungi were discussed to be responsible for this effect (Felten et al., 2009; Splivallo et al., 2009) and Ditengou et al. (2015) showed that VOCs are involved in induction of LR development. However, it is still unclear, which receptors and signaling pathways in planta are involved in the volatile-induced LR formation.

This study focuses on down-stream molecular responses to L. bicolor volatile signaling in the non-host plant $A$. thaliana as well as in the host plant Populus $x$ canescens. The aim was to uncover receptors and/or signaling pathways that are involved in the 
L. bicolor-plant communication. To achieve this goal, forward and reverse genetic approaches using Arabidopsis mutant lines were employed. Arabidopsis mutant lines involved in hormone signaling and VOC-responsive genes were exposed to L. bicolor volatiles to test the influence on LR density. The LR density of mutant lines were compared to wildtype plants to detect potential differences in the volatile-related behavior. Since there is evidence that fungi also emit a range of volatiles besides VOCs (Schnitzler, pers. comm.), I refer here to "volatiles" instead of "VOCs" because attempts to distinguish the biological activities of different classes of fungal-derived volatiles were not made in this work. Furthermore, the response dose and exposure time to volatiles was investigated. By removing the fungus from co-culture with the plant and co-culture the plant with different numbers of fungal plugs, we tested if a pulse of volatiles was sufficient for induction of LR formation.

To identify volatile-induced candidate genes, time-course experiments with increasing duration of volatile exposure were performed and the transcriptomes and metabolomes were analyzed. In these studies, a consistently down-regulated gene of unknown function was identified and further characterized. 


\section{Material and Methods}

\subsection{Cultivation of plants and fungi}

\subsubsection{Growth conditions of Arabidopsis thaliana}

Arabidopsis thaliana seeds Col-0 wildtype and mutant lines were placed on soil (Fruhstorfer Erde Typ N, Hawita Gruppe GmbH, Hirschau, Germany) in $7 \times 7 \times 8 \mathrm{~cm}$ $(270 \mathrm{ml})$ pots (Hermann Meyer KG, Rellingen). Pots with seeds were covered with transparent lids and stratified for 2 days at $4{ }^{\circ} \mathrm{C}$ and $80 \%$ to $90 \%$ humiditiy in darkness. Afterwards, pots were transferred to the climate chamber (AR 75-L, Percival). A. thaliana plants were grown at $16 \mathrm{~h}$ photoperiod (120 to $130 \mu \mathrm{E} \mathrm{m} \mathrm{m}^{-2} \mathrm{~s}^{-1}$; neon tubes (F32T8/TL741, 32 Watt, Philips) and light-emitting diodes (MM21091-LED Classic, 7.4 Watt, Megaman) at $22{ }^{\circ} \mathrm{C}$ and $60 \%$ to $80 \%$ humidity. The transparent lids were removed after 7 to 10 days in climate chamber.

After 6 to 8 weeks, watering stopped for seed maturation. After plants were dry, seeds were collected and stored at room temperature.

\subsubsection{Growth conditions of poplar}

Grey poplar Populus $x$ canescens INRA 717-1B4 (Aiton, France) plantlets were propagated and grown in glass jars $(14 \mathrm{~cm}$ in diameter and $5 \mathrm{~cm}$ in height) with halfstrength MS solidified with Gelrite (Table 1) as described by Müller et al. (2013). Basically, four-week-old poplar plants from a stock culture were propagated under sterile conditions by cutting plants in approximately $1.5 \mathrm{~cm}$ long pieces, each of them containing one petiole. The cuttings were placed vertically in new half-strength MS medium. Jars with 10 to 14 poplar cuttings were either sealed with Leucopore tape (Duchefa Biochemie B.V) for propagation or with Parafilm ${ }^{\circledR} \mathrm{M}$ (Bemis Company Inc.) for co-cultivation experiments. Plants were grown in in the culture room at $23^{\circ} \mathrm{C}$ under long-day conditions (16 h light, $60-85 \mu \mathrm{E} \mathrm{m} \mathrm{m}^{-2} \mathrm{~s}^{-1} / 8 \mathrm{~h}$ darkness) and $30 \%$ to $40 \%$ humidity. 


\subsubsection{Cultivation of Laccaria bicolor}

The ectomycorrhizal fungus Laccaria bicolor S238N-H82 (monokaryon; Maire PD, Orton) was multiplied on modified Pachlewski Medium P5 (Table 1) at $22^{\circ} \mathrm{C}$ in permanent darkness and propagated on new medium every fourth week. For that purpose, the outer, youngest mycelium was cut with a scalpel in $4 \times 4 \mathrm{~mm}$ pieces. Three plugs were transferred to a new petri dish (10 cm diameter) with P5 medium. Fungi for experiments were grown for 2 to 3 weeks.

\subsubsection{Exposure of $A$. thaliana to $L$. bicolor volatiles}

For co-incubation experiments, 4 fungal plugs were either grown on cellophane foil (stripes of $2.5 \times 11 \mathrm{~cm}$ ) which was placed on P20 medium (Table 1;described by Müller et al., 2013) or in self-made-aluminum trays $(2 \times 11 \times 0.5 \mathrm{~cm})$ filled with P20 medium for 11 to 14 days under the described conditions (chapter 2.1.3). Aluminum dishes were folded out of $50 \mu \mathrm{m}$ thick aluminum foil with the help of a wood block $(2 \times 211 \mathrm{~cm})$. Three cellophane foil stripes or aluminum trays were transferred in one squared petri dish $(12 \times 12 \mathrm{~cm})$. For control, aluminum trays with P20 medium or cellophane stripes on P20 medium were incubated under the same conditions (chapter 2.1.3) and for the same time as the fungus.

Table 1. Plant and fungal culture media. Media were prepared with $\mathrm{dd}_{2} \mathrm{O}$ and $\mathrm{pH}$ values were adjusted with a $\mathrm{pH}$-meter ( $\mathrm{pH} 526, \mathrm{WTW} \mathrm{GmbH})$ before adding the agar. Then, the agar was added, and the media were autoclaved $\left(2 \mathrm{bar}\right.$ and $120^{\circ} \mathrm{C}$ and $20 \mathrm{~min}$ (HST 6x6x6, Zirbus technology $\mathrm{GmbH}$ ).

\begin{tabular}{|l|r|l|}
\hline Medium and Compound & \multicolumn{2}{|l|}{ Concentration } \\
\hline Arabidopsis Medium (AM) & 2.20 & $\mathrm{~g} / \mathrm{l}$ \\
\hline MS (Murashige and Skoog) salt (Duchefa Biochemie B.V.) & 10.00 & $\mathrm{~g} / \mathrm{l}$ \\
\hline Sucrose & 1.00 & $\mathrm{~g} / \mathrm{l}$ \\
\hline 2-(N-morpholino) ethanesulfonic acid (MES) sodium salt & 8.00 & $\mathrm{~g} / \mathrm{l}$ \\
\hline Phyto Agar (Duchefa Biochemie B.V.) & & \\
\hline pH 5.8 adjusted with HCl & & \\
\hline
\end{tabular}




\begin{tabular}{|c|c|c|}
\hline $1 / 2$ MS (Murashige \& Skoog) Medium & & \\
\hline \multicolumn{3}{|l|}{ Macro Elements } \\
\hline $\mathrm{NH}_{4} \mathrm{NO}_{3}$ & 1.65 & $\mathrm{~g} / \mathrm{l}$ \\
\hline $\mathrm{KNO}_{3}$ & 1.90 & $\mathrm{~g} / \mathrm{l}$ \\
\hline $\mathrm{CaCl}_{2} \times 2 \mathrm{H}_{2} \mathrm{O}$ & 0.44 & $\mathrm{~g} / \mathrm{l}$ \\
\hline $\mathrm{MgSO}_{4} \times 7 \mathrm{H}_{2} \mathrm{O}$ & 0.37 & $\mathrm{~g} / \mathrm{l}$ \\
\hline $\mathrm{KH}_{2} \mathrm{PO}_{4}$ & 0.17 & $\mathrm{~g} / \mathrm{l}$ \\
\hline \multicolumn{3}{|l|}{ Micro Elements } \\
\hline $\mathrm{H}_{3} \mathrm{BO}_{3}$ & $62 \times 10^{-4}$ & $\mathrm{~g} / \mathrm{l}$ \\
\hline $\mathrm{MnSO}_{4} \times \mathrm{H}_{2} \mathrm{O}$ & 0.01 & $\mathrm{~g} / \mathrm{l}$ \\
\hline $\mathrm{ZnSO}_{4}+7 \mathrm{H}_{2} \mathrm{O}$ & $86 \times 10^{-4}$ & $\mathrm{~g} / \mathrm{l}$ \\
\hline $\mathrm{KI}$ & $83 \times 10^{-5}$ & $\mathrm{~g} / \mathrm{l}$ \\
\hline $\mathrm{Na}_{2} \mathrm{MoO}_{4} \times 2 \mathrm{H}_{2} \mathrm{O}$ & $2.5 \times 10^{-4}$ & $\mathrm{~g} / \mathrm{l}$ \\
\hline $\mathrm{CuSO}_{4} \times 5 \mathrm{H}_{2} \mathrm{O}$ & $2.5 \times 10^{-5}$ & $\mathrm{~g} / \mathrm{l}$ \\
\hline $\mathrm{CoCl}_{2} \times 6 \mathrm{H}_{2} \mathrm{O}$ & $2.5 \times 10^{-5}$ & $\mathrm{~g} / \mathrm{l}$ \\
\hline \multicolumn{3}{|l|}{ Vitamins } \\
\hline Niacin (Merck) & $5 \times 10^{-4}$ & $\mathrm{~g} / \mathrm{l}$ \\
\hline Pyridoxine $\mathrm{HCl}$ (Sigma-Aldrich) & $5 \times 10^{-4}$ & $\mathrm{~g} / \mathrm{l}$ \\
\hline Thiamine $\mathrm{HCl}$ (Merck) & $1 \times 10^{-4}$ & $\mathrm{~g} / \mathrm{l}$ \\
\hline Glycine & 0.002 & $\mathrm{~g} / \mathrm{l}$ \\
\hline Myo-Inositol & 0.100 & $\mathrm{~g} / \mathrm{l}$ \\
\hline Sodium feredetate $\left(\mathrm{C}_{10} \mathrm{H}_{12} \mathrm{FeN}_{2} \mathrm{NaO}_{8}\right)$ & $36.7 \times 10^{-3}$ & $\mathrm{~g} / \mathrm{l}$ \\
\hline Sucrose & 20 & $\mathrm{~g} / \mathrm{l}$ \\
\hline Gelrite & 4 & $\mathrm{~g} / \mathrm{l}$ \\
\hline $\mathrm{pH} 5.8$ adjusted with $\mathrm{KOH}$ & & \\
\hline
\end{tabular}




\begin{tabular}{|l|r|r|l|}
\hline Pachlewski Medium & \multicolumn{1}{|c|}{ P05 } & P20 & \\
\hline Di-ammonium tartrate & 0.50 & 0.50 & $\mathrm{~g} / \mathrm{l}$ \\
\hline Potassium dihydrogen phosphate & 1.00 & 1.00 & $\mathrm{~g} / \mathrm{l}$ \\
\hline Magnesium sulfate $\times \mathrm{HH}_{2} \mathrm{O}$ & 0.50 & 0.50 & $\mathrm{~g} / \mathrm{l}$ \\
\hline Maltose & 5.00 & - & $\mathrm{g} / \mathrm{l}$ \\
\hline Glucose & 20.00 & 1.00 & $\mathrm{~g} / \mathrm{l}$ \\
\hline Gelrite & 20.00 & 12.00 & $\mathrm{~g} / \mathrm{l}$ \\
\hline Kanieltra microelement solution $(1: 10)$ & 1.00 & 1.00 & $\mathrm{ml} / \mathrm{l}$ \\
\hline Thiamine $\mathrm{HCl}(0.1 \%)$ & 0.10 & 0.10 & $\mathrm{ml} / \mathrm{l}$ \\
\hline $\mathrm{pH} 5.8$ adjusted with $\mathrm{KOH}$ & & & \\
\hline
\end{tabular}

Cellophane foil was sterilized by boiling the cut stripes in double distilled water $\left(\mathrm{ddH}_{2} \mathrm{O}\right)$ for $30 \mathrm{~min}$. After exchanging the water, the stripes were boiled again for $30 \mathrm{~min}$. Then, stripes were removed and transferred to round glass petri dishes (diameter $12 \mathrm{~cm}$ ) one by one and pouring $\mathrm{ddH}_{2} \mathrm{O}$ between the layers. Petri dishes were wrapped in aluminum foil and autoclaved twice at 2 bar and $120^{\circ} \mathrm{C}$ and $20 \mathrm{~min}$ (HST 6x6x6, Zirbus technology $\mathrm{GmbH)}$.

For exposure of Arabidopsis thaliana to Laccaria bicolor volatiles without physical contact to the fungus, a bi-compartmented petri dish system described by Ditengou et al. (2015) was used as follows: A line of P20 medium (Table 1) was pipetted from one site of a squared petri dish $(12 \times 12 \mathrm{~cm})$ to the other (approximately 1 to $2 \mathrm{ml}$ medium), separating approximately one fourth of the petri dish (Figure 3). After solidification, a $12 \mathrm{~cm}$ Teflon stripe was added on top of the line to separate the petri dish in two parts (Figure 3). The smaller part (1/4) was filled carefully with P20 medium (25 ml). AM medium (55 $\mathrm{ml}$ ) (Table 1) was poured into the larger part of the petri dish (3/4) after solidification of P20 medium (Figure 3). A. thaliana seeds were surface sterilized by incubating seeds in a mixture of $500 \mu \mathrm{l}$ sodium hypochlorite $(2.8 \%), 500 \mu \mathrm{lddH_{2 }} \mathrm{O}$ and $2 \mu \mathrm{l}$ Triton X-100 for 12 to $15 \mathrm{~min}$ in a $2 \mathrm{ml}$ reaction tube. The hypochlorite mixture was removed with a pipette. Afterwards, seeds were washed with $1 \mathrm{ml}$ sterile $\mathrm{ddH}_{2} \mathrm{O}$ by adding the $\mathrm{dd}_{2} \mathrm{O}$, shake the reaction tube and remove the $\mathrm{ddH}_{2} \mathrm{O}$ with a pipette. This procedure was repeated 5 to 8 times. 
After the last $\mathrm{ddH}_{2} \mathrm{O}$ removal, $0.2 \%$ sterile Agarose (at room temperature, viscose) was added to the seeds and mixed. Seeds were stratified for 2 days at $4{ }^{\circ} \mathrm{C}$ in darkness and transferred on AM medium. Fifteen seeds per petri dish were placed on the upper part (opposite to P20 medium) (Figure 3) and germinated for 4 days at long-day conditions (chapter 2.1.1). Then, L. bicolor fungus was transferred to the P20 medium. Petri dishes were sealed with Parafilm ${ }^{\circledR} \mathrm{M}$ (Bemis Company Inc.) and placed vertically in the growth chamber under long-day conditions (16-hours photoperiod) at $24{ }^{\circ} \mathrm{C}$ during light and $22^{\circ} \mathrm{C}$ during darkness and a humidity of 60 to $80 \%$ (chapter 2.1.1). Co-cultivation occurred for 7 days if not stated different.

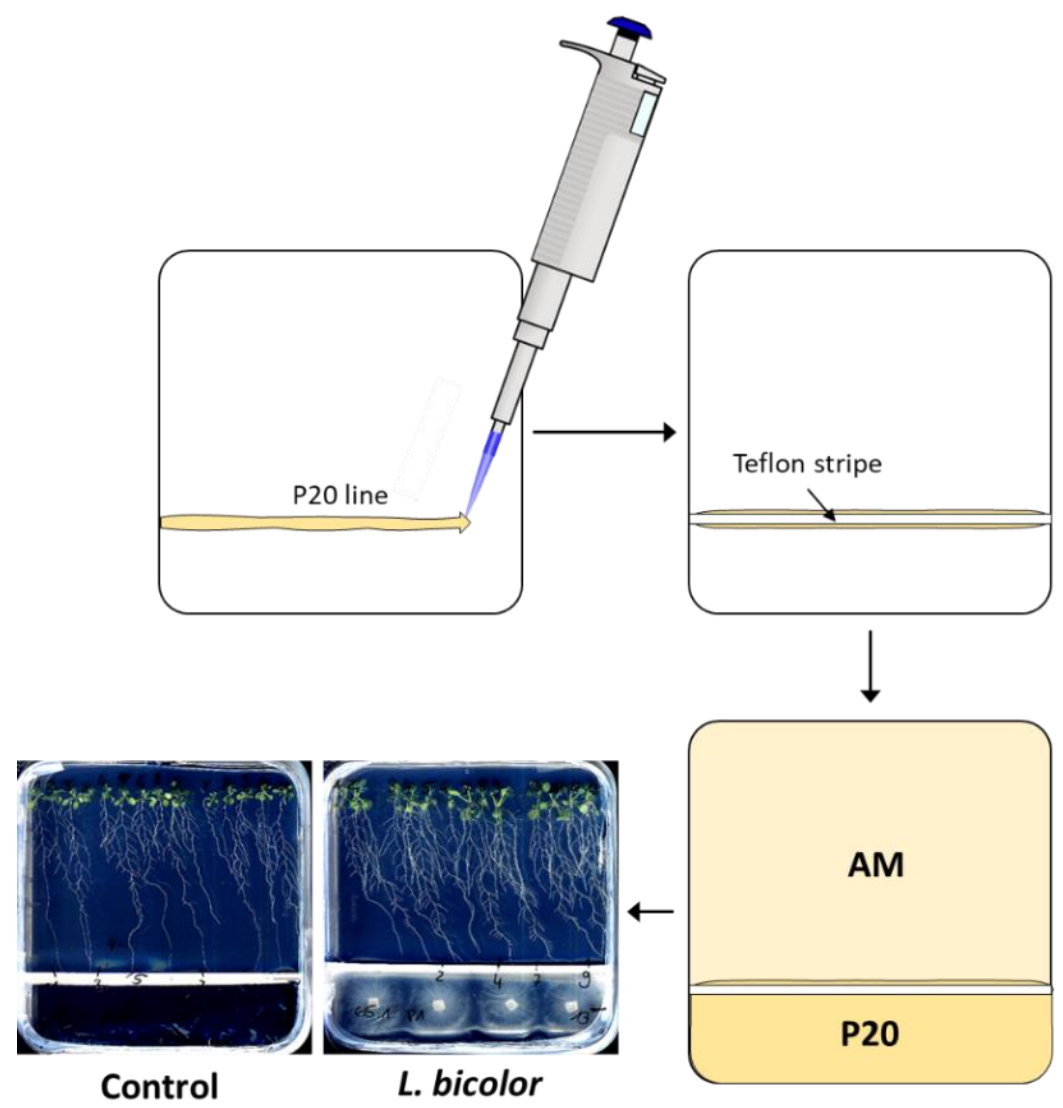

Figure 3. Preparation of bi-compartmented petri dishes. First, a line of P20 was pipetted into the petri-dish. Second, a Teflon stripe was glued in the plate by the P20 line as a barrier between the different media. Then, P20 was poured carefully into the smaller part of the petri dish. After solidification, the A. thaliana medium was added and solidified. A. thaliana seeds were placed on top of the AM medium. L. bicolor, grown on a cellophane membrane, was put on top of the P20 medium. For control conditions, a cellophane membrane without fungus was added. The headspace is achieved by closing the petri dish with its lid. Seedlings on the picture are 11 days old. 


\subsubsection{Exposure of $P . x$ canescens to $L$. bicolor volatiles}

Exposure of P. $x$ canescens plantlets to L. bicolor volatiles was performed similarly as described for A. thaliana (chapter 2.1.4). Instead of AM medium, half-strength MS medium was used. Furthermore, the fungus was added next to the plant instead of below (Figure 6). $1 / 4$ of the medium was removed after solidification under sterile conditions with a scoop to ensure space for the poplar plantlet shoot. For co-incubation, the tops of poplar plantlets were propagated as described (chapter 2.1.2) and grown for 14 days. L. bicolor was grown on cellophane for 12 days.

Co-incubation was performed for max 14 days under long day conditions (chapter 2.1.1) Poplar plants were scanned every harvesting day for determining the primary roots length and be able to calculate the lateral root density (chapter 2.3).

\subsection{Preliminary experiments}

\subsubsection{Influence of fungal age on A. thaliana LR density}

Four plugs of L. bicolor were grown on P20 in aluminum trays either for 9 days or for 18 days. Then, one aluminum dish with fungus or without was added to 4-day-old A. thaliana plants and co-cultivation occurred for 7 days. For each condition, 8 petri dishes with 15 seeds each were prepared. Then, plants were scanned and the LR density was determined quantitatively and compared (chapter 2.3).

\subsubsection{Comparison of aluminum tray and cellophane foil for grown L. bicolor on}

\section{A. thaliana LR formation}

In aluminum trays, L. bicolor was directly grown on the medium. Under these conditions, the mycelium grew on and into the agar. To test the influence of this growth conditions compared to that of aerial mycelium on $A$. thaliana LR formation the fungus was either grown on P20 in aluminum trays or on cellophane foil on P20 for 12 days. A. thaliana seedlings were grown on AM medium for 4 days before start of co-incubation. Then, fungi grown in aluminum trays or cellophane were added and co-incubation lasted for 7 days in the climate chamber under the described conditions (chapter 2.1.4). Plants were scanned and their lateral root growth was examined and compared (chapter 2.3). 


\subsubsection{Comparison of $A$. thaliana growth in Phyto agar and Gelrite at different $\mathrm{pH}$ values}

AM was prepared either with Gelrite $(12 \mathrm{~g} / \mathrm{l})$ or with Phyto-Agar ( $8 \mathrm{~g} / \mathrm{l})$ (Table 1). The pH was adjusted to $4.8,5.3,5.8$ or 6.3 . A. thaliana wildtype seeds were sterilized and stratified as described in chapter 2.1.4. Fifteen seeds were placed on the medium and germinated in the climate chamber for 4 days. Then, L. bicolor grown on P20 for 12 days in aluminum trays was added (Table 1). Co-incubation lasted for 7 days at the described conditions (2.1.4). Afterwards, the plants were analyzed visually and scanned as described in chapter 2.3 .

\subsubsection{Effect of Farnesyl pyrophosphate ammonium on L. bicolor and A. thaliana growth}

Farnesyl pyrophosphate (FPP; Sigma-Aldrich) is a precursor of sesquiterpenes, which are responsible for enhanced lateral root growth (Davis and Croteau, 2000; Ditengou et al., 2015; Sallaud et al., 2009). FPP was diluted with $\mathrm{ddH}_{2} \mathrm{O}$ to final concentrations of $100 \mu \mathrm{M}, 10 \mu \mathrm{M}, 1 \mu \mathrm{M}, 100 \mathrm{nM}, 10 \mathrm{nM}$ and $1 \mathrm{nM}$. Twenty-five $\mu \mathrm{FPP}$ with different concentrations was added to each 12-day-old fungal plug per petri dish at the first day of co-incubation with 4-day-old $A$. thaliana seedlings. Five petri dishes with 15 seeds each were prepared. Co-incubation took place for 7 days. Plants were scanned and their lateral root growth was examined and compared (chapter 2.3).

\subsection{Determination of lateral root density}

In each A. thaliana experiment, 6 to 10 petri dishes with varied number of seedlings per condition were scanned for measuring primary root length, counting the lateral roots, and determining the lateral root density. For scanning, a CanoScan 4400F (Canon Deutschland $\mathrm{GmbH}$ ) and the PhotoStudio (v.5.5, ArcSoft Inc., California, USA) software was used. Figures were taken at $600 \mathrm{dpi}$.

One lateral root was defined as the first order root emerging from the primary root. At one primary root, all lateral roots with a developing stage, that allowed to identify them by eye, were counted. 
For one experiment, $A$. thaliana lateral roots of approximately 50 to 150 seedlings per line and condition were counted either under the stereomicroscope or on scanned photos.

Lateral root of 6 to 8 poplar plantlets per condition and experiment were counted visually every harvesting day.

Primary root lengths for Arabidopsis and poplar plants were measured with the segmented-line tool of ImageJ (Rueden et al., 2017; Schindelin et al., 2012). Once, the distance per pixels were determined by scanning a ruler along with the petri dishes. The scale of 22.7 pixels $/ \mathrm{mm}$ was defined for all experiments.

Arabidopsis lateral roots were counted using the cell counter plugin for Image J (https://imagej.nih.gov/ij/plugins/cell-counter.html).

Lateral root density was determined by dividing the number of lateral roots by the primary root length. If a poplar plantlet developed two or more primary roots, the number of lateral roots and primary root lengths were summed up before calculating the lateral root density. Statistical analyses were conducted in R v3.6.0 (R Core Team, 2019). A linear model or linear mixed model with the repetitions of the experiment as a random factor was fit to the data using the functions ' $1 \mathrm{~m}$ ' from the 'stats' package or 'Imer' from the 'Ime4' package, respectively (Bates et al., 2015; R Core Team, 2019). A one- or two-factorial analysis of variance (ANOVA) was then conducted using the 'Anova' function from the 'car' package' (Fox and Weisberg, 2019). Before performing the ANOVA, data normality and homogeneity of variance was ensured by visual inspection of the residuals of the linear model. Subsequently, a post hoc Tukey's Honest Significant Difference test was performed if the ANOVA showed significant effects, using the 'glht' function from the 'multcomp' package' (Hothorn et al., 2008). $P$ values $\leq 0.05$ indicated significant differences.

\subsection{A. thaliana mutants genotyping}

In order to examine the role of certain A. thaliana genes in volatile perception and signaling, different mutant lines (Table 14) were exposed to L. bicolor volatiles as described in chapter 2.6. The mutant lines were either obtained from the Nottingham Arabidopsis Stock Center (NASC) or provided by other groups as indicated in Table 14. 
The T-DNA insertion lines obtained from NASC were genotyped by PCR. In the valr T-DNA insertion lines, absence of transcript was validated by reverse transcription PCR (RT-PCR) (chapter 2.10.6). For that purpose, gDNA from leaves was extracted like described (chapter 2.10.1). For SALK-, SAIL- or GABI-T-DNA insertion lines, specific primers (SALK-LB, SAIL-LB or GABI_LB) were used, that bind in the T-DNA and amplify into the mutated gene (Table 15). In combination with an appropriate gene primer, this PCR leads to a T-DNA insertion site should not lead to PCR product in homozygous lines due to the large size of the T-DNA construct (see http://signal.salk.edu/cgibin/tdnaexpress?LOCATION=15202752\&CHROMOSOME=chr4\&INTERVAL=5 to identify the location of T-DNA insertion in the gene). All tested plants of per45 and per59 were homozygote (Figure 4). Also, the valr T-DNA insertion lines valr1-5, valr1-10 and valr2 were homozygous for the T-DNA insertion (Figure 4). For valr3 plant 1, 2, and 5 are heterozygous for the T-DNA insertion and plant 3 and 4 are wildtype (Figure 4). Thus, this line was excluded from further analysis.

No PCR product is expected for homozygous lines with gene specific primers for the RT-PCR of the valr T-DNA insertion lines. As control for successful gene amplification, Actin2 was amplified using the primer pair Actin2. Surprisingly, valr1-5 and valr1-10 showed RT-PCR products, although the gDNA was homozygous. These lines were still used in an experiment to test their behavior upon volatile exposure compared to wildtype.

Homozygous T-DNA insertion lines and the respective wildtype ecotype were chosen for further volatile exposure experiments. For experiments, seeds from homozygous plants of the same line were pooled. 
A

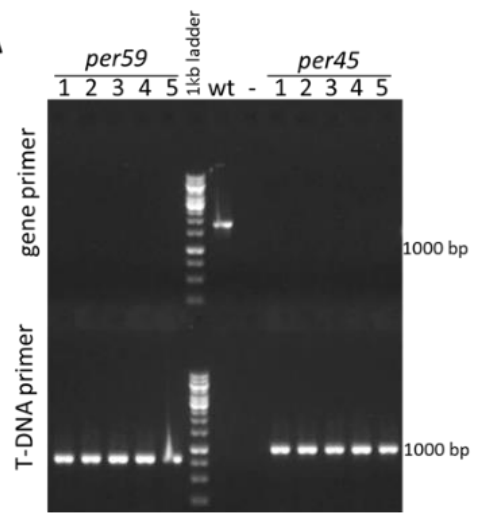

B

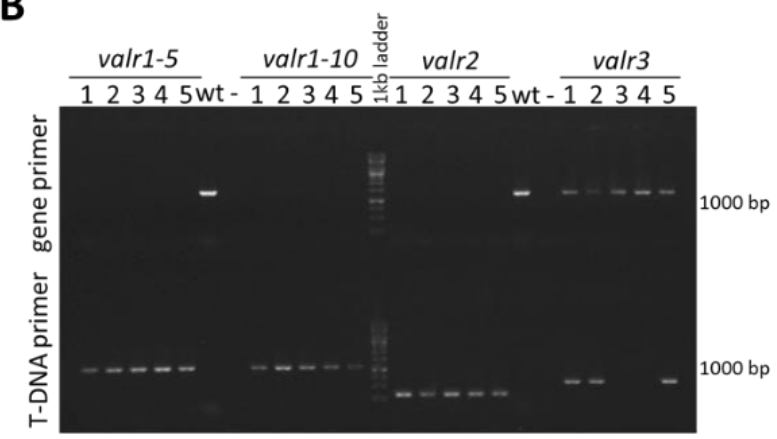

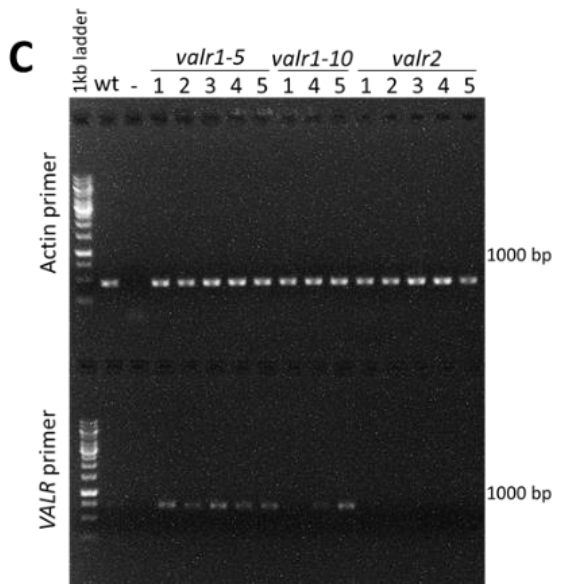

Figure 4. Agarose gels (1.2\%) from genotyping PCRs of T-DNA insertion lines. Gels were run at 110 volts for 15 to $20 \mathrm{~min}$. A) and B) PCR products of leaf gDNA amplified with a gene specific primer pair or one T-DNA specific primer and one gene specific primer (see text for details). A) PCR of T-DNA insertion lines per59 and per45 (lines chosen from microarray data). B) PCR of valr T-DNA insertion lines (lines chosen from transcriptomic data). C) RT-PCR products of leaf cDNA of valr T-DNA insertion lines.

\subsection{Identification of $A$. thaliana cysteine rich receptor-like kinase (CRK) homologues in Populus trichocarpa}

The A. thaliana protein sequences from each CRK described by Bourdais et al. (2015) were searched in the Phytozome 12 database (provided by Joint Genome Institute, JGI; https://phytozome.jgi.doe.gov/pz/portal.html). As reference organism, Arabidopsis thaliana TAIR10 was chosen. Poplar homologues were identified using the BLAST tool at the Phytozome 12 website

(https://phytozome.jgi.doe.gov/pz/portal.html\#!search?show=BLAST). 
The A.thaliana protein sequences were entered one after another and the Populus trichorcarpa v3.0 species was chosen as target species. As target type "Proteome" and as program "BLASTP" was selected. The expected threshold was set to -100. Results were downloaded with comment lines as Excel-files. Duplicates were removed in Excel (Microsoft Office 2010). The resulting table contained 202 P. trichocarpa amino acid sequences with homology to $A$. thaliana CRKs.

Then, the amino acid of each homologue was downloaded from Phytozome 12 and saved as txt-file in FASTA format. CRK amino acid sequences from both plant species were load into Geneious Prime (vR10.0.9, Biomatters, Ltd., Auckland, New Zealand, https://www.geneious.com) as sequence lists, which were aligned using the Multiple Alignment tool (Clustal W Alignment; Cast matrix: BLOSUM; Gap open cost: 10; Gap extended cost: 0.1 ).

All CRKs contain a DUF26 domain (PF01657; Bourdais et al., 2015). This motif was searched in the alignment. Not all poplar homologs had this domain. For further analysis, all homologs without the DUF26 domain were excluded. A phylogenetic tree was created by the Geneious Tree Builder tool with following settings: Genetic Distance Model: Jukes-Cantor; Tree Build Method: Neighbor-Joining; Outgroup: No Outgroup. The resulting tree was crowded. $P$. trichocarpa homologs were filtered according to their e-values from BLASTP. For each CRK, the homologs with the ten-best e-values (from 0 to $4.00 \mathrm{e}-105)$ were considered in the tree.

\subsection{Screens of $A$. thaliana mutants potentially involved in volatile-induced LR formation}

Replicates of all screens are repetitive experiments, which were performed one after another.

\subsubsection{Screen of cysteine-rich receptor like kinases (CRKs) and per45 and per59}

Basically, co-cultivation was performed as described in 2.1.4. Seedlings of $A$. thaliana crk or per45 and per59 mutant lines (Table 14) and wildtype Col-0 seeds were transferred on AM medium in bi-compartmented petri-dishes (chapter 2.1.4). L. bicolor was grown on P20 medium (Table 1) in aluminum trays for 14 days for crk mutant screen and 
11 days for per45 and per59 mutant screen under the described conditions (chapter 2.1.3) and was transferred to the Arabidopsis seeds. Co-cultivation took place for 11 days. Then, plants were scanned and LR density was determined (chapter 2.3).

\subsubsection{Screen of germin-like protein 3 (GLP3) mutants and strigolactone affected mutants}

Basically, co-cultivation was performed as described in chapter 2.1.4. Seedlings of A. thaliana glp3, 35s::GLP3 or max2-1 and max4-1 mutant lines and wildtype Col-0 or Ws-2 were transferred to the AM medium (chapter 2.1.4). L. bicolor was grown on cellophane foil placed on P20 medium (Table 1) for 14 days under the described conditions (chapter 2.1.2). The fungus was directly added to the seeds, without germination. Co-cultivation took place for 11 days. Then, plants were scanned and LR density was determined (chapter 2.3).

\subsection{Analysis of the pGATA23::n/s-GFP-GUS line as a marker for LR development}

pGATA23::nls-GFP-GUS is a marker for lateral root founder cell formation (De Rybel et al., 2010). Seeds of this line were exposed to L. bicolor volatiles as described before (chapter 2.1.4). Seeds were grown for 4 days before co-cultivation with 12-day-old fungus grown on cellophane foil on P20 medium started. Each day of co-cultivation (day $0,1,2,4$ and 7) 5 seedlings per condition were removed from the petri dish and analyzed microscopically (Axioplan Observer.Z1, Carl Zeiss GmbH). For this purpose, roots were separated from shoots using a blade and transferred on an object slide into a drop of tap water, supplemented with a trace of Triton X-100 to reduce surface tension. Roots were covered with cover slides. Primordia, indicated by an accumulation of fluorescent nuclei, were counted as well as lateral roots. For this purpose, the Plan-Apochromat 10x/0.45 M27 objective was used. Pictures were taken with the same objective and the AxioCamMR3 (Carl Zeiss GmbH). The reflector for GFP was 38 Green Fluorescent Protein (Band pass 525/550 nm) and the exposure time $5 \mathrm{~s}$. Brightfield pictures were taken without a reflector at an exposure time of $9 \mathrm{~ms}$. 
In a second experiment, pictures of whole roots were taken using a fluorescence stereomicroscope (Leica Microsystems $\mathrm{GmbH}, \mathrm{M} 205$ FCA) supplemented with a DFC9000 GT camera provided by Leica Microsystems $\mathrm{GmbH}$ and a movable scanning stage. The software Leica Application Suite X (Las X) can merge several pictures from one root to one image. A further advantage was, that roots were examined in situ in the petri dish. Thus, the same root was observed after $0,1,2,4$ and 7 days of fungal co-cultivation. However, the images taken after 7 days were too large in data size to transfer to another computer for analysis. The following conditions were used: 5x magnification; for transmitted light, photos were taken with $18 \mathrm{~ms}$ exposure time and $80 \%$ light intensity; fluorescence photos were taken with 500 ms exposure time at an emission wavelength of $550 \mathrm{~nm}$. For each condition, control, and L. bicolor, eight plants were observed at the indicated exposure days.

\subsection{Determination of volatile response dose and time}

\subsubsection{Determination of volatile response dose}

A. thaliana Col-0 plants were exposed to volatiles different amounts of fungal plugs. Basically, the experiment was performed as described in chapter 2.1.4. Instead of 4 fungal plugs, 1, 2, 3 or 4 plugs were inoculated on cellophane stripes which were placed on P20 medium. The fungus was grown for 14 days (chapter 2.1.3). The Arabidopsis seedlings were transferred to the AM medium (chapter 2.1.4) and grown vertically for 4 days as described (chapter 2.1.42.1.4), before the fungus or the control cellophane foil was added. Co-incubation to place for 7 days. The experiment was performed three times one after another.

\subsubsection{Determination of response time}

A. thaliana Col-0 plants were exposed to volatiles for different time periods. The fungus was grown for 14 days on P20 in aluminum trays. Therefore, it was possible to remove the fungus from the system without any residuals. The Arabidopsis seedlings were transferred to the AM medium (chapter 2.1.4) and grown vertically for 4 days as described (chapter 2.1.4), before the fungus was added. Add day 0 of co-cultivation the 
aluminum trays with the fungus or without as control were added to the Arabidopsis seedlings and incubated under the described conditions (chapter 2.1.4). At day 1, 2, 4 the aluminum trays with the fungus were removed from the plants and the plants were put back in the growth chamber until day 7 after start of co-incubation. At this day, all pants were scanned and LR density was determined as described (chapter 2.3). Hence, all plants had the same age at day of LR density determination. The experiment was performed three times one after another.

\subsection{Harvest plant samples for omics-analysis}

\subsubsection{Harvest of $A$. thaliana plants}

For transcriptomic and metabolomic analysis $A$. thaliana seedlings were exposed to L. bicolor (14-day-old) volatiles for 0, 2, 4 and 7 days before sampling (chapter 2.1.4). The number of petri dishes was adjusted to collect enough plant material for the analyses (Table 2). At each day, harvesting took place after $7 \mathrm{~h}$ of light. Petri dishes were removed quickly from the climate cabinet and opened. Shoots were separated from the roots by cutting them with a blade (Figure 5). For harvesting root parts, the root was cut with a blade as well (Figure 5). Roots, root parts and shoots were collected quickly in separate $2 \mathrm{ml}$ reaction tubes (Eppendorf AG) and frozen in liquid nitrogen. Samples were stored at $-80^{\circ} \mathrm{C}$. Experiments were performed one after another. Each independent experiment is one replicate.

Table 2. Number of petri dishes, each with 15 seeds, for $A$. thaliana harvest. Samples were used for transcriptomic analysis (T) or metabolomic analysis (M). Seedlings were exposed to L. bicolor volatiles (L. bicolor) or kept without fungus (control). On day 0 of harvest, seedlings were 4 days old.

\begin{tabular}{|l|l|c|c|c|c|}
\hline & Day & $\mathbf{0}$ & $\mathbf{2}$ & $\mathbf{4}$ & $\mathbf{7}$ \\
\hline \multirow{2}{*}{$\mathbf{T}$} & Control & 12 & 8 & 2 & 2 \\
\cline { 2 - 6 } & L. bicolor & 12 & 8 & 2 & 2 \\
\hline \multirow{2}{*}{$\mathbf{M}$} & Control & 5 & 5 & 2 & 2 \\
\cline { 2 - 6 } & L. bicolor & 5 & 5 & 2 & 2 \\
\hline
\end{tabular}


A

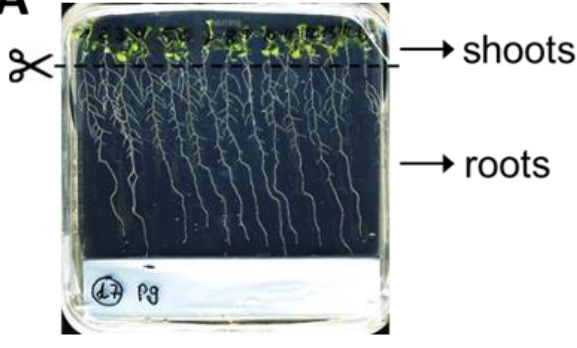

B

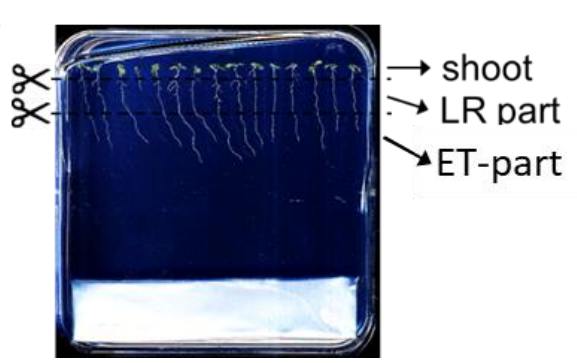

Figure 5. Harvest of $A$. thaliana plants. A) Harvest of whole roots. After volatile exposure, roots were separated from shoots using a blade. B) Harvest of root parts. After two days of volatile exposure, plants were cut in three parts: shoot, lateral root part (LR part) and elongation-tip part. Root parts were transferred to separate tubes, frozen in liquid nitrogen and stored at $-80^{\circ} \mathrm{C}$.

\subsubsection{Harvesting of Populus $x$ canescens plantlets}

P. $x$ canescens plantlets for transcriptomic and metabolomic analysis were exposed to L. bicolor (12-day-old) VOCs for 0, 2, 4, 7 and 14 days. One petri dish contained one plant (Figure 6). The number of plants harvested was adjusted to obtain sufficient material for the analyses (Table 3). The number of plants available varied between repetitions due to irregular growth of poplars. Petri dishes with poplar plantlets were removed from the culture room quickly. After opening, plantlets were separated with a scalpel in 4 pieces: leaves, newly formed stem, remaining part (original cutting) and roots (Figure 6). The parts were collected separately in $2 \mathrm{ml}$ reaction tubes (Eppendorf $\mathrm{AG}$ ) and frozen in liquid nitrogen and stored at $-80^{\circ} \mathrm{C}$. Experiments were performed one after another. Each independent experiment is one replicate.

Table 3. Number of petri dishes each with 1 plantlet for $P$. $x$ canescens harvest. Samples were used for transcriptomic analysis $(T)$ or metabolomic analysis $(M)$. Plantlets were exposed to L. bicolor volatiles (L. bicolor) or kept without fungus (control). On day 0 of harvest, plantlets were 14 days old.

\begin{tabular}{|c|l|c|c|c|c|c|}
\hline & Day & $\mathbf{0}$ & $\mathbf{2}$ & $\mathbf{4}$ & $\mathbf{7}$ & $\mathbf{1 4}$ \\
\hline \multirow{2}{*}{$\mathbf{T}$} & Control & 4 to 5 & 4 to 5 & 4 & 4 & 4 \\
\cline { 2 - 7 } & L. bicolor & 4 to 5 & 4 to 5 & 4 & 4 & 4 \\
\hline \multirow{2}{*}{$\mathbf{M}$} & Control & 2 to 3 & 2 to 3 & 2 to 3 & 2 & 2 \\
\cline { 2 - 7 } & L. bicolor & 2 to 3 & 2 to 3 & 2 to 3 & 2 & 2 \\
\hline
\end{tabular}




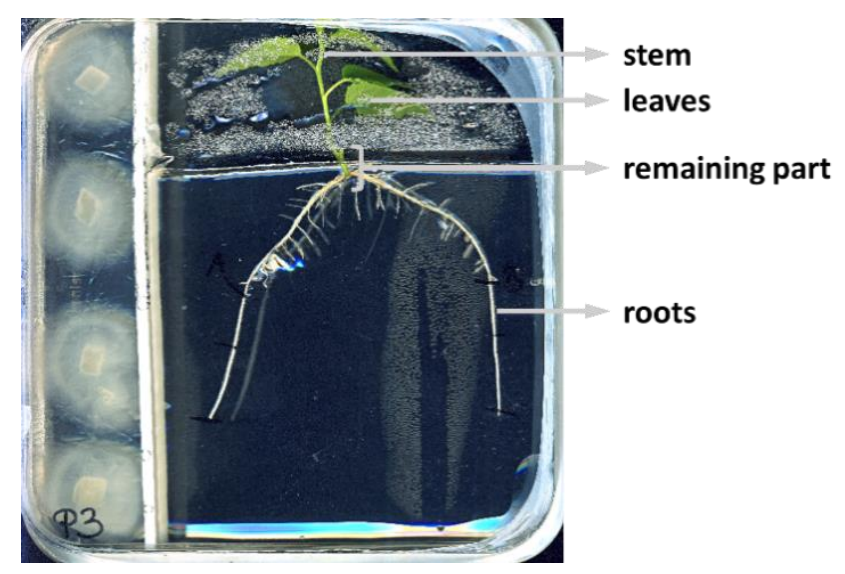

Figure 6. Harvest of poplar plantlets. Plants were cut in 4 pieces: stem, leaves, remaining part, and roots. The parts were collected in different reaction tubes, frozen in liquid nitrogen, and stored at $-80^{\circ} \mathrm{C}$.

\subsection{Molecular biological methods and in silico analysis}

Frozen plant material (50 to $150 \mathrm{mg}$ ) collected in $2 \mathrm{ml}$ reaction tubes supplemented with three stainless steel balls with a diameter of $3 \mathrm{~mm}$ and one ball with a diameter of $4 \mathrm{~mm}$ was removed from $-80^{\circ} \mathrm{C}$ and transferred to liquid nitrogen. The Teflon-adapters (Retsch $\mathrm{GmbH}$ ) used for the Retsch mill (MM400, Retsch $\mathrm{GmbH}$ ) were precooled at $-80{ }^{\circ} \mathrm{C}$ for 30 to $60 \mathrm{~min}$ and transferred to liquid nitrogen as well. The reaction tubes were transferred into the Teflon-adapters under continuous cooling with liquid nitrogen. Then, the adapters were fixed to the mill. Grinding was performed at $30 \mathrm{~Hz}$ for 1:30 min 2 to 3 times. In between, plant material and adapters were cooled in liquid nitrogen. For DNA extractions of $A$. thaliana leaf material, metal balls were replaced by 5-6 disposable glass balls ( $2.5 \mathrm{~mm}$ in diameter).

\subsubsection{DNA extraction of $A$. thaliana leaf samples}

\subsubsection{DNA extraction for genotyping}

For genotyping, DNA was extracted according to a modified protocol from Edwards et al. (1991). Two hundred fifty $\mu$ I Edwards Buffer was added to the grounded plant material (Table 4). The mixture was incubated for $10 \mathrm{~min}$ at $65^{\circ} \mathrm{C}$ and cooled on ice for $10 \mathrm{~min}$. Then, $200 \mu \mathrm{l}$ ice cold chloroform was added to the solution and mixed. 
After centrifugation for $10 \mathrm{~min}$ at $18213 \times \mathrm{g}$ and room temperature, the upper phase was transferred into a new $1.5 \mathrm{ml}$ tube. For DNA precipitation, $300 \mu \mathrm{l}$ ice cold $100 \%$ isopropanol was added, and the sample was incubated on ice for at least $30 \mathrm{~min}$. A 10 min centrifugation step at $18213 \times g$ followed. The supernatant was discarded, and the DNA pellet was washed with $70 \%$ ethanol in $\mathrm{ddH}_{2} \mathrm{O}$. The mixture was centrifuged at $18213 \times \mathrm{g}$ for $10 \mathrm{~min}$. The ethanol was removed with a pipette and the pellet were dried at $37{ }^{\circ} \mathrm{C}$. The pellet was dissolved in $100 \mu \mathrm{l} \mathrm{TE}$ Buffer by incubation at $65{ }^{\circ} \mathrm{C}$ for $10 \mathrm{~min}$ (Table 4). The sample was used for PCR (chapter 2.10.6)

\subsubsection{DNA extraction for sequencing}

To obtain pure genomic DNA (gDNA) for PCR product sequencing, gDNA was extracted using CTAB-buffer (Table 4; adapted from Weigel and Glazebrook (2002)). Milled plant tissue (approximately $100 \mathrm{mg}$ ) was thawed in $600 \mu \mathrm{lCTAB}$-buffer and incubated at $65^{\circ} \mathrm{C}$ for $30 \mathrm{~min}$. Then, $1 \mu \mathrm{l}$ RNase A ( $1 \mathrm{mg} / \mathrm{ml}$; Thermo Fisher Scientific) was added and the sample was incubated at $37^{\circ} \mathrm{C}$ for $15 \mathrm{~min}$ to degrade RNA. In order to dissociate DNA from proteins, $600 \mu \mathrm{l}$ chloroform was added and mixed by inverting the tube. The sample was centrifuged at $1700 \times g$ for $10 \mathrm{~min}$ at room temperature. Four hundred $\mu \mathrm{l}$ of the resulting upper phase was transferred into a new tube and $1 \mathrm{ml}$ ice cold $99 \%$ ethanol in $\mathrm{dd}_{2} \mathrm{O}$ was added. DNA precipitation occurred during centrifugation at $4{ }^{\circ} \mathrm{C}$ at $18213 \times g$ for 30 to $60 \mathrm{~min}$. The supernatant was discarded without dissolving the DNA pellet. Five hundred $\mu \mathrm{l} 70 \%$ ethanol in $\mathrm{ddH}_{2} \mathrm{O}$ was added and the sample was centrifuged at $4{ }^{\circ} \mathrm{C}$ and $18213 \times g$ for $10 \mathrm{~min}$. Ethanol was removed with a pipette and

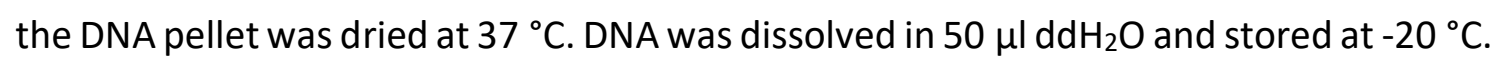

\subsubsection{RNA extraction and cDNA synthesis}

A. thaliana RNA was extracted from approximately $50 \mathrm{mg}$ milled, frozen tissue using either the RNeasy Plant Mini Kit (Qiagen $\mathrm{GmbH}$ ) or with the innuPREP Plant RNA Kit (analytik jena $A G$ ). In both cases, the protocols provided by the manufacturers were followed. RNA extracted with the innuPREP Plant RNA Kit was DNA free. RNA extracted with the RNeasy Plant Mini Kit was cleaned with an on-column DNase digestion using 
the RNase-Free DNase Set (Qiagen $\mathrm{GmbH}$ ). RNA extracted with the innuPREP Plant RNA Kit was already DNA free.

Poplar RNA was extracted according to a modified protocol from Chang et al. (1993). Hundred to $150 \mathrm{mg}$ frozen milled plant material was homogenized in $800 \mu \mathrm{l}$ pre-warmed $65{ }^{\circ} \mathrm{C}$ CTAB-buffer supplemented with $16 \mu \mathrm{l}$ 2-mercaptoethanol to denature RNases (Table 4). The mixture was incubated at $65^{\circ} \mathrm{C}$ on a shaker (Thermomixer, Eppendorf AG) at $1000 \mathrm{rpm}$ for $15 \mathrm{~min}$. Afterwards, the sample was cooled to room temperature and mixed from time to time. Then, $800 \mu$ chloroform-isoamyl alcohol (24:1) was added to dissociate RNA from proteins. The sample was incubated at room temperature while shaking at $1400 \mathrm{rpm}$ for $15 \mathrm{~min}$ in a Thermomixer (Eppendorf AG). After centrifugation at $18213 \times \mathrm{g}$ at room temperature for $5 \mathrm{~min}$., the upper phase containing the RNA was transferred into a new $2 \mathrm{ml}$ reaction tube. Chloroform-isoamyl alcohol was added and the previous washing steps were repeated until no middle phase was visible anymore. After the last washing step, the upper phase was transferred into a new $1.5 \mathrm{ml}$ reaction tube and ice cold $10 \mathrm{mM}$ lithium chloride was added. The volume lithium chloride corresponded to 0.25 -fold of the volume of RNA extract. RNA was precipitated over-night at $4{ }^{\circ} \mathrm{C}$ but not longer than $18 \mathrm{~h}$. Then, the sample was centrifuged at $4{ }^{\circ} \mathrm{C}$ at $18213 \times g$ for $20 \mathrm{~min}$ and $400 \mu \mathrm{l} 65^{\circ} \mathrm{C}$ pre-warmed SSTE buffer was added (Table 4). The solution was mixed for $10 \mathrm{~min}$ at $42^{\circ} \mathrm{C}$ at $850 \mathrm{rpm}$ in a Thermomixer (Eppendorf AG) and $400 \mu \mathrm{l}$ of chloroform-isoamyl alcohol (24:1) was added to the sample. The sample was centrifuged for $5 \mathrm{~min}$ at room temperature at $18213 \times \mathrm{g}$. The upper phase was transferred to a new $1.5 \mathrm{ml}$ reaction tube. The washing steps were repeated until no middle phase was visible. Then, the two-fold volume of $-20^{\circ} \mathrm{C}$ cold $96 \%$ ethanol (in $\mathrm{ddH}_{2} \mathrm{O}$ ) was added to concentrate the RNA. After precipitation at $-20^{\circ} \mathrm{C}$ for $2 \mathrm{~h}$, the sample was centrifuged at $18230 \times g$ at $4{ }^{\circ} \mathrm{C}$ for $20 \mathrm{~min}$. The supernatant was removed, and the RNA pellet was washed with $500 \mu \mathrm{l} 70 \%$ ethanol (in $\mathrm{ddH}_{2} \mathrm{O}$ ). The mixture was centrifuged for $10 \mathrm{~min}$ at room temperature at $18230 \times \mathrm{g}$. A second washing step with $80 \mu \mathrm{l} 70 \%$ ethanol (in $\mathrm{ddH}_{2} \mathrm{O}$ ) followed. The supernatant was discarded, and the RNA pellet was dried in a speedvac (Eppendorf AG) at $45^{\circ} \mathrm{C}$ for 3 to $5 \mathrm{~min}$. The RNA was dissolved in $30 \mu \mathrm{l}$ nuclease-free water (water molecular biology grade, AppliChem $\mathrm{GmbH}$ ) and stored at $-80^{\circ} \mathrm{C}$. RNA concentration was determined spectrophotometrically 
in a NanoDrop 2000 (Thermo Fisher Scientific). As type of measurement "RNA-40" was chosen. As blank, $1 \mu$ l RNase-free water was used. One $\mu$ l of the sample was measured at an absorbance of $260 \mathrm{~nm}, 280 \mathrm{~nm}$ and $230 \mathrm{~nm}$. Pure RNA had a 260/280 ratio of approximately 2.0 and a $260 / 230$ ratio of 1.8 to 2.2 . The concentration was based in the absorbance at $260 \mathrm{~nm}$ and the extinction coefficient of $40 \mathrm{ng}-\mathrm{cm} / \mu \mathrm{l}$.

For cDNA synthesis, the RevertAid First Strand cDNA Synthesis Kit (Thermo Fisher Scientific) was used according to manufacturer's protocol.

\subsubsection{Transcriptome profiling}

One $\mu \mathrm{g}$ of extracted RNA samples (chapter 2.10.2) was sent to Chronix Biomedical (Goettingen, Germany) for RNA sequencing. There, quality control of RNA samples was conducted using the RNA Nano Chip Kit on an Agilent Bioanalyzer 2100. RNA samples with RIN values above 6 were selected for sequencing, if the number of experiments allowed a selection (Table 5). Samples SpK104 and SpP104 were removed from further analysis because the RIN of SpK104 was only 2.5 and 4 replicates remained (Table 5). After enrichment of poly-A RNA using the NEBNext Poly (A) mRNA Magnetic Isolation Module (New England Biolabs) sequencing libraries were prepared using the NEBNext Ultra RNA Library Prep Kit for Illumina (New England Biolabs) according to manufacturer's instructions. Single read sequences of $75 \mathrm{bp}$ were generated using an Illumina NextSeq500 instrument according to manufacturer's instructions.

Table 4. Composition of DNA and RNA extraction buffers. Buffers were prepared with $\mathrm{ddH}_{2} \mathrm{O}$. Stock solutions of Tris- $\mathrm{HCl}$ and EDTA in $\mathrm{ddH}_{2} \mathrm{O}$ were prepared. The $\mathrm{pH}$ of the Tris- $\mathrm{HCl}$ stock solutions was adjusted to the stated values. The SSTE buffer was stored at $-20^{\circ} \mathrm{C}$. Edwards buffer, CTAB buffer and TE buffer was stored at room temperature.

\begin{tabular}{|l|r|}
\hline Buffer and chemicals & Concentration \\
\hline Edwards buffer & $200 \mathrm{mM}$ \\
\hline Tris- $\mathrm{HCl}, \mathrm{pH} 7.5$ & $250 \mathrm{mM}$ \\
\hline $\mathrm{NaCl}$ & $25 \mathrm{mM}$ \\
\hline EDTA (Ethylenediaminetetraacetic acid) & $0.5 \%$ \\
\hline SDS (Sodium dodecyl sulfate) & \\
\hline
\end{tabular}




\begin{tabular}{|l|r|}
\hline & \\
\hline CTAB buffer & $2 \%(\mathrm{w} / \mathrm{v})$ \\
\hline CTAB (Cetyltrimethylammonium bromide) & $100 \mathrm{mM}$ \\
\hline Tris- $\mathrm{HCl}, \mathrm{pH} 8.0$ & $25 \mathrm{mM}$ \\
\hline EDTA (Ethylenediaminetetraacetic acid) & $2 \mathrm{M}$ \\
\hline $\mathrm{NaCl}$ & $2 \%$ \\
\hline PVP (K30; Polyvinylpyrrolidon) & \\
\hline & $0.5 \%$ \\
\hline SSTE buffer & $10 \mathrm{mM}$ \\
\hline SDS & $1 \mathrm{mM}$ \\
\hline Tris- $\mathrm{HCl}, \mathrm{pH} 8.0$ & $1 \mathrm{M}$ \\
\hline EDTA (Ethylenediaminetetraacetic acid) & \\
\hline $\mathrm{NaCl}$ & $10 \mathrm{mM}$ \\
\hline & $1 \mathrm{mM}$ \\
\hline TE buffer & \\
\hline Tris- $\mathrm{HCl}, \mathrm{pH} 8.0$ & \\
\hline EDTA (Ethylenediaminetetraacetic acid) & \\
\hline
\end{tabular}

Sequencing yielded between 4.6 and 19.4 million reads per sample, depending on the type of sample (Table S1). Processing of the raw sequence data (quality control, trimming and filtering) was performed either with fastx-toolkit (http://hannonlab.cshl.edu/fastx toolkit/) for root samples or with fastp (Chen et al., 2018) for rosettes or leaves using the default parameters. After processing, between 4.6 and 19.3 million reads per sample remained (Table S1). The processed sequences were mapped against the transcriptome of either Arabidopsis thaliana (TAIR10, Lamesch et al., 2010) or Populus trichocarpa (Tuskan et al., 2006, version 3.1, downloaded from https://phytozome.jgi.doe.gov/pz/portal.html) using Bowtie 2 (Langmead and Salzberg, 2012). Sixty \% to $97 \%$ of the filtered reads were mapped to a gene model (Table S1).

Bowtie mapping files were summarized to count tables of the gene models in $R$ (R Core Team, 2019). Identification of gene models of expressed transcripts was conducted using the 'DESeq2' package (Love et al., 2014), implemented in R. 
For normalization, the 'DESeqDataSetFromMatrix' function was used. In addition, to investigate separation of groups, principal component analyses were conducted using the 'prcomp' function from the 'stats' package (R Core Team 2019). PCA results were displayed using the 'ggbiplot' function from the 'ggbiplot' package ( $V u, 2011)$.

Data are available on ArrayExpress with the accession numbers E-MTAB-8682 for A. thaliana elongation-tip root and LR root parts in response to L. bicolor volatiles, E-MTAB-8684 for A. thaliana whole roots from time-course experiment and leaves in response to L. bicolor volatiles, E-MTAB-8683 for Populus $x$ canescens roots and leaves in response to $L$. bicolor volatiles (Table S1).

Table 5. RIN values and concentrations of RNA samples send for sequencing. Values were determined using an Agilent Bioanalyzer 2100.

\begin{tabular}{|c|c|c|c|c|c|c|c|}
\hline Plant & Tissue & $\begin{array}{l}\text { Sample } \\
\text { Name }\end{array}$ & $\begin{array}{l}\text { Treat- } \\
\text { ment }\end{array}$ & $\begin{array}{l}\text { Days of } \\
\text { exposure }\end{array}$ & $\begin{array}{l}\text { Experiment } \\
\text { no. }\end{array}$ & RIN & $\begin{array}{l}\text { Concen- } \\
\text { tration } \\
{[\mathrm{ng} / \mu \mathrm{l}]}\end{array}$ \\
\hline \multirow{24}{*}{ 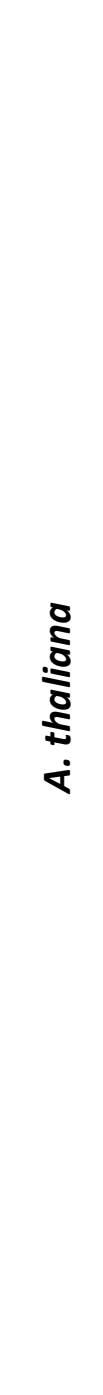 } & \multirow{24}{*}{$\begin{array}{l}\text { ㅁ } \\
\frac{0}{0} \\
\frac{0}{0} \\
\frac{\mathfrak{C}}{3}\end{array}$} & WK70 & C & 0 & 7 & 9.9 & 58 \\
\hline & & WK72 & $C$ & 2 & 7 & 9.7 & 197 \\
\hline & & WK74 & C & 4 & 7 & 9.3 & 746 \\
\hline & & WK77 & C & 7 & 7 & 9.6 & 239 \\
\hline & & WK90 & $C$ & 0 & 9 & 8.3 & 137 \\
\hline & & WK92 & $C$ & 2 & 9 & 8.7 & 447 \\
\hline & & WK94 & C & 4 & 9 & 8.7 & 489 \\
\hline & & WK97 & $C$ & 7 & 9 & 9.5 & 1103 \\
\hline & & WK100 & C & 0 & 10 & 8.4 & 124 \\
\hline & & WK102 & C & 2 & 10 & 9.4 & 209 \\
\hline & & WK104 & $C$ & 4 & 10 & 8.1 & 191 \\
\hline & & WK107 & $C$ & 7 & 10 & 9.7 & 791 \\
\hline & & WP70 & $\mathrm{Lb}$ & 0 & 7 & 9.3 & 81 \\
\hline & & WP72 & $\mathrm{Lb}$ & 2 & 7 & 9.4 & 241 \\
\hline & & WP74 & $\mathrm{Lb}$ & 4 & 7 & 9.1 & 1280 \\
\hline & & WP77 & $\mathrm{Lb}$ & 7 & 7 & 8.7 & 381 \\
\hline & & WP90 & $\mathrm{Lb}$ & 0 & 9 & 9.3 & 61 \\
\hline & & WP92 & Lb & 2 & 9 & 9.1 & 344 \\
\hline & & WP94 & Lb & 4 & 9 & 8.4 & 484 \\
\hline & & WP97 & Lb & 7 & 9 & 9.1 & 1493 \\
\hline & & WP100 & Lb & 0 & 10 & 8.7 & 159 \\
\hline & & WP102 & Lb & 2 & 10 & 8.9 & 315 \\
\hline & & WP104 & $\mathrm{Lb}$ & 4 & 10 & 8.0 & 280 \\
\hline & & WP107 & Lb & 7 & 10 & 8.5 & 1576 \\
\hline
\end{tabular}




\begin{tabular}{|c|c|c|c|c|c|c|c|}
\hline \multirow{18}{*}{ 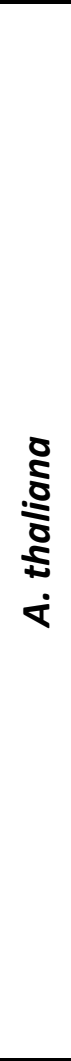 } & \multirow{6}{*}{ 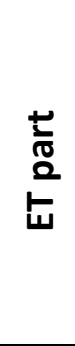 } & 1WSK & C & 2 & 1 & 9.3 & 15 \\
\hline & & 2WSK & C & 2 & 2 & 8.7 & 64 \\
\hline & & 3WSK & C & 2 & 3 & 9.7 & 95 \\
\hline & & 1WSP & $\mathrm{Lb}$ & 2 & 1 & 9.0 & 35 \\
\hline & & $2 W S P$ & $\mathrm{Lb}$ & 2 & 2 & 9.6 & 68 \\
\hline & & 3WSP & Lb & 2 & 3 & 8.6 & 199 \\
\hline & \multirow{6}{*}{ 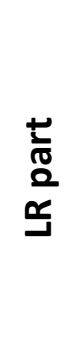 } & 1WRK & $C$ & 2 & 1 & $\mathrm{~N} / \mathrm{A}$ & 7 \\
\hline & & 2 WRK & C & 2 & 2 & 8.8 & 57 \\
\hline & & 3WRK & C & 2 & 3 & 8.9 & 97 \\
\hline & & 1WRP & $\mathrm{Lb}$ & 2 & 1 & 8.7 & 220 \\
\hline & & $2 \mathrm{WRP}$ & Lb & 2 & 2 & 8.7 & 146 \\
\hline & & $3 W R P$ & Lb & 2 & 3 & 8.7 & 183 \\
\hline & \multirow{6}{*}{ 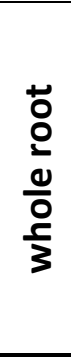 } & $1 \mathrm{WK}$ & C & 2 & 1 & 8.7 & 255 \\
\hline & & $2 \mathrm{WK}$ & C & 2 & 2 & 9.3 & 209 \\
\hline & & $3 W K$ & C & 2 & 3 & 8.4 & 209 \\
\hline & & $1 \mathrm{WP}$ & $\mathrm{Lb}$ & 2 & 1 & 9.3 & 210 \\
\hline & & $2 W P$ & $\mathrm{Lb}$ & 2 & 2 & 9.0 & 210 \\
\hline & & $3 W P$ & Lb & 2 & 3 & 9.0 & 315 \\
\hline \multirow{12}{*}{ 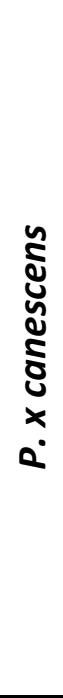 } & \multirow{12}{*}{$\begin{array}{l}\text { 음 } \\
\text { 은 } \\
\frac{0}{0} \\
\frac{0}{3}\end{array}$} & $21 \mathrm{WP}$ & $C$ & 2 & 1 & 6.8 & 108 \\
\hline & & $21 \mathrm{WK}$ & $\mathrm{Lb}$ & 2 & 1 & 4.0 & 114 \\
\hline & & $22 \mathrm{WP}$ & $C$ & 2 & 2 & 6.4 & 514 \\
\hline & & $22 \mathrm{WK}$ & Lb & 2 & 2 & 6.2 & 217 \\
\hline & & 23WP & $C$ & 2 & 3 & 7.1 & 227 \\
\hline & & $23 \mathrm{WK}$ & $\mathrm{Lb}$ & 2 & 3 & 6.4 & 213 \\
\hline & & 71WP & $C$ & 7 & 1 & 6.4 & 121 \\
\hline & & $71 \mathrm{WK}$ & $\mathrm{Lb}$ & 7 & 1 & 6.4 & 62 \\
\hline & & 72WP & $C$ & 7 & 2 & 6.6 & 485 \\
\hline & & $72 \mathrm{WK}$ & $\mathrm{Lb}$ & 7 & 2 & 6.5 & 179 \\
\hline & & 73WP & C & 7 & 3 & 6.3 & 238 \\
\hline & & 73WK & $\mathrm{Lb}$ & 7 & 3 & 6.4 & 114 \\
\hline \multirow{10}{*}{ 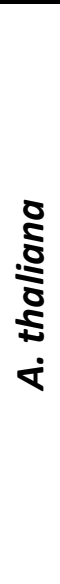 } & \multirow{10}{*}{ 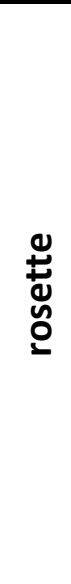 } & SpK54 & C & 4 & 5 & 8.2 & 187 \\
\hline & & SpK74 & C & 4 & 7 & 7.9 & 564 \\
\hline & & SpK84 & C & 4 & 8 & 8.1 & 196 \\
\hline & & SpK94 & C & 4 & 9 & 8.4 & 180 \\
\hline & & SpK104 & $C$ & 4 & 10 & 2.5 & 246 \\
\hline & & SpP54 & Lb & 4 & 5 & 8.4 & 355 \\
\hline & & SpP74 & $\mathrm{Lb}$ & 4 & 7 & 8.0 & 1332 \\
\hline & & SpP84 & Lb & 4 & 8 & 8.4 & 157 \\
\hline & & SpP94 & Lb & 4 & 9 & 7.4 & 536 \\
\hline & & SpP104 & Lb & 4 & 10 & 8.1 & 289 \\
\hline
\end{tabular}




\begin{tabular}{|c|c|c|c|c|c|c|c|}
\hline \multirow{12}{*}{ 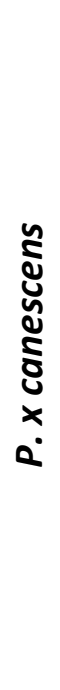 } & \multirow{12}{*}{ 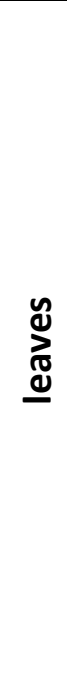 } & 1_K_Blatt_d2 & C & 2 & 1 & 8.6 & 1136 \\
\hline & & 1_Lb_Blatt_d2 & $\mathrm{Lb}$ & 2 & 1 & 8.8 & 1336 \\
\hline & & 1_K_Blatt_d7 & $C$ & 7 & 1 & 8.5 & 1456 \\
\hline & & 1_Lb_Blatt_d7 & $\mathrm{Lb}$ & 7 & 1 & 8.5 & 1480 \\
\hline & & 2_K_Blatt_d2 & $C$ & 2 & 2 & 8.5 & 1284 \\
\hline & & 2_Lb_Blatt_d2 & $\mathrm{Lb}$ & 2 & 2 & 8.7 & 928 \\
\hline & & 2_K_Blatt_d7 & $C$ & 7 & 2 & 8.3 & 1268 \\
\hline & & 2_Lb_Blatt_d7 & $\mathrm{Lb}$ & 7 & 2 & 8.5 & 1212 \\
\hline & & 3_K_Blatt_d2 & $C$ & 2 & 3 & 8.7 & 1100 \\
\hline & & 3_Lb_Blatt_d2 & $\mathrm{Lb}$ & 2 & 3 & 8.8 & 1184 \\
\hline & & 3_K_Blatt_d7 & $C$ & 7 & 3 & 8.3 & 1396 \\
\hline & & 3_Lb_Blatt_d7 & $\mathrm{Lb}$ & 7 & 3 & 8.6 & 836 \\
\hline
\end{tabular}

\subsubsection{Analysis of transcriptome data with MapMan}

For assign AGI and Potri numbers to functional groups, the MapMan software (v. 3.5.1R2; https://mapman.gabipd.org/mapman-download) was used (Thimm et al., 2004; Usadel et al., 2005). The lists of DEGs containing the AGI locus or Potri numbers were load into MapMan along with the corresponding log2-fold changes. For functional group analysis, the lists were mapped to the reference map "Ath_AGI_LOCUS_TAIR10_Aug2012" for Arabidopsis or to "Ptrichocarpa_v3.0_210_peptide" for poplar DEGs. The reference maps were downloaded from the MapMan homepage. $P$ values of functional groups were obtained by Wilcoxon rank sum test and Benjamin Hochberg corrected in the MapMan software. The relative abundance was calculated by dividing the number of found genes through the total number in the data set.

\subsubsection{Identification of VALR homologous genes in A. thaliana and P. trichocarpa}

To investigate whether VALR (At4g31330) has homologous genes in A. thaliana, a BLASTP was performed similar as described in chapter 2.5. For this purpose, the Phytozome 12 website (https://phytozome.jgi.doe.gov/pz/portal.html) was used as well. Potential homologous genes were identified by blasting the amino acid sequence of VALR against the Arabidopsis thaliana TAIR10 or the P. trichocarpa v3.0 reference. The expected threshold was set to -1 . The amino acid sequences of the potential 
homologs were load into Geneious Prime (v. 2020.2.2) and aligned using the Clustal Omega (1.2.2) tool with the default settings. The tree was created with the Geneious Tree Builder as described (chapter 2.5).

\subsubsection{Polymerase chain reaction (PCR)}

The primer for PCR were designed in Geneious Prime (vR10.0.9, Biomatters, Ltd., Auckland, New Zealand, https://www.geneious.com) and obtained from Microsynth Seqlab (Goettingen, Germany) in a dried status. The manufacturer's suggested amount of molecular biology grade water (AppliChem $\mathrm{GmbH}$ ) to obtain a concentration of $100 \mu \mathrm{M}$ was added. The primer were dissolved at room temperature while continuous shaking (Thermomixer, Eppendorf AG).

For PCRs, primers were dissolved with molecular biology grade water (AppliChem $\mathrm{GmbH}$ ) to a concentration of $10 \mu \mathrm{M}$ (Table 15).

For PCRs either Taq-DNA-Polymerase (Thermo Fisher Scientific) or Phusion High Fidelity DNA Polymerase (Thermo Fisher Scientific) were used.

Taq-DNA-Polymerase was used for genotyping and colony-PCRs. PCR components are listed in Table 6. For colony PCRs, a bacterial colony was used as a template. Cycling conditions for Taq-DNA-Polymerase were as follows: initial denaturation at $94{ }^{\circ} \mathrm{C}$ for $5 \mathrm{~min}$, followed by 35 cycles 1 ) denaturation at $94^{\circ} \mathrm{C}$ for $30 \mathrm{~s}, 2$ ) annealing at 56 to $58^{\circ} \mathrm{C}$ depending on the melting temperatures of the primer for $45 \mathrm{sec}$. (Table 15), 3) elongation for $1 \mathrm{~min}$ per $1 \mathrm{~kb}$ at $72^{\circ} \mathrm{C}$. Final elongation occurred for $5 \mathrm{~min}$ at $72{ }^{\circ} \mathrm{C}$. The Phusion DNA Polymerase has a proofreading function and was used for cloning and sequencing. Cycling conditions for Phusion DNA Polymerase were: initial denaturation at $98{ }^{\circ} \mathrm{C}$ for $30 \mathrm{~s}$, followed by 35 cycles 1 ) denaturation for $10 \mathrm{~s}$ at $98^{\circ} \mathrm{C}, 2$ ) annealing for $1 \mathrm{~min}$ at 57 to $59^{\circ} \mathrm{C}$ depending on the melting temperatures of the primer (Table 15), 3) elongation for $30 \mathrm{~s}$ per $1 \mathrm{~kb}$ at $72^{\circ} \mathrm{C}$. Final elongation occurred for $10 \mathrm{~min}$ at $72{ }^{\circ} \mathrm{C}$. All PCR reactions were conducted in a Labcycler (Sensoquest). 


\subsubsection{Gel-Electrophoresis (modified from Sambrook and Russell (2001))}

In order to validate PCR products, gel electrophoresis was performed. For PCR products smaller than $900 \mathrm{bp}$, a $2 \%(\mathrm{w} / \mathrm{v})$ Agarose was prepared and for products larger than $900 \mathrm{bp}$ a $1.2 \%(\mathrm{w} / \mathrm{v})$ Agarose was used. The required amount of Agarose (Biozym Scientific $\mathrm{GmbH}$ ) was dissolved in 1x Tris-acetate-EDTA buffer (diluted with $\mathrm{ddH}_{2} \mathrm{O}$ from 50x Tris-acetate-EDTA buffer) by heating the mixture in a microwave oven (Table 7). Hundred $\mathrm{ml}$ Agarose were supplemented with $3 \mu$ ethidium bromide to stain nucleic acids with a fluorescent tag. The Agarose was poured in a transparent gel tray supplemented with a comb and solidified at room temperature. Electrophoresis was performed in Mini-Sub Cell GT Cell gel chambers (BioRad Laboratories Inc.). As running buffer, 1x Tris-acetate-EDTA buffer was used (Table 7). Electrophoresis was conducted at 100 to 140 Volt for 20 to 30 min. Photos of gels were taken with an Intas Gel IX Imager (Intas) which was supplied with UV-light. The UV-light induces the fluorescent tag, which is bound to the nucleic acids.

Table 6. PCR composition used in in this work. The buffer, the $\mathrm{MgCl}_{2}$ and the nucleasefree water were supplied with the polymerases (Thermo Fisher Scientific). The dNTP mix was mixed out of dATP, dCTP, dGTP and dTTP (10 mM each; Thermo Fisher Scientific).

\begin{tabular}{|l|r|r|}
\hline Composition & Volume & Final concentration \\
\hline Taq DNA Polymerase reaction & $5.0 \mu \mathrm{l}$ & \\
\hline $10 x$ Taq buffer with $\left(\mathrm{NH}_{4}\right)_{2} \mathrm{SO}_{4}$ & $4.0 \mu \mathrm{l}$ & $2.0 \mathrm{mM}$ \\
\hline $\mathrm{MgCl}_{2}(25 \mathrm{mM})$ & $1.0 \mu \mathrm{l}$ & $0.2 \mathrm{mM}$ each \\
\hline dNTP mix $(10 \mathrm{mM}$ each) & $1.0 \mu \mathrm{l}$ & $0.2 \mu \mathrm{M}$ \\
\hline Forward primer $(10 \mu \mathrm{M})$ & $1.0 \mu \mathrm{l}$ & $0.2 \mu \mathrm{M}$ \\
\hline Reverse primer $(10 \mu \mathrm{M})$ & $3.0 \mu \mathrm{l}$ & $10 \mathrm{pg}-1 \mu \mathrm{g}$ \\
\hline Template DNA or & - & $1 \mathrm{U}$ \\
bacterial colony & $0.2 \mu \mathrm{l}$ & \\
\hline Taq DNA Polymerase $(5 \mathrm{U} / \mu \mathrm{l})$ & to $50 \mu \mathrm{l}$ & \\
\hline Water, nuclease free & & \\
\hline \multicolumn{2}{|r|}{} \\
\end{tabular}




\begin{tabular}{|c|c|c|c|}
\hline \multicolumn{2}{|c|}{ Phusion DNA Polymerase reaction } & & \\
\hline \multicolumn{2}{|c|}{ 5x Phusion High Fidelity Buffer } & $10.0 \mu \mathrm{l}$ & \\
\hline \multicolumn{2}{|c|}{ dNTP mix (10 mM each) } & $1.0 \mu \mathrm{l}$ & $0.2 \mathrm{mM}$ \\
\hline \multicolumn{2}{|c|}{ Forward primer $(10 \mu \mathrm{M})$} & $1.5 \mu l$ & $0.3 \mu \mathrm{M}$ \\
\hline \multicolumn{2}{|c|}{ Reverse primer $(10 \mu \mathrm{M})$} & $1.5 \mu \mathrm{l}$ & $0.3 \mu \mathrm{M}$ \\
\hline Template DNA & $\begin{array}{l}\text { gDNA or } \\
\text { plasmid DNA }\end{array}$ & $\begin{array}{l}2.0 \mu \mathrm{l} \\
10 \mathrm{ng}\end{array}$ & \\
\hline \multicolumn{2}{|c|}{ Phusion DNA Polymerase (2 $\mathrm{U} / \mu \mathrm{l})$} & $0.5 \mu \mathrm{l}$ & $0.02 \mathrm{U} / \mu \mathrm{l}$ \\
\hline \multicolumn{2}{|c|}{ Water, nuclease free } & to $50 \mu \mathrm{l}$ & \\
\hline
\end{tabular}

Table 7. Composition of 50x Tris-acetate-EDTA buffer. The buffer was prepared with $\mathrm{ddH}_{2} \mathrm{O}$. EDTA solution was prepared in advance in $\mathrm{ddH}_{2} \mathrm{O}$ and the $\mathrm{pH}$ of the EDTA solution was adjusted with a pH-meter (pH526, WTW GmbH).

\begin{tabular}{|l|r|}
\hline Compound & Amount / Volume \\
\hline Tris-Base & $242.0 \mathrm{~g}$ \\
\hline Acetic acid & $57.1 \mathrm{ml}$ \\
\hline $0.5 \mathrm{M}$ EDTA (Ethylenediaminetetraacetic acid), & $100.0 \mathrm{ml}$ \\
$\mathrm{pH} 8.0$ adjusted with $\mathrm{NaOH}$ & to $1000.0 \mathrm{ml}$ \\
\hline Double distilled water & \\
\hline
\end{tabular}

\subsubsection{PCR product purification, plasmid extraction and sequencing}

If the PCR product was used for sequencing, it was purified using the innuPREP PCRpure Kit (analytik jena AG). The manufacture's protocol was followed with a minor modification: once the PCR product was bound to the column, it was washed with $600 \mu \mathrm{l}$ $80 \%$ ethanol (in $\mathrm{ddH}_{2} \mathrm{O}$ )(see troubleshooting section in provided protocol for details) PCR products were used for cloning (2.12) and genotyping (chapter 2.12.6.).

For plasmid extraction, 5 to $10 \mathrm{ml}$ bacterial over-night culture was used in the innuPREP Plasmid Mini Kit (analytik jena AG) according to the manual.

PCR product or plasmid concentrations were determined spectrophotometrically in a NanoDrop 2000 (Thermo Fisher Scientific). As type of measurement "DNA-50" was 
chosen. As blank, $1 \mu \mathrm{l}$ nuclease free water (Molecular biology grade, AppliChem GmbH) was used. One $\mu$ l of the sample was measured at an absorbance of $260 \mathrm{~nm}, 280 \mathrm{~nm}$ and $230 \mathrm{~nm}$. Pure DNA had a 260/280 ratio of approximately 1.8 and a 260/230 ratio of 1.8 to 2.2. The concentration was based on the absorbance at $260 \mathrm{~nm}$ and the extinction coefficient of $50 \mathrm{ng}-\mathrm{cm} / \mu \mathrm{l}$.

Purified PCR products were mixed with primers (Table 15) according to manufacturer's protocol for premixed barcode economy runs and send for sequencing to Microsynth Seqlab (Goettingen, Germany).

Resulting sequences were analyzed in Geneious Prime (Biomatters, Ltd., Auckland, New Zealand, https://www.geneious.com) to confirm correct cloning or transformation.

\subsubsection{Quantitative real-time PCR (qRT-PCR)}

qRT-PCR was performed to examine VALR (At4g31330) gene expression (adapted from (Sambrook and Russell, 2001). VALR qRT-PCR primer were designed according to the following criteria: the amplicon size was between 70 to $200 \mathrm{bp}$, the melting temperature was close to $60^{\circ} \mathrm{C}$ and the primers were specific for the gene. The latter point was tested by uploading the potential primer sequence to the nucleotide BLAST (National Center for Biotechnology Information, https://blast.ncbi.nlm.nih.gov/Blast.cgi) and blast it against the Arabidopsis thaliana reference (taxid:3702). Target specific hits showed an e-value below 0.05 and an identity of $100 \%$. The e-value of off target hits were greater than 2.0. As reference genes, UBQ10 and UBC9 were chosen (Czechowski et al., 2005). All qRT-PCRs were carried out with a qTower ${ }^{3} \mathrm{G}$ (analytik jena AG) with following conditions: initial denaturation for at $95{ }^{\circ} \mathrm{C}$ for $2 \mathrm{~min}, 45$ cycles of 1 ) denaturation at $95^{\circ} \mathrm{C}$ for $20 \mathrm{~s}, 2$ ) annealing at $58^{\circ} \mathrm{C}$ for $20 \mathrm{~s}$ and 3) elongation at $72{ }^{\circ} \mathrm{C}$ for $20 \mathrm{~s}$. The qRT-PCR ended with a melting curve, starting from $60{ }^{\circ} \mathrm{C}$ to $95^{\circ} \mathrm{C}$ in $15 \mathrm{~s}$ with an increase of $5{ }^{\circ} \mathrm{C}$ per s. For each sample and primer, three technical replicates were prepared. Data analysis was performed with the qPCRsoft-Software v3.1 (analytik jena AG).

In addition to BLAST, specificity of VALR, UBQ10 and UBC9 primer was tested by running a qRT-PCR as described in Table 8. As template, a pool of cDNA from L. bicolor treated and untreated roots or nuclease free water as negative control was used 
(chapter 2.10.2). The melting curves for each amplicon were analyzed with the qPCRsoft software v3.1 (analytik jena AG).

The melting temperature is amplicon specific. At the specific temperature, the double stranded cDNA is separated in two single stranded molecules, releasing a fluorescence signal which is detected by the qRT-PCR machine. This signal of one amplicon results in one unique peak in the melting curve. Thus, if there is only one peak in the melting curve of the respective amplicon, the used primers amplify only one product and are specific. Furthermore, the primer efficiency was determined by using different cDNA concentrations (see below) as template as follows: After cDNA synthesis, cDNA was diluted 1:10 to the working concentration. This concentration was set to 1 in the dilution series. A pool of all cDNA samples was prepared and used in a dilution series of 1, 1:5, 1:25, 1:125 and 1:625. qRT-PCR reactions were prepared with innuMIX qPCR DSGreen Standard master mix (analytik jena AG) as described in Table 8. Primer pair 2 (RT_AtDUF599for_2 and RT_AtDUF599rev_2) showed the best specificity and efficiency and was used for the analysis of VALR expression studies.

In addition, reference primer target stability was checked with the software qbase ${ }^{+}$ (https://www.qbaseplus.com). The primer efficiencies of the VALR, UBQ10 and UBC9 primers were load into the program as well as a table with Ct values from each sample and technical replicate. The software calculated the coefficient of variation (CV), which was 0.097 for UBC9 and 0.086 for UBQ10. The lower the coefficient, the higher the target stability ( $C V<1$ is considered low-variance). Thus, UBC9 and UBQ10 are suitable reference genes.

Normalized gene expression was determined by using the ddCt quantification tool and considering the primer efficiencies (E) according to the Pfaffl method (Pfaffl, 2001). The following formula was used to calculate the normalized expression (NE):

$\mathrm{GOI}=$ Gene of interest (VALR); RefGene = reference gene (UBQ10, UBC9).

$$
N E=\frac{[1+E(G O I)]^{\Delta C t(G O I)}}{[1+E(\text { RefGene })]^{\Delta C t(\text { RefGene })}}
$$

with $\Delta C t(G O I)=C t(G O I$, Calibrator $)-C t(G O I$, Sample $)$ 
and $\quad \Delta C t($ RefGene $)=C t($ RefGene,Calibrator $)-C t($ RefGene, Sample $)$

\subsubsection{Metabolite extraction}

A. thaliana roots or rosettes were transferred into $2 \mathrm{ml}$ reaction tubes and freeze dried. They weight of the samples varied for root from 0.45 to $5.72 \mathrm{mg}$ and for rosettes from 1.51 to $18.92 \mathrm{mg}$. The complete samples was milled using 5 small metal balls per reaction tube. The tube was placed into a grinding jar and fixed in the mill (MM400, Retsch $\mathrm{GmbH}$ ), which was pre-cooled with liquid nitrogen.

Table 8. A qRT-PCR standard reaction mix according to the innuMIX qPCR DSGreen Standard (analytik jena AG) protocol.

\begin{tabular}{|l|r|r|}
\hline Compound & Volume & $\begin{array}{l}\text { Final } \\
\text { concentration }\end{array}$ \\
\hline 2x innuMIX qPCR DSGreen Standard & $10.0 \mu \mathrm{l}$ & $1 \mathrm{x}$ \\
\hline Forward Primer $(10 \mu \mathrm{M})$ & $0.5 \mu \mathrm{l}$ & $0.25 \mu \mathrm{M}$ \\
\hline Reverse Primer $(10 \mu \mathrm{M})$ & $0.5 \mu \mathrm{l}$ & $0.25 \mu \mathrm{M}$ \\
\hline Template cDNA (1:10) & $2 \mu \mathrm{l}$ & $70.0-100.0 \mathrm{ng} / \mu \mathrm{l}$ \\
\hline $\begin{array}{l}\text { molecular biology grade water } \\
\text { (Applichem GmbH) to } 20 \mu \mathrm{l}\end{array}$ & \\
\hline
\end{tabular}

Rosette material was grounded for 3 min with a vibration frequency of $25 \mathrm{~Hz}$. Roots were grounded two times in the same way as the shoots.

After milling, the sample tube was immediately placed in ice. Two hundred $\mu l$ of extraction solvent (Methanol:Isopropanol: $\mathrm{H}_{2} \mathrm{O}[1: 1: 1] ;-20^{\circ} \mathrm{C}$ ) was added to root material to achieve a final concentration of $5 \mathrm{mg} \mathrm{ml}^{-1}$. For rosettes $500 \mu$ l extraction solvent was used to achieve a concentration of $10 \mathrm{mg} \mathrm{ml}^{-1}$. The extraction solvent was spiked with a final concentration of $0.5 \mu \mathrm{g} \mathrm{ml}^{-1}$ camphor-sulfonic acid and $n$-nitrophenol sodium as internal standards. After mixing, the sample was sonicated for $15 \mathrm{~min}$ sonication on ice in an ultrasonic bath. Subsequently, all metal bullets were removed, and the sample was centrifuged at $13000 \mathrm{rpm}$ (Centrifuge $5415 \mathrm{R}$, Eppendorf AG) and 
$4{ }^{\circ} \mathrm{C}$ for $10 \mathrm{~min}$. The supernatant of the sample was transferred to a glass vial (with integrated insert of $200 \mu \mathrm{l}$ ) and stored for analysis at $-80^{\circ} \mathrm{C}$.

\subsubsection{Metabolome analysis with UHPLC-qToF-MS}

The following solvents were used as mobile phase: solvent $A: 0.1 \%$ formic acid in $\mathrm{H}_{2} \mathrm{O}$, solvent B: $0.1 \%$ formic acid in acetonitrile.

Before measuring the samples, the system was purged (increasing from $0 \%$ solvent $B$ to $100 \%$ solvent B; both steps for 2-3 min) and the column equilibrated (decreasing from $95 \%$ solvent $B$ to $50 \%$ solvent $B$ and $5 \%$ solvent B; each step for $20-30$ min at $400 \mu\left(\mathrm{min}^{-1}\right)$. The calibration of the mass spectrometer was performed two times by using the Na-formiat cluster $(50 \mathrm{ml} \mathrm{H} 2 \mathrm{O}, 50 \mathrm{ml}$ Isopropanol, $1 \mathrm{ml}$ sodium hydroxide (1 M) and $0.2 \mathrm{ml}$ formic acid).

For each sample, two technical replicates were measured in both the positive and the negative ionisation mode of the electrospray ionization source. Table 9 shows the applied chromatographic method.

Data acquisition was performed with the HyStar v3.2 software (Bruker Daltonic). Processing of the acquired data were realized by Expressionist for MS 10.5 software (Genedata, Basel, Switzerland) following a 4-step workflow: the first step was the reduction of chemical noise in the data set. In step 2 the retention times were aligned. Step three of data processing identified $\mathrm{m} / \mathrm{z}$ features of the acquired data by using the summed-peak-detection feature implemented in the Expressionist for MS software. The fourth step was used for isotope (13C) clustering of peaks that were present in at least $10 \%$ of mass spectra. Clusters with just one member (singletons) were removed from further analysis.

Features, which where "filtered" by this procedure were annotated to possible metabolites by using an inhouse version of the MassTRIX metabolite annotation server (Suhre and Schmitt-Kopplin, 2008; Wägele et al., 2012; Witting et al., 2014).

Features obtained after processing were analyzed in R (R Core Team, 2019). T-tests with adjusted p-values (False Discovery Rate method) were used to identify significant regulated metabolites, using the 't.test' and the 'p.adjust' functions from the 'stats' package (R Core Team, 2019). Principal component analysis was performed using the 
'prcomp' function from the 'stats' package (R Core Team 2019). PCA results were displayed using the 'ggbiplot' function from the 'ggbiplot' package (Vu, 2011).

Table 9. Chromatographic method parameters.

\begin{tabular}{|l|l|}
\hline Parameter & Condition \\
\hline Injection volume & $5 \mu \mathrm{l}$ \\
\hline Flow rate & $400 \mathrm{ml} / \mathrm{min}$ \\
\hline Gradient of mobile phases & $0-1 \mathrm{~min} 5 \%$ solvent B \\
& $1-15$ min $5 \%-40 \%$ solvent B \\
& $15-18$ min $40 \%-70 \%$ solvent B \\
& $18-23$ mins $70 \%-5 \%$ solvent B \\
\hline Oven & $40^{\circ} \mathrm{C}$ \\
\hline
\end{tabular}

\subsubsection{Analysis of metabolome data using MetaboAnalyst 4.0}

For pathway grouping of predicted metabolites, the annotated HMDB ID list from significantly intensified potential metabolites was load into the Pathway Analysis tool of MetaboAnalyst 4.0

(https://www.metaboanalyst.ca/MetaboAnalyst/ModuleView.xhtml; Chong et al., 2019).

All compounds that were matched to the database were considered for further analysis. The Hypergeometric test was selected for over-representation analysis and relative betweenness centrality was selected for node-importance measure for topological analysis. As a pathway library, Arabidopsis thaliana (thale cress) (KEGG) was chosen. Matches and $p$ values were calculated in MetaboAnalyst.

\subsection{Test of abscisic acid mutant lines and VALR T-DNA insertion lines}

\subsubsection{Test of abscisic acid mutant lines}

Homozygous mutant lines were identified as described in chapter 2.4. A. thaliana mutant lines and wildtype Col-0 were germinated for 4 days under the described conditions (chapter 2.1.4). L. bicolor was grown for 14 days on cellophane foil, which 
was placed on P20 medium (chapter 2.1.3, 2.1.4). At start of co-cultivation, the fungus or the control cellophane stripes were added to the seedlings as described (chapter 2.1.4) and incubated for 7 days.

\subsubsection{Test of VALR T-DNA insertion lines}

Homozygous mutant lines were identified as described in chapter 2.4. A. thaliana mutant lines and wildtype Col-0 seeds were placed on AM medium in bi-compartmented petri dishes as described in chapter 2.1.4. L. bicolor was grown for 14 days on cellophane foil, which was placed on P20 medium (chapter 2.1.3, 2.1.4). At start of the experiment, the fungus or the control cellophane stripes were added to the seeds as described (chapter 2.1.4) and incubated for 11 days.

\subsection{Cloning of plasmids and transformation of $A$. thaliana plants}

\subsubsection{Production of electrocompetent Escherichia coli and} Agrobacterium tumefaciens cells

E. coli $\mathrm{DH} 5 \alpha$ was used to amplify plasmids. It was grown LB medium (Lysogene Broth) without antibiotics at $37^{\circ} \mathrm{C}$ in darkness (Table 10, Bertani, 1951) in an orbital shaking incubator (Sanyo Gallenkamp PLC).

For generation of electro competent E. coli cells, a pre-culture of E. coli was prepared. For this purpose, $5 \mathrm{ml}$ liquid LB medium were inoculated with $E$. coli and incubated over-night at $37^{\circ} \mathrm{C}$ with constant shaking at $120 \mathrm{rpm}$ in the shaking incubator (Sanyo Gallenkamp PLC). Two $\mathrm{ml}$ of the E. coli culture were used the next day to inoculate two times $500 \mathrm{ml}$ liquid LB separated in two 2 I baffled flasks. E. coli were grown like before under constant shaking of $160 \mathrm{rpm}$ in the shaking incubator (Sanyo Gallenkamp PLC) until an $\mathrm{OD}_{600}$ of 0.5 - 0.8 was achieved. One $\mathrm{ml}$ of the culture was transferred to a $1 \mathrm{ml}$ cuvette (Sarstedt AK \& Co.KG) under sterile conditions and the $\mathrm{OD}_{600}$ was measured using a photometer (Biophotometer 6131, Eppendorf AG). Then, the solution was transferred into sterile and precooled $250-\mathrm{ml}$ centrifugation bottles (Sigma-Aldrich) and chilled on ice for $30 \mathrm{~min}$. After centrifugation at $4{ }^{\circ} \mathrm{C}$ at $5000 \times \mathrm{g}$ for 15 min (J2-HS, Beckman Coulter), the supernatant was discarded and the E. coli cell pellet was re-suspended in $250 \mathrm{ml}$ sterile, ice-cold $\mathrm{ddH}_{2} \mathrm{O}$ and centrifuged as before. This washing step was repeated once. 
Then, the $E$. coli pellet was re-suspended in $125 \mathrm{ml}$ sterile, ice-cooled $\mathrm{ddd}_{2} \mathrm{O}$. After centrifugation, the supernatant was discarded, and the $E$. coli pellet was re-suspended in $5 \mathrm{ml}$ sterile and ice-cold $10 \%$ glycerol. The solution was transferred to a $15 \mathrm{ml}$ reaction tube ("Schraubröhre", Sarstedt AG \& Co. KG) and centrifuged at $4{ }^{\circ} \mathrm{C}$ at $5000 \times \mathrm{g}$ for $15 \mathrm{~min}$. Then, the supernatant was discarded, and the bacterial pellet was resuspended in 0.7 to $1 \mathrm{ml}$ sterile, ice-cold $10 \%$ glycerol. Then, the $E$. coli suspension was aliquoted in $50 \mu \mathrm{l}$ portions in sterile $1.5 \mathrm{ml}$ reaction tubes. E. coli electrocompetent cells were frozen in liquid nitrogen and stored at $-80^{\circ} \mathrm{C}$.

A. tumefaciens strain GV3101-pMP90 was used to transfer the expression clone as T-DNA into $A$. thaliana plants. It was grown with YEB-medium (Table 10) at $28^{\circ} \mathrm{C}$ in darkness in an orbital shaking incubator (Sanyo Gallenkamp PLC). The A. tumefaciens strain is resistant to Rifampicin and Gentamycin and therefore, the YEB-medium was always supplemented with these antibiotics (Rifampicin, $50 \mu \mathrm{g} \mathrm{ml}^{-1}$; Gentamycin, $\left.25 \mu \mathrm{g} \mathrm{ml}^{-1}\right)$.

Electrocompetent $A$. tumefaciens cells were prepared as described before for $E$. coli, but A. tumefaciens was grown in YEB medium supplemented with Rifampicin and Gentamycin at $28^{\circ} \mathrm{C}$.

\subsubsection{Transforming electrocompetent cells with plasmid DNA}

In order to transform E. coli, the electroporation method was used. This method is known to be more efficient when transforming large plasmids compared to the chemical transformation procedure (O'Callaghan and Charbit, 1990). Electro competent E. coli cells were prepared as described in chapter 2.12.1. The E. coli glycerol suspension was thawed on ice. Fifty to 100 ng plasmid DNA (chapter 2.12.3 and 2.12.3) were added to the cells in a small volume (about 1 to $3 \mu \mathrm{l}$ ). After 30 min incubation on ice, the $E$. coli cells were transferred to an ice-cold electroporation cuvette with a $1 \mathrm{~mm}$ gap. Electroporation was performed with the MicroPulse Electroporator (BioRad Laboratories Inc). E. coli cells were electroporated at $1.8 \mathrm{kV}$ for $5.0 \mathrm{~ms}$. Immediately after the pulse, $1 \mathrm{ml}$ LB medium with room temperature was added. 
Table 10. Bacterial growth media. E. coli was grown with LB and A. tumefaciens with YEB. Media were prepared with $\mathrm{ddH}_{2} \mathrm{O}$. Before the agar was added, the $\mathrm{pH}$ was adjusted with a $\mathrm{pH}$ meter (pH526, WTW). The media were autoclaved $\left(2\right.$ bar, $120^{\circ} \mathrm{C}, 20 \mathrm{~min}$ (HST 6x6x6, Zirbus technology $\mathrm{GmbH}$ ). Liquid media (without antibiotics) were stored at room temperature. Solidified media (with or without antibiotics) were stored at $4{ }^{\circ} \mathrm{C}$.

\begin{tabular}{|l|c|}
\hline Compound & Concentration \\
\hline LB (lysogeny broth) Medium & $10 \mathrm{~g} \mathrm{I}^{-1}$ \\
\hline Tryptone & $5.0 \mathrm{~g} \mathrm{I}^{-1}$ \\
\hline Bacto Yeast extract & $10.0 \mathrm{~g} \mathrm{l}^{-1}$ \\
\hline $\mathrm{NaCl}$ & $20.0 \mathrm{~g} \mathrm{I}^{-1}$ \\
\hline Micro-Agar (Duchefa Biochemie B.V.) & \\
\hline pH 7.0 adjusted with NaOH & \\
\hline & $5.0 \mathrm{~g} / \mathrm{l}$ \\
\hline YEB Medium & $1.0 \mathrm{~g} / \mathrm{l}$ \\
\hline Bacto Beef extract & $5.0 \mathrm{~g} / \mathrm{l}$ \\
\hline Bacto Yeast ectract & $5.0 \mathrm{~g} / \mathrm{l}$ \\
\hline Bacto Peptone & $0.3 \mathrm{~g} / \mathrm{l}$ \\
\hline Sucrose & $20.0 \mathrm{~g} / \mathrm{l}$ \\
\hline MgSO & \\
\hline Micro-Agar $7 \mathrm{H}_{2} \mathrm{O}$ & \\
\hline pH 7.0 adjusted with $\mathrm{NaOH}$ & \\
\hline
\end{tabular}

Then, the E. coli cells were transferred to a $2 \mathrm{ml}$ reaction tube. E. coli cells were recovered by incubation at $37^{\circ} \mathrm{C}$ for $1 \mathrm{~h}$ with constant shaking at $100 \mathrm{rpm}$ (orbital shaking incubator, Sanyo Gallenkamp PLC) in darkness. Then 10 to $200 \mu \mathrm{l}$ of E. coli cell solution was spread on solid LB medium containing appropriate antibiotics round in petri dishes (92 mm diameter) (Table 11). E. coli cells were grown over-night at $37^{\circ} \mathrm{C}$ in darkness. Positive transformants were identified via colony-PCR and correctness of sequences was validated by sequencing (as described in chapter 2.12.3). 
A. tumefaciens cells were transformed with electroporation as described for $E$. coli with minor changes: A. tumefaciens cells were transferred in electroporation cuvettes with $2 \mathrm{~mm}$ gap. The A. tumefaciens cells were electroporated at $2.5 \mathrm{kV}$ for $6.0 \mathrm{~ms}$. After the electric pulse, $1 \mathrm{ml}$ YEB medium (room temperature) was added and cells were recovered for $4 \mathrm{~h}$ at $28{ }^{\circ} \mathrm{C}$ at $100 \mathrm{rpm}$ constant shaking in darkness. After recovery, A. tumefaciens cells were spread on solid YEB medium with appropriate antibiotics (Table 11) and grown for 2 days at $28^{\circ} \mathrm{C}$ in darkness.

Table 11. Antibiotics used for E. coli and A. thumefaciens selection for the different backbone vectors. The antibiotic was added to the medium after autoclaving, when a temperature of 50 to $55^{\circ} \mathrm{C}$ was reached.

\begin{tabular}{|l|l|l|}
\hline Backbone Vector & Antibiotic (filter sterile) stock & Final Concentration \\
\hline pDONR201 & Kanamycin $\left(50 \mathrm{mg} \mathrm{ml}^{-1}\right)$ & $50 \mu \mathrm{gl}^{-1}$ \\
\hline pB7WGF2 & Spectinomycin $\left(100 \mathrm{mg} \mathrm{ml}^{-1}\right)$ & $100 \mu \mathrm{g} \mathrm{m}^{-1}$ \\
\hline pK7WG2 & Spectinomycin $\left(100 \mathrm{mg} \mathrm{ml}^{-1}\right)$ & $100 \mu \mathrm{gl}^{-1}$ \\
\hline pBCseGFPEE & Spectinomycin $\left(100 \mathrm{mg} \mathrm{ml}^{-1}\right)$ & $100 \mu \mathrm{gl}^{-1}$ \\
\hline
\end{tabular}

\subsubsection{Creating VALR (At4g31330) overexpression lines}

VALR (At4g31330) overexpression lines were generated using the Gateway Cloning System (Hartley et al., 2000; Katzen, 2007, Figure 4).

\subsubsection{Adding attB recognition sites to VALR CDS (coding sequence)}

Primers for amplification of the At4g31330 (VALR) CDS were designed in Geneious Prime (Biomatters, Ltd., Auckland, New Zealand, https://www.geneious.com). In order to amplify the whole $A t 4 g 31330 \mathrm{CDS}$, the binding region for the primers were chosen at the beginning (forward primer) and at the end (reverse primer) of the CDS. The sequences for the attB recognition sites were added to the sequences of the binding regions at the $5^{\prime}$ and $3^{\prime}$. In addition, the sequence for an HA-tag (Human influenza hemagglutinin - tag) was added at the $3^{\prime}$-end of the gene to be able to detect the protein 
via Western Blot analysis. The resulting primer sequences are "At4g31330_attB1_for" and „At4g31330_attB2_rev“ (Figure 7; Table 15)

The designed primer were obtained from Microsynth Seqlab (Goettingen, Germany) and used in a PCR reaction with $5 \mu \mathrm{l}$. thaliana root CDNA as template and Phusion DNA Polymerase as described in chapter 2.10.5.

The resulting amplicon was $801 \mathrm{bp}$ in size and consisted of the attB1-site, the At4g31330 (VALR) CDS, the HA-tag, a Stop-codon and the attB2-site. Successful amplification of the attB1-At4g31330 CDS-HA-attB2 construct was confirmed by gel-electrophoresis as described in chapter 2.10.7. The PRC product of the attB1-At4g31330 CDS-HA-attB2 construct was purified and the concentration and quality were determined using a NanoDrop as described in chapter 2.10.8.

A

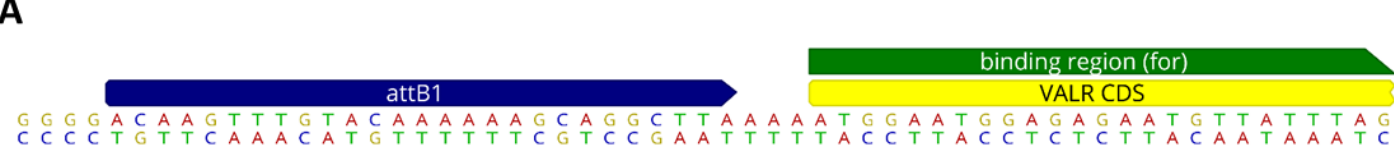

B

VALR CDS

AAAAGTCTAGTTTTGGATCTGTGTACCCATACGATGTTCCAGATTACGCTTAATACCCAGCTTTCTTGTACAAAGTGGTCCCC TinT CAGATCAAAACCTAGACACATGGGTATGCTACAAGGTCTAATGCGAATTATGGGTCGAAAGAACATGTTTCACCAGGGG

Figure 7. At4g31330 (VALR) primer sequences with attB-sites designed for the Gateway cloning system. A) forward primer "At4g31330_attB1_for". Binding region with attB1-site. B) reverse primer „At4g31330_attB2_rev“. Binding region with HA-tag, Stop-codon and attB2-site.

\subsubsection{BP reaction}

In order to insert the attB1-At4g31330 CDS-HA-attB2 construct in the donor vector pDONR201 (Thermo Fisher Scientific, Table 16), a BP reaction was performed (Figure 8A) using the Gateway BP Clonase II Enzyme mix (Invitrogen). Basically, the protocol "Gateway ${ }^{\oplus}$ Technology" provided by Invitrogen was followed. Equimolar amounts (50 fmol) of attB1-At4g31330 CDS-HA-attB2 construct and pDONR201 vector were mixed. The following formula was used to convert femtomoles (fmol) of DNA to nanograms (ng) of DNA: 
$\mathrm{ng}=(50 \mathrm{fmol})(\mathrm{N})\left(\frac{660 \mathrm{fg}}{\mathrm{fmol}}\right)\left(\frac{1 \mathrm{ng}}{10^{6} \mathrm{fg}}\right)$ where $\mathrm{N}$ is the size of DNA in bp. The attB1-At4g31330 CDS-HA-attB2 construct-pDONR201 vector mix was adjusted to a final volume of $8 \mu$ l with TE buffer (Table 4).

Then, $2 \mu$ B BP Clonase II enzyme mix was added, and the cloning reaction was incubated at $25^{\circ} \mathrm{C}$ over-night in a thermocycler (Labcycler Gradient, Sensoquest). The reaction was terminated by adding $1 \mu \mathrm{l}$ of Proteinase $\mathrm{K}$ solution and incubating the mixture at $37^{\circ} \mathrm{C}$ for $10 \mathrm{~min}$ in a thermocycler (Labcycler Gradient, Sensoquest). Through ligation of the attB1-At4g31330 CDS-HA-attB2 construct into pDONR201, the attB-sites turned into attL-sites, creating the attL1-At4g31330 CDS-HA-attL2-pDONR201 entry clone (Table 16).

\subsubsection{Selection and multiplication of the successfully cloned attL1-VALR CDS-HA-attL2-pDONR201 entry clone}

Fifty $\mu$ l of electrocompetent $E$. coli DH5 $\alpha$ cells (chapter 2.12.1) were mixed with $1 \mu$ l of the created attL1-At4g31330 CDS-HA-attL2-pDONR201 entry clone and transformation was performed as described in chapter 2.12.2. Then, $100 \mu$ of $E$. coli were spread on solid LB medium supplemented with $50 \mu \mathrm{g} / \mathrm{ml}$ Kanamycin. E. coli colonies were grown for 20 to $24 \mathrm{~h}$ at $37^{\circ} \mathrm{C}$ at darkness in an incubator (Sanyo Gallenkamp PLC).

Under these conditions non-transformed E. coli cells die, because of the missing Kanamycin resistance. E. colis, transformed with the empty pDONR201 vector, produce lethal CcdB toxin of Type II Toxin-antitoxin system and die as well. The responsible toxin gene is encoded on the donor vector and replaced by the gene of interest during cloning (Figure 8A).

For the colony PCR, 7 colonies were picked separately with a sterile pipette tip and mixed with a Taq-PCR reaction (chapter 2.10.8; Table 6). The residual E. coli cells on the tip were transferred on new selection medium, numbered consecutively, and grown at $37^{\circ} \mathrm{C}$ in darkness.

The colony-PCR was performed with a gene-binding primer (At4g31330_attB1_for) and a plasmid-binding primer (pDONR_rev) to confirm VALR CDS-insertion into pDONR201 (Table 15). Successful amplification of the attL1-At4g31330 CDS-HA-attL2-pDONR201 construct was confirmed by gel-electrophoresis as described in chapter 2.10.7. 
After incubation of the picked E. coli colonies, one positively transformed colony was used to inoculate $7 \mathrm{ml}$ liquid LB medium with Kanamycin $(50 \mu \mathrm{g} / \mathrm{ml})$. Bacteria were grown overnight at $37^{\circ} \mathrm{C}$ while shaking ( $250 \mathrm{rpm}$; orbital shaking incubator (Sanyo Gallenkamp PLC)). Then, the attL1-At4g31330 CDS-HA-attL2-pDONR201 entry clone was extracted from the E. coli $\mathrm{DH} 5 \alpha$ culture as described in chapter 2.10.8.

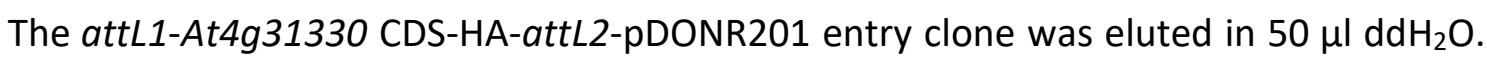
The concentration and quality were determined using a NanoDrop as described in chapter 2.10.8. Approximately $80 \mathrm{ng} \mathrm{\mu l}^{-1}$ of the attL1-At4g31330 CDS-HA-attL2-pDONR201 entry clone were provided for sequencing at Microsynth Seqlab (Goettingen, Germany) with pDONR_for and pDONR_rev primer (Table 15). Sequences were examined in Geneious Prime (Biomatters, Ltd., Auckland, New Zealand, https://www.geneious.com) to confirm correctness.

\subsubsection{LR Reaction}

In the next step, an expression clone was generated. For this purpose, the attL1-At4g31330 CDS-HA-attL2-pDONR201 entry clone generated in chapter 2.12.3.3, was mixed in the LR reaction either with the destination vector pK7WG2 to create the p35S::At4g31330-HA expression clone or with pB7WGF2 to generate the p35S::eGFP-At4g31330-HA expression clone (Karimi et al., 2002; VIB, Gent, Belgium) as described below in this chapter (Figure 8B; Table 16). Both vectors contain the constitutive $35 \mathrm{~S}$ promotor from the cauliflower mosaic virus, which allows overexpression of the introduced gene (Benfey and Chua, 1990; Odell et al., 1985). The pB7WGF2 vector has an N-terminal eGFP protein.

The LR reaction was performed with the Gateway LR Clonase II Enzyme Mix (Invitrogen) following the protocol of the manufacturers with minor changes. Briefly, $150 \mathrm{ng}$ of each vector were mixed with TE buffer to a final volume of $8 \mu$ l (Table 4). $2 \mu$ l of LR Clonase II enzyme mix was added and the reaction was incubated at $25^{\circ} \mathrm{C}$ overnight. Then, $1 \mu \mathrm{l}$ Proteinase $\mathrm{K}$ was added and inactivation of $\mathrm{LR}$ reaction occurred at $37^{\circ} \mathrm{C}$ for $20 \mathrm{~min}$. The resulting expression clones are p35S::At4g31330-HA and p35S::eGFP-At4g31330-HA (Table 16). 
2.12.3.5 Selection and multiplication of the successfully cloned p35S::At4g31330-HA and p35S::eGFP-At4g31330-HA expression clones

A scheme of the procedure is shown in Figure 43. One $\mu l$ of the LR-expression clone mixture was used to transform $50 \mu \mathrm{l}$ E. coli DH5 $\alpha$ cells as described in chapter 2.12.2. For colony selection, LB medium supplemented with $100 \mu \mathrm{g} / \mathrm{ml}$ Spectinomycin was used. After overnight incubation at $37^{\circ} \mathrm{C}$ in darkness, colony PCR with Taq-polymerase and pK7WG_rev and pK7WG_for primer was performed as described in chapter 2.10.8 (Table 6 and Table 15).

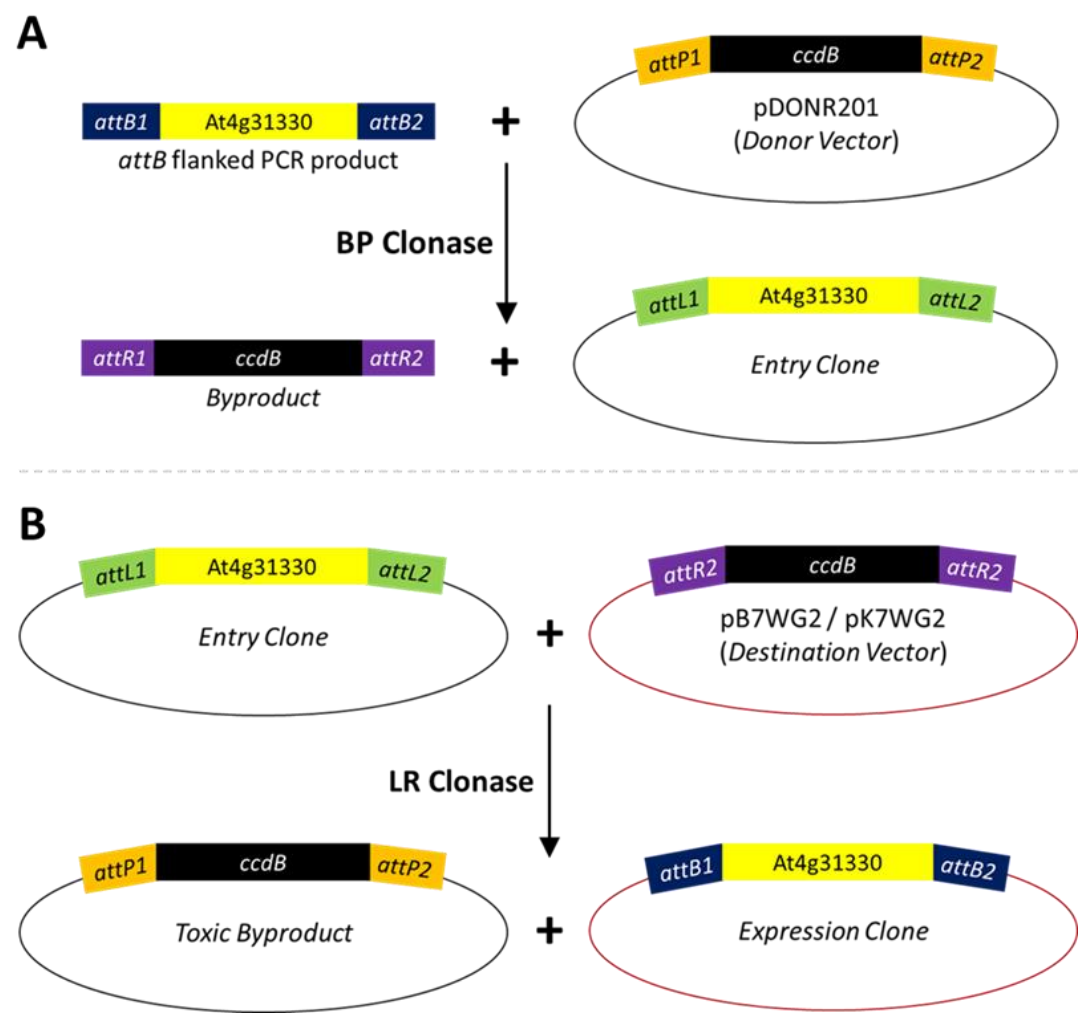

Figure 8. Gateway system for At4g31330 overexpression line. A) BP reaction leads to an attL flanked entry clone. B) The LR reactions leads to an expression clone containing all necessary components for constitutive gene expression

The amplified PCR products were monitored on an Agarose gel to confirm successful cloning of the p35S::At4g31330-HA and p35S::eGFP-At4g31330-HA as described in chapter 2.10.7. Positively transformed colonies were grown overnight in liquid culture (LB medium with Spectinomycin) and plasmids were extracted as described above (chapter 2.12.3.3). The extracted p35S::At4g31330-HA and p35S::eGFP-At4g31330-HA expression clones were eluted in $60 \mu \mathrm{lddH_{2 }} O$ each. Concentration and quality were 
measured spectrophotometrically as described in chapter 2.10.8. Approximately,

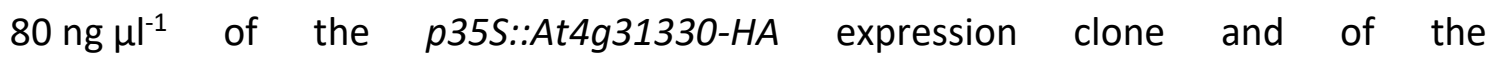
p35S::eGFP-At4g31330-HA expression clone were provided for sequencing at Microsynth Seqlab (Goettingen, Germany) with pK7WG_for and pK7WG_rev primer (Table 15). Sequences were examined in Geneious Prime (Biomatters, Ltd., Auckland, New Zealand, https://www.geneious.com) to confirm sequence correctness.

The extracted p35S::At4g31330-HA and p35S::eGFP-At4g31330-HA expression clones with a correct sequence were then used to transform $A$. tumefaciens as described in chapter 2.12.2.
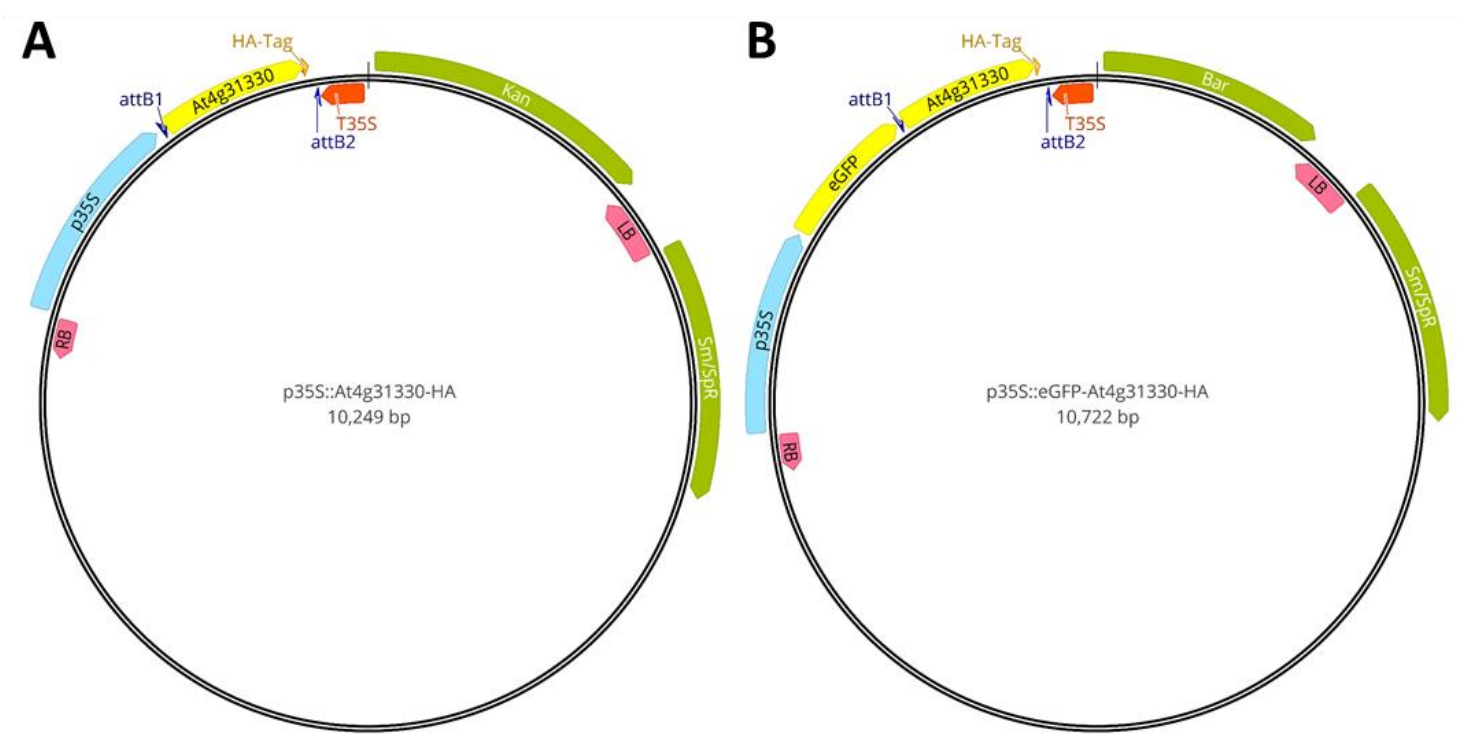

Figure 9. At4g31330 expression clones. A) Resulting expression clone with the Gateway vector pK7WG2. At4g31330 is controlled by the consitutive p35S promotor and contains a HA-tag at the $3^{\prime}$ end. B) Expression clone with the gateway vector pB7WGF2. The p35S pomotor drives expression of eGFP, At4g31330 and the HA tag. T35S, 35S-Terminator; RB, T-DNA right border; LB, T-DNA left border; Kan, Kanamycin resistance; Bar, Basta resistance (Glufosinate-ammonium) Sm, Spectinomycin resistance; Sp, Streptomycin resistance. Maps created with Geneious Prime.

\subsubsection{Targeted deletion of VALR (At4g31330) for loss- or reduction-of-function mutagenesis}

In order to generate At4g31330 loss- or reduction-of-function, CRISPR/Cas9 was used. Cloning was performed according to Wang et al. (2015) and Xing et al. (2014) with some 
modifications as described below. With the goal of deleting the whole VALR (At4g3330) gene, two CRISPR/Cas9 target sites, one at the beginning, and one at the end of the gene were chosen (Figure 10A). For each target site, two oligo primer were designed using Geneious Prime (Biomatters, Ltd., Auckland, New Zealand, https://www.geneious.com), CRISPRdirect (Naito et al., 2015; http://crispr.dbcls.jp/) and CRISPR-P v2.0 (http://crispr.hzau.edu.cn). One target site in the At4g31330 gene was defined as $20 \mathrm{bp}$ of the DNA sequence followed by the protospacer adjacent motif (PAM; 5'-NGG-3') containing 50 to $60 \%$ guanin and cytosine (Figure 10B). The sequences must start with guanin (at the $5^{\prime}$-end). If it was not available, a guanine was added to the oligo sequence. Furthermore, a Bsal restriction site was added to each oligo sequence to be able to clone the amplified PCR product into the CRISPR/Cas9 vector pBCsGFPEE (Figure 10C and D, Table 16). In addition, the oligo sequences contained binding sites of the guideRNA (gRNA) and U6-29 promotor to be able to bind to the template CRISPR/Cas9 plasmid pBCSGEE-ROXY11-15BA (produced and provided by Dr. Thurow; Table 16) (Figure $10 \mathrm{C}$ and D). Primer were ordered and diluted as described in chapter 2.10.6. PCR with Phusion-DNA-Polymerase was performed with $10 \mathrm{ng}$ of pBCSGEE-ROXY11-15BA as template as described in chapter2.10.6. In order to enhance the chance for successful At4g31330 gene deletion, each forward-reverse primer combination was used (T1 and $\mathrm{T} 3, \mathrm{~T} 1$ and T4, T2 and T3, T2 and T4, Figure 10A). The resulting PCR product contained the CRIPSR/Cas9 target site 1 of At4g31330, the guide RNA sequence, the U6-26 promotor terminator, the U6-29 promotor and the CIRSPR/Cas9 target site 2 of At4g31330. It was embedded between two Bsal restriction sites (Figure 11A).

After PCR product purification as described in 2.10.8, Golden Gate cloning (Engler et al., 2008) was performed according to manufacturer's protocol (New England Biolabs) with T4 ligase and the Bsal-Hv2 restriction enzyme to insert the PCR product into the CRISPR/Cas9 vector pBCsGFPEE (Table 12; Table 16). A molar vector:insert (pBCsGFPEE:PCR product) ratio of 1:3 was used. Restriction and ligation occurred at $37^{\circ} \mathrm{C}$ for $5 \mathrm{~min}, 16^{\circ} \mathrm{C}$ for $5 \mathrm{~min}$, repeated for $30 \mathrm{cycle}$, followed by a final ligation at $16{ }^{\circ} \mathrm{C}$ overnight and an inactivation at $60^{\circ} \mathrm{C}$ for $5 \mathrm{~min}$ in a thermocycler (Labcycler, Sensoquest). Then, the reaction was diluted $1: 5$ by adding $80 \mu \mathrm{ldd} \mathrm{H}_{2} \mathrm{O}$. From this dilution, $10 \mu \mathrm{l}$ were used to transform $50 \mu \mathrm{l}$. coli DH5 $\alpha$ cells as described in chapter 2.12.2. 
A

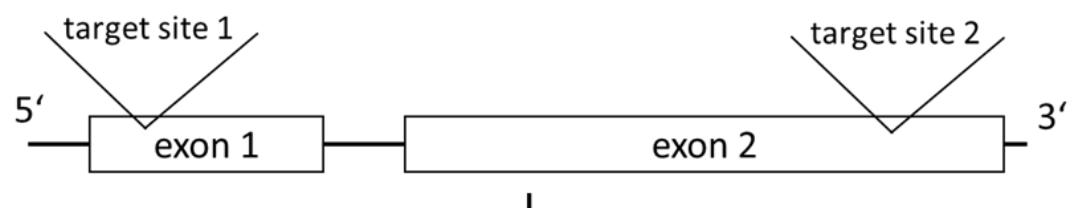

two oligos per

target site

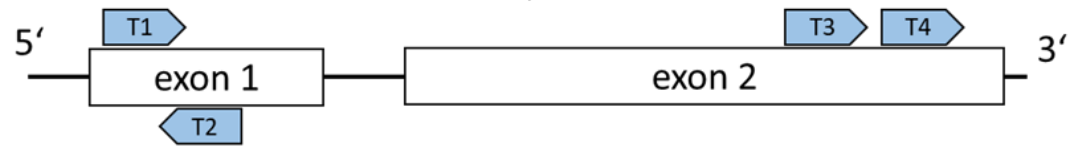

B

$\mathrm{T}>$ 5'-NNNNNNNNNNNNNNNNNNNNNGG-3'
$20 \mathrm{bp}$

C

\begin{tabular}{|c|cc|c|c|}
\hline T1 & ATATATGGTCTCTGATTGNNNNNNNNNNNNNNNNNNNGTTTTAGAGCTAGAAATAGCAAG \\
\hline $\mathrm{T} 2$ & Bsal & $20 \mathrm{bp}$ & part of gRNA
\end{tabular}

T3 ATTATTGGTCTCTAAACNNNNNNNNNNNNNNNNNNNNCAATCTCTTAGTCGACTCTACC

T4 Bsal $20 \mathrm{bp} \quad$ part of U6-29p

D

\begin{tabular}{|c|c|c|c|}
\hline T1 & $\begin{array}{r}\text { ATATATGGTC } \\
\text { Bsal }\end{array}$ & $\begin{array}{l}\text { ATTGGT } \\
20 \mathrm{bp}\end{array}$ & $\frac{\text { AATAGCAAG }}{\text { part of gRNA }}$ \\
\hline T2 & $\begin{array}{r}\text { ATATATGGTC } \\
\text { Bsal }\end{array}$ & $\begin{array}{l}\text { CATTAG } \\
20 \mathrm{bp}\end{array}$ & AATAGCAAG \\
\hline
\end{tabular}

T3 ATTATTGGTCTCTAAACTCTCTCTAGCAACTCCGCCACAATCTCTTAGTCGACTCTACC

Bsal $20 \mathrm{bp} \quad$ part of U6-29p

T4 ATTATTGGTCTCTAAACCGTAGAACAACCGATTCCCCAATCTCTTAGTCGACTCTACC

Bsal $20 \mathrm{bp} \quad$ part of U6-29p

Figure 10. Design of CRISPR/Cas9 target sites and oligonucleotides. A) Scheme of At4g31330 gDNA. Target sites were chosen at the beginning and the end of the gene. For each target site, two target-oligonucleotides were designed (T1, T2, T3 and T4). One oligonucleotide contained $20 \mathrm{bp}$, which were in front of a PAM-site (B). C) Designed oligonucleotides. The PCR products needed to be embedded by Bsal restriction sites. Furthermore, template specific binding sites (parts of gRNA and U6-29p) ensured amplification of the T1-gRNA-U6-26t-U629p-T2 construct. Red marked bp are necessary. If the 20 bp target sequences do not start or end with this $\mathrm{G}$ or $\mathrm{C}$, these base pairs are added to the oligonucleotide sequence. 
Table 12. Golden Gate Cloning to ligate the CIRSPR/Cas9 backbone pBCsGFPEE with the At4g31330-target sites construct (Figure 11). The protocol provided by New England Biolabs was followed.

\begin{tabular}{|l|r|}
\hline Compound & Volume \\
\hline pBCsGFPEE vector $(55 \mathrm{fmol})$ & $600.0 \mathrm{ng}$ \\
\hline PCR-product with target sites $(165 \mathrm{fmol})$ & $70.0 \mathrm{ng}$ \\
\hline T4 Ligase Buffer & $2.0 \mu \mathrm{l}$ \\
\hline Bsal-HFv2 $(20 \mathrm{U} / \mu \mathrm{l})$ & $1.5 \mu \mathrm{l}$ \\
\hline T4 Ligase $(400 \mathrm{U} / \mu \mathrm{l})$ & $2.5 \mu \mathrm{l}$ \\
\hline $\mathrm{dd}_{2} \mathrm{O}$ & ad to $20 \mu \mathrm{l}$ \\
\hline
\end{tabular}

After transformation, E. coli was grown on solid LB medium supplemented with Spectinomycin $(100 \mu \mathrm{g} / \mathrm{ml})$ at $37^{\circ} \mathrm{C}$ in darkness overnight. Then, colony PCRs were performed with Taq-Polymerase and the respective target site primers (At4g31330_T1-gRNA-Bsal-fwd, At4g31330_T2-gRNA-Bsal-fwd, 29p-At4g31330_T3-gRNA-Bsal-rev, 29p-At4g31330_T4-gRNA-Bsal-rev) to identify clones transformed with the pBScGFPEE-At4g31330-target sites construct (Figure 11B) as described in chapter 2.10.6 (Table 15). Three plasmids could have been transformed: 1. the template plasmid pBCsGEE-ROXY11-15BA, 2. the empty CRISPR/Cas9 backbone pBCsGFPEE and 3. the pBScGFPEE with the successfully inserted At4g31330-target sites-PCR product, the desired construct (Figure 11B).

To be able to distinguish between those plasmids, a 2-step and a 3-step PCR were executed. Since the target site primers are long (Table 15), they can anneal at $72{ }^{\circ} \mathrm{C}$ in the 2-step PCR in contrast to vector or gene specific primers which require a temperature of $50{ }^{\circ} \mathrm{C}$. Conditions for the 2-step-PCR were: initial denaturation at $94{ }^{\circ} \mathrm{C}$ for $3 \mathrm{~min}$, followed by 35 cycles 1 ) denaturation at $94{ }^{\circ} \mathrm{C}$ for $30 \mathrm{~s}$ and 2) elongation at $72{ }^{\circ} \mathrm{C}$ for $1 \mathrm{~min}$, and final elongation at $72{ }^{\circ} \mathrm{C}$ for $5 \mathrm{~min}$. As controls, 3-step PCRs were performed with an annealing temperature of $50{ }^{\circ} \mathrm{C}$ with the empty vector $\mathrm{pBCsGFPEE}$, the target site PCR product and pBCsGFPEE-ROXY11-15-BA as templates (chapter 2.10.6). For pBCsGFPEE as template, no PCR product is expected, because the primers cannot bind. For the desired plasmid (pBScGFPEE-At4g31330-target sites construct), a PCR product in both, the 2-step and the 3-step PCRs is expected. 
If pBCsGFPEE-ROXY11-15-BA was transformed, the 3-step PCR with annealing at $50^{\circ} \mathrm{C}$ resulted in a product, whereas the two-step PCR did not result in a product, because the annealing temperature was too high. Thus, the 2-step colony PCR with the target site primers and an annealing temperature of $72{ }^{\circ} \mathrm{C}$ only results in a PCR product, if the desired pBScGFPEE-At4g31330-target sites construct is successfully transformed. Successful amplification was confirmed with gel-electrophoresis as described in chapter 2.10.7. Successfully transformed colonies were found for the At4g31330 target site combinations T1-T4, T1-T3 and T2-T4 (Figure 10). For the T1-T3 combination, no successfully transformed E. coli clone was found (Figure 10).

Successfully transformed colonies were grown in liquid LB medium for plasmid extraction (chapter 2.10.8). The extracted plasmids were sent for sequencing to Microsynth Seqlab (Goettingen, Germany) using the primer V69-pXCS-for or V69-pXCS-rev (Table 15). The resulting sequences were analyzed in Geneious Prime (Biomatters, Ltd., Auckland, New Zealand, https://www.geneious.com) to confirm correct cloning.

The pBScGFPEE-At4g31330-target sites construct (the At4g31330-CRIPSR/Cas9 plasmid) was used to transform A. tumefaciens (chapter 2.12.2). A. tumefaciens was grown on solid YEB medium supplemented with Rifampicin, Gentamycin and Spectinomycin (Table 11 ) at $28^{\circ} \mathrm{C}$ in darkness for 2 days.

Colony PCR, plasmid extraction, and sequencing was performed as described above and in chapters 2.10.6 and 2.10.8. Positive clones which were used to transform A. thaliana.

\subsubsection{Stable transformation of $\boldsymbol{A}$. thaliana using the "Floral dip" method}

A. thaliana were transformed with the "floral dip" method (modified from Clough and Bent, 1998). Transformed A. tumefaciens created in chapter 2.12.2 and 2.12.4 was grown in $10 \mathrm{ml}$ YEB medium supplemented with the appropriate antibiotics (Table 11) at $28{ }^{\circ} \mathrm{C}$ overnight while shaking at $90 \mathrm{rpm}$ in an orbital shaking incubator (Sanyo Gallenkamp PLC). 
A

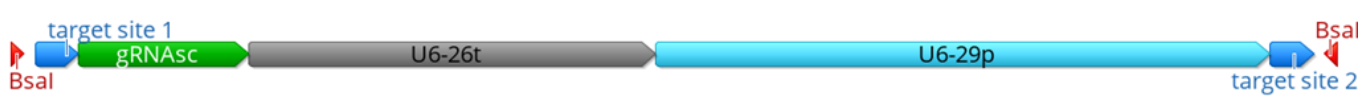

B

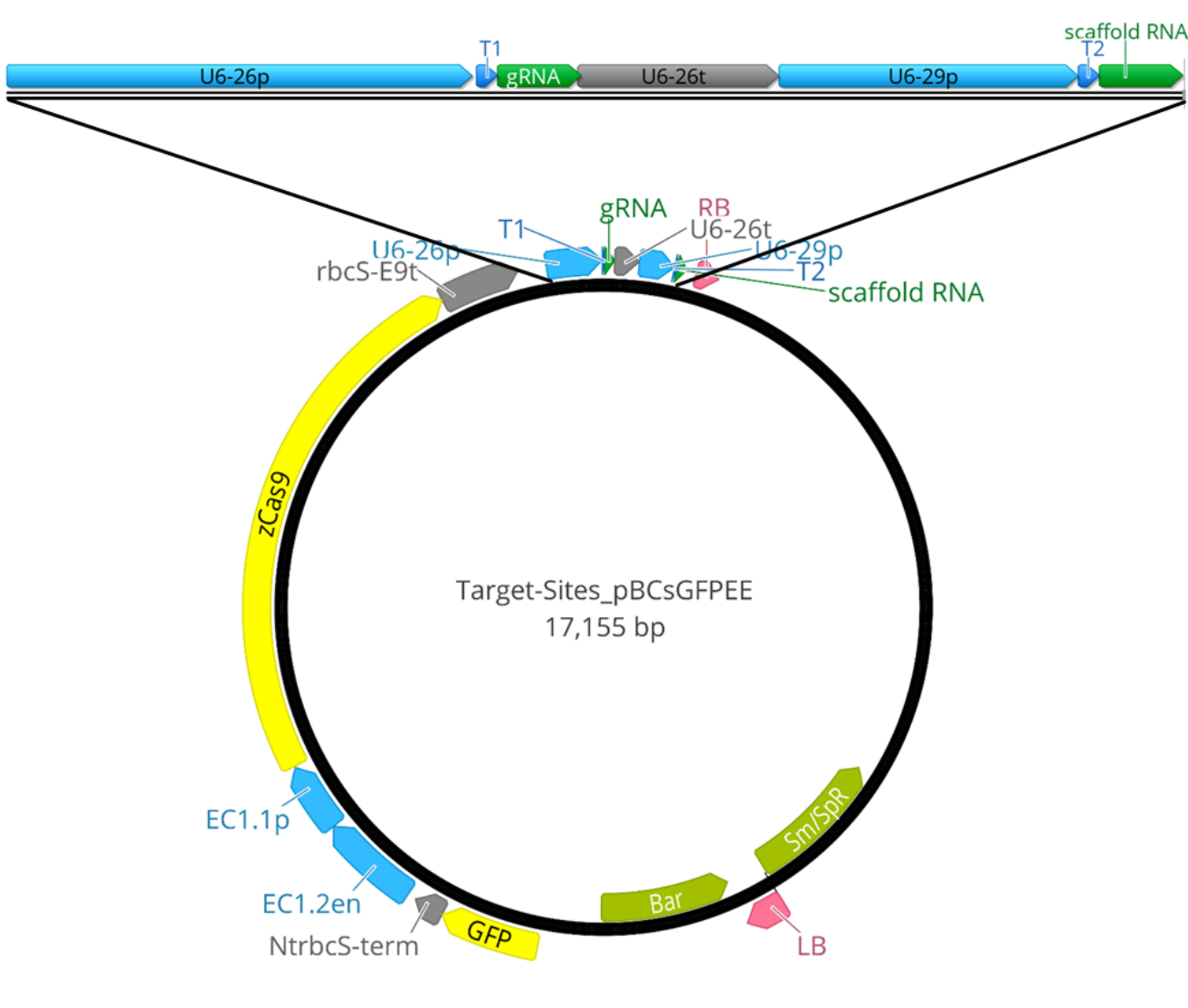

Figure 11. A) CRIPSR/Cas9-At4g31330 target sites PCR product. B) pBCsGFPEE with At4g31330 CRISPR/Cas9 target sites. The target sites are driven by Pol-III promoters $\mathrm{U}-26 \mathrm{p}$ and U6-29p. Cas9 is driven by the egg-cell specific promotor EC1.1p enhanced by the EC1.2en enhancer. rbcS-E9t, rbcS E9 terminator; NtrbcS-term, nost rbcS terminator; gRNA, guide RNA; Sm, Spectinomycin resistance; Sp, Streptomycin resistance; GFP, green fluorescent protein; Bar, Basta resistance (Glufosinate-Ammonium); RB, T-DNA right border; LB, T-DNA left border.

The next day, $5 \mathrm{ml}$ of the overnight culture was transferred to a 2 I baffled flasks with $500 \mathrm{ml}$ YEB medium with antibiotics (Table 11). In total, $1 \mathrm{~L}$ A. tumefaciens culture in two baffled flasks, each filled with $500 \mathrm{ml}$ YEB medium with antibiotics was prepared. A. tumefaciens was grown overnight at $28^{\circ} \mathrm{C}$ and shaking at $90 \mathrm{rpm}$ in an orbital shaking incubator (Sanyo Gallenkamp PLC). Then, the culture was divided to four $250 \mathrm{ml}$ centrifugation beakers and centrifuged for $10 \mathrm{~min}$ at $5000 \mathrm{~g}$ at room temperature. 
The supernatant was discarded and the cell pellet was re-suspended in $250 \mathrm{ml}$ $5 \%$ sucrose. The bacterial suspension was transferred to glass jars (provided by J. Weck GmbH u. Co. KG), The suspension was supplemented with $50 \mu$ l Silwett L-77 (Lehle Seeds) to reduce the surface tension. Four to five-week-old $A$. thaliana plants with flowers and buds grown under the described conditions in chapter 2.1.1 were prepared by covering them with a plastic dome overnight to enhance humidity the day before transformation. Plants were dipped up site down into the bacterial solution for three to five seconds ensuring that flowers and buds were in contact with the suspension. Then, plants were placed back in the trays and covered with dome to keep the humidity high until the plants straightened up. After two days, the plants were kept in the laboratory at room temperature and constant light.

The dome was removed, and shoots were covered in paper bags and grown in a climate chamber at long-day conditions (chapter 2.1.1). They were watered for two weeks and then watering stopped and plants dried to harvest mature F1 generation seeds.

\subsubsection{Selection of positively transformed $A$. thaliana plants}

\subsubsection{Selection of VALR (At4g31330) overexpression lines}

A scheme of the procedure is shown in Figure 49. A. thaliana F1 seeds (the progeny of F0 plants) from the overexpression lines (chapter 2.12.3 and 2.12.4), were harvested, sterilized and stratified as described in 2.1.4. Then, they were placed on AM medium without sucrose and MES, containing either Kanamycin $\left(50 \mu \mathrm{g} \mathrm{ml}^{-1}\right)$ for pK7WG2-constructs (p35S::At4g31330-HA) or glufosinate-ammonium (15 $\mathrm{g} \mathrm{ml}^{-1}$ ) for pB7WGF2-constructs (p35S::eGFP-At4g31330-HA) (Table 1). After 10 to 14 days of growth on the selection medium under long day conditions only transformed seedlings grew, wildtype seedlings died because of the missing antibiotic resistance (chapter 2.1.1). All grown seedlings were transferred in Type $\mathrm{N}$ soil and covered with the plastic dome for approximately one week (chapter 2.1.1). Then, the dome was removed. Leaves from 3 to 4-week-old plants were harvested for genotyping. DNA was extracted and T-DNA insertion was confirmed via PCR using a gene specific primer (AtDUF599_for_ex1) and a plasmid binding primer (pK7WG2_rev) as described in chapter 2.10.1 and 2.10.6. Successfully transformed plants were grown for 6 to 8 weeks 
under the conditions described in chapter 2.1.1. Then, watering was stopped to dry the plants and harvest the matured progeny of F1 plants.

The selection-procedure was repeated for the F1 progeny to grow positive transformed F2 generation plants as described above for F1 generation plants.

From the most promising three F2 lines, seeds were harvested. In order to identify homozygous F2 plants, the progeny of 6 F2 plants was sterilized and stratified as described in chapter 2.1.4 and placed on selective AM to determine the growth rate (Table 1). As control, seeds from each F2 plant were also grown on AM medium without antibiotics (Table 1). According to Mendelian segregation, $25 \%$ of the seeds originated from a heterozygous F2 plant are untransformed (wildtype), $50 \%$ are heterozygous for the transformed p35S::At4g31330-HA or the p35S::eGFP-At4g31330-HA construct and $25 \%$ are homozygous for the transformed construct. Thus, a growth rate of $75 \%$ on selective AM medium was expected for the progeny of a heterozygous F2 plant. The expected growth rate for the progeny of a homozygous F2 plant on selective AM was $100^{\circ} \%$. In addition, the gene transcription of the homozygous $\mathrm{T} 2$ plants was investigated with qRT-PCR as described in chapter 2.10.9.

\subsubsection{Selection of VALR (At4g31330) potential loss-of function lines}

A. thaliana F1 seeds (the progeny of F0 plants) from the potential loss-of function lines produced with the CRISPR/Cas9 system where checked for T-DNA insertion (chapter 2.12.4). For this purpose, the progeny of FO plants was selected on AM without sucrose supplemented with glufosinate-ammonium $\left(15 \mathrm{mg} \mathrm{l}^{-1}\right)$ for 10 to 14 days (Table 1). Grown seedlings were transferred to soil and grown as described in chapter 2.1.1. Leaf samples from 3 to 4-week-old F1 plants were used for genotyping. DNA was extracted with the CTAB method (chapter 2.10.1.2) and used for PCR with Taq-DNA-Polymerase (chapter 2.10.6). One PCR was performed with gene specific primers (AtDUF599for_ex1 and AtDUF599rev_ex2N; Table 15) to amplify the gene and to investigate whether it was deleted by the Cas9 enzyme. Deletion happens if the Cas 9 enzyme cuts in both target sites, in the beginning and the end of the gene (Figure 11A). A second PCR was performed with plasmid specific primers (V96-pXCS-for and V96-pXCS-rev; Table 15) to investigate the T-DNA insertion of the 
pBScGFPEE-At4g31330-target sites construct (Table 16; chapter 2.12.4). In F1 plants the presence of the T-DNA is expected. Successful amplification was confirmed with gel electrophoresis (chapter 2.10.7). Each PCR product amplified with At4g31330 gene specific primers had the same size as the PCR product with wildtype DNA. Thus, none of the F1 plants had a gene deletion. The T-DNA was detected in 10 of 14 plants transformed with the T1-T4 target sites and in 6 of 14 plants transformed with the T1-T3 target sites (chapter 2.12.4).

To detect base pair insertions or deletions (indels), a mixed T7 endonuclease 1 (T7E1) assay was performed. T7 endonuclease detects indels within a gene compared to a template strand and cuts the DNA at the respective site. To detect indels, a PCR with Phusion-polymerase was performed using genomic DNA extracted from either mutant or wildtype leaves as template (chapter 2.10.6). Primers pAt4g31330_for and AtDUF599rev_ex2N (Table 15) were used to enclose both target sites in the PCR product. Two point five $\mu$ l of the resulting PCR product of mutant DNA was mixed with $2.5 \mu \mathrm{IPCR}$ product of wildtype DNA to be able to detect homozygous mutants. To anneal both products, the PCR product mixture $(5 \mu \mathrm{l})$ was denatured at $95^{\circ} \mathrm{C}$ for $5^{\circ} \mathrm{min}$ and then annealed by cooling to $15^{\circ} \mathrm{C}$ with a ramp rate of $0.1{ }^{\circ} \mathrm{C} \mathrm{s}^{-1}$ in a thermocycler (Labcycler, Sensoquest). Annealing resulted in a heteroduplex of which $5 \mu$ l were mixed with $6 \mu \mathrm{l}$ of a mastermix according to Table 13 . The digestion occurred at $37^{\circ} \mathrm{C}$ for $1 \mathrm{~h}$ in a thermocycler (Labcycler, Sensoquest). The digestion was analyzed with gel-electrophoresis using a $2 \%$ Agarose, loaded with $11 \mu \mathrm{l}$ per well. If the mutant contained an indel the digestion resulted in 3 bands of different sizes. The largest band display the wildtype PCR product and the two lower ones display the two products that originate from the $\mathrm{T7}$ endonuclease restriction.

After the mixed T7E1 assay (PCR products from wildtype DNA as template mixed with PCR product from mutant DNA), the PCR product from most promising mutated plant lines were further analyzed in a T7E1 assay in which the mutant PCR product was not mixed with wildtype PCR product. If cleavage products were detected again, the corresponding DNA was heterogeneous and thus the corresponding F1 plant was heterozygote for the indel. If the product was not cleaved in the second assay but in the first, the indel mutation is most likely homogeneous and thus the corresponding F1 plant 
was homozygote for the indel. Since all of the tested plants showed a cleaved product, the PCR product of the F1 lines 4 and 6 transformed with the target sites T1 and T4 (valr4 and valr6) and the line 1 transformed with the target sites T1 and T3 (valr1) were purified as described in chapter 2.10.8 and sent for sequencing with the primers AtDUF599for_ex1 and AtDUF599rev_ex2N (Table 15) to Microsynth Seqlab (Goettingen, Germany). The resulting sequences were compared to the reference sequence in Geneious Prime (Biomatters, Ltd., Auckland, New Zealand, https://www.geneious.com) and the chromatograms were analyzed with Chromas (Technelysium Pty Ltd, South Bisbane, Australia, https://technelysium.com.au/wp/chromas). Line valr4 and valr6 were indeed heterozygote. Line valr1 was a homozygote wildtype.

After seed maturation of F1 plants, they were harvested from most promising primary plants (heterogeneous or homogenous for frameshifts or insertions and deletions). From each construct (At4g31330 target site T1 and T3 and target site T1 and T4 (chapter 2.12.4)), at least 6 independent lines were harvested and further analyzed.

The seeds were examined with a fluorescence stereomicroscope provided by Leica Microsystems GmbH (M205FA) and checked for their fluorescence. The pBCsGFPEE CRIPSR/Cas9 backbone contains a GFP encoding sequence regulated by a seed specific promoter (Figure 11B). Seeds without the CRISPR/Cas9 construct have inherited their alleles from their parents and passed them stably to the next generation via germline so that the presence of the CRIPSR/Cas9 is no longer necessary.

Therefore, seeds without fluorescence were selected for growth. From each construct, 20 seeds without fluorescence from 6 lines were selected and put on soil (Fruhstorfer Erde Type N, Hawita, Vechta, Germany) in Arasystem tray with a pot diameter of $5 \mathrm{~cm}$ provided by BETATECH BVBA (Belgium). In addition, 5 fluorescent seeds of each line were selected for growth. Furthermore, seeds from four segregated wildtype plants (two per construct), among them was valr1, were grown.

The trays were covered with transparent domes. The seeds were stratified on soil in the cooling room $\left(4^{\circ} \mathrm{C}\right)$ for two days and then grown in a climate chamber under the conditions described in chapter 2.1.1. As soon as the leaves were large enough, samples were taken for DNA extraction and genotyping with the T7E1 assay as described above. Furthermore, all candidate plants were investigated via PCR for the loss of their 
CRISPR/Cas9 containing T-DNA using Taq-DNA-polymerase and the V96-pXCS-primer pair (Table 15) as described in chapter 2.10.6. PCR products of promising lines were extracted (chapter 2.10.8) and sent for sequencing to Microsynth Seqlab (Goettingen, Germany). The resulting sequences were compared to the reference sequence in Geneious Prime (Biomatters, Ltd., Auckland, New Zealand, https://www.geneious.com) and the chromatograms were analyzed with Chromas (Technelysium Pty Ltd, South Bisbane, Australia, https://technelysium.com.au/wp/chromas). Line valr4-8 and valr6-1 and were homozygote for a T-insertion in the T4 target site and lost the CRISPR/Cas9 construct. Line valr1-5 was heterozygote for a gene deletion and inherited the CRISPR/Cas9.

After seed maturation of F2 plants, the seeds were harvested. The seeds of valr1-5 F2 plants were investigated with a fluorescence stereomicroscope as described above. No and weak fluorescent seeds were selected and grown as described above. As soon as leaves of the valr1-5 F3 plants were large enough, some were harvested for genotyping. DNA was extracted as described in chapter 2.10.1.2. PCR with Phusion polymerase and pAt4g31330_for and At4g31330_3'UTR_rev primer (Table 15) was performed as described in chapter 2.10.6. The PCR with mutant DNA as template resulted in a shorter amplicon compared to the wildtype amplicon indicating deletion of theA At4g31330 gene. The PCR product was purified and sent for sequencing to Microsynth Seqlab (Goettingen, Germany)(chapter 2.10.8). The resulting sequences were compared to the reference sequence in Geneious Prime (Biomatters, Ltd., Auckland, New Zealand, https://www.geneious.com) and the chromatograms were analyzed with Chromas (Technelysium Pty Ltd, South Bisbane, Australia, https://technelysium.com.au/wp/chromas). The sequence analysis confirmed that valr1-5-1 and valr1-5-9 were homozygote for the At4g31330 gene deletion. However, the lines still contained the CRISPR/Cas9 construct.

Homozygous lines valr4-8, valr6-1, valr1-5-1 and valr1-5-9 were exposed to L. bicolor volatiles as described in chapter 2.1.4. 
Table 13 Components of the T7E1 Assay. The T7 Endonuclease 1 was ordered from New England Biolabs.

\begin{tabular}{|l|c|}
\hline Compound & Volume \\
\hline 10x T7E1 Buffer & $1.1 \mu \mathrm{l}$ \\
\hline T7 Endonuclease $1(10 \mathrm{U} / \mu \mathrm{l})$ & $0.1 \mu \mathrm{l}$ \\
\hline $\mathrm{ddH}_{2} \mathrm{O}$ & $4.8 \mu \mathrm{l}$ \\
\hline
\end{tabular}

\subsection{Test of valr and VALR-OEX lines}

For exposing valr (created with CRISPR/Cas9) and VALR-OEX lines to L. bicolor volatiles, the protocol in chapter 2.1.4 was followed. The mutant seeds and wildtype Col-0 were grown for 4 days before co-cultivation with 14-day-old L. bicolor started (chapter 2.1.3). Co-cultivation was performed for 7 days.

Microscopy of OEX-lines was performed as described in chapter 2.7. 
Table 14. Mutant lines.

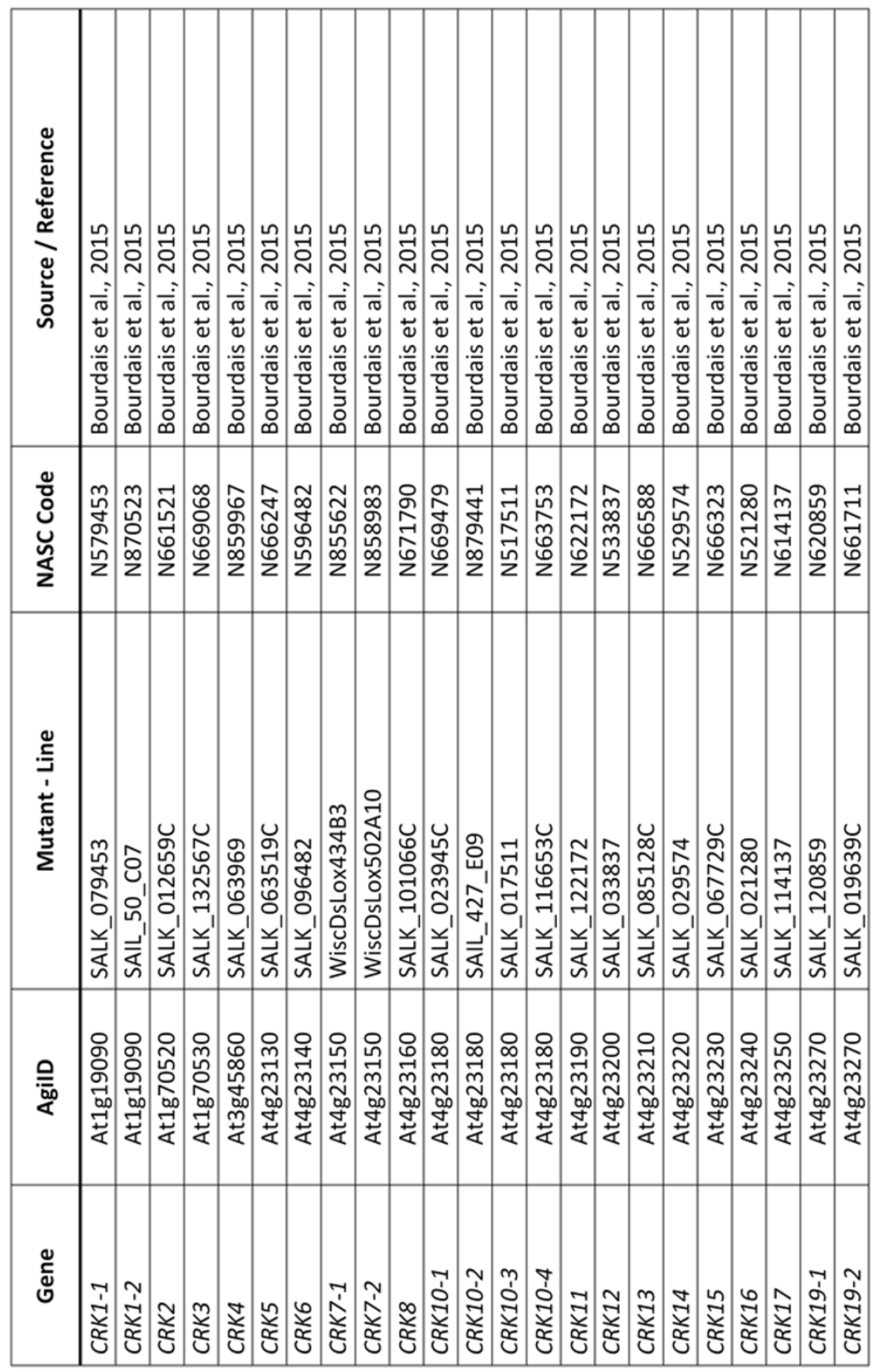




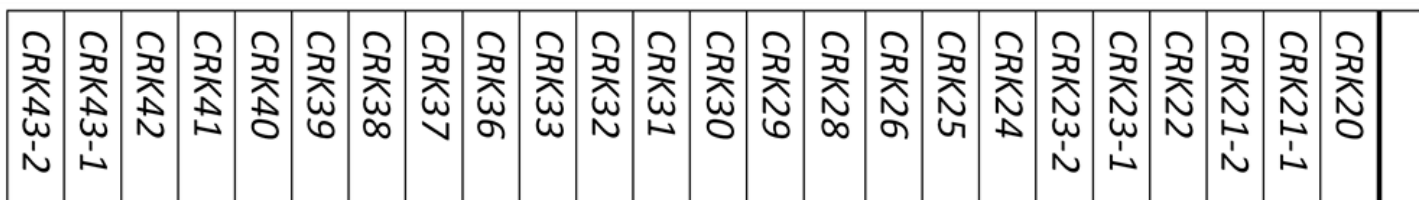

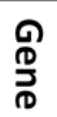

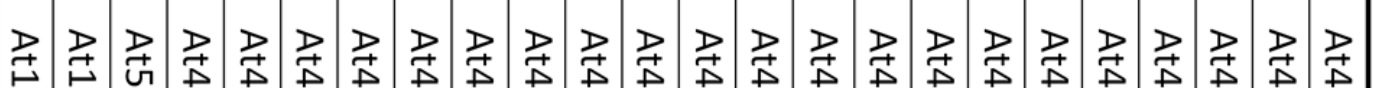

察

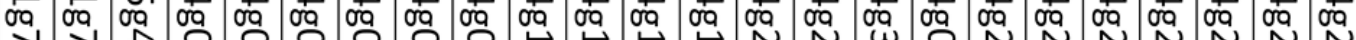

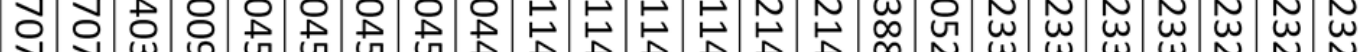

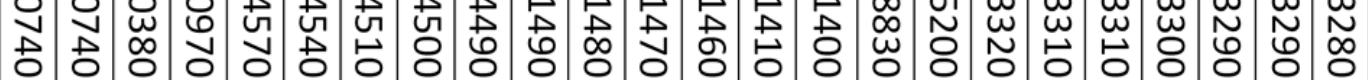

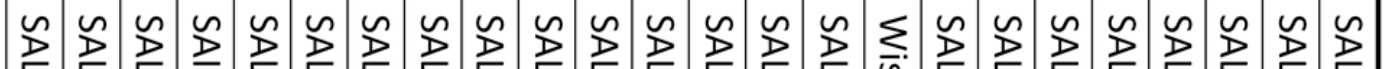

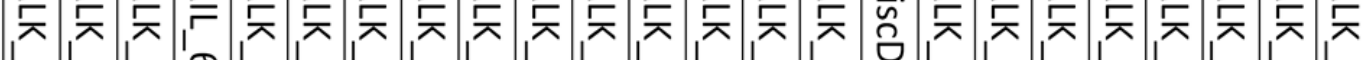
엇요 作

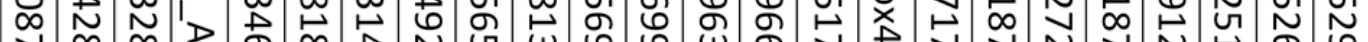

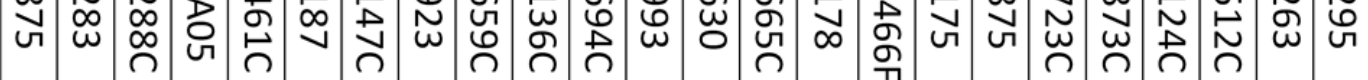
芯

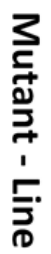

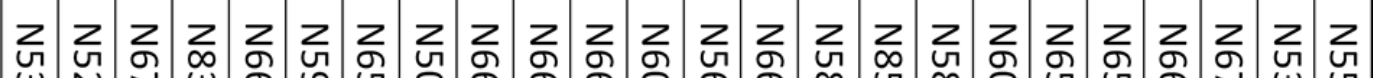

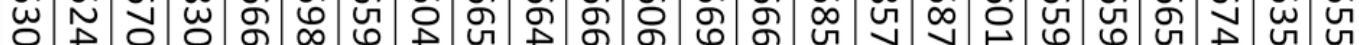

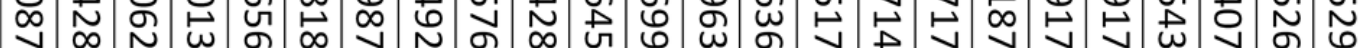

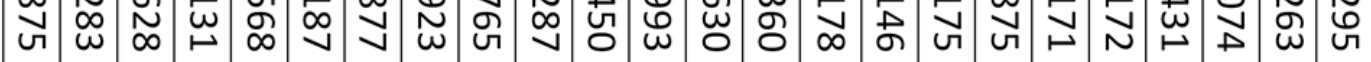

प

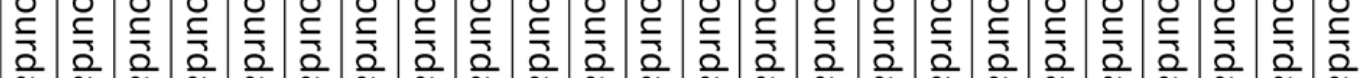

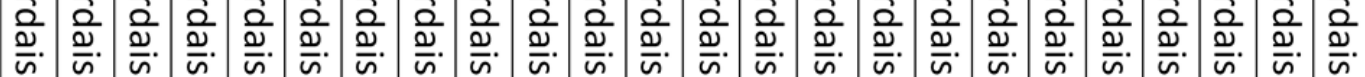

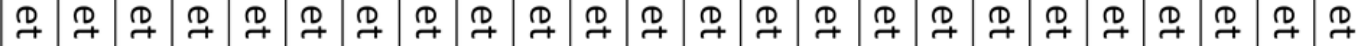

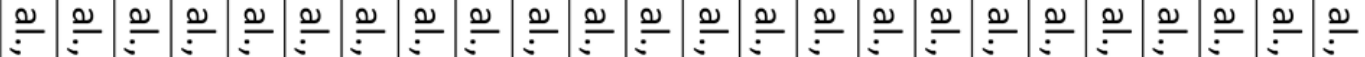
공ำ v 


\begin{tabular}{|c|c|c|c|c|c|c|c|c|c|c|c|c|c|c|c|c|c|c|c|c|c|}
\hline 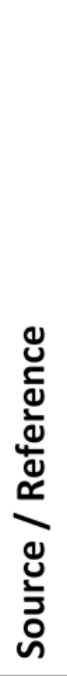 & 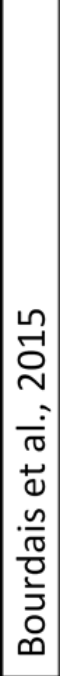 & 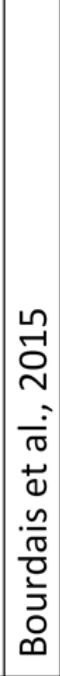 & 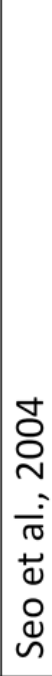 & 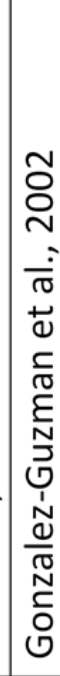 & 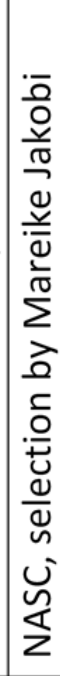 & 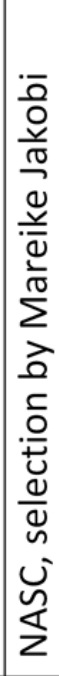 & 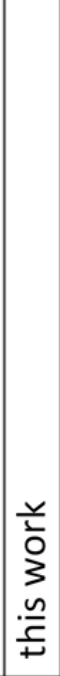 & $\begin{array}{l}\frac{y}{\vdots} \\
\frac{3}{3} \\
\frac{n}{c}\end{array}$ & 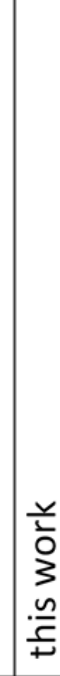 & 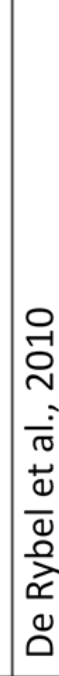 & 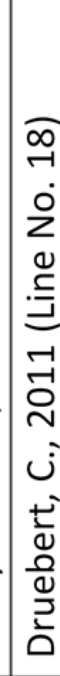 & 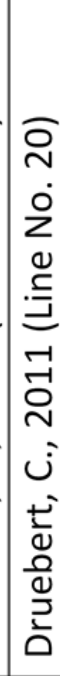 & 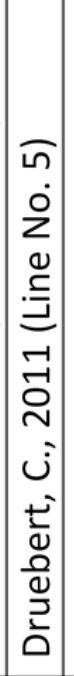 & 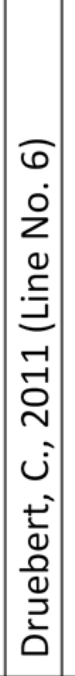 & 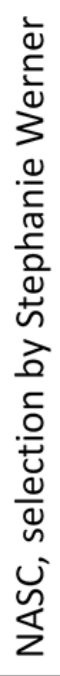 & 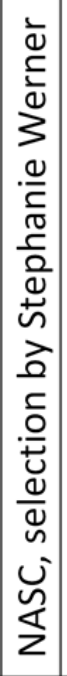 & 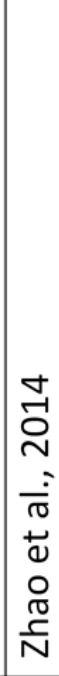 & 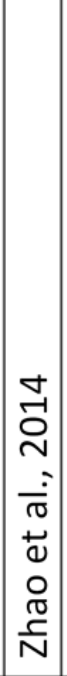 & 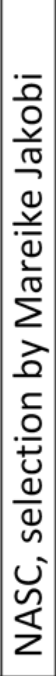 & 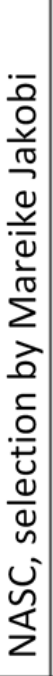 & 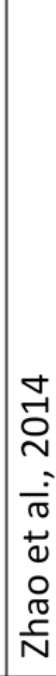 \\
\hline 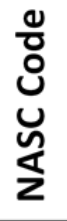 & 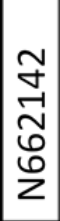 & 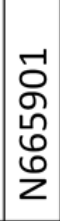 & 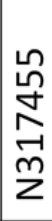 & 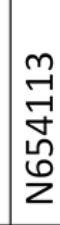 & 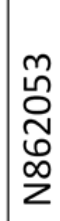 & ' & ' & ' & & ' & ' & I & ' & ' & 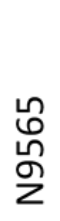 & $\begin{array}{l}\infty \\
0 \\
\text { బू. } \\
2\end{array}$ & , & I & I & 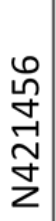 & \\
\hline 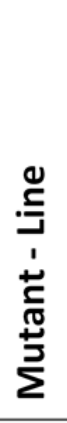 & 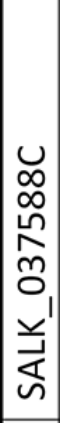 & 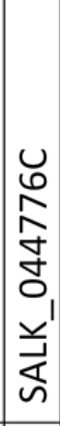 & 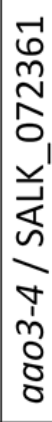 & $\begin{array}{c}1 \\
1 \\
\\
0 \\
0 \\
0 \\
\end{array}$ & 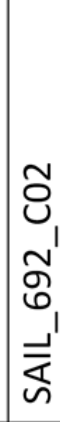 & 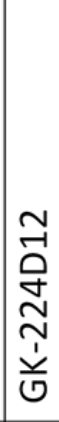 & 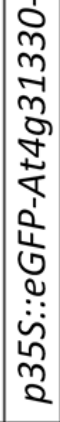 & 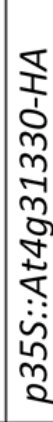 & 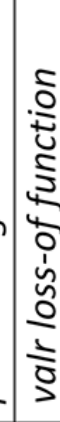 & 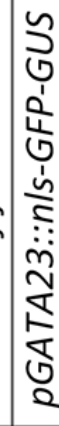 & 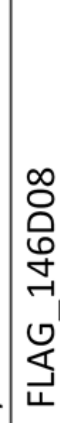 & 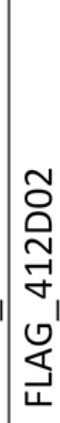 & 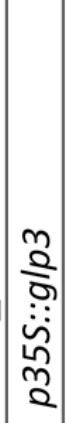 & 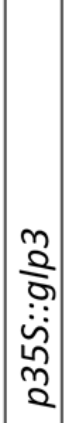 & 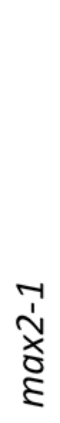 & \begin{tabular}{l}
-1 \\
$\dot{y}$ \\
\multirow{x}{x}{} \\
0 \\
$\varepsilon$
\end{tabular} & 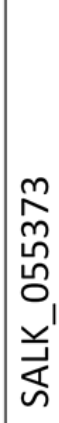 & 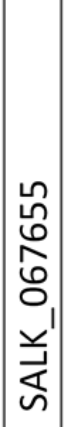 & 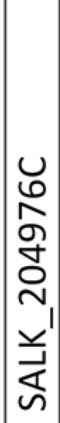 & 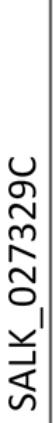 & 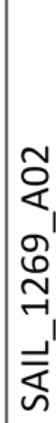 \\
\hline 응 & 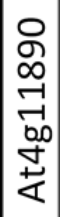 & 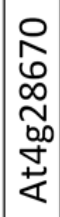 & 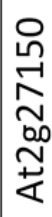 & 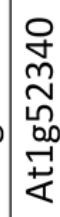 & 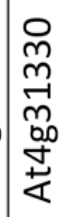 & 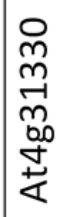 & 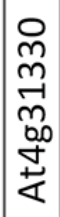 & 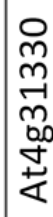 & 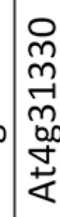 & 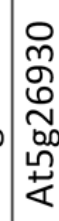 & 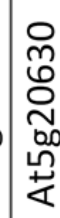 & 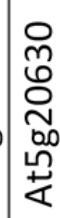 & 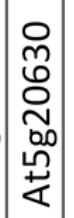 & 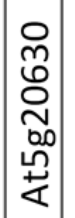 & 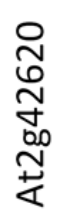 & 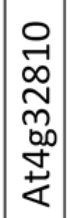 & 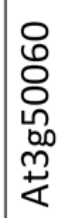 & 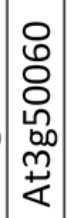 & 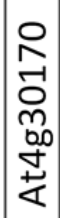 & 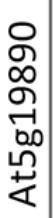 & 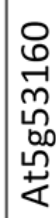 \\
\hline 选 & $\frac{n}{\frac{n}{\sigma}}$ & 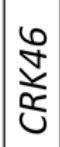 & $\begin{array}{l}0 \\
0 \\
\frac{\pi}{\alpha}\end{array}$ & $\underset{\frac{N}{\alpha}}{\frac{N}{4}}$ & $\stackrel{\frac{\Omega}{3}}{\frac{1}{S}}$ & $\frac{c}{\sqrt{3}}$ & $\frac{a}{3}$ & $\frac{2}{3}$ & $\frac{a}{4}$ & 通 & $\frac{n}{\lesssim}$ & 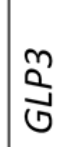 & $\begin{array}{l}m \\
\stackrel{\sim}{\circlearrowleft} \\
\end{array}$ & 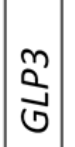 & $\underset{\substack{x \\
\Sigma}}{\stackrel{x}{x}}$ & $\mid \begin{array}{l}\mathbf{z} \\
\mathbf{x} \\
\Sigma\end{array}$ & 今̀ & 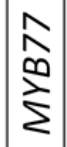 & $\begin{array}{l}n \\
\vdots \\
\frac{2}{w} \\
\alpha\end{array}$ & $\begin{array}{l}\text { जी } \\
\text { of } \\
\frac{1}{2}\end{array}$ & $\stackrel{\infty}{d}$ \\
\hline
\end{tabular}




\begin{tabular}{|c|c|}
\hline 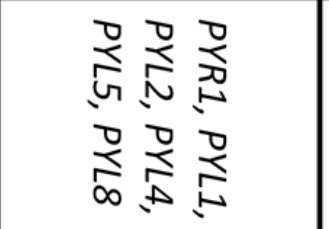 & 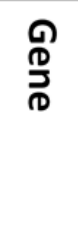 \\
\hline 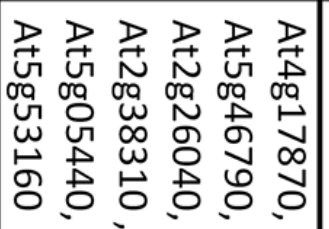 & 离 \\
\hline 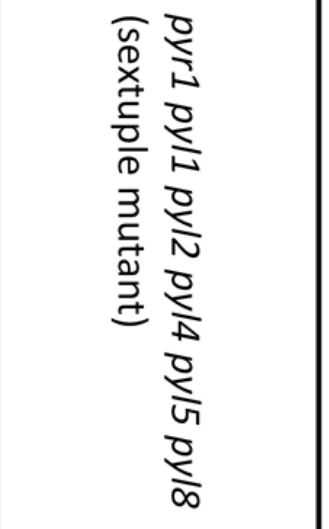 & 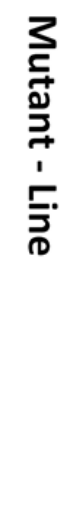 \\
\hline ' & $\begin{array}{l}2 \\
\frac{3}{2} \\
\grave{2} \\
\frac{2}{0}\end{array}$ \\
\hline 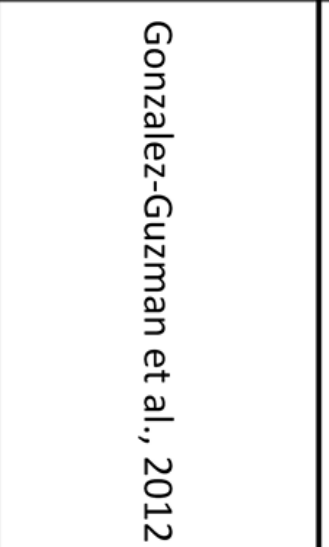 & 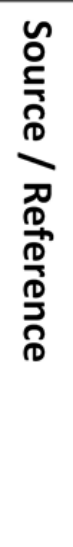 \\
\hline
\end{tabular}


Table 15. Oligonucleotides.

\begin{tabular}{|c|c|c|c|c|c|c|c|c|c|c|c|}
\hline 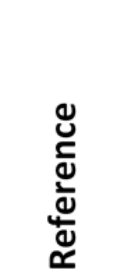 & 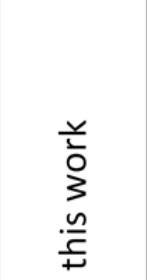 & 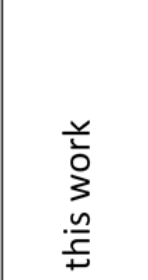 & 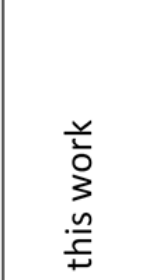 & 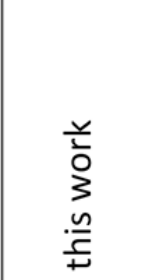 & 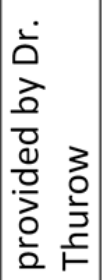 & 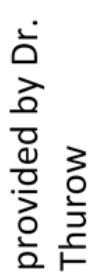 & $\begin{array}{l}\frac{y}{\vdots} \\
\vdots \\
. \frac{n}{c} \\
+\frac{1}{c}\end{array}$ & 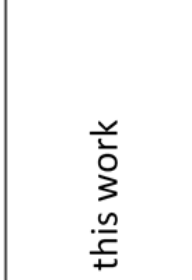 & 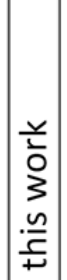 & 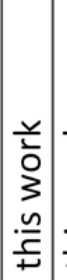 & \\
\hline 토 & $\begin{array}{l}0 \\
\text { in }\end{array}$ & in & 뭉. & $\stackrel{-1}{0}$ & กั & $\begin{array}{l}\text { பn } \\
\text { Uं }\end{array}$ & ஸ̃ & $\begin{array}{l}m \\
8 \\
0\end{array}$ & ñ & นn. & \\
\hline 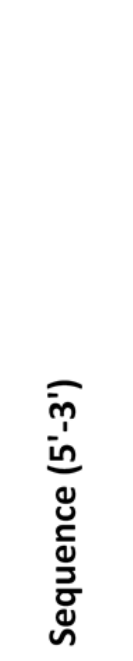 & 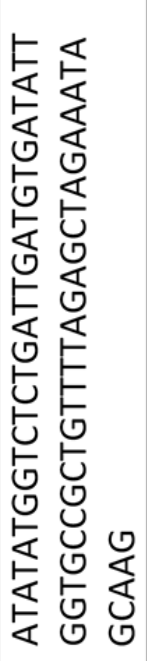 & 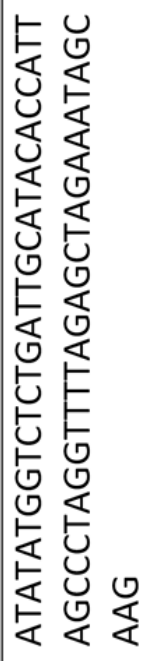 & 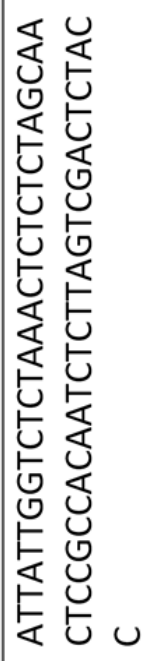 & 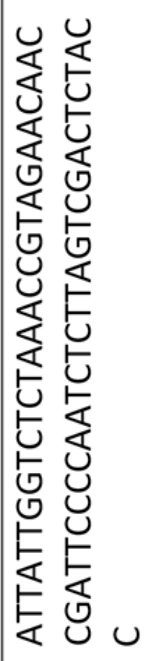 & 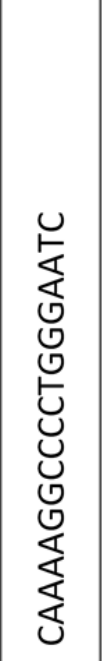 & 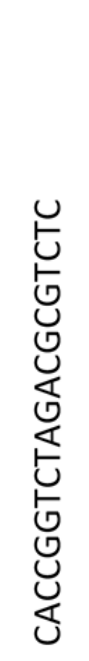 & 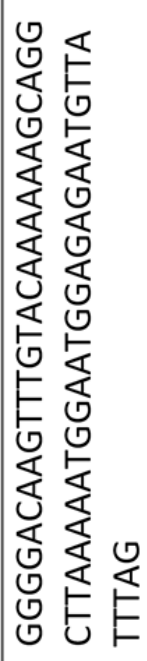 & 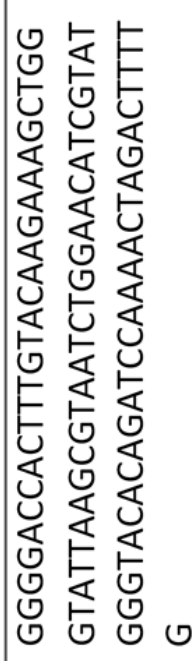 & 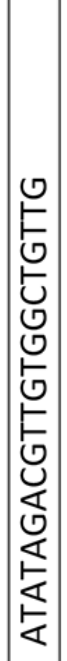 & 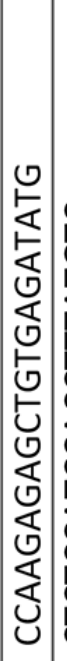 & \\
\hline 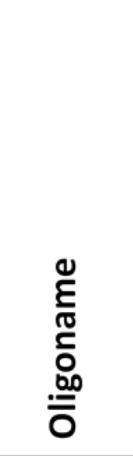 & 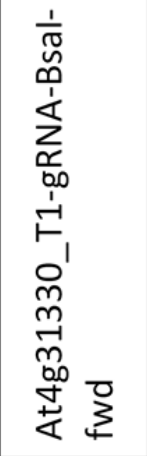 & 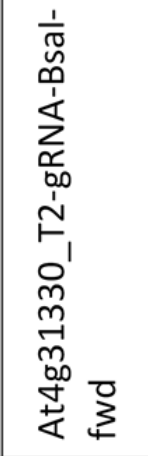 & 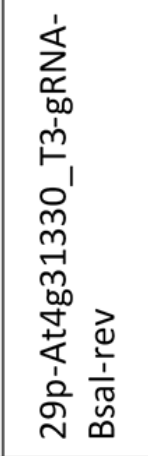 & 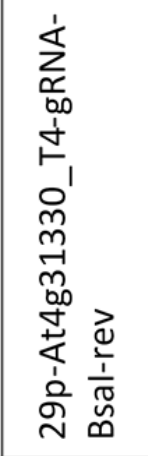 & 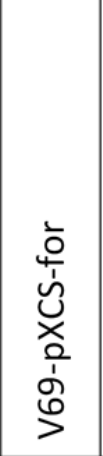 & 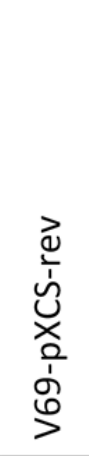 & 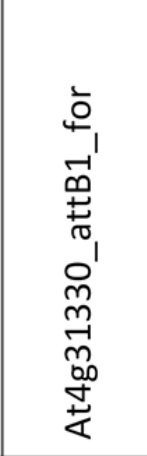 & 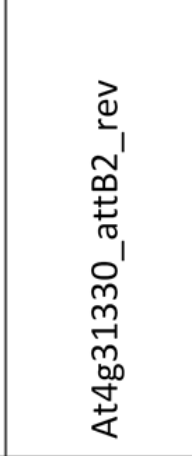 & 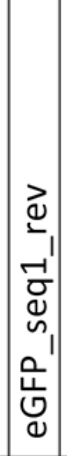 & 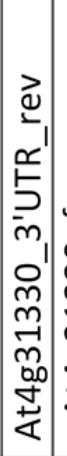 & 芳 \\
\hline 号 & 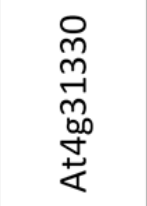 & 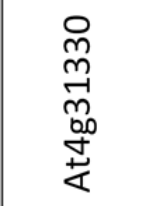 & 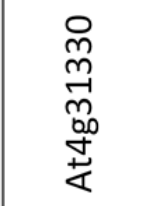 & 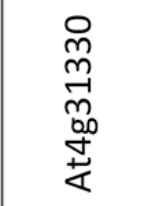 & 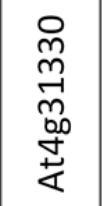 & 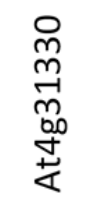 & 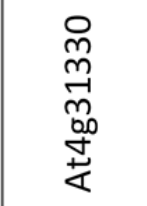 & 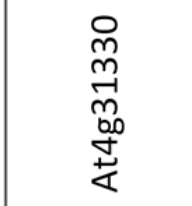 & 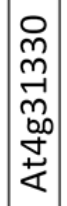 & 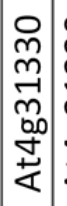 & $\stackrel{b}{+}$ \\
\hline 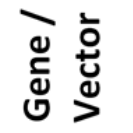 & $\stackrel{s}{s}$ & $\frac{\stackrel{c}{\Delta}}{s}$ & $\frac{\mathfrak{s}}{s}$ & $\frac{\stackrel{c}{d}}{s}$ & $\frac{\mathfrak{s}}{\vec{s}}$ & $\frac{\stackrel{s}{J}}{\vec{s}}$ & $\frac{s}{\frac{\pi}{s}}$ & $\frac{\mathfrak{s}}{s}$ & $\frac{\Omega}{3}$ & $\frac{\infty}{3}$ & \\
\hline 。 & \multicolumn{11}{|c|}{ 8uıगuәnbəs pue 8uluo } \\
\hline
\end{tabular}




\begin{tabular}{|c|c|c|c|c|c|c|c|c|c|c|c|c|c|c|c|c|c|}
\hline \multicolumn{11}{|c|}{ Genotyping } & \multicolumn{6}{|c|}{ Cloning and Sequencing } & \multirow{2}{*}{ 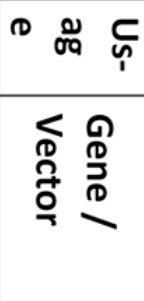 } \\
\hline$\underset{D}{\stackrel{5}{D}}$ & $\underset{\infty}{\frac{\delta}{\infty}}$ & $\underset{\infty}{\stackrel{5}{\infty}}$ & 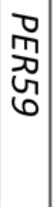 & $\begin{array}{l}7 \\
\text { 勇 } \\
\text { ర }\end{array}$ & $\begin{array}{l}\text { D } \\
\text { 炩 } \\
\text { 心 }\end{array}$ & 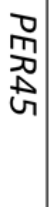 & 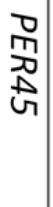 & $\begin{array}{l}\text { D } \\
\text { 而 } \\
\text { जि }\end{array}$ & $\stackrel{\vec{\lambda}}{\stackrel{\lambda}{N}}$ & $\stackrel{\vec{\lambda}}{\stackrel{2}{N}}$ & 另 & 另 & 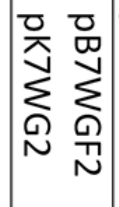 & 읏 畐 & $\begin{array}{l}\text { 믐 } \\
\text { O } \\
\text { 忍 } \\
\text { O }\end{array}$ & $\begin{array}{l}\text { 믐 } \\
\text { O } \\
\text { 忍 } \\
\text { O }\end{array}$ & \\
\hline 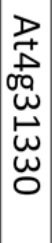 & 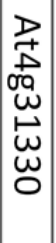 & 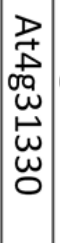 & 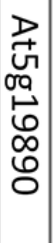 & 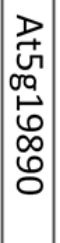 & 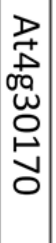 & 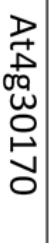 & 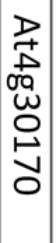 & 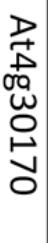 & 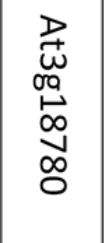 & $\begin{array}{l}\underset{W}{D} \\
\underset{\sigma}{D} \\
\stackrel{\infty}{\infty} \\
\underset{\infty}{D} \\
0\end{array}$ & ' & ' & 1 & ' & ' & ' & $\stackrel{\text { 总 }}{\overline{\text { o }}}$ \\
\hline 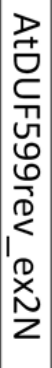 & 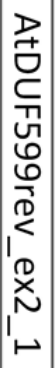 & 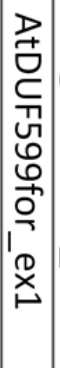 & 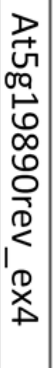 & 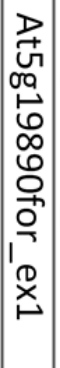 & 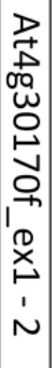 & 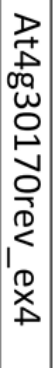 & 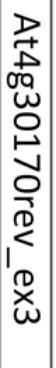 & 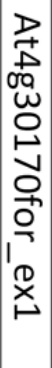 & 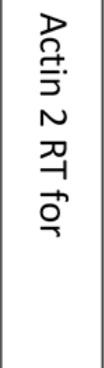 & 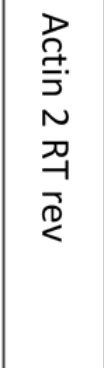 & 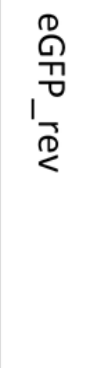 & $\begin{array}{l}\stackrel{D}{0} \\
\text { 赑 } \\
\text { I } \\
\text { 음 }\end{array}$ & 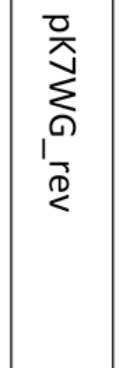 & 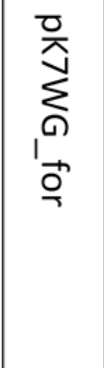 & 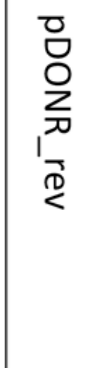 & 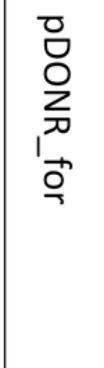 & 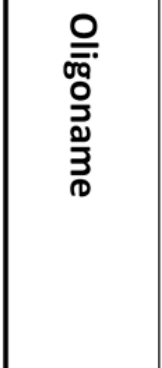 \\
\hline 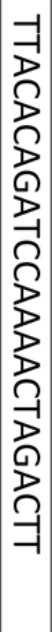 & 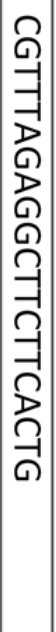 & 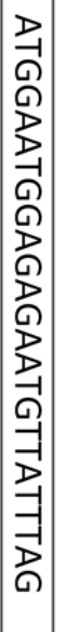 & 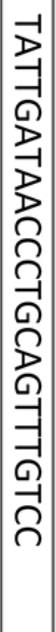 & 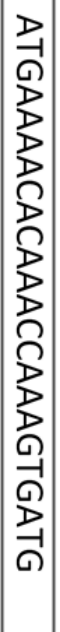 & 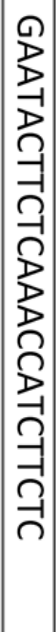 & 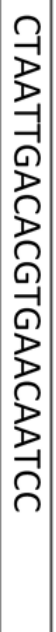 & 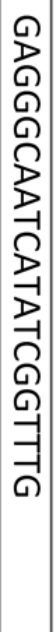 & 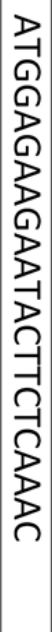 & 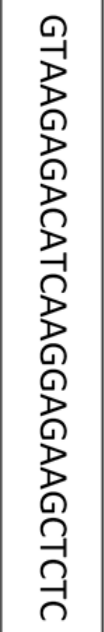 & 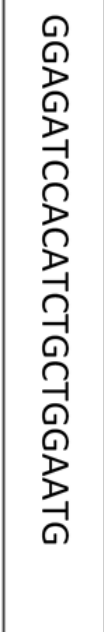 & 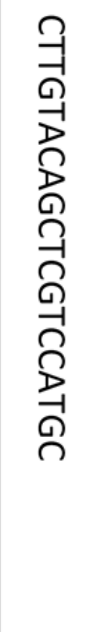 & 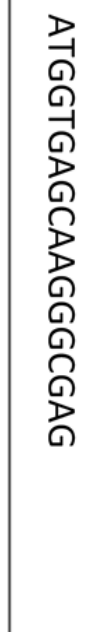 & 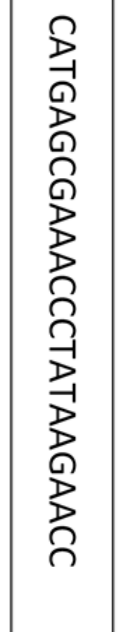 & 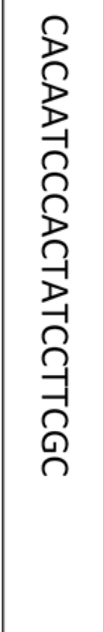 & 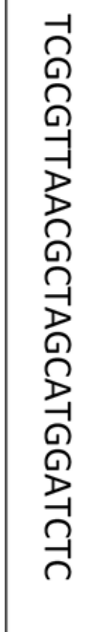 & 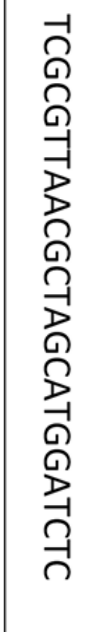 & 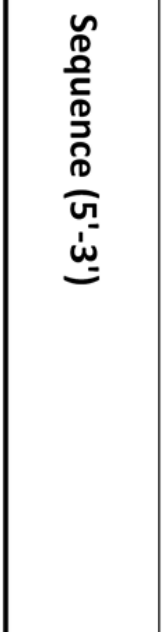 \\
\hline $\begin{array}{l}\mathbf{w} \\
\omega \\
\omega\end{array}$ & $\begin{array}{l}u \\
6 \\
ن\end{array}$ & $\begin{array}{l}\tilde{\omega} \\
\dot{N}\end{array}$ & $\begin{array}{l}\Omega \\
\\
0\end{array}$ & $\left|\begin{array}{c}n \\
\infty \\
\omega\end{array}\right|$ & I & $\begin{array}{l}u \\
\infty \\
\triangle\end{array}$ & $\begin{array}{l}\text { ज़ } \\
\text { vin }\end{array}$ & $\begin{array}{l}\tilde{v} \\
\sigma\end{array}$ & $\begin{array}{l}\text { बे } \\
\stackrel{\sim}{2}\end{array}$ & $\begin{array}{l}\text { के } \\
\dot{\sigma}\end{array}$ & $\begin{array}{l}\text { o } \\
\text { iv }\end{array}$ & $\begin{array}{l}\text { U } \\
\underset{D}{\infty}\end{array}$ & $\begin{array}{l}\stackrel{9}{\omega} \\
\sigma\end{array}$ & $\stackrel{\rho}{\stackrel{\rho}{\sim}}$ & $\underset{i}{g}$ & $\underset{D}{g}$ & กํํ \\
\hline $\begin{array}{l}\text { 空. } \\
\text { L } \\
\text { on } \\
\frac{7}{x}\end{array}$ & $\begin{array}{l}\text { 定. } \\
\bar{n} \\
\sum \\
\text { o } \\
\text { 긋 }\end{array}$ & 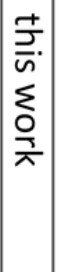 & 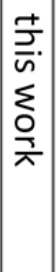 & 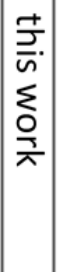 & 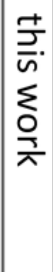 & $\begin{array}{l}\text { 守. } \\
\text { n. } \\
\text { on } \\
\frac{7}{\lambda}\end{array}$ & $\begin{array}{l}\text { 立. } \\
\bar{n} \\
\sum \\
\frac{0}{}\end{array}$ & $\begin{array}{l}\text { 寺. } \\
\text { n. } \\
\sum \\
\text { 읏 }\end{array}$ & 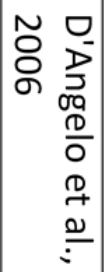 & 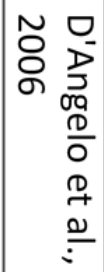 & 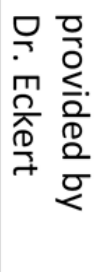 & 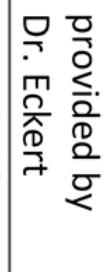 & 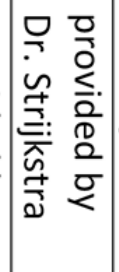 & 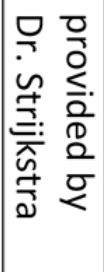 & 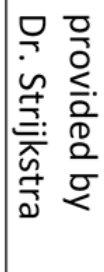 & 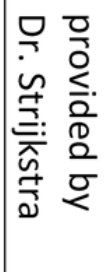 & 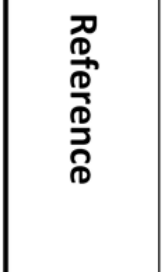 \\
\hline
\end{tabular}




\begin{tabular}{|c|c|c|c|c|c|c|c|c|c|c|c|c|c|c|}
\hline 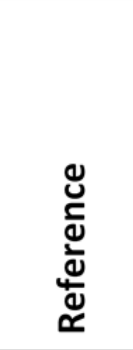 & 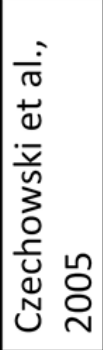 & 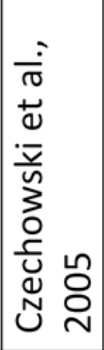 & 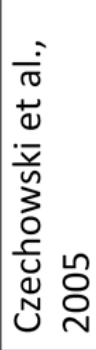 & 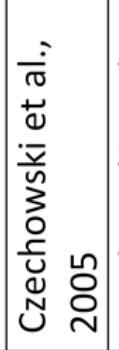 & 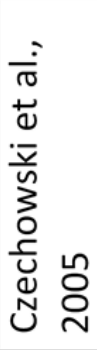 & 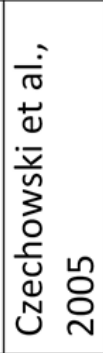 & 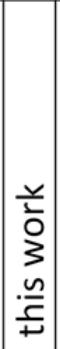 & 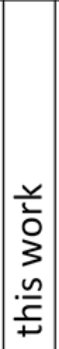 & 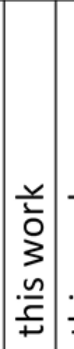 & 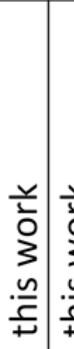 & 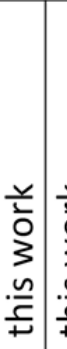 & 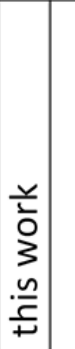 & 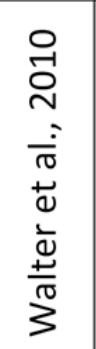 & 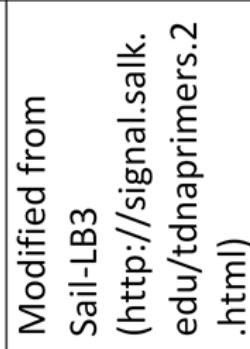 \\
\hline 토 & ભ̣ & $\begin{array}{l}\dot{\sigma} \\
\dot{\sigma}\end{array}$ & $\stackrel{\sim}{\tilde{\sigma}}$ & $\stackrel{\sim}{\tilde{n}}$ & ஸุ & $\stackrel{\nabla}{\infty}$ & $\begin{array}{l}0 \\
\infty \\
\infty \\
n\end{array}$ & $\begin{array}{l}\searrow \\
\infty \\
i n\end{array}$ & $\mid \begin{array}{l}0 \\
\tilde{i} \\
0\end{array}$ & \begin{tabular}{l|l}
\multirow{2}{*}{} & \multicolumn{1}{c}{} \\
$\infty$ &
\end{tabular} & 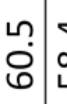 & 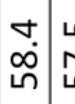 & 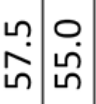 & $\begin{array}{l}m \\
\infty \\
\infty\end{array}$ \\
\hline 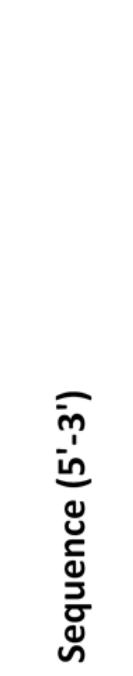 & 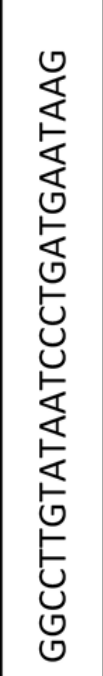 & 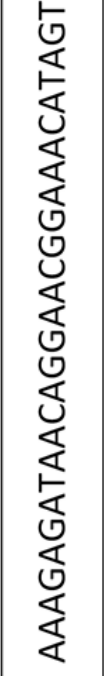 & 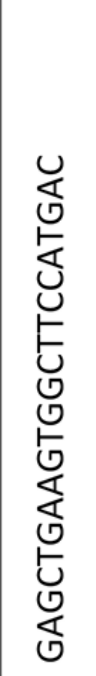 & 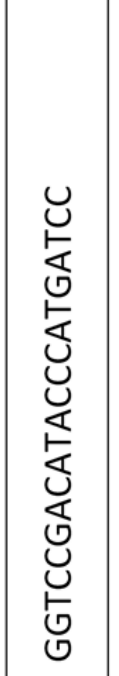 & 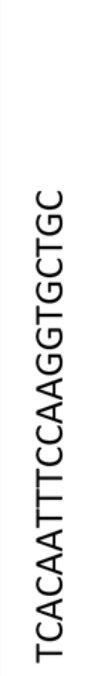 & 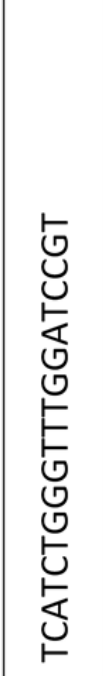 & 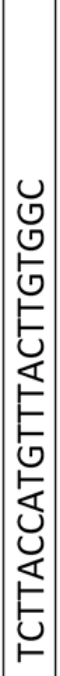 & 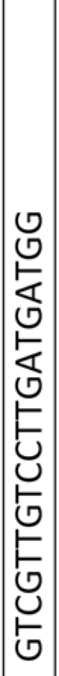 & 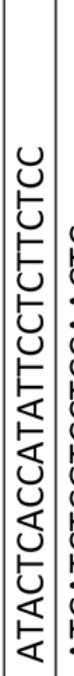 & 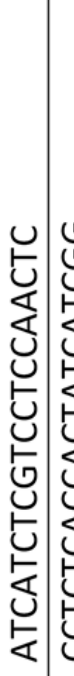 & 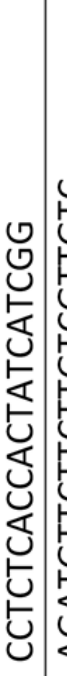 & 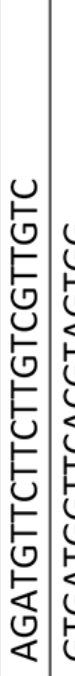 & 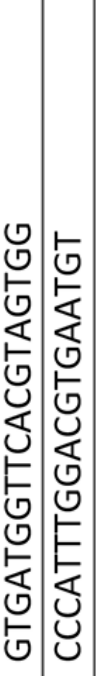 & 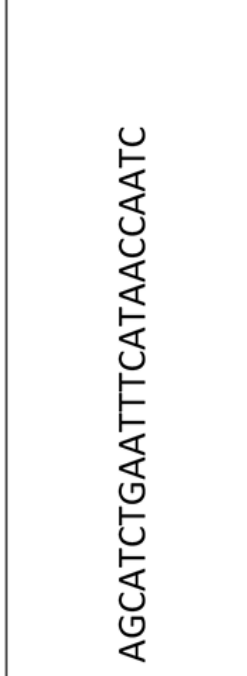 \\
\hline $\begin{array}{l}\stackrel{0}{E} \\
\frac{\pi}{0} \\
\check{0} \\
.00 \\
\overline{0}\end{array}$ & 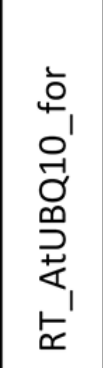 & 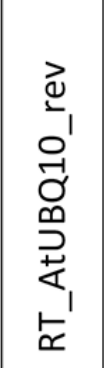 & 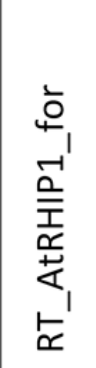 & 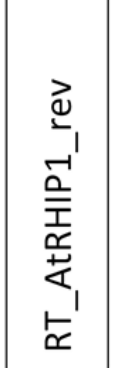 & 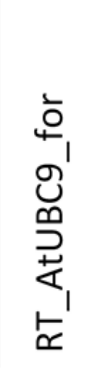 & 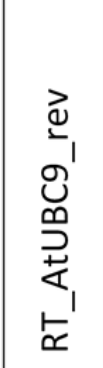 & 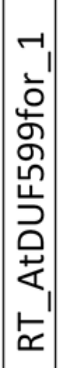 & 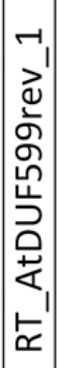 & 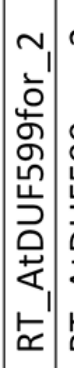 & 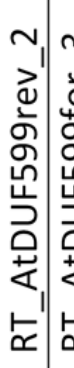 & 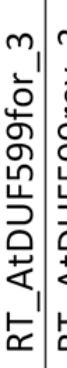 & 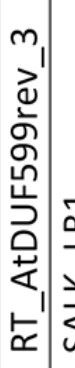 & 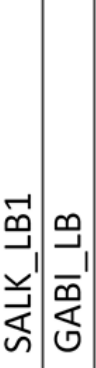 & 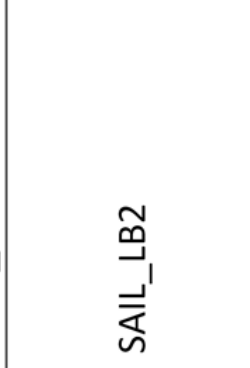 \\
\hline$\stackrel{0}{\stackrel{0}{\alpha 0}}$ & 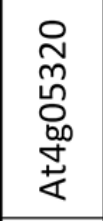 & 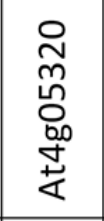 & 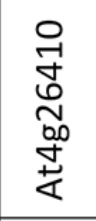 & 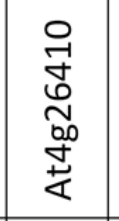 & 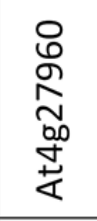 & 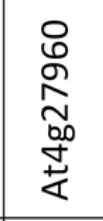 & 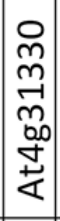 & 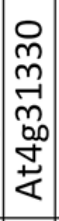 & 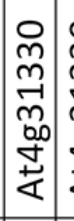 & 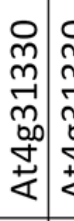 & 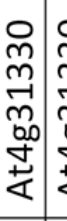 & 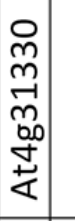 & ' & ' \\
\hline 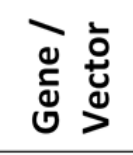 & 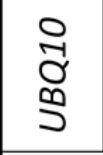 & 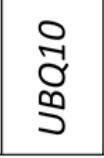 & $\frac{i}{a}$ & $\frac{-1}{\frac{1}{a}}$ & 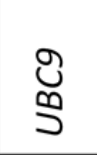 & 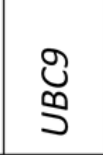 & 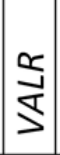 & 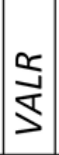 & $\stackrel{s}{2}$ & $\stackrel{5}{\frac{5}{5}}$ & $\stackrel{s}{3}$ & 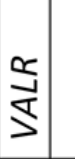 & ' & ' \\
\hline$\stackrel{1}{\lrcorner}$ & \multicolumn{12}{|c|}{ yכd-ıbb } & \multicolumn{2}{|r|}{$\forall N O-\perp$} \\
\hline
\end{tabular}


Table 16. Plasmids used and created in this work.

\begin{tabular}{|c|c|c|c|c|c|c|c|c|c|c|c|}
\hline & 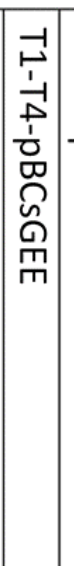 & 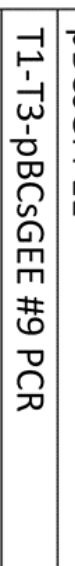 & $\begin{array}{l}0 \\
\tilde{0} \\
\hat{n} \\
\text { ñ } \\
\text { 耑 } \\
\end{array}$ & 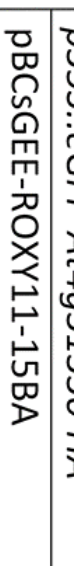 & 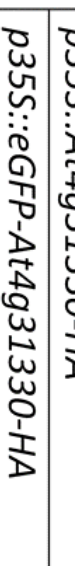 & 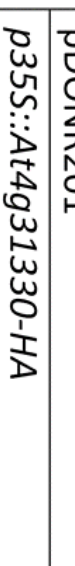 & 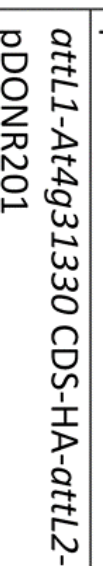 & 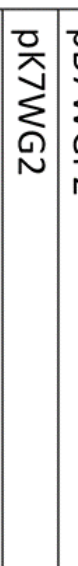 & 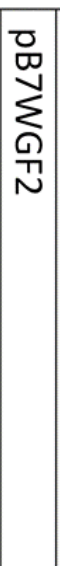 & 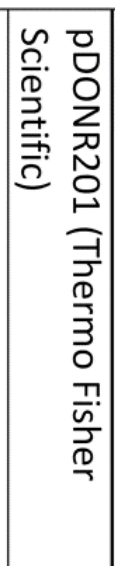 & 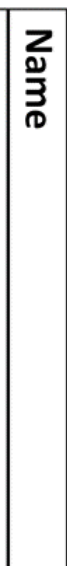 \\
\hline & 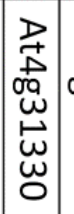 & 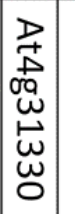 & ' & \begin{tabular}{l}
20 \\
\multirow{2}{*}{} \\
点
\end{tabular} & 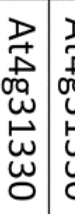 & 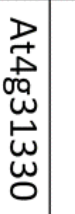 & 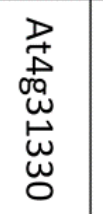 & ' & 1 & & $\begin{array}{l}\stackrel{Q}{\mathbb{D}} \\
\stackrel{\mathbb{D}}{D}\end{array}$ \\
\hline & 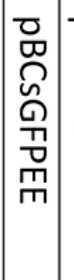 & 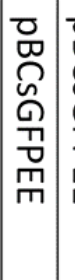 & 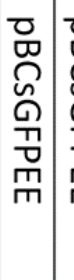 & 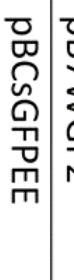 & 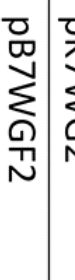 & 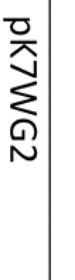 & $\begin{array}{l}\text { ㅁ } \\
\text { O } \\
\text { Z } \\
\text { 공 } \\
\text { ᄋ }\end{array}$ & 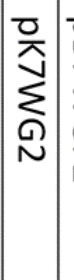 & 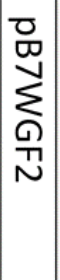 & $\begin{array}{l}\text { ㅁ } \\
\text { O } \\
\sum_{0} \\
\text { 씽 }\end{array}$ & 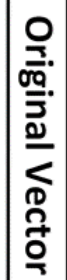 \\
\hline 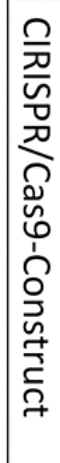 & 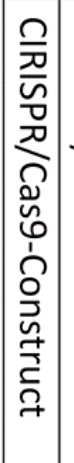 & 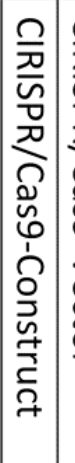 & 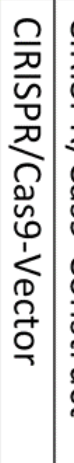 & 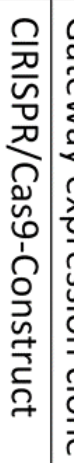 & 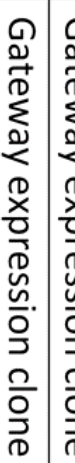 & 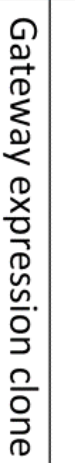 & 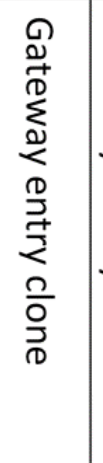 & 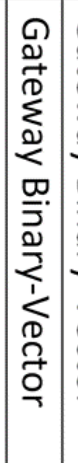 & 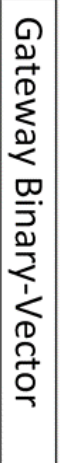 & 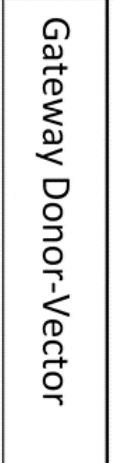 & 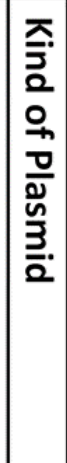 \\
\hline 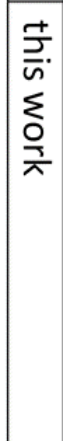 & 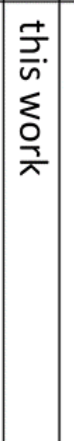 & 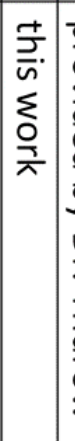 & 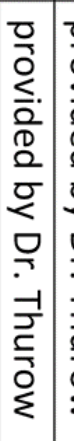 & 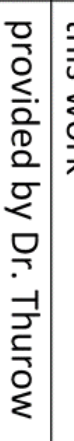 & 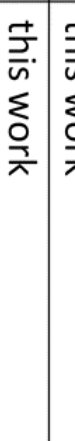 & 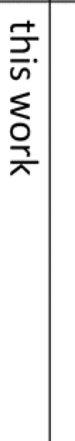 & $\begin{array}{l}\text { 䓂. } \\
\text { n. } \\
\text { 읏 } \\
\text { 읏 }\end{array}$ & 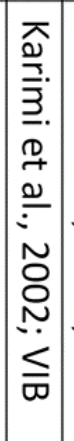 & 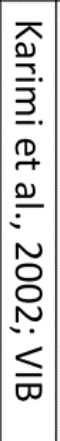 & 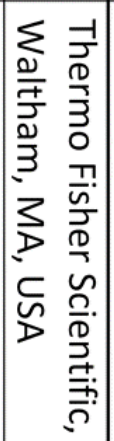 & 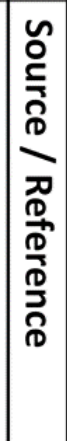 \\
\hline
\end{tabular}




\section{Chemicals}

\begin{tabular}{|c|c|c|}
\hline Chemical & Supplier & Location \\
\hline $\begin{array}{l}\text { 2-(N-morpholino) } \\
\text { ethanesulfonic acid (MES) } \\
\text { sodium salt }\end{array}$ & Carl Roth GmbH \& Co. KG & Karlsruhe, Germany \\
\hline Acetic Acid & Carl Roth GmbH \& Co. KG & Karlsruhe, Germany \\
\hline $\begin{array}{l}\text { Acetonnitril } \\
\text { (LC-MS Chromasolv > 99,9\%) }\end{array}$ & Thermo Fisher Scientific & Waltham, MA, USA \\
\hline Agarose (LE Agarose) & Biozym Scientific GmbH & $\begin{array}{l}\text { Hessisch Oldendorf, } \\
\text { Germany }\end{array}$ \\
\hline Ammonium nitrate $\left(\mathrm{NH}_{4} \mathrm{NO}_{3}\right)$ & Carl Roth GmbH \& Co. KG & Karlsruhe, Germany \\
\hline Bacto Beef Extract & $\begin{array}{l}\text { Becton, Dickinson and } \\
\text { Company }\end{array}$ & $\begin{array}{l}\text { Franklin Lakes, NJ, } \\
\text { USA }\end{array}$ \\
\hline Bacto Peptone & $\begin{array}{l}\text { Becton, Dickinson and } \\
\text { Company }\end{array}$ & $\begin{array}{l}\text { Franklin Lakes, NJ, } \\
\text { USA }\end{array}$ \\
\hline Bacto Tryptone & $\begin{array}{l}\text { Becton, Dickinson and } \\
\text { Company }\end{array}$ & $\begin{array}{l}\text { Franklin Lakes, NJ, } \\
\text { USA }\end{array}$ \\
\hline Bacto Yeast Extract & $\begin{array}{l}\text { Becton, Dickinson and } \\
\text { Company }\end{array}$ & $\begin{array}{l}\text { Franklin Lakes, NJ, } \\
\text { USA }\end{array}$ \\
\hline Boric acid (H3BO3) & Carl Roth GmbH \& Co. KG & Karlsruhe, Germany \\
\hline $\begin{array}{l}\text { Calcium chloride dihydrate } \\
\left(\mathrm{CaCl}_{2} \times 2 \mathrm{H}_{2} \mathrm{O}\right)\end{array}$ & Carl Roth GmbH \& Co. KG & Karlsruhe, Germany \\
\hline \multicolumn{3}{|l|}{ Camphorsulfonic acid } \\
\hline Chloroform & Carl Roth GmbH \& Co. KG & Karlsruhe, Germany \\
\hline $\begin{array}{l}\text { Cobalt (II) chloride } \\
\text { hexahydrate }\left(\mathrm{CoCl}_{2} \times 6 \mathrm{H}_{2} \mathrm{O}\right)\end{array}$ & Merck & Darmstadt, Germany \\
\hline $\begin{array}{l}\text { Copper sulfate pentahydrate } \\
\left(\mathrm{CuSO}_{4} \times 5 \mathrm{H}_{2} \mathrm{O}\right)\end{array}$ & Merck & Darmstadt, Germany \\
\hline $\begin{array}{l}\text { CTAB } \\
\text { (Cetyltrimethylammonium } \\
\text { bromide) }\end{array}$ & Carl Roth GmbH \&Co. KG & Karlsruhe, Germany \\
\hline $\begin{array}{l}\text { Di-ammonium tartrate } \\
\left(\mathrm{C}_{4} \mathrm{H}_{12} \mathrm{~N}_{2} \mathrm{O}_{6}\right)\end{array}$ & Merck & Darmstadt, Germany \\
\hline $\begin{array}{l}\text { EDTA } \\
\text { (Ethylenediaminetetraacetic } \\
\text { acid) }\end{array}$ & Carl Roth GmbH \&Co. KG & Karlsruhe, Germany \\
\hline Ethanol $96 \%$ & Carl Roth GmbH \&Co. KG & Karlsruhe, Germany \\
\hline $\begin{array}{l}\text { Farnesyl pyrophosphate } \\
\text { ammonium salt }\end{array}$ & Sigma-Aldrich & St. Louis, MO, USA \\
\hline $\begin{array}{l}\text { Formic acid (Eluent additive } \\
\text { for } L C M S \text { ) }\end{array}$ & Thermo Fisher Scientific & Waltham, MA, USA \\
\hline Gelrite & Duchefa Biochemie B.V. & Haarlem, Netherlands \\
\hline Glucose & Carl Roth GmbH \& Co. KG & Karlsruhe, Germany \\
\hline
\end{tabular}




\begin{tabular}{|c|c|c|}
\hline $\begin{array}{l}\text { Glufosinate-ammonium } \\
\text { (Pestanal) }\end{array}$ & Sigma-Aldrich & St. Louis, MO, USA \\
\hline Glycerin & Carl Roth GmbH \& Co. KG & Karlsruhe, Germany \\
\hline Glycine & $\begin{array}{l}\text { Serva Electrophoresis } \\
\text { GmbH }\end{array}$ & Heidelberg, Germany \\
\hline Isoamylalcohol & Merck & Darmstadt, Germany \\
\hline Isopropanol (2-Propanol) & Carl Roth GmbH \&Co. KG & Karlsruhe, Germany \\
\hline $\begin{array}{l}\text { Isopropanol (2-Propanol); } \\
\text { Rotisolv HPLC }\end{array}$ & Carl Roth GmbH \& Co. KG & Karlsruhe, Germany \\
\hline $\begin{array}{l}\text { Kanieltra microelement } \\
\text { solution }\end{array}$ & Yara & Nanterra, France \\
\hline Lithium chloride & Carl Roth GmbH \& Co. KG & Karlsruhe, Germany \\
\hline $\begin{array}{l}\text { Magnesium sulfate } \\
\text { heptahydrate }\left(\mathrm{MgSO}_{4} \times 7\right. \\
\left.\mathrm{H}_{2} \mathrm{O}\right)\end{array}$ & Carl Roth GmbH \& Co. KG & Karlsruhe, Germany \\
\hline Maltose & Carl Roth GmbH \& Co. KG & Karlsruhe, Germany \\
\hline $\begin{array}{l}\text { Manganese (II) sulfate } \\
\text { monohydrate }\left(\mathrm{MnSO}_{4} \times \mathrm{H}_{2} \mathrm{O}\right)\end{array}$ & Merck & Darmstadt, Germany \\
\hline $\begin{array}{l}\text { Methanol (hypergrade for } \\
\text { LC-MS) }\end{array}$ & Merck & Darmstadt, Germany \\
\hline Micro Agar & Duchefa Biochemie B.V. & Haarlem, Netherlands \\
\hline $\begin{array}{l}\text { MS (Murashige and Skoog) } \\
\text { salt }\end{array}$ & Duchefa Biochemie B.V. & Haarlem, Netherlands \\
\hline Myo-Inositol & Duchefa Biochemie B.V. & Haarlem, Netherlands \\
\hline Natriumhypochlorit (2.8\%) & DanKlorix, CP GABA GmbH & Hamburg, Germany \\
\hline Niacin & Merck & Darmstadt, Germany \\
\hline \multicolumn{3}{|l|}{ n-Nitrophenol sodium salt } \\
\hline Phyto Agar & Duchefa Biochemie B.V. & Haarlem, Netherlands \\
\hline $\begin{array}{l}\text { Potassium dihydrogen } \\
\text { phosphate }\left(\mathrm{KH}_{2} \mathrm{PO}_{4}\right)\end{array}$ & Carl Roth GmbH \& Co. KG & Karlsruhe, Germany \\
\hline Potassium hydroxide $(\mathrm{KOH})$ & Merck & Darmstadt, Germany \\
\hline Potassium iodide (KI) & Merck & Darmstadt, Germany \\
\hline Potassium nitrate $\left(\mathrm{KNO}_{3}\right)$ & Carl Roth GmbH \& Co. KG & Karlsruhe, Germany \\
\hline $\begin{array}{l}\text { PVP (K30; } \\
\text { Polyvinylpyrrolidon) }\end{array}$ & Sigma-Aldrich & St. Louis, MO, USA \\
\hline Pyridoxine $\mathrm{HCl}$ & Sigma-Aldrich & St. Louis, MO, USA \\
\hline Saccharose & Carl Roth GmbH \&Co. KG & Karlsruhe, Germany \\
\hline Saccharose & Carl Roth GmbH \& Co. KG & Karlsruhe, Germany \\
\hline SDS (Sodium dodecyl sulfate) & Carl Roth GmbH \&Co. KG & Karlsruhe, Germany \\
\hline Silwet L-77 (Vac-in-Stuff) & Lehle Seeds & Round Rock, TX, USA \\
\hline Sodium chloride $(\mathrm{NaCl})$ & Carl Roth GmbH \&Co. KG & Karlsruhe, Germany \\
\hline $\begin{array}{l}\text { Sodium feredetate } \\
\left(\mathrm{C}_{10} \mathrm{H}_{12} \mathrm{FeN}_{2} \mathrm{NaO}_{8}\right)\end{array}$ & Carl Roth GmbH \& Co. KG & Karlsruhe, Germany \\
\hline $\begin{array}{l}\text { Sodium molybdate dihydrate } \\
\left(\mathrm{Na}_{2} \mathrm{MoO}_{4} \times 2 \mathrm{H}_{2} \mathrm{O}\right)\end{array}$ & Merck & Darmstadt, Germany \\
\hline
\end{tabular}




\begin{tabular}{|l|l|l|}
\hline Thiamine $\mathrm{HCl}$ & Merck & Darmstadt, Germany \\
\hline Tris-Base & Carl Roth GmbH \& Co. KG & Karlsruhe, Germany \\
\hline Tris- $\mathrm{HCl}$ & Carl Roth GmbH \&Co. KG & Karlsruhe, Germany \\
\hline Triton ${ }^{\circledR}$ X-100 & $\begin{array}{l}\text { Serva Electrophoresis } \\
\text { GmbH }\end{array}$ & Heidelberg, Germany \\
\hline Water LC-MS grade & Merck & Darmstadt, Germany \\
\hline $\begin{array}{l}\text { Water molecular biology } \\
\text { grade }\end{array}$ & AppliChem GmbH & Darmstadt, Germany \\
\hline $\begin{array}{l}\text { Zinc sulfate heptahydrate } \\
\left(\mathrm{ZnSO}_{4}+7 \mathrm{H}_{2} \mathrm{O}\right)\end{array}$ & Merck & Darmstadt, Germany \\
\hline
\end{tabular}

\section{Antibiotics}

\begin{tabular}{|l|l|l|}
\hline Antibiotic & Supplier & Location \\
\hline $\begin{array}{l}\text { Kanamycin Sulphate } \\
\text { Monohydrate }\end{array}$ & Duchefa Biochemie B.V. & Haarlem, Netherlands \\
\hline Rifampicin & Duchefa Biochemie B.V. & Haarlem, Netherlands \\
\hline $\begin{array}{l}\text { Gentamycin Sulphate } \\
\text { Spectinomycin } \\
\text { Dihydrochloride 5-hydrate }\end{array}$ & Duchefa Biochemie B.V. & Haarlem, Netherlands \\
\hline
\end{tabular}




\section{Enzymes}

\begin{tabular}{|l|l|l|}
\hline Enzyme & Supplier & Location \\
\hline Bsal-HFv2 & New England Biolabs & Ipswich, MA, USA \\
\hline $\begin{array}{l}\text { Gateway BP Clonase II Enzyme } \\
\text { Gateway LR Clonase II Enzyme } \\
\text { mix }\end{array}$ & Invitrogen & Carlsbad, CA, USA \\
\hline $\begin{array}{l}\text { Phusion High Fidelity DNA } \\
\text { Polyermase }\end{array}$ & Thermo Fisher Scientific & Waltham, MA, \\
\hline T4 Ligase & New England Biolabs & Ipswich, MA, USA \\
\hline T7 Endonuclease 1 & New England Biolabs & Ipswich, MA, USA \\
\hline Taq DNA Polymerase & Thermo Fisher Scientific & $\begin{array}{l}\text { Waltham, MA, } \\
\text { USA }\end{array}$ \\
\hline
\end{tabular}




\section{Kits}

\begin{tabular}{|l|l|l|}
\hline Kit & Supplier & Location \\
\hline $\begin{array}{l}\text { innuMIX qPCR DSGreen } \\
\text { Standard }\end{array}$ & analytik jena AG & Jena, Germany \\
\hline innuPREP PCRpure Kit & analytik jena AG & Jena, Germany \\
\hline innuPREP Plant RNA Kit & analytik jena AG & Jena, Germany \\
\hline innuPREP Plasmid Mini Kit & analytik jena AG & Jena, Germany \\
\hline $\begin{array}{l}\text { NEBNext Poly (A) mRNA } \\
\text { Magnetic Isolation } \\
\text { Module }\end{array}$ & New England Biolabs & Ipswich, MA, USA \\
\hline $\begin{array}{l}\text { NEBNext Ultra RNA Library } \\
\text { Prep Kit for Illumina }\end{array}$ & New England Biolabs & Ipswich, MA, USA \\
\hline $\begin{array}{l}\text { RevertAid First Strand } \\
\text { cDNA Synthesis Kit }\end{array}$ & Thermo Fisher Scientific & Waltham, MA, USA \\
\hline RNA Nano Chip Kit & Agilent & Santa Clara, CA, USA \\
\hline RNase-Free DNase Set & Qiagen GmbH & Hilden, Germany \\
\hline RNeasy Plant Mini Kit & Qiagen GmbH & \\
\hline
\end{tabular}




\section{Devices}

\begin{tabular}{|c|c|c|c|}
\hline Device & Model & Supplier & Location \\
\hline Bioanalyzer & $\begin{array}{l}\text { Agilent } \\
\text { Bioanalyzer } 2100\end{array}$ & Agilent & Santa Clara, CA, USA \\
\hline Centrifuge & $5427 R$ & Eppendorf AG & Hamburg, Germany \\
\hline Centrifuge & $5417 R$ & Eppendorf AG & Hamburg, Germany \\
\hline Centrifuge & $\mathrm{J} 2-\mathrm{HS}$ & Beckman Coulter & Brea, CA, USA \\
\hline Climate Chamber & AR 75-L & Percival & Perry, IA, USA \\
\hline Electroporator & MicroPulse & $\begin{array}{l}\text { BioRad Laboratories } \\
\text { Inc. }\end{array}$ & Hercules, CA, USA \\
\hline \multicolumn{4}{|c|}{ Electrospray Ionisation (ESI) source } \\
\hline Gel chamber & $\begin{array}{l}\text { Mini-Sub Cell GT } \\
\text { Cell systems }\end{array}$ & $\begin{array}{l}\text { BioRad Laboratories } \\
\text { Inc. }\end{array}$ & Hercules, CA, USA \\
\hline Gel imager & Gel IX Imager & intas & Göttingen, Germany \\
\hline $\begin{array}{l}\text { Impact II UHR- } \\
\text { QqTOF }\end{array}$ & & $\begin{array}{ll}\text { Bruker } & \text { Daltonic } \\
\text { GmbH } & \end{array}$ & Bremen, Germany \\
\hline LED bulbs & $\begin{array}{l}\text { MM21091-LED } \\
\text { Classic, 7.4 Watt }\end{array}$ & Megaman & $\begin{array}{l}\text { Langenselbold, } \\
\text { Germany }\end{array}$ \\
\hline Microscope & $\begin{array}{l}\text { Axioplan } \\
\text { Observer.Z1 }\end{array}$ & Carl Zeiss GmbH & $\begin{array}{l}\text { Oberkochen, } \\
\text { Germany }\end{array}$ \\
\hline NanoDrop & NanoDrop 2000 & $\begin{array}{l}\text { Thermo Fisher } \\
\text { Scientific }\end{array}$ & Waltham, MA, USA \\
\hline Neon Tubes & $\begin{array}{l}\text { F32T8/TL741, } 32 \\
\text { Watt }\end{array}$ & Philips & $\begin{array}{l}\text { Amsterdam, } \\
\text { Netherlands }\end{array}$ \\
\hline Thermocycler & $\begin{array}{l}\text { Labcycler } \\
\text { Gradient }\end{array}$ & Sensoquest & Göttingen, Germany \\
\hline Photometer & $\begin{array}{l}\text { BioPhotometer } \\
6131\end{array}$ & Eppendorf AG & Hamburg, Germany \\
\hline Power Supply & PowerPac 200 & $\begin{array}{l}\text { BioRad Laboratories } \\
\text { Inc. }\end{array}$ & Hercules, CA, USA \\
\hline
\end{tabular}


Material and Methods

\begin{tabular}{|l|l|l|l|}
\hline qRT-PCR cycler & q Tower $^{3}$ G & analytik jena AG & Jena, Germany \\
\hline RNAseq & $\begin{array}{l}\text { NextSeq500 } \\
\text { instrument }\end{array}$ & Illumina & San Diego, CA, USA \\
\hline Speedvac & $\begin{array}{l}\text { Concentrator } \\
5301\end{array}$ & Eppendorf AG & Hamburg, Germany \\
\hline Stereomicroscope & M205FA & $\begin{array}{l}\text { Leica Microsystems } \\
\text { GmbH }\end{array}$ & Wetzlar, Germany \\
\hline Stereomicroscope & M205FCA & $\begin{array}{l}\text { Leica Microsystems } \\
\text { GmbH }\end{array}$ & Wetzlar, Germany \\
\hline Swing Mill & MM400 & Retsch GmbH & Haan, Germany \\
\hline UltiMate 3000RS & & Thermo & Fisher \\
\hline Ultrasonic Bath & $\begin{array}{l}\text { Sonorex Super } \\
\text { 10P }\end{array}$ & $\begin{array}{l}\text { BANDELIN electronic } \\
\text { GmbH \& Co. KG }\end{array}$ & Berlin, Germany \\
\hline
\end{tabular}




\section{Results}

\subsection{Impact of experimental conditions on the response to volatiles of L. bicolor in A. thaliana roots}

\subsubsection{Effect of L. bicolor age on LR formation in A. thaliana}

Since the intensity of the response of root density to volatile exposure was variable among different experiments, we investigated the influence of fungal age on the increase in root densities. After 9 or 18 days of preculture, L. bicolor grown in aluminum trays was added to 4-day-old $A$. thaliana plants.

After co-cultivation for 7 days, all seedlings, no matter if they were exposed to volatiles of 9-day-old or 18-day-old fungi, showed enhanced LR density compared to control conditions but the effect was slightly stronger (1.2-fold) for 9 day-old than for 18 day-old (1.1-fold) fungi (Figure 12A). Because 9-day-old plugs of L. bicolor developed rather little mycelium, we decided to use 11- to 14-day-old L. bicolor plugs for the following experiments.

\subsubsection{Difference between aluminum trays or cellophane foil for L. bicolor growth on}

\section{A. thaliana development}

This investigation focused on the growth conditions of $L$. bicolor. When the fungus was grown on P20 medium in aluminum trays without cellophane foil, we noticed growth inside the medium. This might have an adverse effect on the emission of volatiles of L. bicolor. Furthermore, aluminum might affect plant growth as well (Mossor-Pietraszewska, 2001; Panda et al., 2009). Therefore, we placed four fungus plugs on cellophane membranes on P20 medium and cultivated the fungus for 14 days (as described in chapter 2.2.2). For co-cultivation the fungus on the cellophane membrane was transferred to the bi-compartmented petri dish on the P20 medium, similar as it was performed by Ditengou et al., 2015. Besides, L. bicolor was grown on P20 medium in aluminum trays.

After co-cultivation, petri dishes were analyzed visually (Figure 12B). The increase in LR density in volatile exposed plants in comparison to control plants was observed regardless of the fungal growth condition. However, the difference between treated and 
non-treated plants was more obvious with $A$. thaliana roots co-cultivated with the fungus grown on a cellophane membrane (Figure 12B). Consequently, cellophane membranes were used for following experiments.

\subsubsection{Comparison of $A$. thaliana growth in Phyto agar and Gelrite at different pH values}

Growth media have a strong influence on root architecture. Initially, we used Phytoagar to solidify AM medium. However, Ditengou et al., 2015 solidified the nutrient medium with Gelrite (Ditengou et al., 2015). Here, we exposed seedlings grown on AM with either Gelrite or Phyto Agar and variable pH-values to volatiles of L. bicolor (Figure 12C). The primary root length of $A$. thaliana seedlings decreased with decreasing $\mathrm{pH}$ in both growth media (Figure 12C). Roots of seedlings grown on Gelrite were shorter and developed fewer lateral roots under control conditions compared to roots grown on Phytoagar. Furthermore, some of the seedlings grown on Gelrite developed two primary roots, which was not observed on Phyto Agar.

We observed the strongest root growth at $\mathrm{pH}$ values of 5.8 and 6.3 on Phytoagar of plants grown with and without L. bicolor. Plants grown on Gelrite showed the strongest root growth at $\mathrm{pH}$ of 6.3. Seedlings grown on Phyto Agar showed no difference in growth between pH 5.8 and 6.3. However, plants looked healthier on Phytoagar than on Gelrite. We compared Arabidopsis root and LR growth of plants exposed to L. bicolor volatiles treated to untreated plants and saw differences on Gelrite with pH 6.3 and on Phyto Agar with $\mathrm{pH}$ 6.3. However, the LR response to volatiles was stronger in plants grown on Phytoagar at pH 5.8 than on Gelrite. Therefore, we conducted further experiments on AM supplemented with Phyto agar at pH 5.8. 
A

B Aluminum

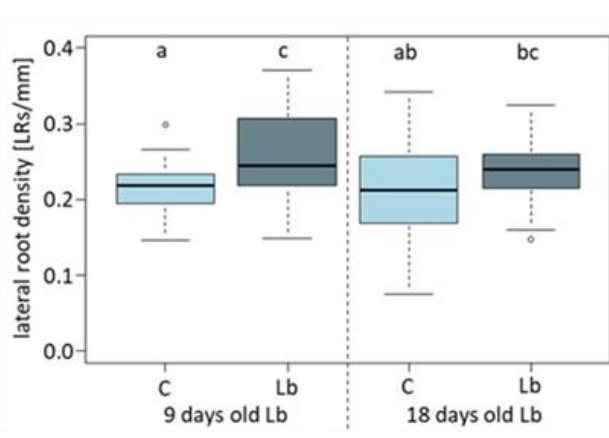

dish

Cellophane

C
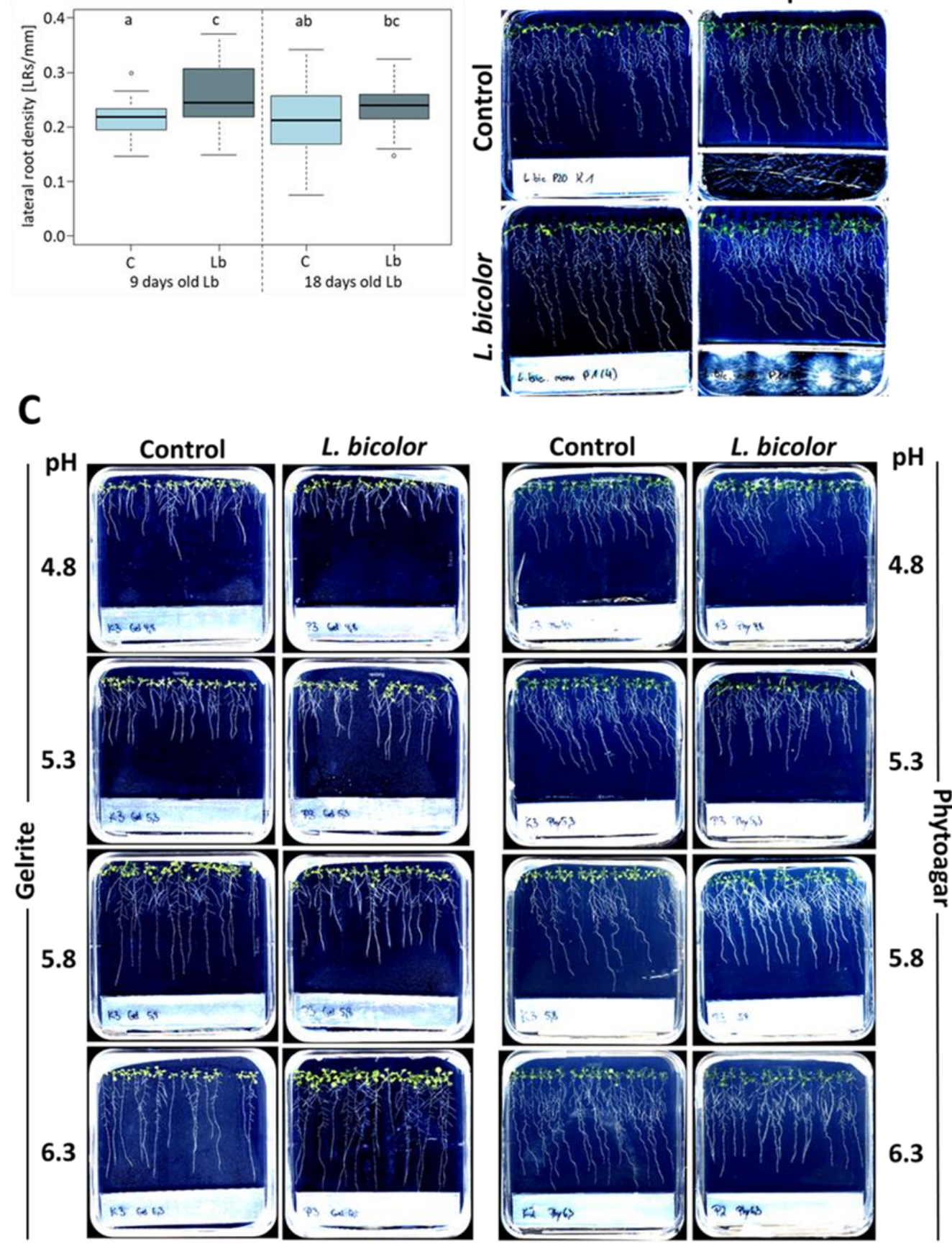

Figure 12. Legend on next page. 
Figure 12. Arabidopsis LR growth under different experimental conditions A) Influence of the age of fungal mycelium on lateral root production. Four-day-old A. thaliana seedlings were exposed to 9-day- or 18-day-old L. bicolor for 7 days or were grown without fungus (control). Per condition, 8 petri dishes with 15 seeds each were prepared, but not all seeds germinated. LR density was determined and statistically analyzed with an ANOVA. Different letters above the bars indicate significant differences at $p<0.001$, according to post hoc Tukey's test. $n=50 \pm 3$ plants per condition and age of the fungus. Since not all seeds germinate, number of seedlings varied. C, control; Lb, L. bicolor; B) Arabidopsis root growth with differentially grown L. bicolor. The fungus was grown on cellophane foil on P20 medium or directly on P20 medium in an aluminum tray for 12 days. LR formation was assessed visually. Typical examples are shown. In total 5 petri dishes with 15 seeds per dish per condition were investigated. C) Arabidopsis growth on Gelrite and Phytoagar. A. thaliana plants were grown on AM supplemented with either Gelrite $\left(12 \mathrm{~g} \mathrm{l}^{-1}\right)$ or Phytoagar $\left(8 \mathrm{gl}^{-1}\right)$. The media were adjusted to different $\mathrm{pH}$-values. Four-day-old seedlings were exposed to volatiles of 12 -days-old L. bicolor. LR formation was assessed visually. Typical examples are shown. In total 3 petri dishes with 15 seeds per condition were investigated.

\subsubsection{L. bicolor was supported with Farnesyl pyrophosphate to stimulate emission of volatiles and enhance LR formation in A. thaliana}

Farnesyl pyrophosphate (FPP) is a precursor of sesquiterpenes (Davis and Croteau, 2000; Sallaud et al., 2009). Since the sesquiterpene, (-)-thujopsen stimulates LR formation (Ditengou et al., 2015) we expected that L. bicolor produces and emits higher amounts of volatiles, when FPP was added. This might result in increased LR formation of A. thaliana compared with untreated fungal cultures. FPP was diluted to different concentrations from $1 \mathrm{~nm}$ to $100 \mu \mathrm{m}$ and added on top of 14-day-old the fungal mycelium. Four-day-old seedlings were exposed to volatiles of treated and untreated fungi for 7 days (Figure 13). For quantitative analysis, LR densities were determined. We did not observe that $A$. thaliana plants produced more lateral roots when the fungus was supplemented with FPP (Figure 13). As expected, plants without L. bicolor formed fewer lateral roots compared to the seedlings exposed to fungal volatiles.

This result suggests either that enhanced LR formation is independent on the concentration of volatiles or that external FPP does not affect sesquiterpene production. 


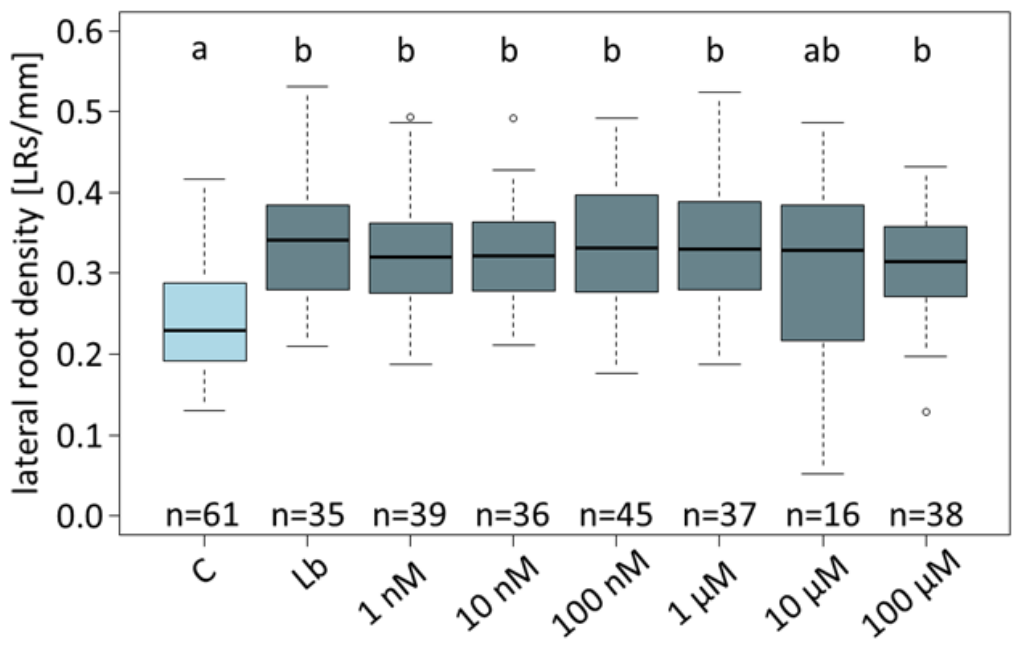

Figure 13. Addition of Farnesyl pyrophosphate to L. bicolor. Twenty-five $\mu$ l Farnesyl pyrophosphate of different concentrations, was added to each fungal plug. Four-day-old seedlings were exposed to 12-day-old L. bicolor without FPP (Lb) or with different concentrations of FPP ( $1 \mathrm{nM}, 10 \mathrm{nM}, 100 \mathrm{nM}, 1 \mu \mathrm{M}, 10 \mu \mathrm{M}, 100 \mu \mathrm{M})$. As control, plants were grown without fungi (C). For each condition, 5 petri dishes with 15 seeds each were prepared. Since not all seeds germinate, number of seedlings varied and indicated by $n=$ in the figure. The experiment was performed once. For statistical analysis, an ANOVA was performed. Different letters above the bars indicate significant differences at $p<0.001$, according to post hoc Tukey's test.

\subsection{Screens of Arabidopsis mutants potentially involved in volatile- induced lateral root formation}

\subsubsection{Arabidopsis thaliana cysteine-rich protein kinases (CRKs) homologs in Populus trichocarpa}

Cysteine-rich protein kinases (CRKs) are a subfamily of receptor like kinases (RLKs) comprising 44 members in Arabidopsis thaliana (Wrzaczek et al., 2010). To find out whether this subfamily structure is conserved in poplar, we identified putative CRKs in Populus trichocharpa by sequence homology with Arabidopsis and generated a phylogenetic tree for comparative analyses of CRKs in our two plant species.

For this purpose, the A. thaliana CRK protein sequences, excluding the putative pseudogene CRK35 and the truncated CRK9 (Bourdais et al., 2015), were collected in the Phytozome 12 database provided by JGI. The Blast tool of the JGI website (https://phytozome.jgi.doe.gov/pz/portal.html\#!search?show=BLAST) was used to identify the poplar proteins. Two-hundred-and-two putative CRK homologs were found 
in P. trichocarpa. Their protein sequences were aligned to the $A$. thaliana protein sequences. The characteristic DUF26 domain of CRKs was annotated and P. trichocarpa proteins without this domain where excluded from further analysis. Sixty-four proteins remained. Those with the ten smallest e-values (from 0 to $4.00 \mathrm{e}-105$ ) were considered for the construction of the phylogenetic tree (Figure 14). The protein identities between the poplar CRKs and the Arabidopsis CRKs ranged from $38 \%$ to $67 \%$. Bourdais et al. (2015) found five distinct subgroups based on the amino acid sequences of the coding region. Groups were defined based on the intracellular kinase domain and the extracellular region (Bourdais et al., 2015). In agreement with this finding, we identified the same five distinct groups for Arabidopsis. Group I constitute a basal clade which forms a sister group distinct from groups II-V (Bourdais et al., 2015). Homologous poplar proteins were found for $A$. thaliana groups I, II and V. The P. trichocarpa protein sequences of CRKs in group I showed a higher sequence similarity to $A$. thaliana CRK proteins than the $A$. thaliana proteins among each other. In contrast, the CRK protein sequences in group II and V were more similar within each plant species.

The phylogenetic tree showed that $P$. trichocarpa CRK proteins of group I, II and V had similar protein sequences as A. thaliana CRKs. For Arabidopsis group III and IV, no homologous proteins were found in P. trichocarpa.

\subsubsection{CRKs involvement in L. bicolor volatiles perception in A. thaliana}

In order to test whether CRKs have a function in the volatile-induced LR formation, we screened the CRK T-DNA insertion library provided by Bourdais et al. (2015). The library encompassed 42 out of 44 CRKs, excluding the putative pseudogene CRK35 and the truncated CRK9 (Bourdais et al., 2015). The mutant lines were exposed to L. bicolor without physical contact. For the experimental set up, L. bicolor grew on P20 medium in aluminum dishes. The dishes were transferred on top of the AM medium on which the Arabidopsis seedlings were growing (chapter 2.1.4; Figure 15A; Figure S1_CRKs). This way we ensured no physical contact between plants and fungus. 


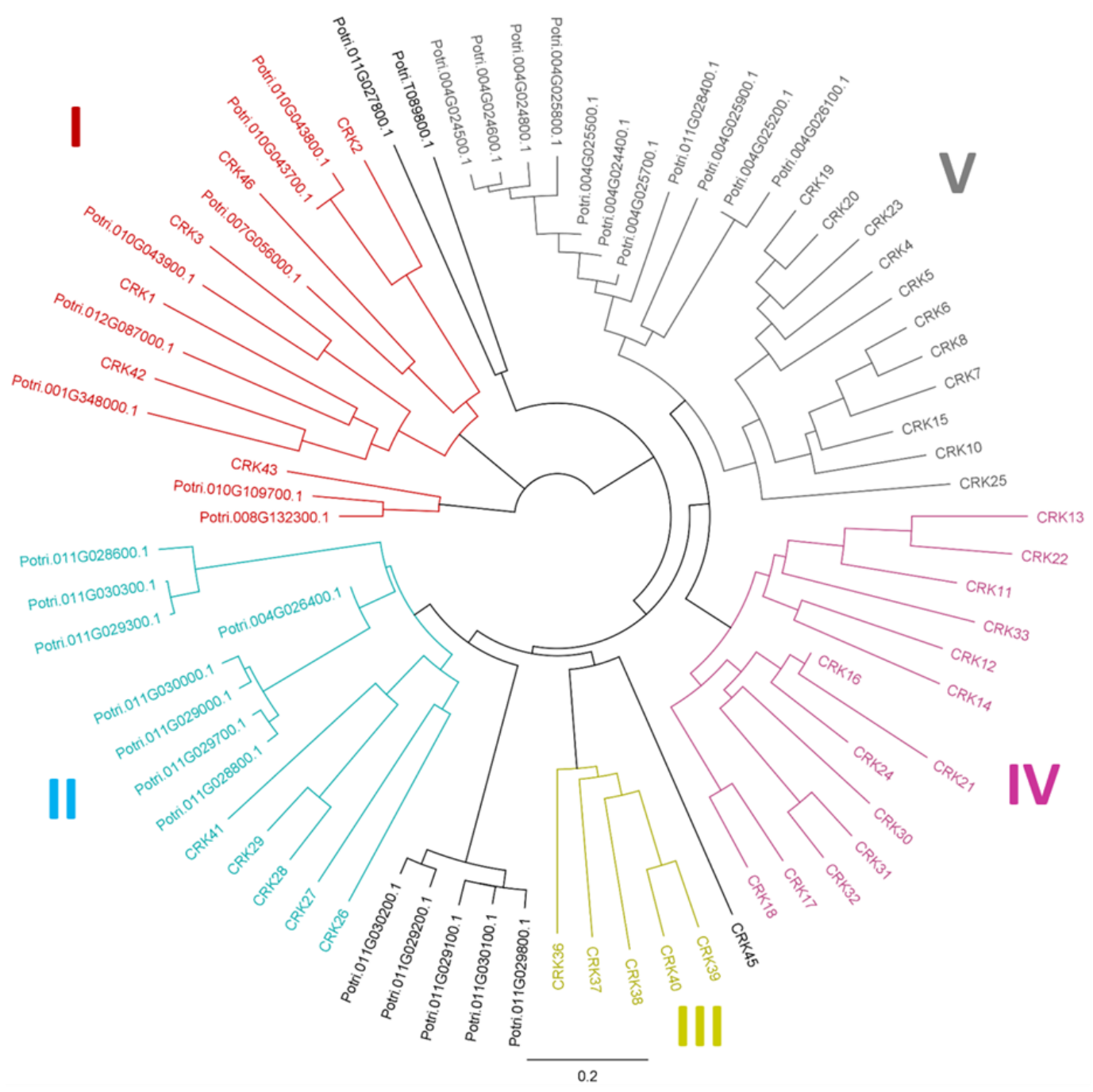

Figure 14. Phylogenetic tree of 42 CRKs in $A$. thaliana and 34 CRKs in P. trichocarpa. Protein sequences of CRKs of both plant species were aligned using Clustal W Alignment. The phylogenetic tree was estimated with Geneious Prime (chapter 2.5). Based on the protein sequence, five distinct groups are formed (Bourdais et al., 2015). Each group is presented in a different color: CRKs shown in red belong to group I, CRKs shown in blue belong to group II, CRKs shown in yellow belong to group III, CRKs shown in violet belong to group IV, CRKs shown in grey belong to group IV.

We determined lateral root (LR) densities of 38 to 143 primary roots per treatment and experiment from several pre-selected lines (Figure 15B-H). The reasons for the selection of these lines were high similarity to poplar CRKs or phenotypes that suggested an involvement in response to volatiles, as detailed below. It is known that Arabidopsis wildtype responds to volatiles of $L$. bicolor with increased LR formation (Ditengou et al., 2015). 
A
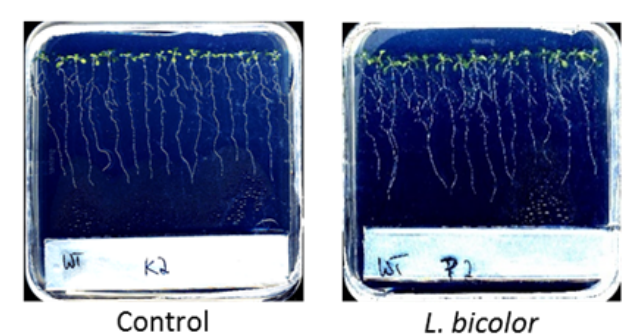

E

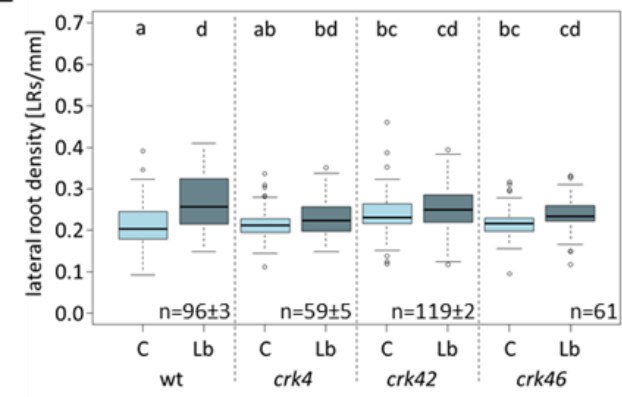

B

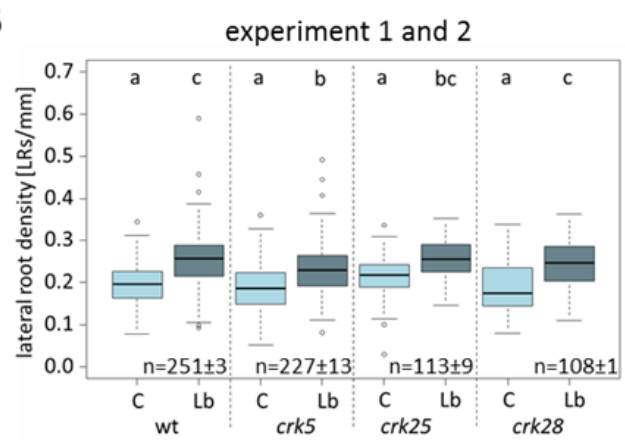

C

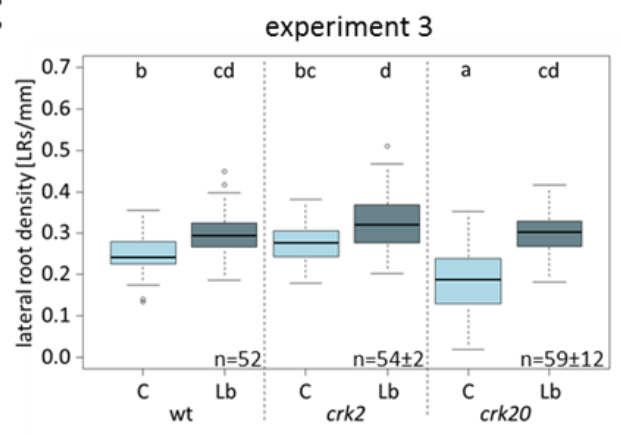

D

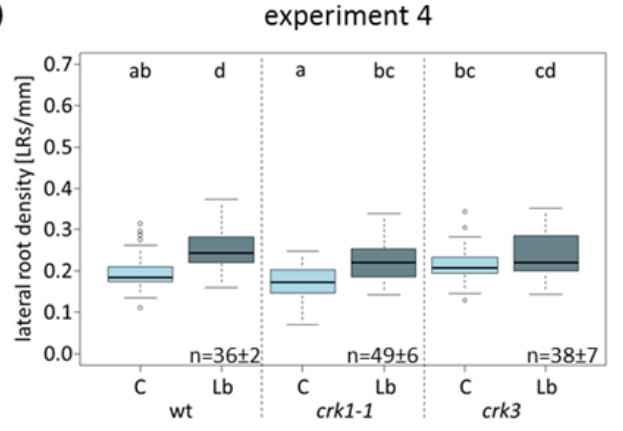

$\mathbf{F}$

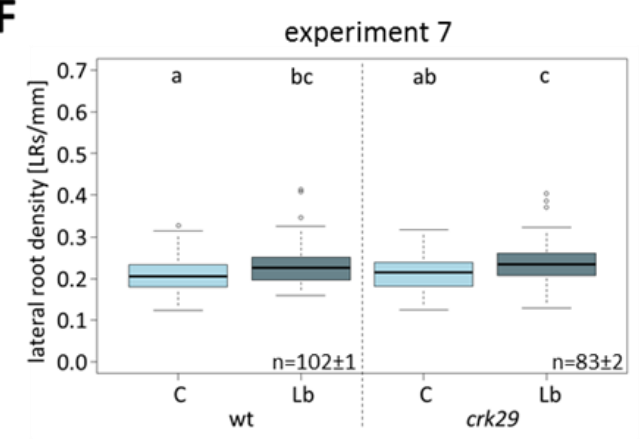

G

H

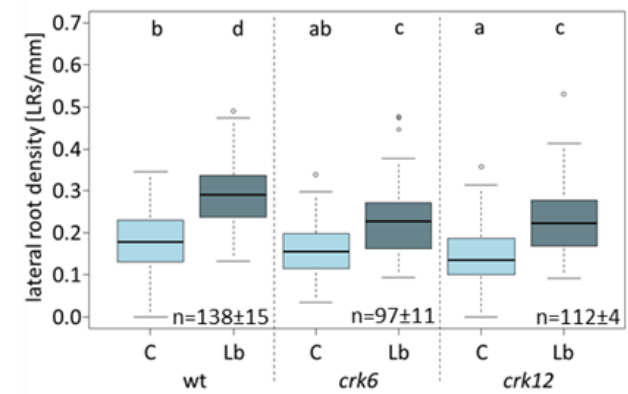

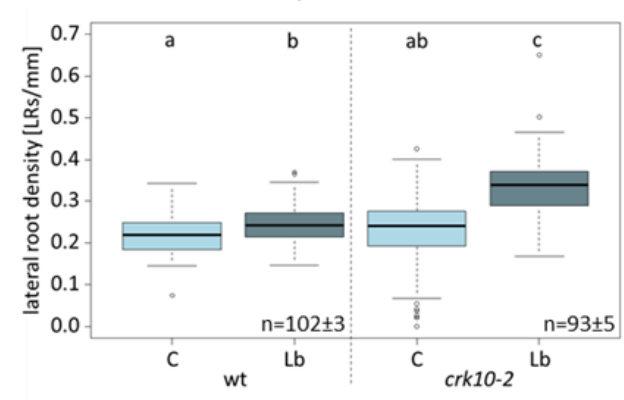

Figure 15. Legend on next page. 
Figure 15. Lateral root density of CRK mutants exposed to L. bicolor volatiles. A) Experimental set up. Fifteen A. thaliana seeds were put on AM. L. bicolor was grown on P20 in aluminum trays for 14 days. The control composed of P20 in aluminum dishes. Six to 10 Petri dishes were prepared per condition, line, and experiment. Since not all seeds germinated and the number of petri dishes varied, the number of plants varied as well. Co-cultivation took place for 11 days. B-H) LR density of crk T-DNA insertion lines (named according to Bourdais et al., 2015) and wildtype (wt) plants under control conditions (C) and exposed to volatiles of L. bicolor ( $\mathrm{Lb}$ ). Mutant lines were tested in independent experiments. LR density was determined from different numbers of plants (n). In B) and E) data from two independent experiments were compiled. For statistical analysis, an ANOVA with number of experiments as random factor was performed. Different letters above the bars indicate significant differences at $p<0.05$, according to post hoc Tukey's test. $n$, number of plants per treatment.

If the response to $L$. bicolor volatiles involved a distinct CRK protein, the LR density of its corresponding mutant line was expected to be unresponsive to L. bicolor volatiles, while LR density of the wildtype increased.

The selected lines originated from different branches of the phylogenetic tree. Crk2, crk5, crk20, crk25 and crk28 were chosen and examined in three independent experiments (Figure 15B and C; Figure S1_CRKs). Pathogenic bacteria affected the immunity of these mutants (Bourdais et al., 2015). Although L. bicolor is not a pathogen, the fungus might trigger similar molecular responses, since symbionts and pathogens use identical or overlapping molecular mechanisms for host-colonization (de Souza et al., 2016).

Furthermore, we examined $A$. thaliana mutants compromised in CRK proteins which were the closed homologs to $P$. trichocarpa proteins. Therefore, we selected crk1-1, ckr3, crk42 and crk46 from group I for testing in three independent experiments (Figure 15D and E; Figure S1_CRKs). Furthermore, we determined the LR density of crk29 (group II) and crk10-2 (group V), because visual assessment indicated an increased LR formation of treated roots compared to treated roots of wildtype plants (Figure 15F; Figure S1_CRKs). In addition, we examined crk6 (group V) and crk12 (group IV) in two replicates (Figure 15H; Figure S1_CRKs). CRK6 protects against apoplastic oxidative stress (Idänheimo et al., 2014), suggesting that it is involved in ROS signaling and might be a promising gene for involvement in volatile signaling (Ditengou et al., 2015). 
In each experiment, wildtype plants (ecotype Col-0) were included as control to accommodate for variability among different sets of experiments (Figure 15B-H). For example, upon L. bicolor treatment the LR density increased 1.3-fold in experiment 4 but only 1.1- fold in experiment 8 (Figure 15D and G). The LR density of non-treated roots also varied. For instance, under control conditions the LR density of wildtype plants was $0.18 \mathrm{LRs} / \mathrm{mm}$ in experiment 9 and $0.25 \mathrm{LRs} / \mathrm{mm}$ in experiment 3 (Figure $15 \mathrm{H}$ and C). Most CRK mutant lines presented in Figure 15B-H showed a response to L. bicolor volatiles that was indistinguishable from that of the wildtype. However, crk3, crk4, crk42, crk46 and crk10-2 behaved differently to wildtype roots. The response to L. bicolor volatiles of $c r k 3, c r k 4, c r k 42$ and $c r k 46$ roots was less intense compared to the wildtype (Figure 15D and E). In contrast to the wildtype, these crk lines showed no significant difference in LR density between L. bicolor exposed and non-treated roots. In addition, the LR density of crk4, crk42 and crk46 non-treated roots was higher compared to wildtype non-treated roots (Figure 15E). Volatiles of L. bicolor induced a trend towards enhanced LR density in crk3, crk4, crk42 and crk46.

In contrast to our hypothesis, crk10-2 showed 1.35-fold increased LR density compared to wildtype plants co-cultivated with L. bicolor (Figure 15G). Under control conditions, we did not observe a difference in LR density between wildtype and crk10-2 roots. Thus, the response to L. bicolor volatiles in ckr10-2 roots is enhanced compared to wildtype roots.

To corroborate these observations, three additional independent T-DNA insertion lines of CRK10 were screened: $c r k 10-1$, crk10-3, and crk10-4. Visual assessment of LR density of those lines did not uncover any obvious difference in LR development compared to the wildtype (Figure 16). Therefore, we did not follow up CRK10 further on.

All other crk mutant lines were exposed to volatiles as described (chapter 2.6.1) and visually assessed in comparison with the wildtype (Table 17 and Figure S1_CRKs). None of those CRK mutant lines showed a clear difference compared to wildtype plants when exposed to $L$. bicolor volatiles. This result indicated that single CRKs are not involved in volatiles perception or volatile-dependent stimulation of LR development. 


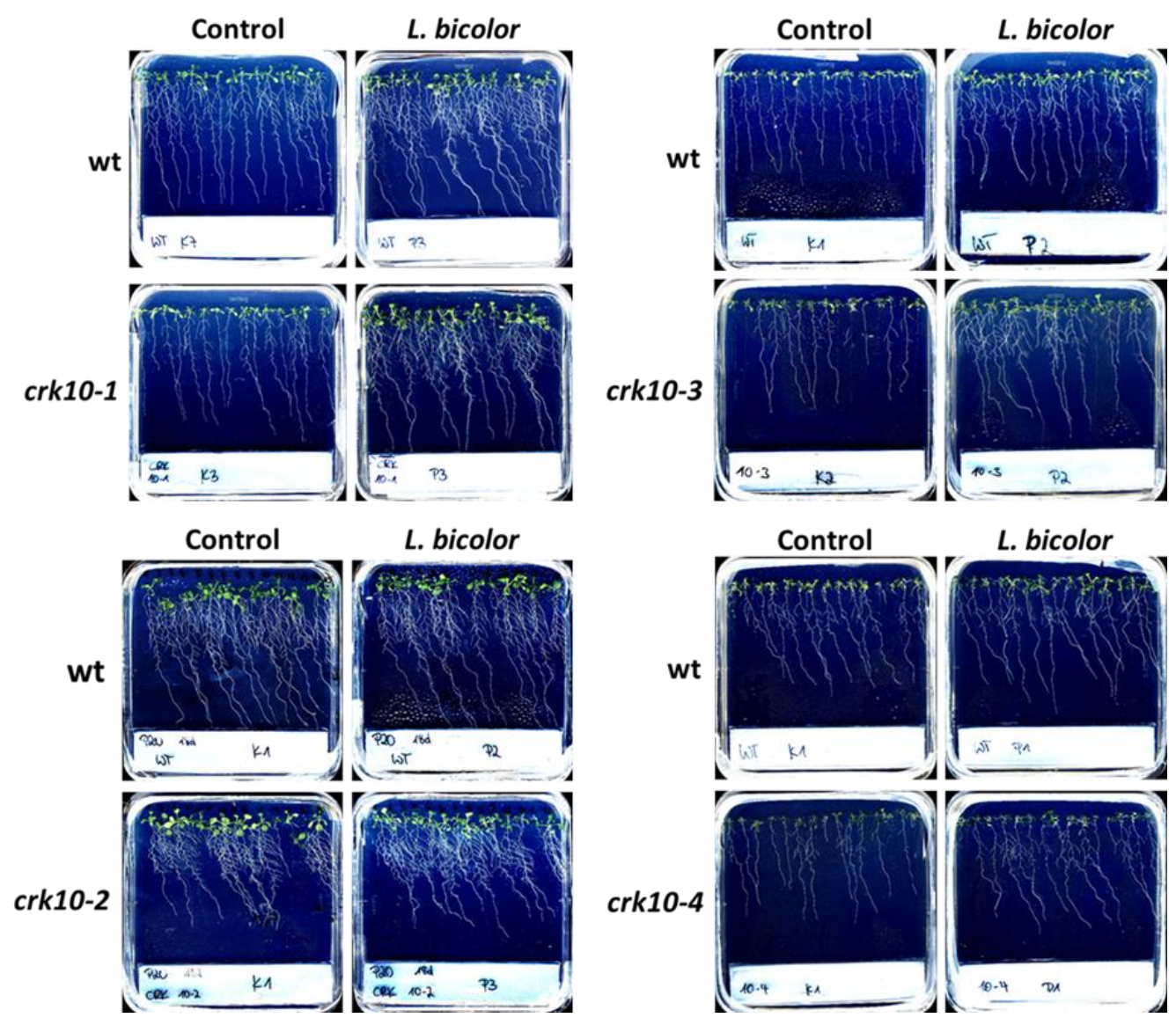

Figure 16. Arabidopsis wildtype and crk10 T-DNA insertion lines exposed to volatiles of L. bicolor for 11 days. Each line was investigated in a different experiment. Therefore, each line was compared to the wildtype plants in the same experiment. All lines showed enhanced LR development when exposed to volatiles. Each panel shows a typical example. Four petri dishes with 15 seeds each per line and condition were examined. The LR density of crk10-2 was quantified, and the data are shown in Figure 15. 
Table 17. Visual assessment of CRK-mutant screens. Mutant lines and wildtype plants (wt) were exposed to L. bicolor volatiles of 14-day-old fungus. After 11 days LR density was determined quantitatively (by counting the LRs and measuring the primary root length) or qualitatively by visual comparison with Arabidopsis wildtype plants and untreated plants in the same experiment. We examined whether the mutant lines showed similar, decreased or increased LR densities of treated roots compared to nontreated roots. The experiment was repeated for promising lines. $0=$ mutant line behaved like wildtype plants, -1 = mutant line showed decreased LR density compared to wildtype, $+1=$ mutant line showed increased LR density compared to wildtype plants, $-1 / 0=$ unclear response

\begin{tabular}{|c|c|c|c|c|c|}
\hline \multirow{2}{*}{$\begin{array}{l}\text { Mutant } \\
\text { line }\end{array}$} & \multicolumn{2}{|c|}{ Difference to wildtype } & \multirow{2}{*}{ Mutant line } & \multicolumn{2}{|c|}{ Difference to wildtype } \\
\hline & experiment 1 & experiment 2 & & experiment 1 & experiment 2 \\
\hline crk1-1 & 0 & & crk21-1 & -1 & 0 \\
\hline crk1-2 & 0 & & crk21-2 & 0 & \\
\hline crk2 & 0 & & crk22 & 0 & \\
\hline crk3 & 0 & & crk23-1 & 0 & \\
\hline crk4 & -1 & & crk23-2 & 0 & \\
\hline crk5 & -1 & 0 & crk24 & 0 & \\
\hline crk6 & $-1 / 0$ & 0 & crk25 & 0 & \\
\hline crk7-1 & 0 & & crk26 & 0 & \\
\hline crk7-2 & $-1 / 0$ & 0 & crk28 & 0 & \\
\hline crk8 & 0 & & crk29 & 0 & \\
\hline crk10-1 & 0 & & crk30 & 0 & \\
\hline crk10-2 & +1 & & crk31 & 0 & \\
\hline crk10-3 & 0 & & crk32 & 0 & \\
\hline crk10-4 & 0 & & crk33 & 0 & \\
\hline crk11 & 0 & & $c r k 36$ & 0 & \\
\hline crk12 & -1 & 0 & crk37 & 0 & \\
\hline crk13 & 0 & & crk38 & 0 & \\
\hline crk14 & 0 & & crk39 & 0 & \\
\hline crk15 & 0 & & crk40 & 0 & \\
\hline crk16 & 0 & & crk41 & 0 & \\
\hline crk17 & 0 & & crk42 & -1 & 0 \\
\hline crk19-1 & 0 & & crk43-1 & 0 & \\
\hline crk19-2 & -1 & 0 & crk43-2 & 0 & \\
\hline crk20 & 0 & & crk45 & 0 & \\
\hline & & & crk46 & -1 & \\
\hline
\end{tabular}




\subsubsection{PER45 and PER59 are not involved in response to volatiles of $L$. bicolor}

To investigate the transcriptomic response to volatiles of L. bicolor, we analyzed microarray-data (Anna Schmidt, 2014, Table S2). A. Schmidt exposed 5-day-old A. thaliana plants to L. bicolor volatiles for 10 days. Root-RNA was extracted and used for Affymetrics microarray analysis.

We found 199 genes in total. None of them was significantly regulated. Four genes tended to be upregulated (PER45, PER59, CPA, At4g30170; $p$ values 0.05 to 0.06), the other 195 genes tended to be downregulated (Table S2). Among the upregulated genes, the class III peroxidases, PER45 and PER59, were identified. Peroxidases are responsible for antioxidant defense by removing peroxides (Lazzarotto et al., 2015). In addition, they respond to environmental stress such as wounding, pathogen attack and oxidative stress (Hiraga et al., 2001). Class III peroxidases are suggested to be involved in plant ROS metabolism (Oliveira et al., 2019). Since ROS play a role in volatiles perception (Ditengou et al., 2015), we speculated that these two peroxidases might be involved in the response to volatiles. T-DNA insertion lines of PER45 and PER59 (source: Nottingham Arabidopsis stock center) were confirmed by PCR as described in chapter 2.4. Homozygous Arabidopsis PER45 (SALK_204976C) and PER59 (SALK_027329C) T-DNA insertion lines and the Col-0 wildtype were exposed to volatiles of L. bicolor and assessed visually (Figure 17). No difference was observed between per45 and per59 and wildtype plants. There was no indication, that PER45 or PER59 were directly involved in response to L. bicolor volatiles. Subsequently, these mutants were not studied any longer.

\subsubsection{Germin-like protein 3 (GLP3) is not involved in response to volatiles of $L$. bicolor}

Germin-like proteins (GLPs) are involved in many plant processes, such as development and defense against biotic and abiotic stress (Dunwell et al., 2008). For example, overexpression of GLP1 and GLP2 located in the plasmodesmata disrupts root growth and development in A. thaliana (Ham et al., 2012), indicating GLP involvement in root development. 


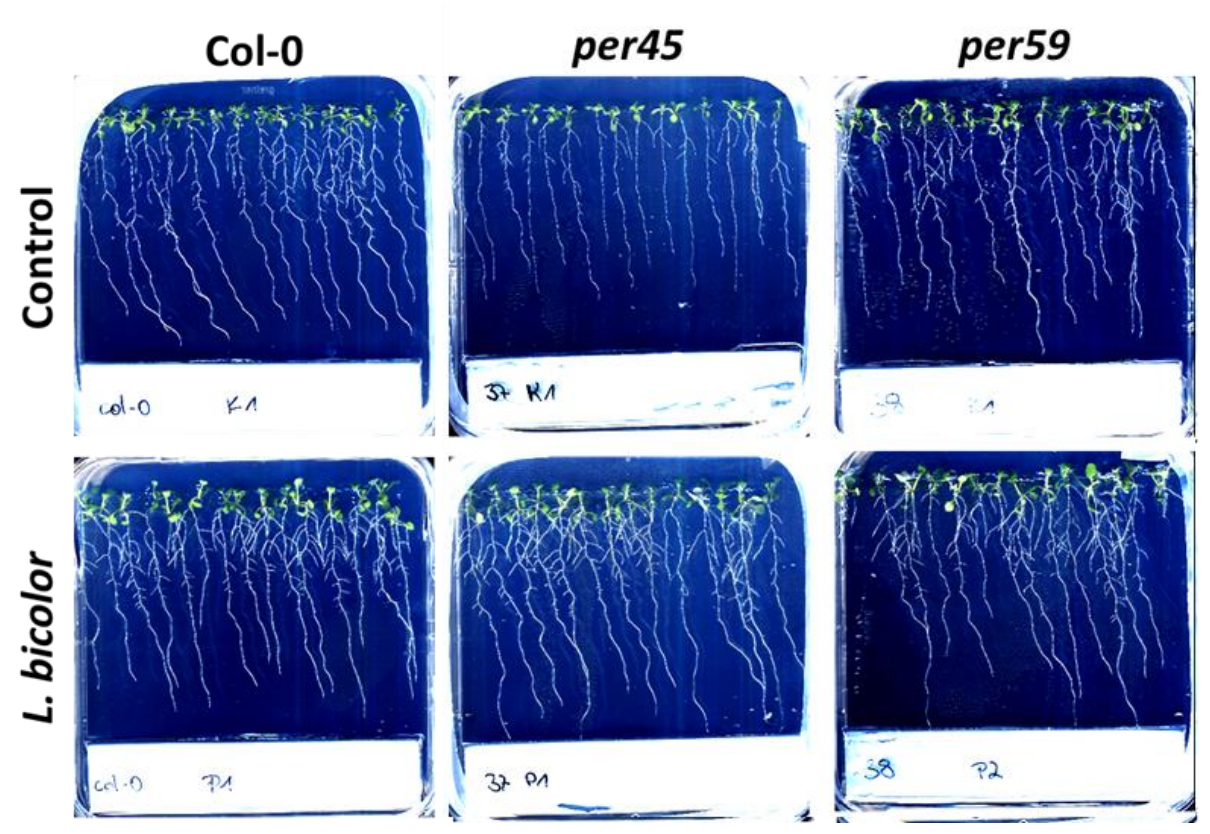

Figure 17. Arabidopsis T-DNA insertion lines per45 and per59 and wildtype Col-0 exposed to volatiles of $L$. bicolor. Genes were selected according to Micro-Array data. L. bicolor was grown on P20 in aluminum trays for 11 days before it was added to the Arabidopsis seeds as described in chapter 2.6.1. Each petri dish presents a typical example. Five petri dishes, each with 15 seeds, per condition and line were observed.

GLP3 is an apoplastic protein that was upregulated in $A$. thaliana plants infected with of Verticillium longisporum (Floerl et al., 2012). The location of GLP3 and the roles of germin-like proteins indicate an involvement in response pathways to volatiles of L. bicolor. Therefore, two T-DNA insertion lines as well as two overexpression lines (created by Drübert, 2011) of GLP3 were exposed to L. bicolor volatiles for 11 days.

Since the T-DNA insertion lines were obtained in the Wassilevskaja ecotype (Ws-2) background, seedlings of this ecotype were also exposed to volatiles. Wildtype plants (ecotype Col-0) were included as controls. Then, 16 to 29 plants per condition and line were scanned and LR densities were determined (Figure 18). Both ecotypes Col-0 and Ws-2 showed the same LR density in non-treated roots $(0.18 \mathrm{LRs} / \mathrm{mm})$. Further, the LR formation was increased 1.7-fold in both ecotypes upon exposure with volatiles. Thus, Col-0 and Ws-2 showed the same response to L. bicolor volatiles (Figure 18B). The two glp3 T-DNA insertion lines and the two 35s::GLP3 overexpression lines showed a similar LR density $(0.179 \pm 0.029 \mathrm{LRs} / \mathrm{mm})$ of non-treated roots compared to Ws-2 non-treated roots. The response to volatiles of the overexpression lines 35s::GLP3 line 5 and 6 and T-DNA insertion glp3 line 20 was indistinguishable from that of wildtype 
plants (Figure 18B). In contrast, glp3 line 18 shows a significantly lower LR density in roots exposed to volatiles. However, the increase of $1.61 \mathrm{LRs} / \mathrm{mm}$ in LR formation upon treatment with volatiles is significant $(p<0.001)$. Furthermore, the GLP3 relative transcript level of line 18 is similar to the wildtype, whereas the transcript level of line 20 is significantly lower (Druebert, 2011). For these reasons, glp3 line 18 were not pursued further, nor were the glp3 line 20 and the overexpression lines.

By analyzing the LR densities, we noticed an aslant primary root growth of all Arabidopsis seedlings. But it was more prominent in plants with the Ws-2 background Figure 18. Since the special growth did not affect our experiment, we did not investigate it further.

\subsubsection{Strigolactone are not involved in response to volatiles of $L$. bicolor}

Strigolactones are a group of phytohormones, which are secreted by plants to indicate the presence of a host to symbiotic fungi or root parasitic plants (Yoneyama et al., 2013; Akiyama et al., 2005). In addition, strigolactones play a role in hormonal crosstalk during root development. They repress LR formation under optimal growth conditions (Kapulnik et al., 2011a; Ruyter-Spira et al., 2011). Consequently, increased LR formation in mutants with defects in strigolactone biosynthesis or signaling may be expected. Ruyter-Spira et al. (2011) examined different mutants involved in strigolactone biosynthesis and signaling. Among them, max4 (more axillary branching 4) and max2 (more axillary branches 2) were investigated. MAX4 is a key factor in strigolactone biosynthesis (Alder et al., 2012; Sorefan et al., 2003), whereas MAX2 is a F-Box-protein, which is the substrate recognition subunit of the Skp-Cullin-F-Box ubiquitin E3 ligase for proteasome-mediated proteolysis (Ishikawa et al., 2005; Johnson et al., 2006; Stirnberg et al., 2002; L. Wang et al., 2015). 

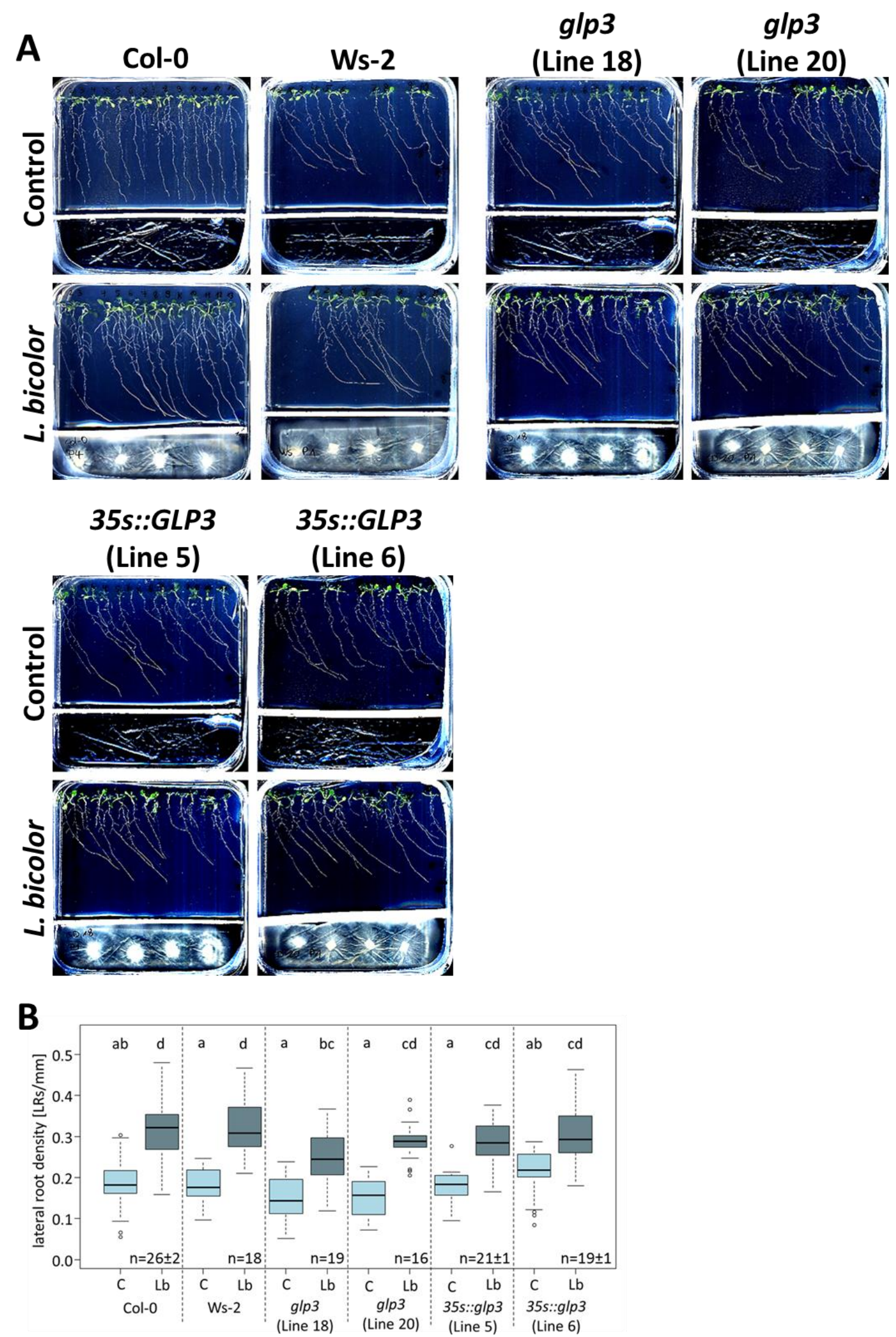

Figure 18. Legend on next page. 
Figure 18. LR density of Arabidopsis glp3 and 35s::GLP3 mutant lines exposed to L. bicolor volatiles. A) Examples of plants exposed to volatiles. All glp3 and $35 s:: G L P 3$ lines were transformed in Ws-2 background. For comparison and control, Col0 was examined. Seeds were exposed to volatiles of 14-day-old L. bicolor for 11 days, according to the protocol in chapter 2.6.2. Each petri dish presents a typical example. Five petri dishes with 15 seeds per dish were prepared per line and treatment. B) LR density of mutant lines exposed to $L$. bicolor volatiles. Since not all seeds germinated the number of analyzed plants per line varied between treatments and lines. Data originated from one experiment. For statistical analysis, an ANOVA was performed. Different letters above the bars indicate significant differences at $p<0.001$, according to post hoc Tukey's test. $n$, number of plants per treatment, C, control; Lb, L. bicolor

Strigolactones bind to MAX2-Skp-Cullin-F-Box ubiquitin E3 ligase complex, which leads to its degradation and expression of strigolactone responsive genes (Soundappan et al., 2015; L. Wang et al., 2015). Apparently, MAX2 plays a crucial role in signaling (Kapulnik et al., 2011a). Ruyter-Spira et al. (2011) showed that the basal LR density in both mutants is increased compared to wildtype plants. Nevertheless, max 2 showed a much higher (45\%) difference in LR density compared to wildtype plants, than max4 (9\%). Max2 and max4 were treated with different concentration of GR24, a synthetic strigolactone analogue. Whereas max4 showed a significant reduction in LR density upon GR24 treatment, LR density of max2 was not affected (Ruyter-Spira et al., 2011). Based on the roles of MAX2 and MAX4 in LR formation, we hypothesized that L. bicolor volatiles affect the strigolactone biosynthesis or signaling in A. thaliana negatively to reduce the repression of $L R$ formation. If increased LR formation is strigolactone biosynthesis or signaling dependent, no effect on LR density of max2-1 and max4-1 seedlings upon treatment with volatiles was expected. Under control conditions lower LR densities in both mutant lines were expected compared to wildtype seedlings. Max-mutants and wildtype seedlings were exposed to volatiles of L. bicolor for 11 days. Wildtype seedlings showed an increased LR density upon treatment with volatiles of L. bicolor (Figure 19). In agreement with enhanced R density found by Ruyter-Spira et al. 2011 in max2 mutant, we also found significantly enhanced LR formation (0.20 LRs $/ \mathrm{mm}$ ) compared to wildtype seedlings under control conditions (Figure 19). In contrast to the expectations, LR density of max2-1 roots exposed to L. bicolor volatiles showed significanlty enhanced LR density (1.26-fold) compared to non-treated roots. 
In contrast to Ruyter-Spira et al. 2011, the max4-1 mutants showed decreased LR formation compared to wildtype plants under control conditions (Figure 19). The max4-1 mutant seedlings responded to volatiles with enhanced LR development. The difference in LR density of control max4 seedlings compared to treated pants is the similar to that found for wildtype plants ( 1.45 times). Thus, an involvement of MAX2 and MAX4 and strigolactone biosynthesis in response to volatiles of L. bicolor is unlikely.

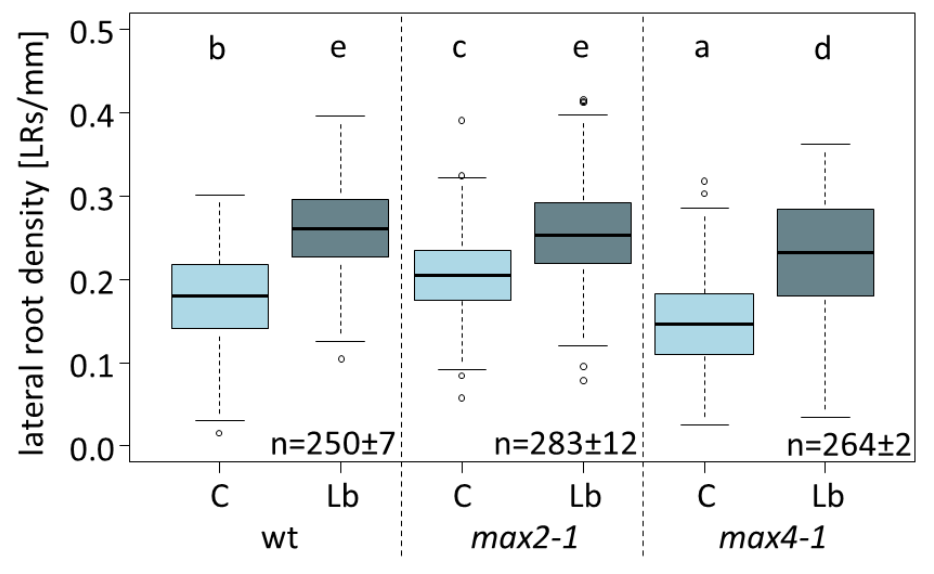

Figure 19. LR density of $A$. thaliana $\operatorname{max2-1}$ and $\operatorname{max4-1}$ lines. Seeds were exposed to volatiles of 14-day-old L. bicolor for 11 days, according to the protocol in chapter 2.6.2. Ten petri dishes with 15 seeds per dish were prepared per line and treatment. Since not all seeds germinated the number of analyzed plants per line varied between treatments and lines as indicated by $\mathrm{n}$ in the figure. Data originated from 3 independent experiments. For statistical analysis, an ANOVA with number of experiments as random effect was performed. Different letters above the bars indicate significant differences at p<0.001, according to post-hoc Tukey's test. C, control; Lb, L. bicolor 


\subsection{Testing the suitability of the pGATA23::n/s-GFP-GUS reporter as a marker for volatile-induced LR formation}

The GATA23 transcription factor specifies pericycle cells to become lateral root founder cells prior to lateral root formation (De Rybel et al., 2010). Furthermore, GATA23 is regulated by auxin, which plays a crucial role in lateral root formation (Casimiro et al., 2001; Laskowski et al., 1995; De Rybel et al., 2010). Therefore, De Rybel et al. (2010) used the GATA23 promoter to construct GFP-GUS reporter line. They found that the promotor activity was confined to early stages of LR development (stage I-III primordia). Thus, the GFP signal decreased with progressive LR development. De Rybel et al. (2010) also supplemented the reporter construct with a nuclear localization signal (nls), resulting in PGATA23 regulated GFP expression in nuclei of stage I-III primordia cells (De Rybel et al., 2010).

Here, we investigated whether the pGATA23::nls-GFP-GUS reporter could serve as an early indicator for volatiles-dependent LR formation. We used the appearance of GFP fluorescence to analyze primordia density at early stages of LR development and in response to exposure to $L$. bicolor volatiles. For this purpose, 4-day-old $A$. thaliana pGATA23::nls-GFP-GUS and wildtype (Col-0) seedlings were exposed to volatiles of 14-day-old L. bicolor for 7 days (chapter 2.7).

Primordia were counted under a fluorescence microscope $0,1,2,4$ and 7 days after exposure to fungal volatiles and in controls. Under this device only small segments of detached roots could be observed. We defined primordia as the accumulation of GFP expressing nuclei in the pGATA23::n/s-GFP-GUS reporter lines. In 4-day-old $A$. thaliana seedlings not yet exposed to fungal volatiles, primordium development and expression of GFP had just started (Figure 20A). The fluorescence signal was partially low. The fluorescence signal was mainly present in the middle of the roots, which was expected when the GFP expressing nuclei were in pericycle cells, the origin of LRs (Casimiro et al., 2003; Dolan et al., 1993; Dubrovsky and Rost, 2012).

On day 1 of exposure to volatiles, the fluorescence signal was increased compared to day zero (Figure 20B). During the time course of 7 days exposure to fungal volatiles an accumulation of nuclei, as an indicator for primordia initiation, was often observed but difficult to distinguish from other GFP expressing nuclei (as shown in Figure 20B for e.g. day 4). 
In order to get an overview on the distribution of GFP expressing nuclei all over the root, the whole root was photographed segment by segment and merged to one image (Figure 20C). However, this approach resulted in brighter fluorescence in the center and lower intensities at the edges and thus a regular wavy pattern of the merged figure. The GFP fluorescence was not strong enough be visible in overview photos. Therefore, we were not able to analyze primordia distribution across the whole root.

We studied the time course of LR and primordia densities of the pGATA23::n/s-GFP-GUS line in response to exposure to L. bicolor volatiles in freshly harvested roots (Figure 20D-F). We observed the whole root each day, but the GPP signal and thus the primordia were typically found between the youngest LR and the basal meristem (Figure 1). The LR density was slightly but not significantly enhanced in roots treated with volatiles for 4 and 7 days compared to roots grown without volatiles for the same time (Figure 20D). This might be due to the low number of plants used in this experiment (4 to 5). In general, LR density increased over time, while primordium density decreased due to growth of primary roots but low increase primordium number (Figure 20D).

During the time course experiment with the pGATA23::n/s-GFP-GUS line, we exposed additional 4-day-old plants of the pGATA23::n/s-GFP-GUS line and the wildtype to L. bicolor volatiles for 7 days to compare the response of the pGATA23::nls-GFP-GUS line with that of the wildtype plants. The pGATA23::n/s-GFP-GUS line responded to fungal volatiles with enhanced LR formation similar to wildtype plants (Figure 20G). However, under control conditions the LR density of the pGATA23::n/s-GFP-GUS line was 1.17-fold higher than that of wildtype seedlings. 


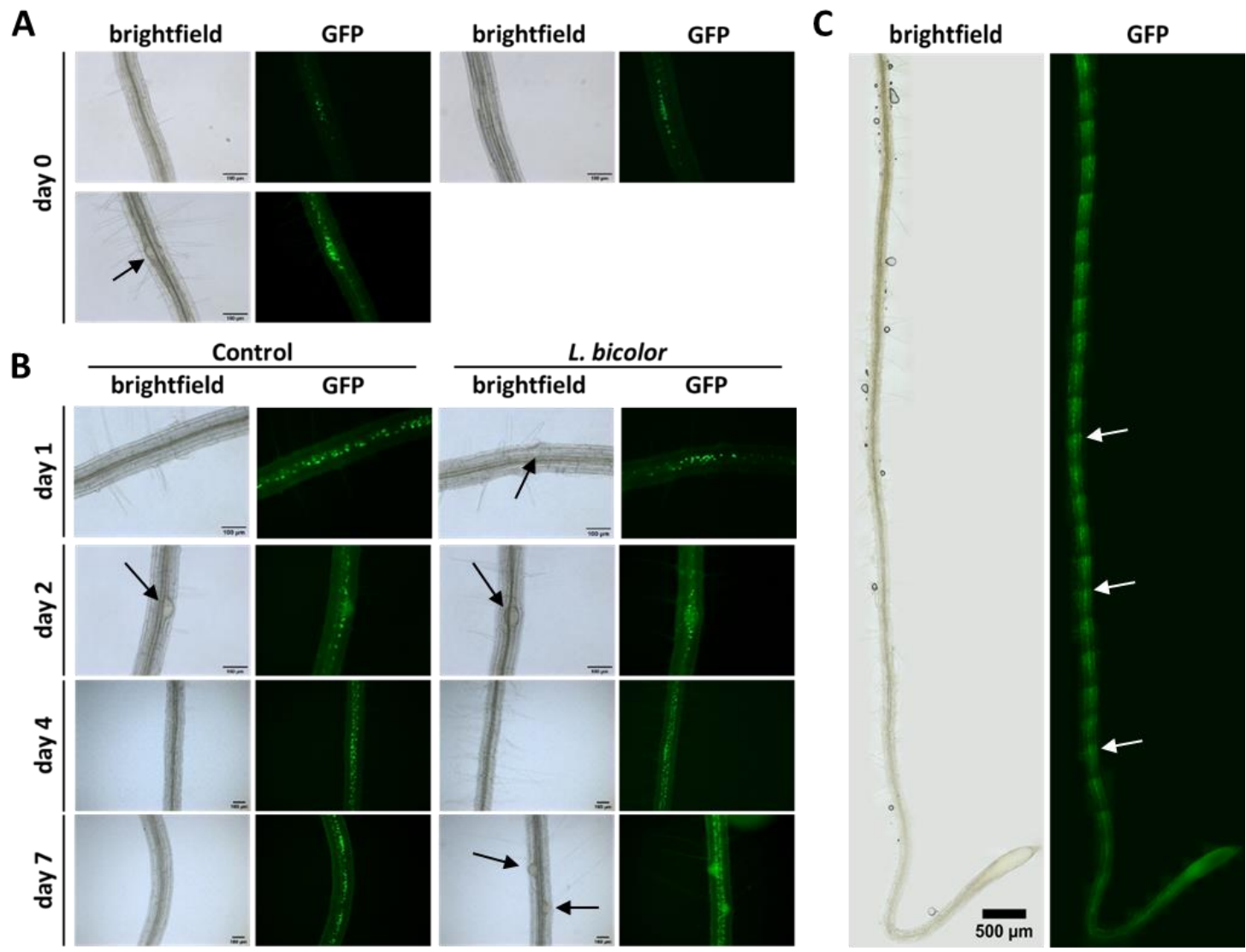

D

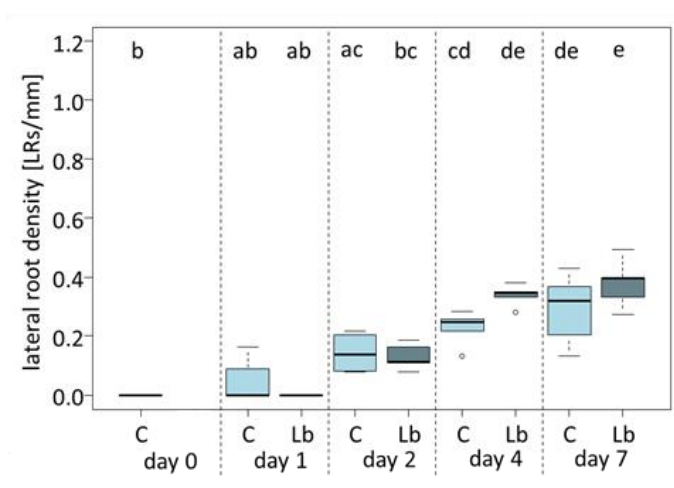

E

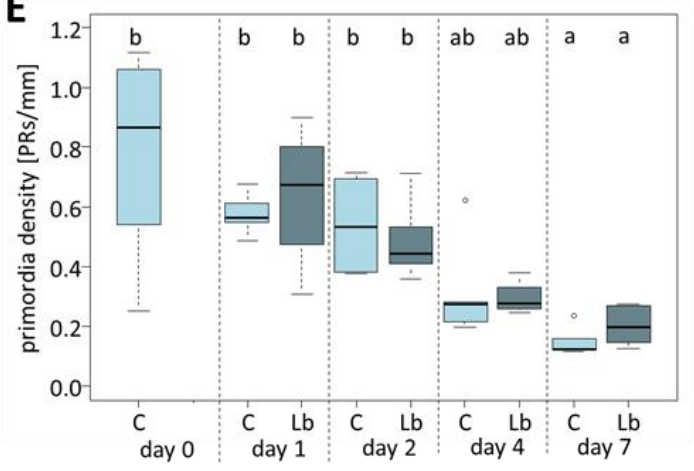

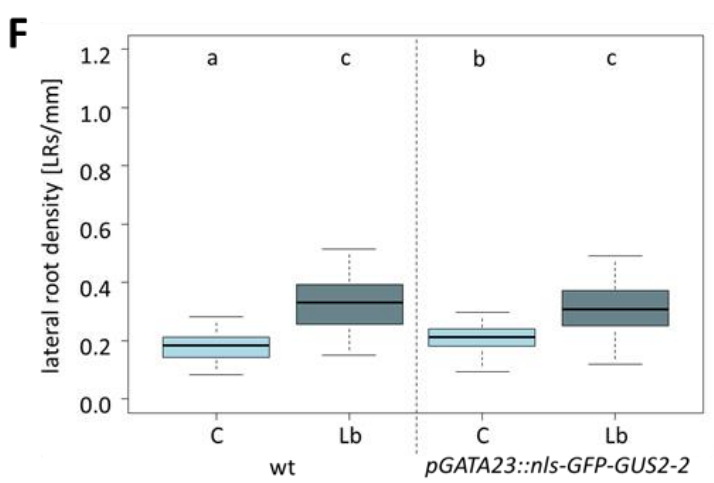

Figure 20. Legend on next page. 
Figure 20. Fluorescence microscopy of primordia in the $A$. thaliana reporter line pGATA23::n/s-GFP-GUS. A) Different stages of LR formation of 4-day-old seedlings at day 0 of exposure to volatiles. B) Typical examples of GFP expressing nuclei at different time points of exposure to volatiles of $L$. bicolor. Scale bar $=100 \mu \mathrm{m}$. Arrows show visible primordia. C) Example of a larger part of a root after 4 days of exposure. Twenty-six individual figures were merged. The regular appearance of bright and dark parts in the figure is an artefact caused by merging (indicated by arrows). Scale Bar $=500 \mu \mathrm{m}$. D) Time course of lateral root (LR) density and F) primordia (PR) density of pGATA23::n/s-GFP-GUS seedlings. We counted LRs and PRs from 5 plants per treatment and time point. Six seeds were grown in one petri dish. The experiment was conducted once. Data were analyzed by an ANOVA. Different letters above the bars indicate significant differences at $p<0.05$, according to post-hoc Tukey's test. G) LR density of wildtype plants compared to pGATA23::n/s-GFP-GUS plants after 7 days of exposure to volatiles. Six petri dishes per line and treatment with 15 seeds per dish were prepared. Since not all seeds germinated the number of analyzed plants per line varied between treatments and lines. For wildtype $73 \pm 2$ plants and for pGATA23::nls-GFP-GUS $65 \pm 4$ plants per treatment were analyzed. The experiment was conducted once. Data were analyzed by an ANOVA. Different letters above the bars indicate significant differences at $p<0.05$, according to post-hoc Tukey's test. C, control; Lb, L. bicolor

Because of the unsolved question regarding the distribution of the primordia in the roots, the experiment was repeated using a fluorescent stereomicroscope for fluorescence detection. This device allowed us to analyze roots without removing them from petri dishes. Thus, it was possible to study the same roots over the whole time-course (chapter 2.7). Besides visualizing the distribution of GFP expressing nuclei, the aim of this experiment was to investigate primordia development with and without exposure to volatiles. It was striking that the GFP expression was higher in curved parts of the root (Figure 21A). Furthermore, GFP expressing nuclei were distributed all over the root, contrary to De Rybel et al. (2010), who showed only primordium nuclei specific GFP signals in their publication. We found GFP expressing nuclei with higher accumulation, indicating primordia predominantly in the meristem and elongation zone of the roots. After 4 days of exposure to volatiles, we noted that most of the GFP expressing nuclei accumulated between the youngest lateral root and the root tip. Nevertheless, it was not possible to distinguish between GFP expressing nuclei from primordia and other GFP expressing nuclei in an appropriate manner, especially at early stages of primordium formation. Consequently, the pGATA23::nls-GFP-GUS reporter 
line was not suitable to investigate primordia at early stages of LR development. Classical counting of LR showed that fungal volatiles stimulated LR densities but in this experiment $L R$ density of the wildtype in the absence fungal volatiles was higher than that of the pGATA23::n/s-GFP-GUS reporter line (Figure 21B) Summarizing the data from the two independent experiments (Figure 20G and 21B) showed that both wildtype and the pGATA23::n/s-GFP-GUS reporter line had similar LR densities in the absence and enhanced LR densities in the presence of fungal volatiles (Figure 21C). This result shows that the pGATA23::n/s-GFP-GUS reporter roots behaved like wildtype roots when exposed to volatiles of $L$. bicolor.
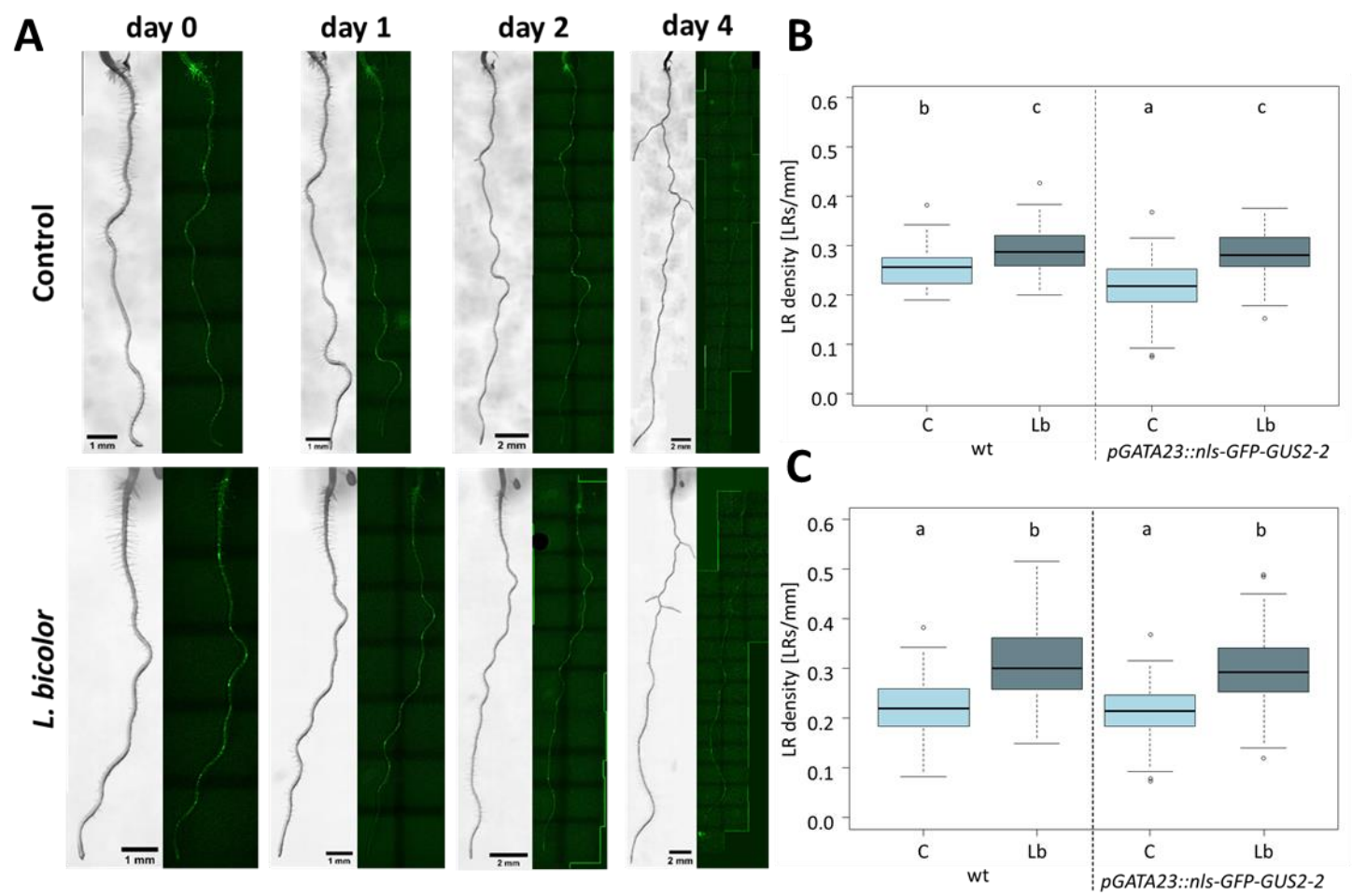

Figure 21. Fluorescence stereo microscopy of whole roots of the $A$. thaliana primordia reporter line pGATA23::n/s-GFP-GUS. A) Representative examples of a root observed at different days during volatile exposure and another root growing in the absence of fungal volatiles. Two petri dishes per condition with 10 seeds per dish were prepared. Eight plants per condition were microscopically analyzed. Scale bar day 0 and $1=1 \mathrm{~mm}$, Scale bar day 2 and $4=2 \mathrm{~mm}$. B) LR density of wildtype plants compared to pGATA23::nls-GFP-GUS plants after 7 days of exposure to volatiles. Twelve petri dishes per line and treatment with 10 seeds per dish were prepared. Since not all seeds germinated the number of analyzed plants per line varied between treatments and lines. For wildtype $74 \pm 2$ plants and for pGATA23::nls-GFP-GUS $82 \pm 1$ plants per treatment were analyzed. The experiment was conducted once. Data were analyzed by an ANOVA. 
Different letters above the bars indicate significant differences at $p<0.001$, according to post-hoc Tukey's test. C) Data of LR densities of two independent experiments were combined (Figure 1G and 2B). For wildtype 148 plants and for pGATA23::nls-GFP-GUS $131 \pm 5$ plants per treatment were analyzed. An ANOVA with replicates as random factor was performed. Different letters above the bars indicate significant differences at p $<0.001$, according to post-hoc Tukey's test. C, control; Lb, L. bicolor

\subsection{Determination of the response dose and time of $A$. thaliana roots to volatiles of $L$. bicolor}

\subsubsection{Response of $A$. thaliana roots is $L$. bicolor mycelium amount dependent}

To investigate whether the enhanced LR formation upon volatile exposure was L. bicolor dose dependent, 4-day-old $A$. thaliana seedlings were exposed to 1, 2, 3 or 4 fungal plugs for 7 days. After 7 days of co-cultivation, we found that one L. bicolor plug was sufficient to increase LR formation in A. thaliana roots significantly by a factor of 1.38 (Figure 22A). The volatiles of two fungal plugs led to higher LR density (1.46-fold) compared to plants, which were exposed to one fungal plug. Volatiles of three and four fungal plugs led to stimulation of LR density similar to that of two fungal plugs (Figure 22A). We concluded that enhanced LR formation was dependent on the amount of L. bicolor mycelium but that already the mycelium of two L. bicolor plugs was sufficient to induce maximum stimulation of LR formation in A. thaliana.

\subsubsection{Two days of exposure to volatiles of $L$. bicolor are sufficient to induce enhanced LR formation in A. thaliana}

Besides the L. bicolor dose, we tested the time period necessary to induce the LR response in $A$. thaliana roots. To address this question, we exposed 4-day-old seedlings to volatiles of four 14-day-old L. bicolor plugs grown in aluminum trays. Plants were exposed for 1, 2, 4 or 7 days to the tray with L. bicolor, which was then removed. The plants were further cultivated in the absence of the fungus until all plants had reached an age of 11 days ( 4 days preculture and 7 experimental days). We found that 2 days of exposure to volatiles of $L$. bicolor were sufficient to induce enhanced LR density in A. thaliana roots by a factor of 1.08 (Figure 22B). However, the longer A. thaliana seedlings were exposed to volatiles, the higher was the difference in LR density between 
control and treated plant. The increase was 1.16-fold after 4 days of exposure and 1.20-fold after 7 days of exposure. Thus, the highest LR density was achieved when the plants had been exposed continuously for 7 days to fungal volatiles (Figure 22B).

A

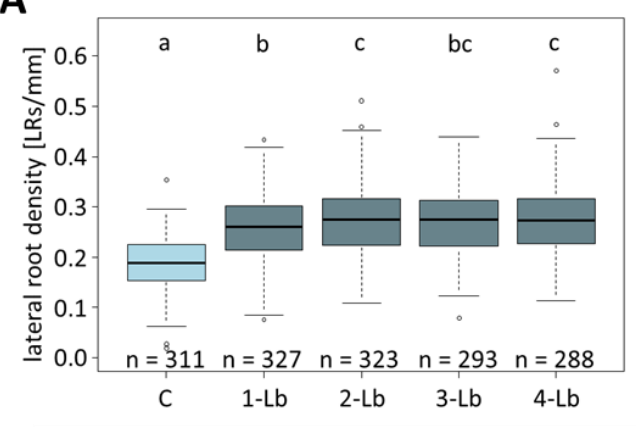

B

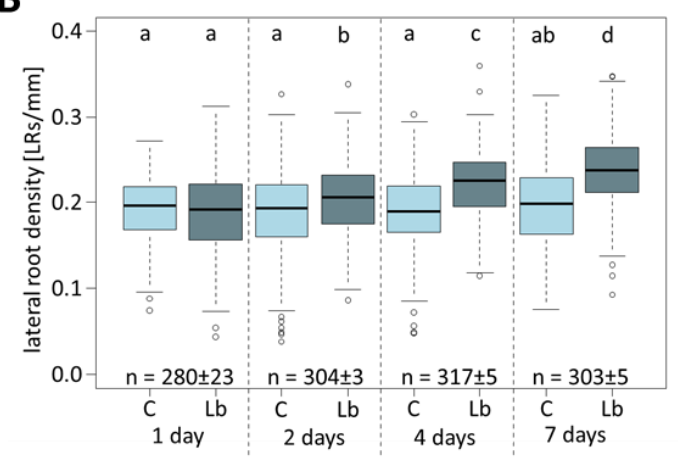

Figure 22. A) LR density of $A$. thaliana roots exposed to volatiles of $1,2,3$ or 4 L. bicolor plugs for 7 days. B) LR density of $A$. thaliana roots exposed to 4 L. bicolor plugs for different time periods. In both experiments, 10 petri dishes per treatment and replicate with 15 seeds per dish were used. Fungal plugs on trays had an age of 14 days. Since not all seeds germinated the number of analyzed plants per treatment varied as indicated by $n$ in the figure. $n$ indicates the number of plants of 3 independent replicates. For statistical analysis, an ANOVA with number of replicates as random factor was performed. Different letters above the bars indicate significant differences at $p<0.001$, according to post-hoc Tukey's test. C, control; Lb, L. bicolor 


\subsection{Influence of $L$. bicolor volatiles on the transcriptome and metabolome of $A$. thaliana and $P . x$ canescens}

\subsubsection{Transcriptional changes $A$. thaliana roots during exposure to $L$. bicolor volatiles for one week}

We exposed 4-day-old A. thaliana plants to L. bicolor volatiles for 0, 2, 4 and 7 days and used roots for transcriptome analyses (chapter 2.10.3). We performed the experiment in three independent replicates. For each replicate, we controlled LR stimulation by fungal volatiles (Figure 23). For this purpose, each experiment contained additional A. thaliana plants that were exposed to volatiles for 7 days and compared to non-treated plants concerning their LR density. In each replicate, the LR density of plants exposed to volatiles was increased compared to plants grown without volatiles (Figure 23). The RNA of the Arabidopsis roots was extracted and sent for sequencing as described in chapters 2.10 .2 and 2.10.3.
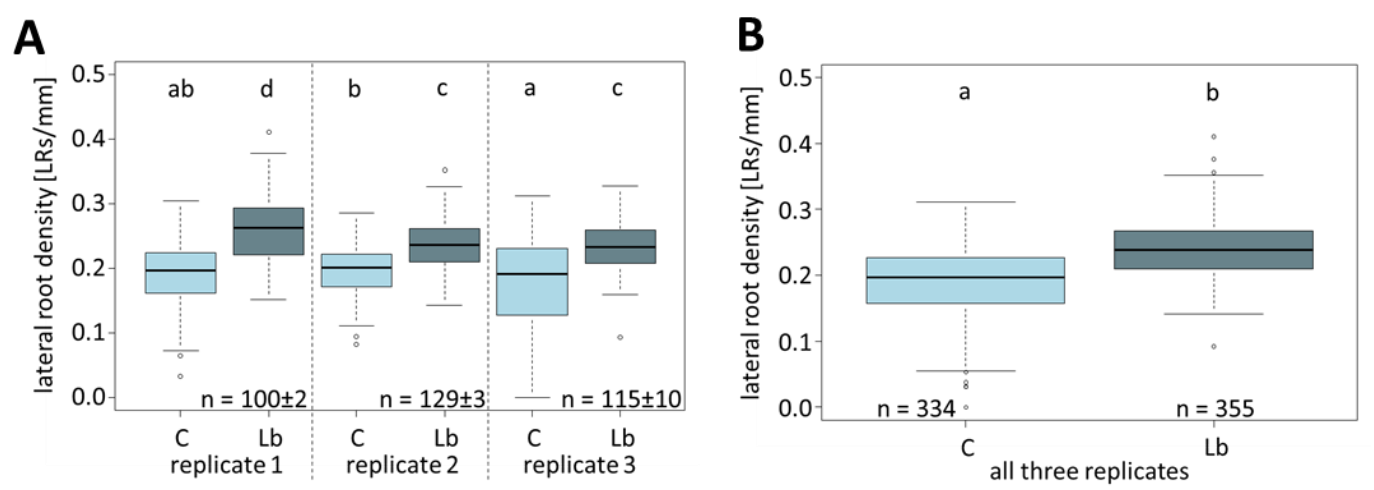

Figure 23. LR density of Arabidopsis wildtype plants grown together with samples for transcriptome analysis in the time-course experiment. A) LR density of each individual replicate. B) Data of all replicates were compiled. 4-day-old seedlings were exposed to volatiles of 14-day-old L. bicolor for 7 days, according to the protocol in chapter 2.1.4. Ten petri dishes per treatment with 15 seeds per dish were prepared. Since not all seeds germinated the number of analyzed plants $(n)$ varied between treatments and replicates. Data originated from 3 independent replicates. For statistical analysis, an ANOVA with number of replicates treated as random factor was performed (in B). Different letters above the bars indicate significant differences at $p<0.001$, according to post-hoc Tukey's test. C, control; Lb, L. bicolor 
Principal component analysis (PCA) of normalized count data showed that the independent replicates clustered together, with exceptions (Figure 24). The transcriptome changed with increasing seedling age (PC1, Figure 24). The effect of exposure to fungal volatiles was less evident and mainly apparent on day 2 and day 4 along PC2 (Figure 24).

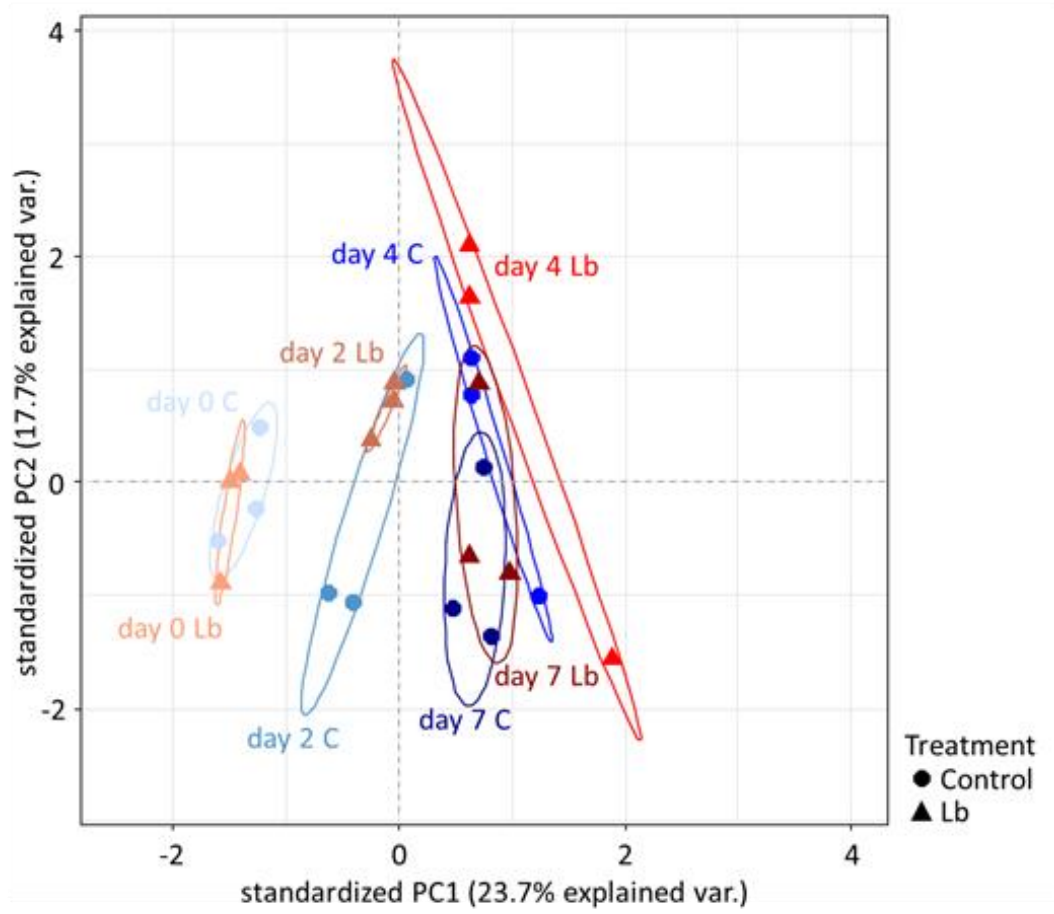

Figure 24. PCA of transcriptomic normalized count data. Transcriptome of whole roots of $A$. thaliana harvested at day $0,2,4$ and 7 of L. bicolor (14-day-old) volatile exposure were considered. PC1 explains separation by time of exposure and PC2 separation by treatment. This dataset included 16,335 transcripts. C, control; Lb, L. bicolor

Samples at day 0 of exposure to L. bicolor volatiles were not separated according to treatment. After 2 days of volatile exposure, genes tended to separate according to their treatment. However, one replicate of the 2-day control samples grouped together with the volatile treated samples of the same day. Replicates of day 4 and 7 of treated plants grouped together. On day 4 of harvest, we saw a separation between the control samples and the volatile exposed samples. On day 7, control samples and samples exposed to volatiles grouped together. These results were consistent with the previous result showing that $L R$ density increased after 2 days of exposure to $L$. bicolor volatiles 
(chapter 3.4.2). Together these findings indicate that volatiles associated genes were activated on the second day of treatment with fungal volatiles.

We used annotated count tables to calculate log-fold changes of volatile exposed samples compared to control samples. Genes whose transcript abundances differed significantly $p_{\text {adjusted }} \leq 0.05$ were considered as differentially expressed genes (DEGs). Across all time points, forty-four DEGs were found (Table 18; Table S3). Fourteen DEGs were upregulated and 30 DEGs were downregulated. We identified 42 DEGs in roots, which were exposed to volatiles for 2 days and only one DEG in roots that had been exposed for 4 or 7 days to volatiles. The gene At4g31330 was found on day 2, 4 and 7 of exposure to volatiles. The At4g31330 gene encodes a protein of unknown function with a domain of unknown function 599. We tentatively denominated this gene VALR (Volatile Affected Lateral Root). On all days of exposure to volatiles, VALR was significantly downregulated (Table 18).

The downregulation of VALR was corroborated by qRT-PCR (Figure 25) using cDNA synthesized from the same RNA as used for sequencing (chapter 2.10.2). VALR was downregulated over time in roots of volatile exposed plants as well as in roots of nonexposed plants. However, downregulation was significantly increased in volatile exposed roots. The difference was apparent after two days of co-incubation (Figure 25). 
Table 18. DEGs found in the transcriptome of $A$. thaliana roots at different times of exposure to volatiles of $L$. bicolor. The experimental set up is described in chapter 2.1.4 and harvest of the samples in chapter 2.9.1. $\log _{2}$ fold change (FC) and adjusted $p$ value were determined with DESeq2 package in $\mathrm{R}$.

\begin{tabular}{|c|c|c|c|c|}
\hline $\begin{array}{l}\text { days of } \\
\text { expo- } \\
\text { sure }\end{array}$ & Agi.ID & Name & $\log _{2} F C$ & $\begin{array}{l}\text { Adjusted } p \\
\text { value }\end{array}$ \\
\hline 0 & - & - & - & - \\
\hline 2 & At2g22990 & sinapoylglucose 1 & -0.797922 & $6.57 \mathrm{E}-03$ \\
\hline 2 & At2g22980 & serine carboxypeptidase-like 13 & -0.759635 & $1.19 \mathrm{E}-03$ \\
\hline 2 & At1g78290 & $\begin{array}{l}\text { Protein kinase superfamily } \\
\text { protein }\end{array}$ & -0.730835 & $3.94 \mathrm{E}-02$ \\
\hline 2 & At1g10070 & $\begin{array}{l}\text { branched-chain amino acid } \\
\text { transaminase } 2\end{array}$ & -0.710967 & 4.74E-02 \\
\hline 2 & At1g63180 & $\begin{array}{l}\text { UDP-D-glucose/UDP-D- } \\
\text { galactose 4-epimerase } 3\end{array}$ & -0.698748 & $3.94 \mathrm{E}-02$ \\
\hline 2 & At5g06690 & WCRKC thioredoxin 1 & -0.648129 & $4.21 \mathrm{E}-02$ \\
\hline 2 & At4g31330 & $\begin{array}{l}\text { Protein of unknown function, } \\
\text { DUF599 }\end{array}$ & -0.644963 & 6.37E-04 \\
\hline 2 & At1g66460 & $\begin{array}{l}\text { Protein kinase superfamily } \\
\text { protein }\end{array}$ & -0.624369 & 4.74E-02 \\
\hline 2 & At3g61060 & phloem protein $2-\mathrm{A} 13$ & -0.618872 & $1.72 \mathrm{E}-03$ \\
\hline 2 & At3g49780 & phytosulfokine 4 precursor & -0.604256 & $3.94 \mathrm{E}-02$ \\
\hline 2 & At1g80920 & $\begin{array}{l}\text { Chaperone DnaJ-domain } \\
\text { superfamily protein }\end{array}$ & -0.592743 & $2.28 \mathrm{E}-02$ \\
\hline 2 & At3g49790 & Carbohydrate-binding protein & -0.579636 & $4.44 \mathrm{E}-02$ \\
\hline 2 & At3g48360 & BTB and TAZ domain protein 2 & -0.572728 & $3.94 \mathrm{E}-02$ \\
\hline 2 & At5g54300 & $\begin{array}{l}\text { Protein of unknown function } \\
\text { DUF761 }\end{array}$ & -0.553514 & $2.28 \mathrm{E}-02$ \\
\hline 2 & At3g53980 & $\begin{array}{l}\text { Bifunctional inhibitor/lipid- } \\
\text { transfer protein/seed storage } 2 \mathrm{~S} \\
\text { albumin superfamily protein }\end{array}$ & -0.540325 & 3.94E-02 \\
\hline
\end{tabular}




\begin{tabular}{|c|c|c|c|c|}
\hline 2 & At1g73750 & $\begin{array}{l}\text { Uncharacterised conserved } \\
\text { protein UCP031088, alpha/beta } \\
\text { hydrolase }\end{array}$ & -0.518523 & $3.40 \mathrm{E}-02$ \\
\hline 2 & At1g18330 & $\begin{array}{l}\text { Homeodomain-like superfamily } \\
\text { protein }\end{array}$ & -0.500569 & $1.55 \mathrm{E}-02$ \\
\hline 2 & At3g10113 & $\begin{array}{l}\text { Homeodomain-like superfamily } \\
\text { protein }\end{array}$ & -0.478380 & $3.40 \mathrm{E}-02$ \\
\hline 2 & At3g06850 & $\begin{array}{l}\text { 2-oxoacid dehydrogenases } \\
\text { acyltransferase family protein }\end{array}$ & -0.477327 & $4.21 \mathrm{E}-02$ \\
\hline 2 & At5g27350 & $\begin{array}{l}\text { Major facilitator superfamily } \\
\text { protein }\end{array}$ & -0.469442 & $2.28 \mathrm{E}-02$ \\
\hline 2 & At2g39000 & $\begin{array}{l}\text { Acyl-CoA N-acyltransferases } \\
\text { (NAT) superfamily protein }\end{array}$ & -0.440948 & $3.94 \mathrm{E}-02$ \\
\hline 2 & At5g21170 & $\begin{array}{l}\text { 5'-AMP-activated protein kinase } \\
\text { beta-2 subunit protein }\end{array}$ & -0.428172 & $1.55 \mathrm{E}-02$ \\
\hline 2 & At5g66880 & $\begin{array}{l}\text { sucrose nonfermenting } 1 \text { (SNF1)- } \\
\text { related protein kinase } 2.3\end{array}$ & -0.382266 & $1.55 \mathrm{E}-02$ \\
\hline 2 & At3g05165 & $\begin{array}{l}\text { Major facilitator superfamily } \\
\text { protein }\end{array}$ & -0.360387 & $3.94 \mathrm{E}-02$ \\
\hline 2 & At2g15695 & $\begin{array}{l}\text { Protein of unknown function } \\
\text { DUF829, transmembrane } 53\end{array}$ & -0.342555 & $3.94 \mathrm{E}-02$ \\
\hline 2 & At5g47390 & $\begin{array}{l}\text { myb-like transcription factor } \\
\text { family protein }\end{array}$ & -0.299774 & $4.21 \mathrm{E}-02$ \\
\hline 2 & At5g40670 & $\begin{array}{l}\text { PQ-loop repeat family protein / } \\
\text { transmembrane family protein }\end{array}$ & -0.282556 & 6.57E-03 \\
\hline 2 & At2g01490 & $\begin{array}{l}\text { phytanoyl-CoA dioxygenase } \\
\text { (PhyH) family protein }\end{array}$ & -0.258577 & 4.74E-02 \\
\hline 2 & At3g13690 & $\begin{array}{l}\text { Protein kinase protein with } \\
\text { adenine nucleotide alpha } \\
\text { hydrolases-like domain }\end{array}$ & 0.282435 & $2.60 \mathrm{E}-02$ \\
\hline
\end{tabular}




\begin{tabular}{|c|c|c|c|c|}
\hline 2 & At5g67510 & $\begin{array}{l}\text { Translation protein SH3-like } \\
\text { family protein }\end{array}$ & 0.289650 & $4.56 \mathrm{E}-02$ \\
\hline 2 & At1g24280 & $\begin{array}{l}\text { glucose-6-phosphate } \\
\text { dehydrogenase } 3\end{array}$ & 0.330743 & $3.94 \mathrm{E}-02$ \\
\hline 2 & At1g67110 & $\begin{array}{l}\text { cytochrome P450, family } 735 \text {, } \\
\text { subfamily A, polypeptide } 2\end{array}$ & 0.628353 & 2.03E-03 \\
\hline 2 & At1g52660 & $\begin{array}{l}\text { P-loop containing nucleoside } \\
\text { triphosphate hydrolases } \\
\text { superfamily protein }\end{array}$ & 0.640655 & $4.55 \mathrm{E}-02$ \\
\hline 2 & At2g16060 & hemoglobin 1 & 0.653521 & $3.94 \mathrm{E}-02$ \\
\hline 2 & At3g10040 & $\begin{array}{l}\text { sequence-specific DNA binding } \\
\text { transcription factors }\end{array}$ & 0.664037 & $3.40 \mathrm{E}-02$ \\
\hline 2 & At1g68880 & basic leucine-zipper 8 & 0.747453 & $6.18 \mathrm{E}-06$ \\
\hline 2 & At2g17050 & $\begin{array}{l}\text { disease resistance protein (TIR- } \\
\text { NBS-LRR class), putative }\end{array}$ & 0.774193 & $3.40 \mathrm{E}-02$ \\
\hline 2 & At4g01970 & stachyose synthase & 0.807244 & $3.47 \mathrm{E}-02$ \\
\hline 2 & At3g02550 & $\begin{array}{l}\text { LOB domain-containing protein } \\
41\end{array}$ & 0.844594 & $2.03 \mathrm{E}-03$ \\
\hline 2 & At3g47770 & ABC2 homolog 5 & 0.901002 & $6.57 \mathrm{E}-03$ \\
\hline 2 & At5g15120 & $\begin{array}{l}\text { Protein of unknown function } \\
\text { DUF1637 }\end{array}$ & 0.935177 & 4.39E-03 \\
\hline 2 & At1g49860 & $\begin{array}{l}\text { glutathione S-transferase } \\
\text { (class phi) } 14\end{array}$ & 1.275637 & $4.24 \mathrm{E}-07$ \\
\hline 4 & At4g31330 & $\begin{array}{l}\text { Protein of unknown function, } \\
\text { DUF599 }\end{array}$ & -0.945469 & $1.68 \mathrm{E}-09$ \\
\hline 7 & At4g31330 & $\begin{array}{l}\text { Protein of unknown function, } \\
\text { DUF599 }\end{array}$ & -0.660173 & $2.45 \mathrm{E}-03$ \\
\hline
\end{tabular}




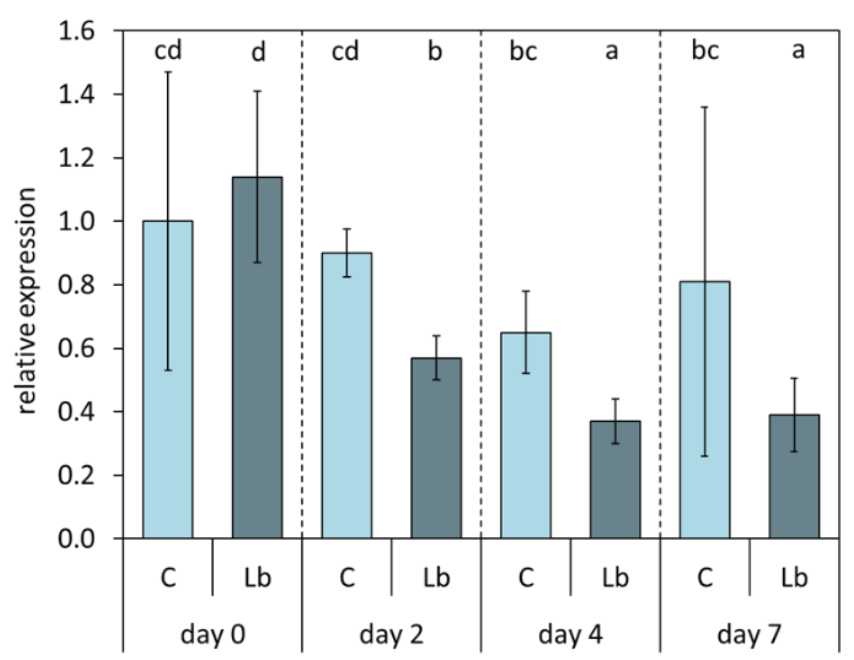

Figure 25. Relative normalized VALR expression in $A$. thaliana roots exposed to L. bicolor volatiles for 0, 2, 4 and 7 days. Harvest was conducted as described in chapter 2.9.1. qRT-PCR was performed with RNA samples from 3 compiled replicates which were also used for sequencing. For statistical analysis, an ANOVA with number of replicates as random factor was performed. Different letters above the bars indicate significant differences at $p<0.05$, according to post-hoc Tukey's test. C, control; Lb, L. bicolor

Besides the response to volatiles in whole roots, we tested, if the response to volatiles of $L$. bicolor differed between the root segment, in which LR were formed and the young elongation part from the tip onwards without visible LR emergence. We harvested these root sections after 2 days of volatile exposure and used them for RNAseq (Figure $27 \mathrm{~A}$ to $\mathrm{C}$ ). In addition, we analyzed whole roots to be able to compare the transcriptome of whole roots to those of harvested root segments. In additional replicates, we controlled that LR formation was stimulated in response to volatiles (Figure 26). 
A

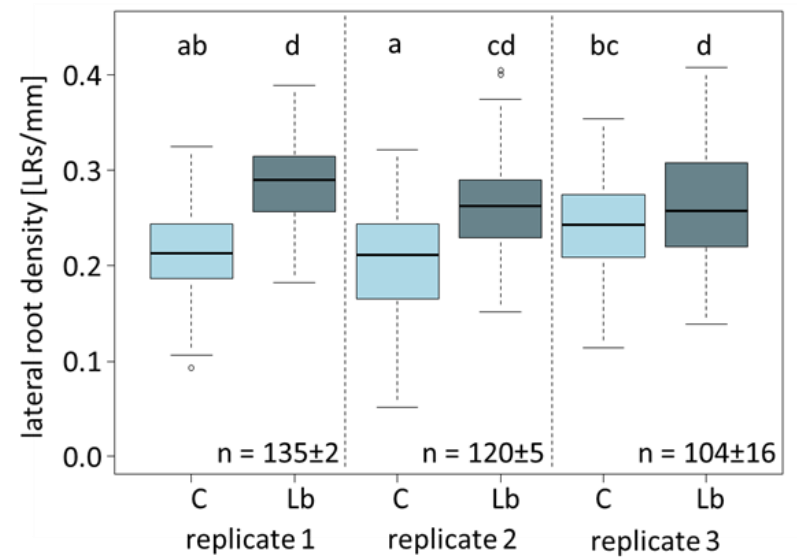

B

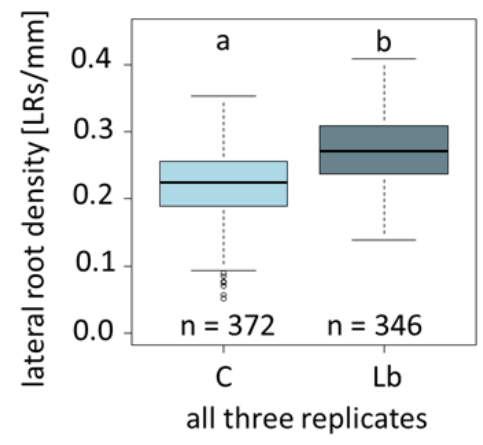

Figure 26. LR density of Arabidopsis wildtype plants grown together with samples for transcriptomic analysis of different root parts. A) LR density of each individual replicate. B) Data of all replicates were compiled. Four-day-old seedlings were exposed to volatiles of $L$. bicolor (14-day-old) for 7 days, according to the protocol in chapter 2.1.4. Ten petri dishes per treatment with 15 seeds per dish were prepared. Since not all seeds germinated the number of analyzed plants $(n)$ varied between treatments and replicates. Data originated from 3 independent replicates. For statistical analysis, an ANOVA with number of replicates as random factor was performed (in B). Different letters above the bars indicate significant differences at $p<0.001$, according to post-hoc Tukey's test. C, control; Lb, L. bicolor

PCA on transcriptome normalized count data revealed that samples were split according to the root segments along PC1 and according to volatile treatment along PC2 (Figure 27A). Log-fold changes and p-values for transcripts were determined as described in chapter 2.10.3. We found 5,859 DEGs in total. Among them, 7 were found in whole roots, 4873 were found in the LR part and 979 DEGs were found in the elongation-tip (ET) part (Figure 27B; Table S4). Five hundred and fifty overlapping DEGs were found in LR and ET part. One hundred and seventy-eight overlapping DEGs were downregulated and 371 were upregulated in both root parts (Figure 27C).

VALR was significantly downregulated in the LR part and elongation-tip part (log-fold change $-0.76 \pm 0.02, p<0.0001)$. Furthermore, VALR expression in the individual root parts was corroborated with qRT-PCR (Figure $28 \mathrm{~A}$ to C). Here, we found a significant lower expression in whole roots as well as in the LR- and elongation-tip part (Figure 28A). 
These findings suggested that downregulation of VALR might play a role in increased LR formation upon treatment with L. bicolor volatiles.

To get an overview on the cellular processes in which the DEGs were involved in, we assigned the DEGs to functional groups using MapMan and tested whether they were significantly enriched or depleted compared to the abundance of the genes in these categories in the whole reference map (Figure 29A) as described in chapter 2.10.4. Significantly higher numbers were present in the MapMan categories Photosystem for both the LR- and the elongation-tip part, and protein (activation, synthesis, targeting, degradation, and posttranslational modification), RNA (processing and regulation of transcription), cell wall, microRNA, mitochondrial electron transport and glycolysis for the LR part (Figure 29).

Since protein and cell wall related genes can be associated with biotic stress (Dreher and Callis, 2007; Frugis and Chua, 2002; Houston et al., 2016), we had a closer look on DEGs mapped to the biotic stress MapMan category to see whether those DEGs were involved in the response to volatiles (Figure 29B). In consensus with the number of DEGs, which we found for the lateral-root or the elongation tip part, higher number of DEGs of the LR part were mapped to the Arabidopsis reference map (Ath_AGI_Locus_TAIR_Aug2012). In the lateral-root part, most of the DEGs were assigned to Proteolysis and Signaling categories, which are putatively involved in biotic stress. Most of the DEGs associated with proteolysis were downregulated. In signaling, most DEGs were upregulated. We also found DEGs, which were associated with Cell Wall and Abiotic Stress categories.

For the elongation-tip part, less DEGs were associated to proteolysis, signaling, abiotic stress or cell wall (Figure 29B). 
A

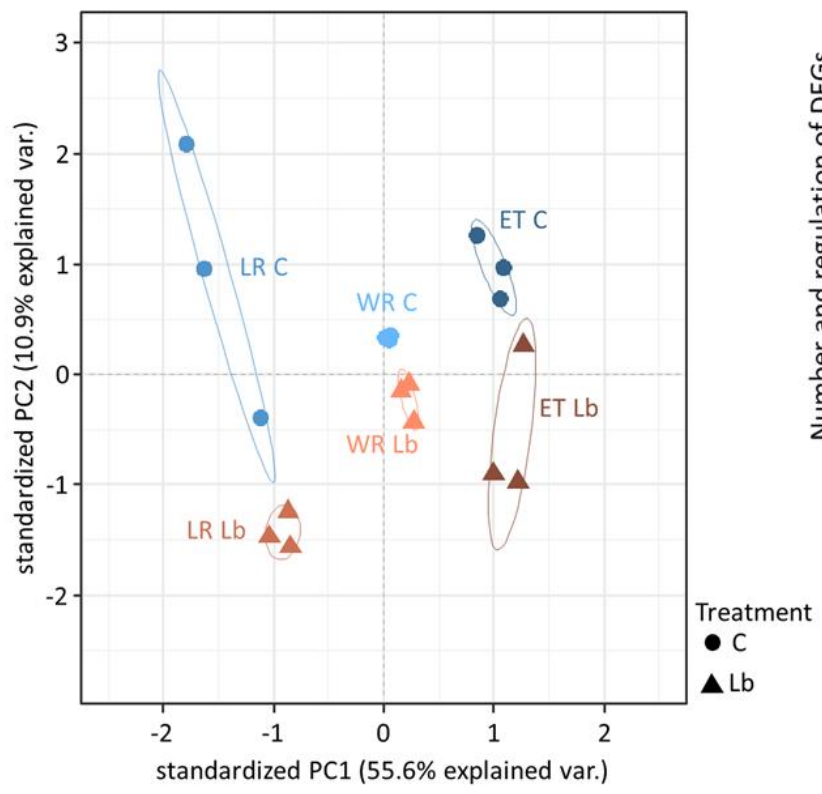

B

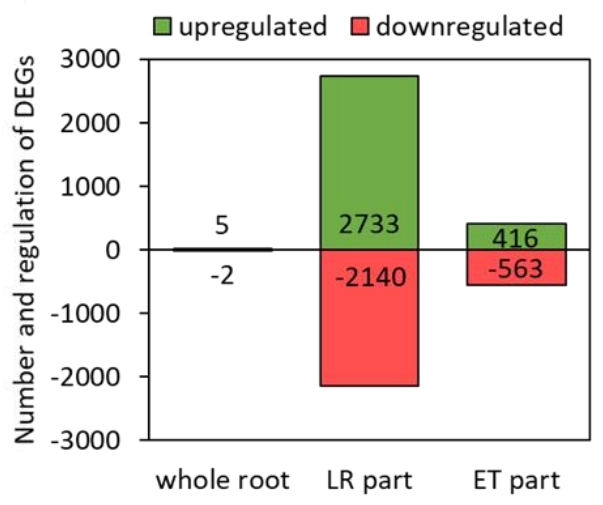

C

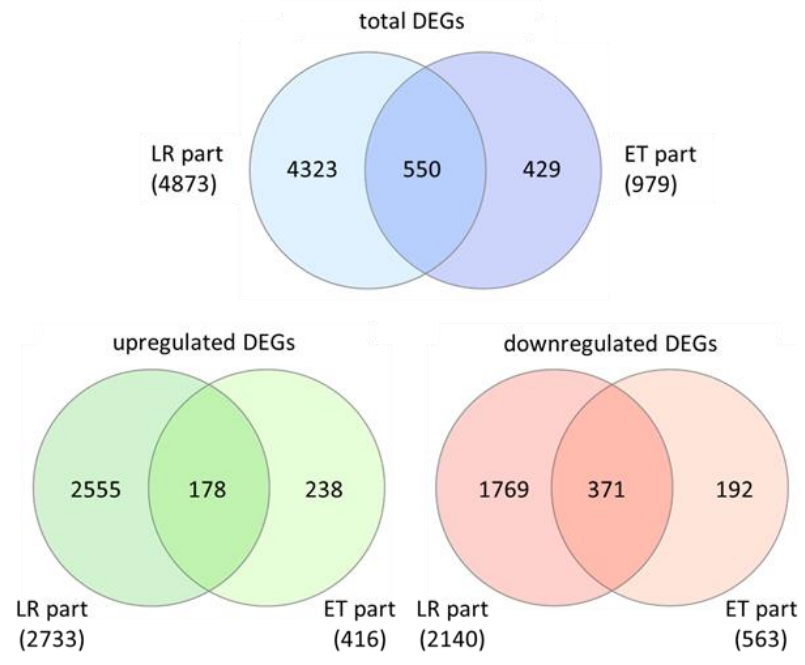

Figure 27. Transcriptome data from A. thaliana root parts A) PCA of transcriptomic normalized count data. RNA of the lateral root part and the elongation-tip part of A. thaliana roots were harvested after 2 days of exposure to volatiles of L. bicolor (chapter 2.1.4, 2.9.1). PC1 explains separation by root part and PC2 separation by treatment. This dataset included 19,274 transcripts. B) Number of DEGs found in the different root parts while comparing treated and non-treated samples. Upregulated DEGs according to log-fold change are shown in green and downregulated DEGs in red. C) Overlap of DEGs in the LR part and ET part of roots. DEGs were defined as genes with adjusted $p$ values $p<0.05$. C, control; Lb, L. bicolor; LR, lateral root part; $W R$, whole roots; $E T$, elongation-tip part 
A

B

C
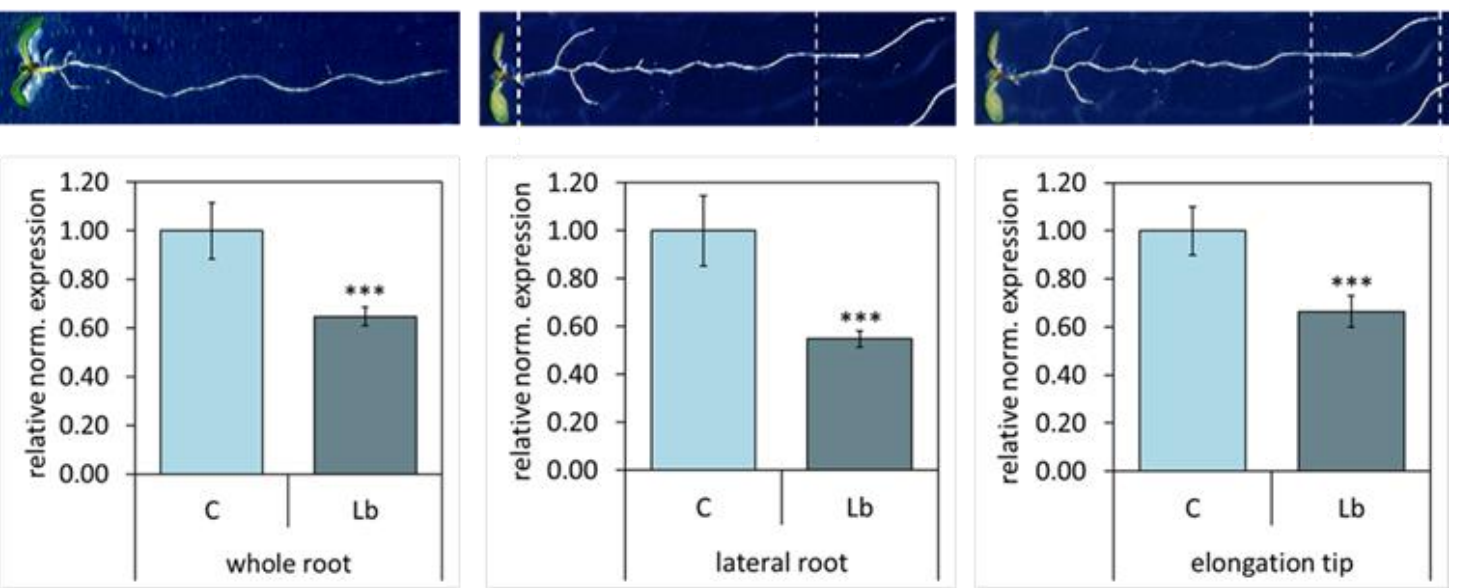

Figure 28. Relative normalized VALR expression in different roots parts of $A$. thaliana after 2 days of volatile exposure. Harvest was conducted as described in chapter 2.9.1. qRT-PCR was performed with RNA samples from 3 compiled replicates which were also used for sequencing. A) VALR expression in whole roots, B) VALR expression in the lateral root part, C) VALR expression in elongation-tip part. For statistical analysis, an ANOVA with number of replicates as random factor was performed. Asterisks indicate significant differences at $\mathrm{p}<0.001$. 
A

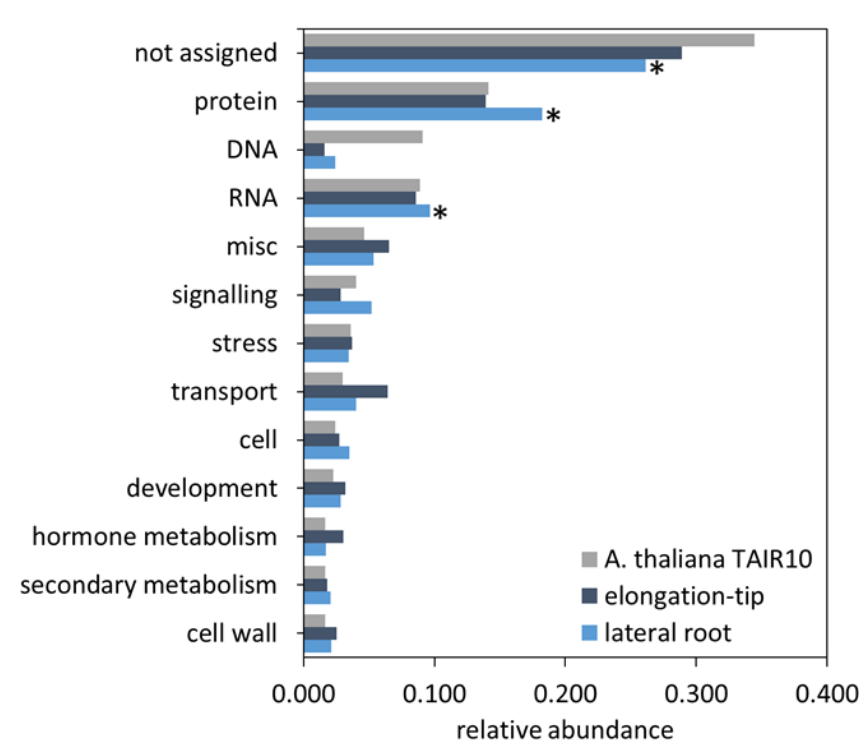

B

lipid metabolism micro RNA, natural antisense etc

amino acid metabolism

photosystem

redox

nucleotide metabolism

minor $\mathrm{CHO}$ metabolism

mitochondrial electron transport / ATP

major $\mathrm{CHO}$ metabolism

TCA / org transformation

Co-factor and vitamine metabolism

glycolysis

metal handling

tetrapyrrole synthesis

C1-metabolism

oxidative pentose phosphate pathways

$\mathrm{N}$-metabolism

Biodegradation of Xenobiotics

polyamine metabolism

fermentation

S-assimilation

gluconeogenesis / glyoxylate cycle

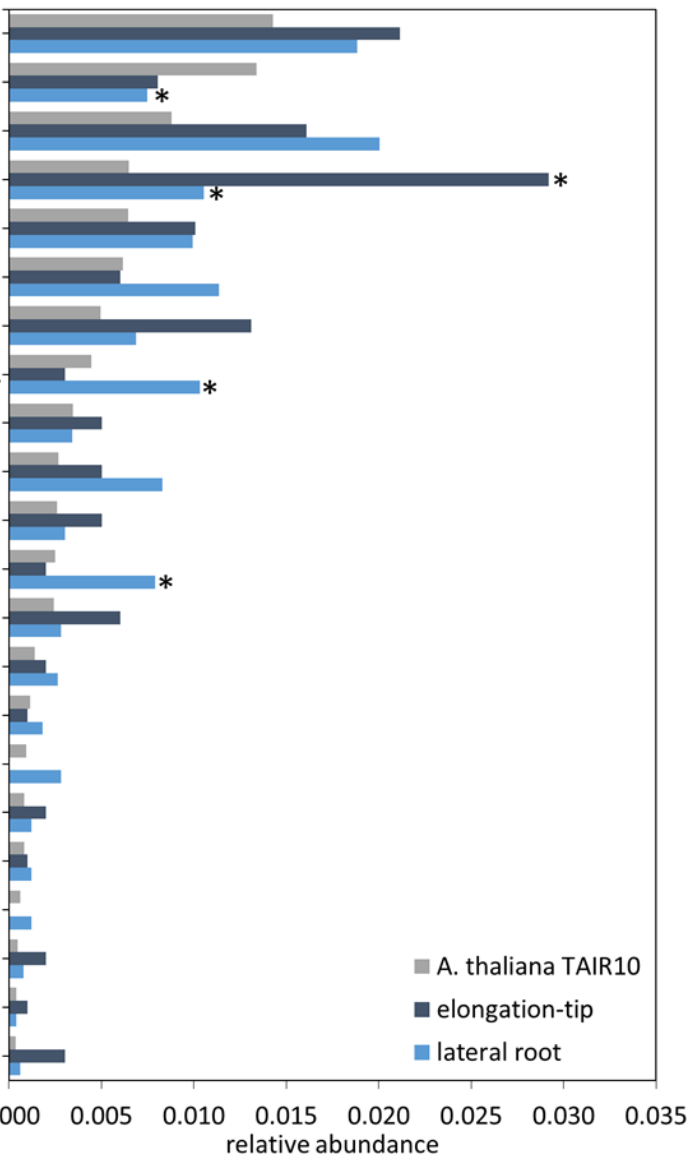

Figure 29. Assignment of differentially expressed genes in Arabidopsis root parts to MapMan categories. Relative abundance of DEGs in cellular processes compared to all genes involved in the reference $A$. thaliana genome. Figure was split into higher abundances (A) and lower abundances (B). Asterisks indicate significant different abundances compared to the reference map at $p<0.05$ according to Wilcoxon rank sum test and Benjamini Hochberg correction. 
A lateral root part

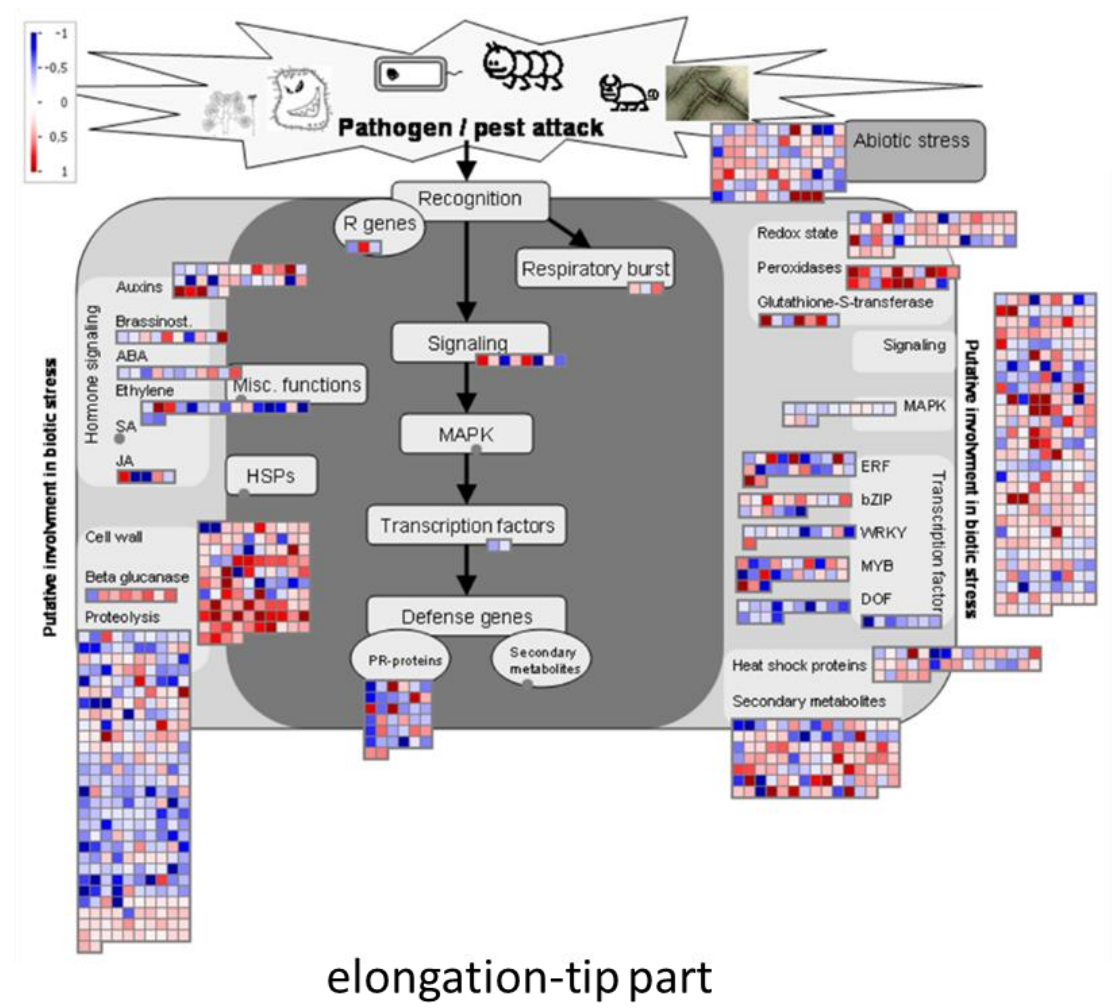

B

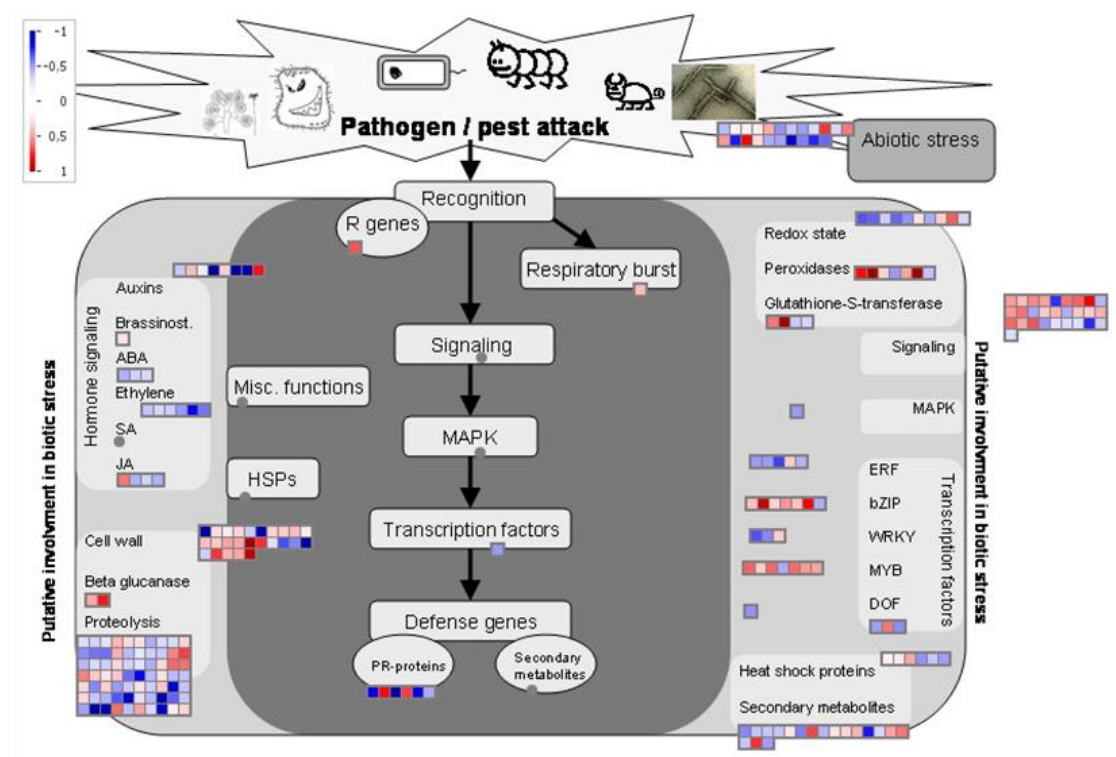

Figure 30. DEGs of $A$. thaliana root parts assigned biotic and abiotic stress in MapMan. DEGs found in either the lateral root part (A) or the elongation-tip part (B). $\log _{2}$ foldchanges of DEGs found in roots parts exposed to L. bicolor volatiles for 2 days were load into MapMan (chapter 2.10.4). Blue: downregulated DEGs; red: upregulated DEGs; dark grey panel: orthologous with genes having experimental indication of involvement with biotic stress responses; light grey panels: orthologous with genes that are putatively associated with biotic stress. 


\subsubsection{Transcriptional changes A. thaliana rosettes during exposure to L. bicolor volatiles for one week}

Besides the transcriptome of the roots, we investigated the transcriptome of rosettes which we harvested together with the roots in the time-course experiment at day 4. We did not identify any DEG (Table S5). This result suggests either that the response to volatiles does not affect the shoot transcriptome or that four days of exposure were too early or too late to observe differences.

\subsubsection{Metabolome analysis of $A$. thaliana roots and rosettes}

To obtain further information on the processes stimulated by volatile exposure, we investigated the metabolomes of Arabidopsis roots and rosettes exposed to volatiles of L. bicolor for 0, 2, 4 and 7 days by UHPLC-qTof-MS (Table S6). We performed PCAs based on normalized intensities of the "metabolites". It should be noted that "metabolites" were determined as cluster of compounds and distinguished by retention time ( $\mathrm{rt}$ ) and mass-to-charge ratio $(\mathrm{m} / \mathrm{z})$. In the following the term "metabolite" will be used. A PCA containing the whole metabolite data set showed that root samples exhibited much stronger scattering than rosette samples and both tissues were clearly separated (Figure 31A). We also analyzed the tissues separately. For rosettes, we detected a separation according to the time of exposure to volatiles (PC1), but no treatment effect (Figure 31B). The root metabolomes showed a clear time-dependent separation (Figure 31C). The effect of fungal volatile exposure resulted in no clear separation of the control and treated samples (Figure 31C). 

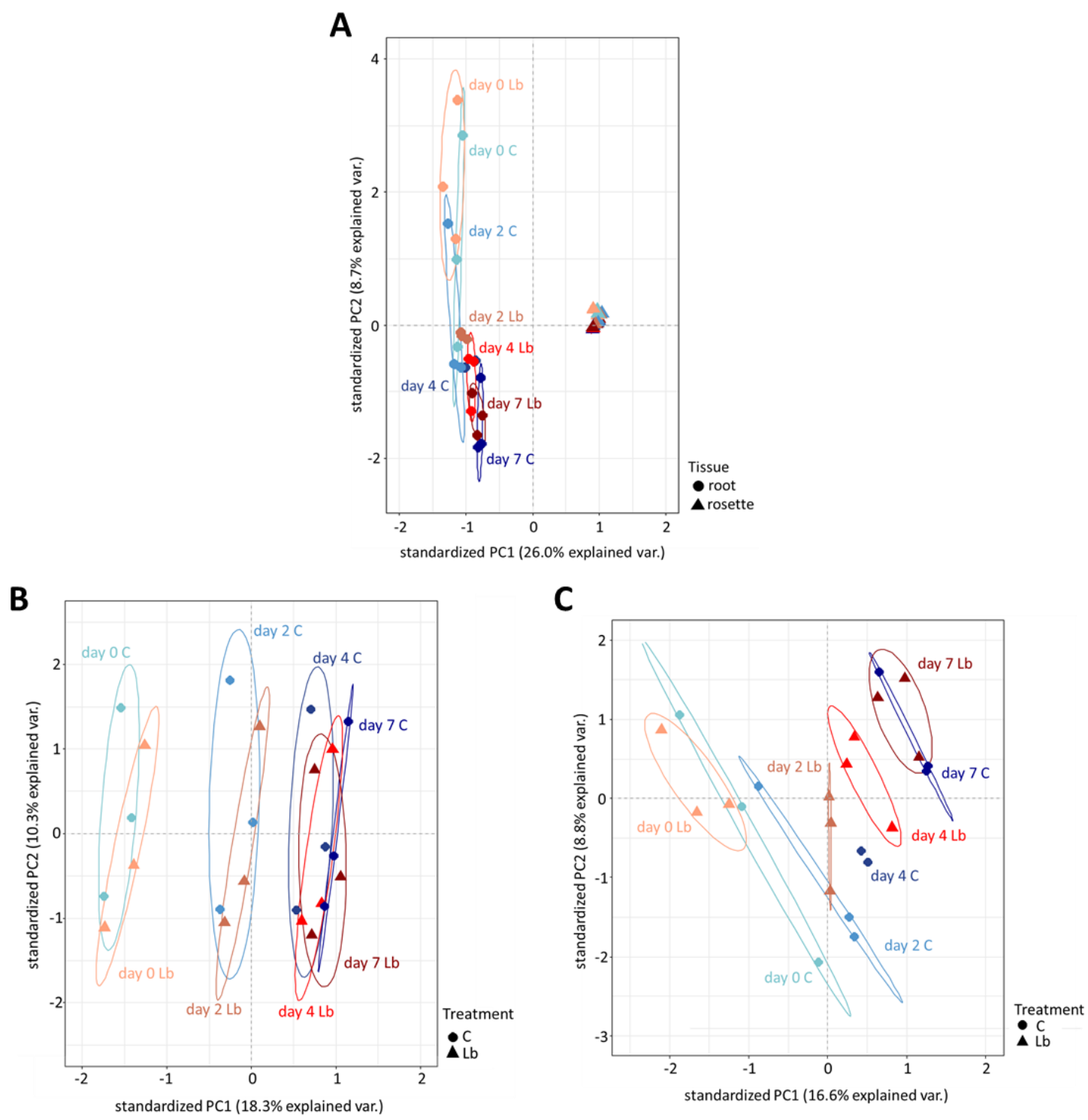

Figure 31. PCA of normalized metabolome intensity data of A. thaliana seedlings exposed to volatiles A) PCA of metabolome data from roots and rosettes. PC1 explains separation by tissue and PC2 by time. B) PCA of rosette data. PC1 explains separation by time and PC2 by treatment. C) PCA of root data. PC1 explains separation by time and PC2 by treatment. During processing, one of the 4 days exposure control sample was lost. Thus, only 2 data points remained. Four 4-day-old seedlings were exposed to L. bicolor volatiles for $0,2,4$, and 7 days. Plants for metabolite analysis were grown as described in chapter 2.1.4 and harvested as described in chapter 2.9.1. During processing, one of the 4 days exposure control sample was lost. Thus, only 2 data points remained for sequencing. C, control; Lb; L. bicolor 
By comparing data from treated and non-treated samples, only one significantly regulated cluster in negative mode was found in rosettes exposed to volatiles for 2 days (Cluster_04691_n, $p=0.047)$. This cluster was annotated with 7 putative metabolites in the HMDB (Human Metabolome Database): aldosterone, cortisone, 19-oic-deoxycorticosterone, prednisolone, tricyclodehydroisohumulone, and 7-methylrosmanol. None of them was associated with any metabolome pathway in Arabidopsis according to Pathway Analysis in MetaboAnalyst 4.0 (https://www.metaboanalyst.ca/MetaboAnalyst/ModuleView.xhtml). Further searches in the literature showed that none of the mentioned metabolites occur in Arabidopsis. Since the PCA for rosettes and roots showed differences during the incubation time, we identified the underlying differentially abundant metabolites clusters (Figure 32A; Table S8). Under control conditions, the highest number of regulated clusters were found in comparison of day 0 , day 4 and day 7 (Figure 32A). Under L. bicolor volatile exposure, the highest number of regulated clusters was found between day 0 and day 7 (Figure 32A). We analyzed these data in a Venn-diagram (Figure 32B). Thereby, we found 2061 clusters in Arabidopsis rosettes grown under control conditions and 40 cluster in rosettes exposed to volatiles (Figure 32B).

We used MetaboAnalyst 4.0

(https://www.metaboanalyst.ca/MetaboAnalyst/ModuleView.xhtml) to assign the regulated and annotated clusters found in rosettes grown under control conditions, to Arabidopsis pathways as described in chapter 2.10.12 (Figure 33). In general, only a few hits per metabolic pathways were identified and the metabolites were not significantly enriched in any pathway. In rosettes grown under control condition for 4 days the number hits for the arachidonic acid metabolism, nitrogen metabolism, alanine, aspartate and glutamate metabolism, linoleic acid metabolism, arginine and proline metabolism, arginine biosynthesis, stilbenoid, diarylheptanoid and gingerol biosynthesis, and flavone and flavonol biosynthesis were higher than the expected hit number (according to MetaboAnalyst 4.0) (Figure 33A). In rosettes grown under control condition for 7 days the number of hits for sphingolipid metabolism, linoleic acid metabolism, galactose metabolism, and flavone and flavonol biosynthesis were higher than the expected hit number (according to MetaboAnalyst 4.0) (Figure 33B). 
Among the 40 clusters found in rosettes exposed to volatiles at day 7, 7 were annotated.

The MetaboAnalyst4.0 found 2 putative hits, one in riboflavin metabolism and one in purine metabolism.

A

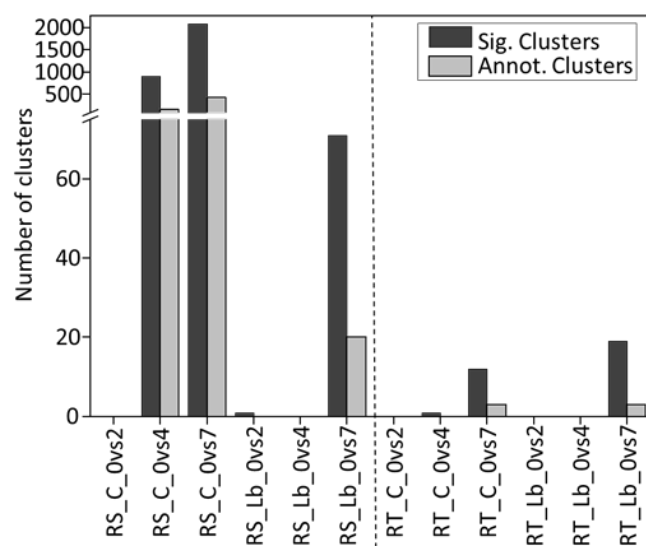

\begin{tabular}{|l|l|c|c|c|c|}
\hline $\begin{array}{l}\text { Plant } \\
\text { tissue }\end{array}$ & Condition & $\begin{array}{l}\text { Compared } \\
\text { days of } \\
\text { exposure }\end{array}$ & $\begin{array}{l}\text { Number of } \\
\text { singificant } \\
\text { clusters }\end{array}$ & $\begin{array}{l}\text { Number of } \\
\text { annotated } \\
\text { cluster }\end{array}$ & $\begin{array}{l}\text { lercentage } \\
\text { of } \\
\text { annotated } \\
\text { cluster }\end{array}$ \\
\hline rosette & control & 0 vs. 2 & 0 & 0 & $0 \%$ \\
\hline rosette & control & 0 vs. 4 & 900 & 157 & $17 \%$ \\
\hline rosette & control & 0 vs. 7 & 2092 & 435 & $21 \%$ \\
\hline rosette & L. bicolor & 0 vs. 2 & 1 & 0 & $0 \%$ \\
\hline rosette & L. bicolor & 0 vs. 4 & 0 & 0 & $0 \%$ \\
\hline rosette & L. bicolor & 0 vs. 7 & 71 & 20 & $28 \%$ \\
\hline root & control & 0 vs. 2 & 0 & 0 & $0 \%$ \\
\hline root & control & 0 vs. 4 & 1 & 0 & $0 \%$ \\
\hline root & control & 0 vs. 7 & 12 & 3 & $25 \%$ \\
\hline root & L. bicolor & 0 vs. 7 & 0 & 0 & $0 \%$ \\
\hline root & L. bicolor & 0 vs. 4 & 0 & 0 & $0 \%$ \\
\hline root & L. bicolor & 0 vs. 7 & 19 & 3 & $16 \%$ \\
\hline
\end{tabular}

B

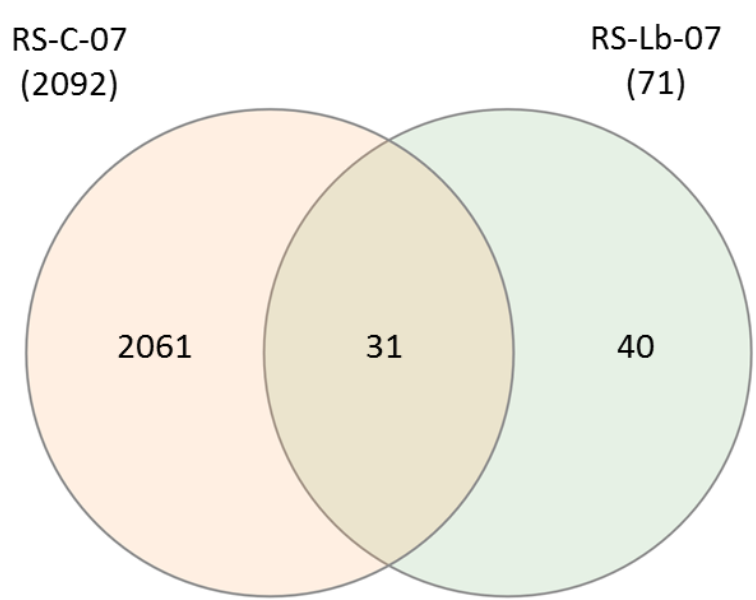

Figure 32. Number of clusters found in metabolome analysis of $A$. thaliana in day-wise comparison. A) Clusters were found either in rosettes (RS) or roots (RT) under control conditions (C) or treatment with volatiles of $L$. bicolor (Lb). Shown are only the comparisons between day 0 and day 2, 4 and 7. The table shows the exact data. B) Venn diagram of found significant regulated clusters in untreated (C) or treated ( $L b$ ) rosettes of day 7 compared to day 0 . Harvest of samples was performed as described in chapter 2.9.1. 
A

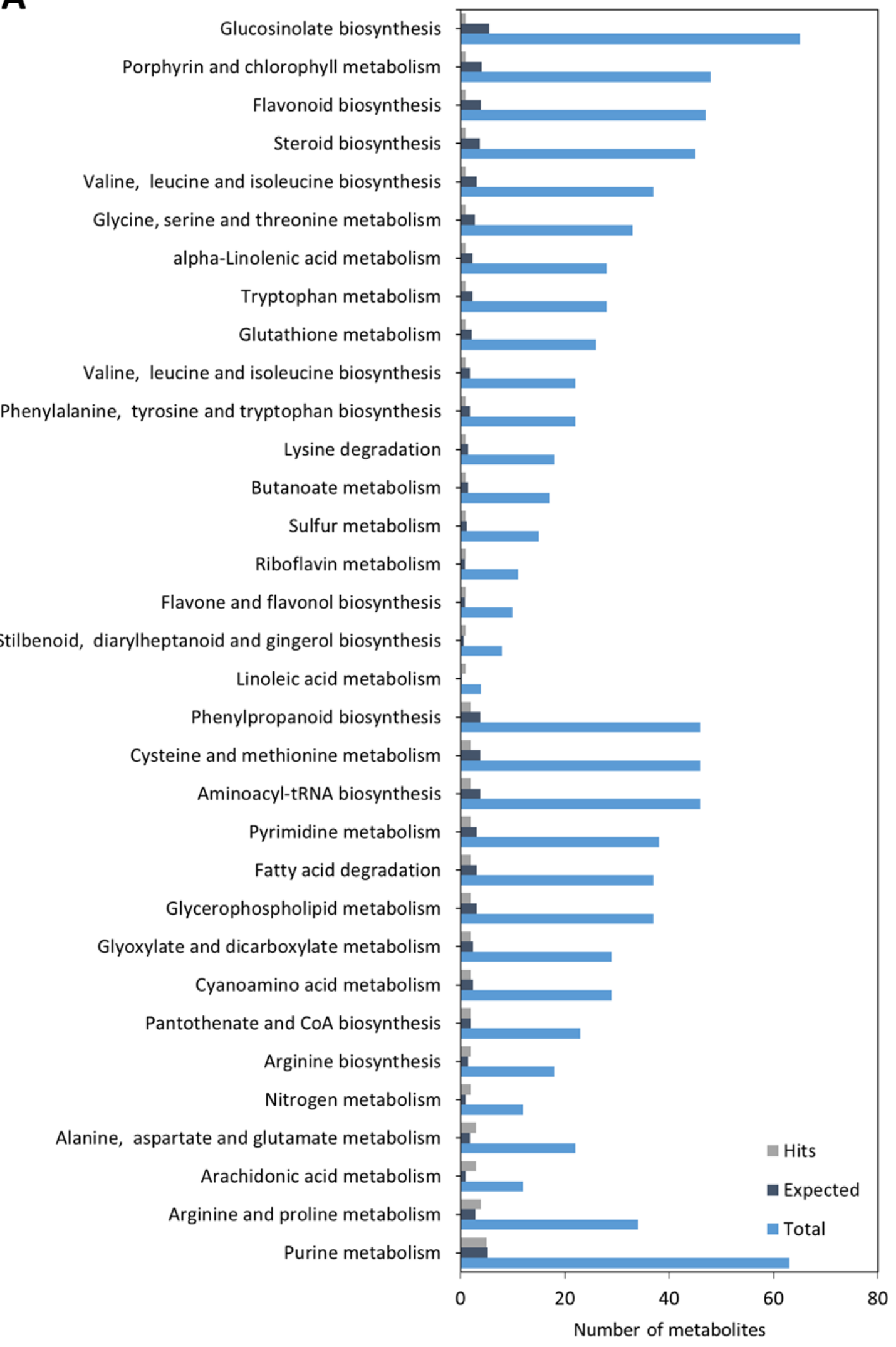

Figure 33A. Legend on page 124. 
B

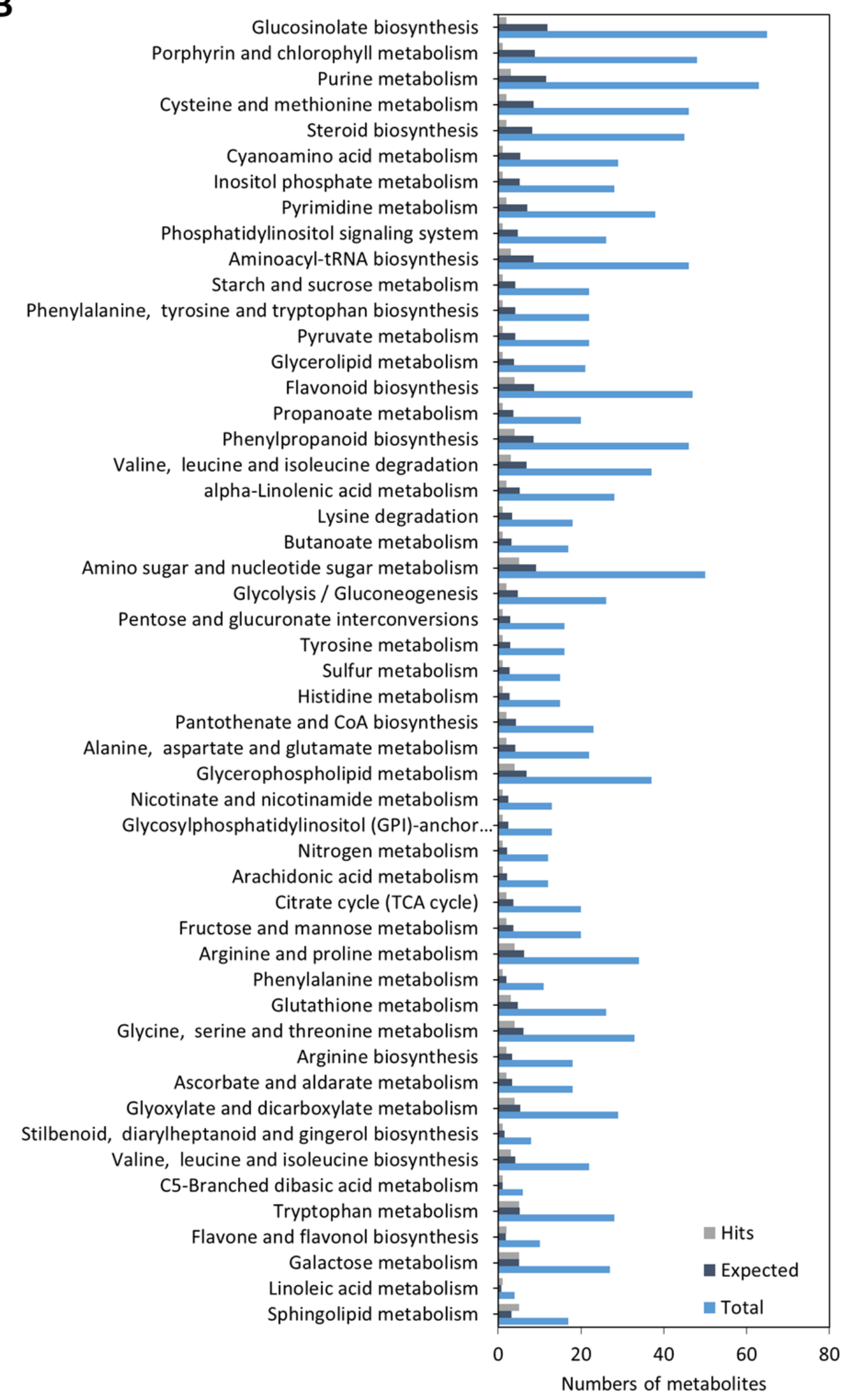

Figure 33B. Legend on next page. 
Figure 33. Putative metabolites of $A$. thaliana assigned to metabolic processes. HMDB IDs were used to annotate significant regulated cluster in $A)$ rosettes under control condition 4 days after experimental start and B) rosettes under control conditions after 7 days of experimental start. Data were compared to metabolites found at 0 of the experiment in 4-day-old plants. HMDB IDs were load in the Pathway Analysis of MetaboAnalyst (https://www.metaboanalyst.ca/MetaboAnalyst/ModuleView.xhtml) to identify putative metabolome pathways.

\subsubsection{Transcriptional changes in P. $x$ canescens roots exposed to $L$. bicolor volatiles for different time periods}

Besides the $A$. thaliana transcriptome, the root transcriptome of the L. bicolor host P. x canescens was examined. For this purpose, 14-day-old poplars were exposed to L. bicolor volatiles in bi-compartmented petri dishes for 0, 2, 4, 7 and 14 days. We analyzed the transcriptome of roots, which were exposed to volatiles for 2 and 7 days (Figure 34A). PCA revealed one outlier (day 2, control) (Figure 34A). The other data points showed separation by time of exposure along PC1 and by volatile treatment along PC2. In total, we found 921 DEGs in roots exposed to volatiles of L. bicolor for 3 days. Ninety-four of them were upregulated, 827 downregulated (Figure 34; Table S7). In roots, which were exposed to volatiles for 7 days, 271 DEGs were identified. One hundred and eighty-six were upregulated and 85 downregulated (Figure 34B). MapMan was used to assign the Potri.IDs to functional groups as described in chapter 2.10.4. In roots, which were exposed to volatiles for 2 days, we detected a significant number of genes in the not assigned category and the Cell Wall category (Figure 34C).

Along with the poplar plants, which were harvested for RNAseq, we determined primary root length and the LR density (Figure 35). After 2 days of exposure to volatiles, the LR density was increased significantly compared to roots without volatile treatment (Figure 35). Overall, LR density increased stronger in volatile exposed plants than in controls during the time course of 14 days (Figure 35). These results corroborate that fungal volatile expose induced enhanced LR formation after about 2 days. 
A

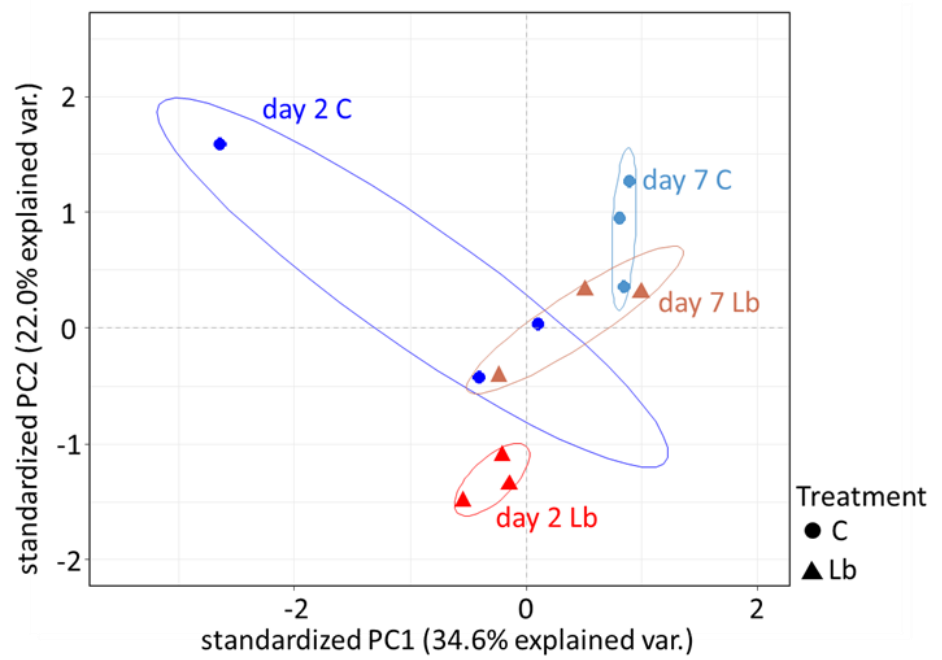

B

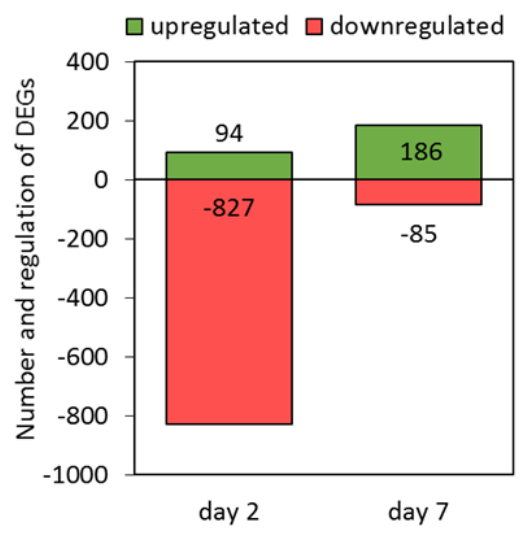

\section{C}

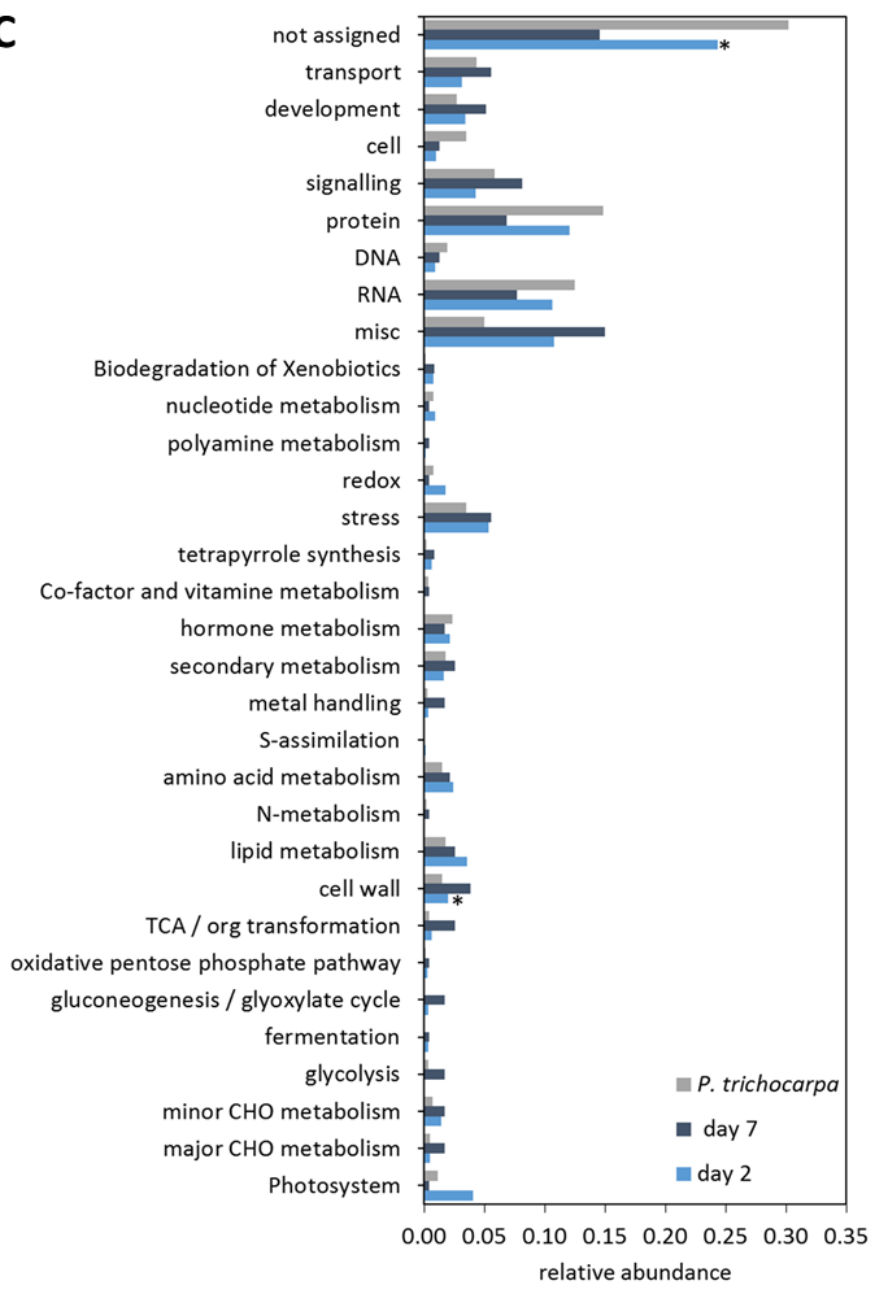

Figure 34. Legend on next page. 
Figure 34. Transcriptome of poplar roots exposed to L. bicolor volatiles. A) PCA of normalized count data. PC1 explains separation by time and PC2 by treatment. This dataset included 31,537 transcripts; C, control; Lb, L. bicolor B) Number and regulation of found DEGs. C) MapMan analysis of transcriptomic data. Relative abundance of DEGs in cellular processes compared to all genes involved in the reference $P$. trichocarpa genome. Asterisks indicate significant different abundances compared to the reference genome at $p<0.05$ according to Wilcoxon rank sum test and Benjamini Hochberg correction. RNA of roots exposed to volatiles for 2 or 7 days was sequenced. For RNA extraction roots of 4 poplars per day of exposure and treatment were pooled. Poplars grew in one petri dish each. Data originated from 3 independent experiments. All genes with $p<0.05$ were considered as DEGs. Number of upregulated genes are shown in green and number of downregulated genes in red.

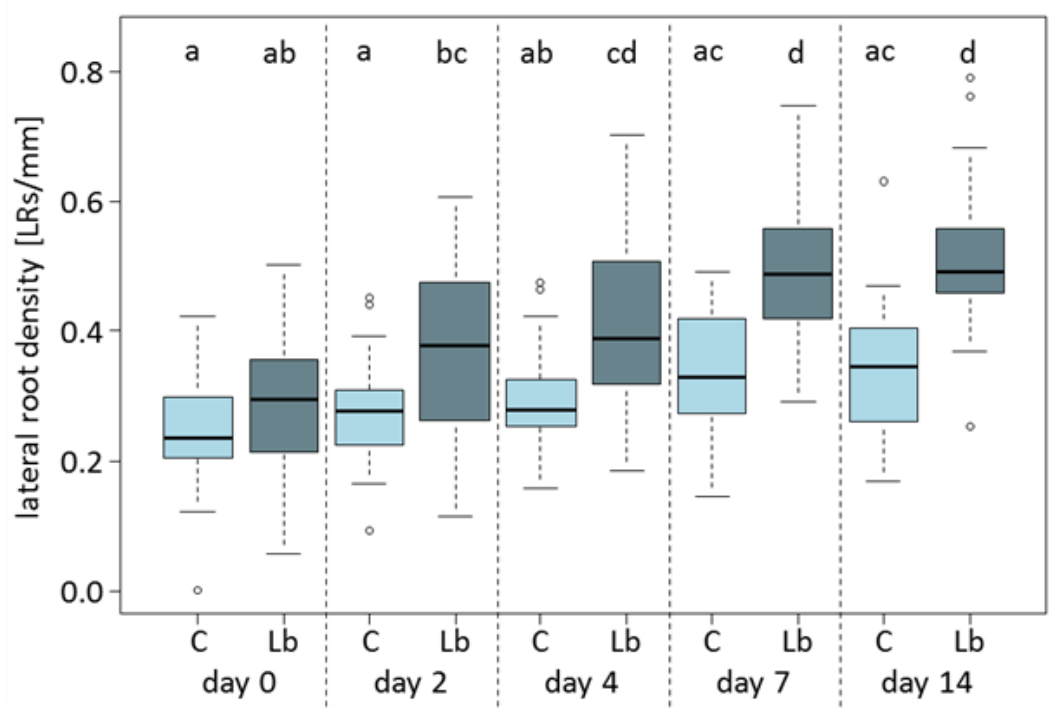

Figure 35. LR density of poplar roots during a time-course experiment. Plants were exposed to L. bicolor (12-day-old) volatiles for 0, 2, 4, 7 and 14 days. On each day, the same plants were observed. Seven to 10 plants grown in individual petri dishes were prepared per condition and replicate. The experiment was performed in 3 replicates. For statistical analysis, an ANOVA with replicates as random factor was performed. Number of experiments was treated as random factor. Different letters above the bars indicate significant differences at $\mathrm{p}<0.01$, according to post-hoc Tukey's test. C, control; Lb, L. bicolor

\subsubsection{Transcriptional changes in $P . x$ canescens leaves exposed to $L$. bicolor volatiles for different time periods}

In addition to the transcriptome of poplar roots, we investigated the transcriptome of leaves, harvested together with the roots after 2 and 7 days of co-incubation as well. 
After processing the RNAseq data, we performed a PCA (Figure 36A). PC2 explained separation by time of exposure. Separation of the different treatments at PC1 was not obvious. We identified 100 DEGs after 2 days of exposure to volatiles (Figure 36B; Table S8). Among those, 67 where upregulated and 33 were downregulated (Figure 36B). After 7 days of treatment, 191 DEGs were identified, of which 142 were upregulated and 49 were downregulated (Figure 36B). We grouped the DEGs into functional MapMan categories. Although the relative abundance of DEGs in most functional groups was higher compared to the relative abundance of genes in the P. trichocarpa reference genome, these increases were not significant in any of the MapMan categories (Figure 36C). 
A

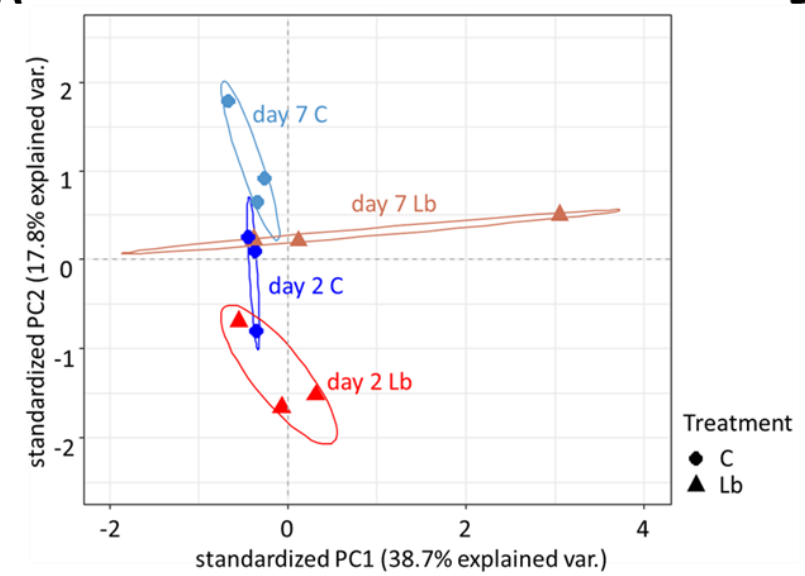

B

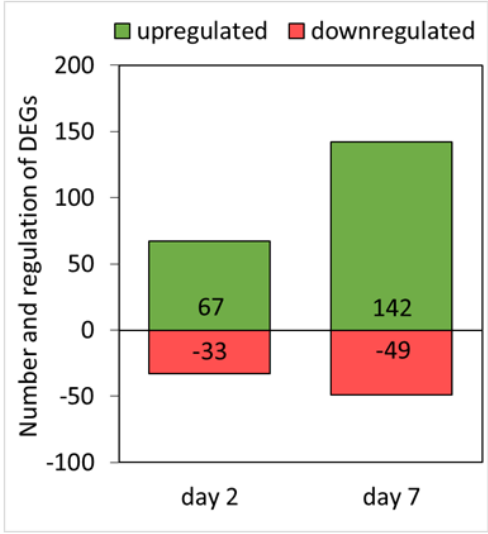

C

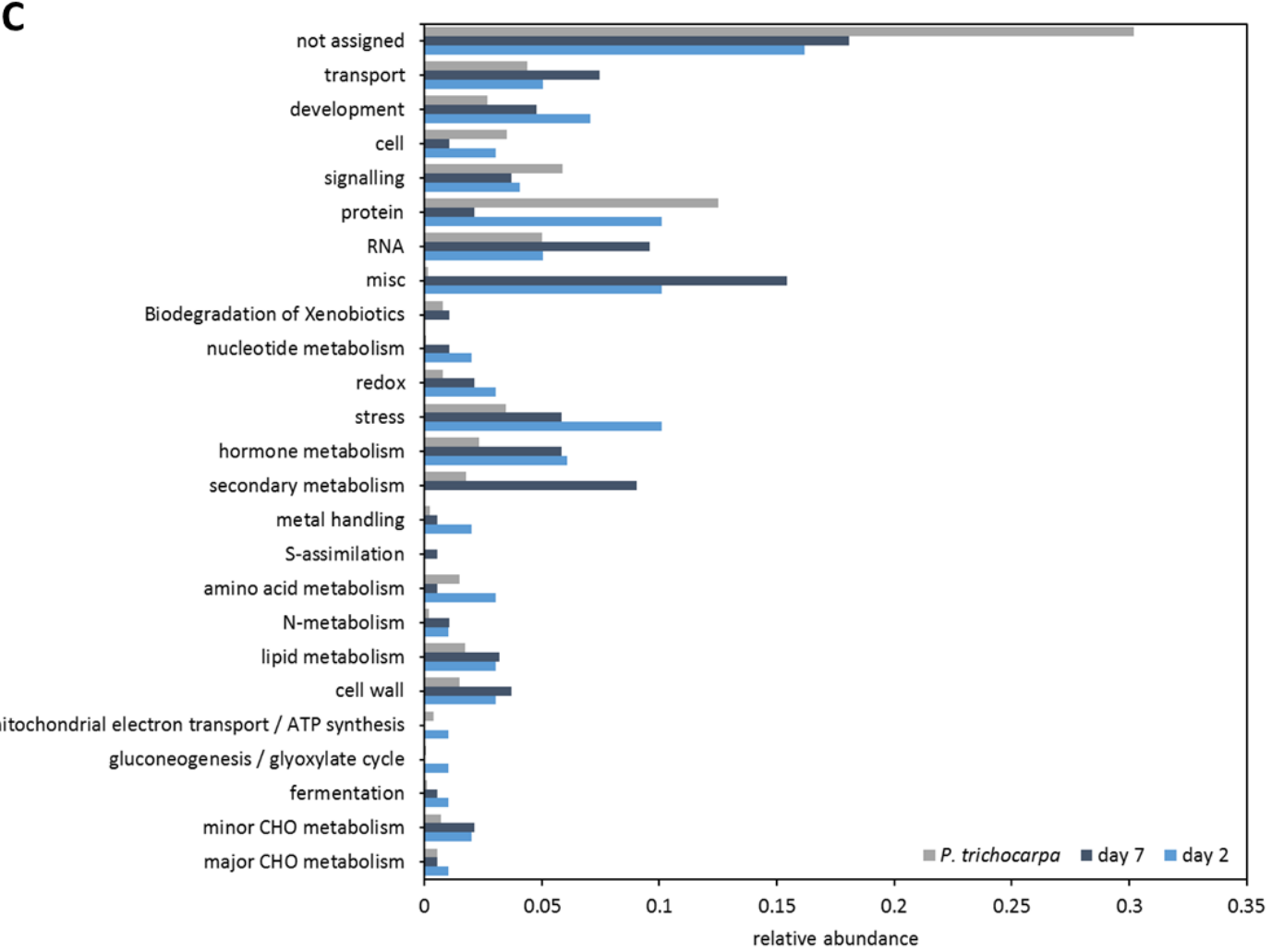

Figure 36. Transcriptome analysis of poplar leaves exposed to L. bicolor volatiles. A) PCA of normalized count data. PC1 explains separation by time and PC2 by treatment. This dataset included 25,195 transcripts. B) Number and regulation of found DEGs. RNA of leaves exposed to volatiles for 2 or 7 days was sequenced. Number of upregulated genes are shown in green and number of downregulated genes in red. For RNA extraction leaves of 4 poplars per day of exposure and treatment were pooled. Poplars grew in one petri dish each. Data originated from 4 independent replicates. All genes with $p<0.05$ were considered as DEGs. C, control; Lb, L. bicolor. C) MapMan analysis of transcriptomic data from poplar leaves exposed to volatiles for 2 or 7 days. Relative abundance of DEGs in cellular processes compared to relative abundance of genes of the reference P.trichocarpa genome. None of the cellular processes showed a significant different abundance compared to the reference genome. 


\subsubsection{Genes involved in hormone signaling pathways with effects on LR formation were found in transcriptome data}

Hormones, such as abscisic acid ( $A B A)$, auxin and ethylene are known to affect LR development (Fukaki and Tasaka, 2009, Waidmann et al., 2020) and therefore we searched for these genes in the transcriptome data sets of Arabidopsis and poplar. Furthermore, we searched for genes involved in jasmonic acid (JA) biosynthesis and signaling, since JA can influence LR formation as well (Raya-González et al., 2012; Sun et al., 2009). The genes included in our search are listed in Table S9.

In the Arabidopsis transcriptome data of root parts, $43 \%$ of seven JA-related genes, $40 \%$ of 15 ABA-related genes, $32 \%$ of 25 auxin-related genes and $21 \%$ of 14 ethylene related genes were differentially expressed (Figure 37A). However, in poplar $0 \%$ of $19 \mathrm{JA}$-related genes and $29 \%$ of 38 ABA-related genes were differentially expressed (Figure 37B). Five percent of 43 ethylene-related genes and $2 \%$ of 49 auxin-related genes were differentially expressed in poplar. The relatively strong enrichment in ABA-related genes suggested that this hormone might play a role in the LR response to volatiles of L. bicolor. In poplar, homologs of the Arabidopsis HAI (HIGHLY ABA-INDUCED) genes are downregulated. HAls are a members of the PP2C gene family (arabidopsis.org), which is involved in ABA signaling. Furthermore, in both, Arabidopsis and poplar roots, PYL and PYR genes were found, which are involved in ABA signaling as well. In Arabidopsis LR part, $A B A 1$ was downregulated, which plays role in $A B A$ biosynthesis.

As VALR (At4g31330) was significantly downregulated in Arabidopsis roots, we tested the regulation of the closest poplar homolog Potri.006G278000.1 with $74 \%$ amino acid identity (Figure 38). In consensus with the findings in A. thaliana roots, the poplar gene showed a negative fold change after 2 and 7 days of exposure to L. bicolor volatiles. However, this downregulation was not significant. 
A

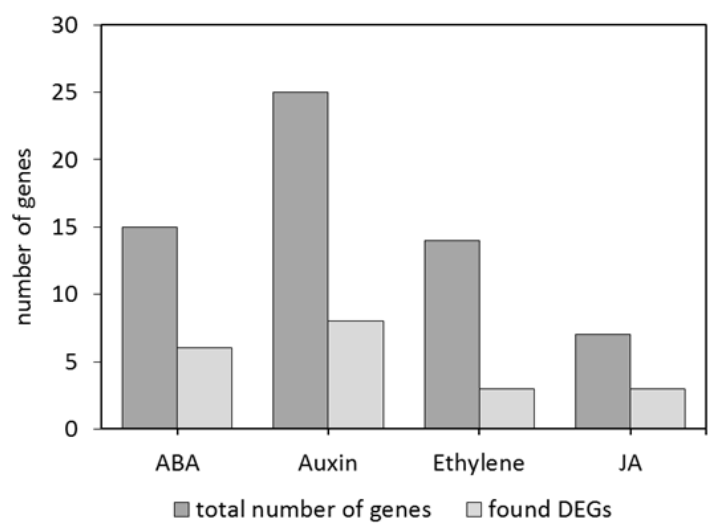

B

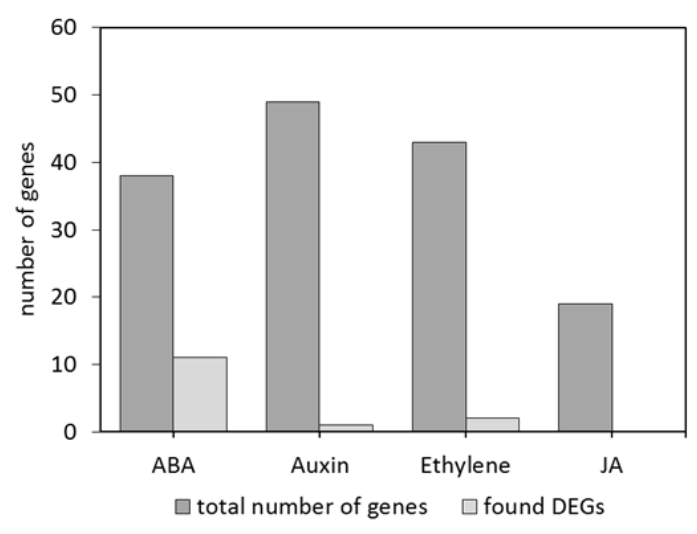

Figure 37. Number of hormone synthesis and signaling genes in transcriptome data of A. thaliana root parts (A) and P. $x$ canescens roots (B). We searched for abscisic acid $(A B A)$, auxin, ethylene and jasmonic acid (JA) synthesis or signaling related genes in the transcriptome data. The number of searched genes is presented in dark grey and the number of found DEGs in light grey. In poplar more genes are presented, since one Arabidopsis gene has more than one homolog in poplar. Data are shown in Table S9.

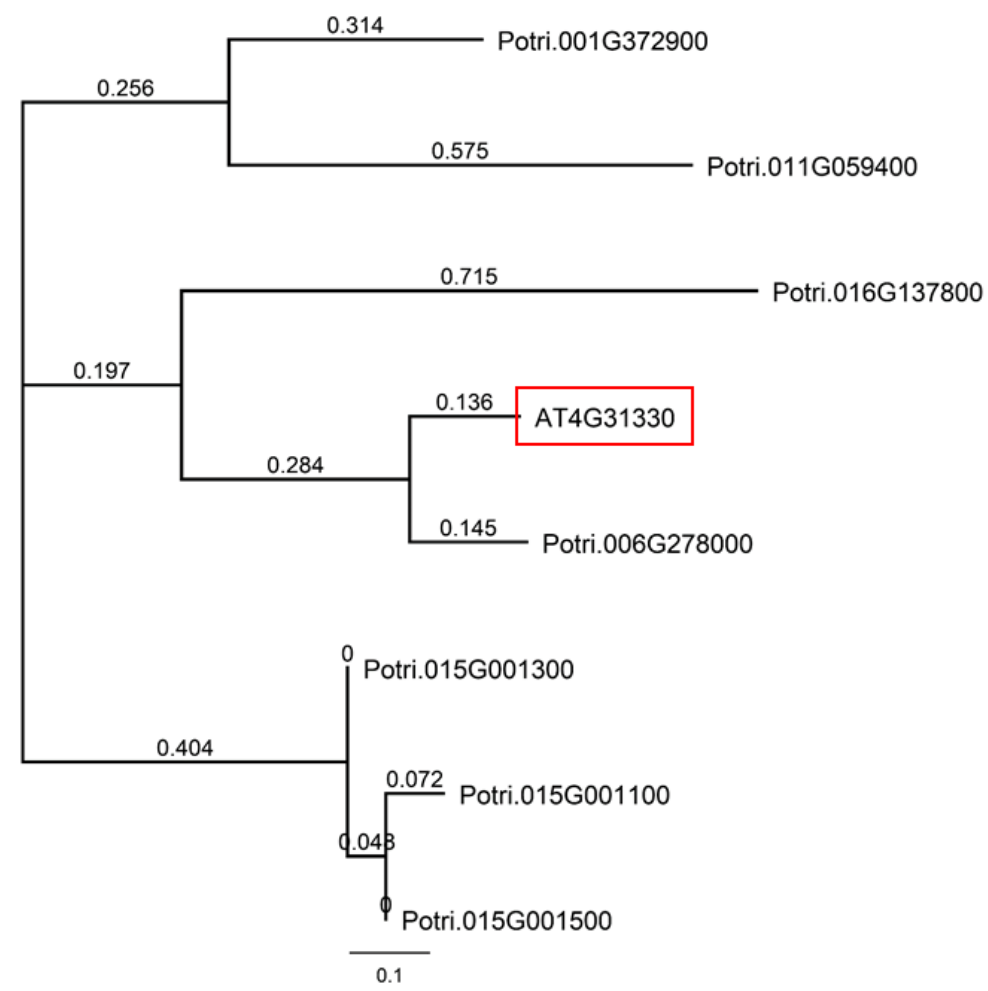

Figure 38. VALR (At4g31330) homologs in P. trichocarpa. Amino acid sequences were obtained from Phytozome 12 (JGI) and aligned in Geneious Prime as described in chapter 2.10.5 


\subsection{Screening candidate genes from transcriptome analyses for their involvement in volatile-induced LR formation}

\subsection{1 $A B A$ is suggested to be involved in volatile-induced LR formation}

Since our transcriptomic analyses of the effects of L. bicolor volatiles on poplar and Arabidopsis roots suggested a role of ABA in mediating enhanced LR, we tested loss-of function lines for ABA-related genes. We selected mutant lines that were either affected in ABA biosynthesis (aba2-1 or aao3-4) or in signaling (py/8, sextuple mutant pyr1 pyl1 pyl2 pyl4 pyl5 pyl8 and myb77-1) (Figure 39A). MYB77 was included because Zhao et al. (2014) suggested an alternative ABA signaling pathway involving this transcription factor during root growth recovery after salt stress induced quiescence (Zhao et al., 2014). Zhao et al. proposed that ABA activates PYL8, which directly interacts with MYB77 (Figure 39A). MYB77 forms a heterodimer with ARF7 leading to upregulation of auxin responsive genes, whose involvement in LR formation are known (Figure 39A; Shin et al., 2007; Zhao et al., 2014, Fukaki and Tasaka, 2009 and Waidmann et al., 2020).

Because ABA-related genes were mainly downregulated in the transcriptomic data of Arabidopsis and poplar roots (Table S9), we hypothesized a stronger LR formation in ABA synthesis loss-off function mutants upon exposure to volatiles of L. bicolor. In addition, we expected lower LR density in ABA synthesis loss-off function mutants under control conditions compared to wildtype. We also anticipated that the signaling mutant $p y / 8$ and the $p y r / p y /$ sextuple mutant caused lower LR densities under control conditions and strong LR formation in response to L. bicolor volatiles. In our transcriptomic data sets, myb77 was upregulated upon treatment with volatiles. Therefore, we expected lower LR formation in the loss-of function mutant. Four-day-old Arabidopsis mutant lines and wildtype (Col-0) seedlings were co-cultivated with 14-day-old L. bicolor for 7 days or without the fungus. Under control conditions, aba2-1 showed a significantly 1.15 -fold lower LR density than wildtype plants (Figure 39B). The LR density of aao3-4, the sextuple $p y r / p y l$ mutant and myb77-1 were also lower under control conditions than that of the wildtype. However, these reductions were not significant (Figure 39B).

All mutants showed enhanced LR densities upon treatment with volatiles. The LR density of line aba2-1 was 1.26 -fold increased while that of the wildtype was only 1.08 -fold 
increased $(p<0.001$, Figure 39B). The difference between volatile treated and non-treated roots of the sextuple $p y r / p y l$ mutant was also quite high (1.20-fold), although not significant compared to wildtype (Figure 39B). Aao3-4 and pyl8-1 showed no significant difference between treated and non-treated samples (Figure 39B). Thus, their response to volatiles was less intense compared to wildtype. Myb77-1 showed no difference compared to the wildtype (Figure 39B).

Hence, the ABA-synthesis mutant line aba2-1 showed an increased response to volatiles of L. bicolor compared to wildtype plants, as hypothesized. However, in contrast to our expectations, the response to L. bicolor volatiles in aao3-4 and py/8-1 was smaller than in the wildtype. These results suggested an involvement of $A B A$ in the volatile-induced root response, though not as hypothesized.

A

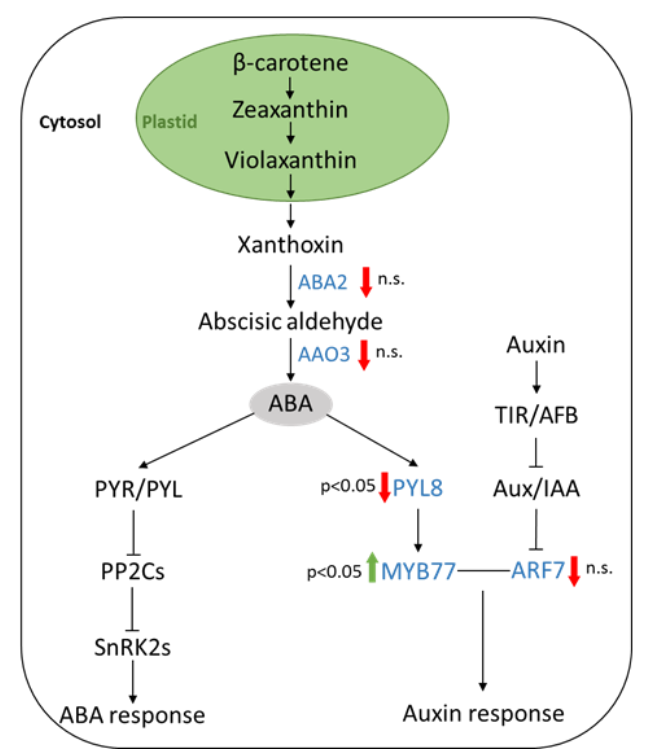

B

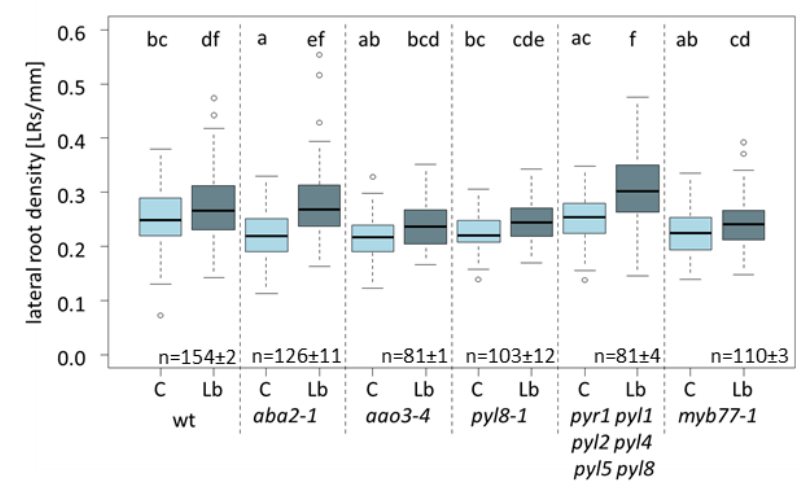

Figure 39 A) Scheme for ABA biosynthesis and signaling pathway in A. thaliana. ABA synthesis starts in plastids. Carotenoids are converted to zeaxanthin and zeaxanthin to violaxanthin. In a series of reactions, violaxanthin is converted to xanthoxin, which is transported in the cytosol. Xanthoxin is catalyzed to abscisic aldehyde by ABA2. AAO3 is responsible for the conversion of abscisic aldehyde to $A B A$. ABA binds to PYR/PYL receptors leading to the inhibition of PP2Cs and facilitating the SnRK2 activation in the common $A B A$ signaling pathways. An alternative signaling pathway during recovery after salt stress suggested that ABA activates PYL8 and MYB77. MYB77 bind to ARF7 leading to activation of the auxin response (Zhao et al., 2014). Proteins are shown in blue. Colored arrows indicate up- (green) or downregulation (red) of the gene in transcriptomic data of lateral root parts exposed to volatiles of L. bicolor. n.s. indicates 
that regulation was not significant. B) LR density of ABA-mutant-lines. Four-day-old seedlings were exposed to volatiles of $L$. bicolor for 7 days, according to the protocol in chapter 2.1.4. Six petri dishes per line and treatment with 15 seeds per dish were prepared. For the sextuple mutant, 14 petri dishes were prepared because many seeds did not germinate. Since not all seeds germinated the number of analyzed plants per line varied between treatments and lines as indicated by $\mathrm{n}$ in the figure. Data originated from 3 independent experiments. For statistical analysis, an ANOVA with number of experiments as random factor was performed. Different letters above the bars indicate significant differences at $p<0.001$, according to post-hoc Tukey's test. $n$, number of plants per treatment, C, control; Lb, L. bicolor

\subsubsection{No VALR (At4g31330) homolog in A. thaliana}

Among all treatments, the gene VALR (At4g31330, Protein of unknown function, domain of unknown function, DUF599) showed the most consistent response in roots exposed to volatiles of L. bicolor (Table S3, Table S4). Across all data, VALR was significantly downregulated. Therefore, we were interested to gain further knowledge on this gene. For this purpose, we searched for homolog proteins in A. thaliana. We found six other proteins of unknown function with the DUF599 domain by BLASTP alignment. We load the amino acid sequences of those proteins into Geneious Prime (v. 2020.2.2) and aligned them using Clustal Omega Alignment with the default settings. We defined a protein a "homolog" if the amino acid identity between VALR and the protein was $80 \%$ or higher. This was not the case for any of the six proteins with the DUF599 domain (Figure 40). Consequently, there was no VALR homolog protein in A. thaliana. Thus, $V A L R$ redundancy was unlikely and mutations of $V A L R$ were expected to affect the volatile-induced LR response. 


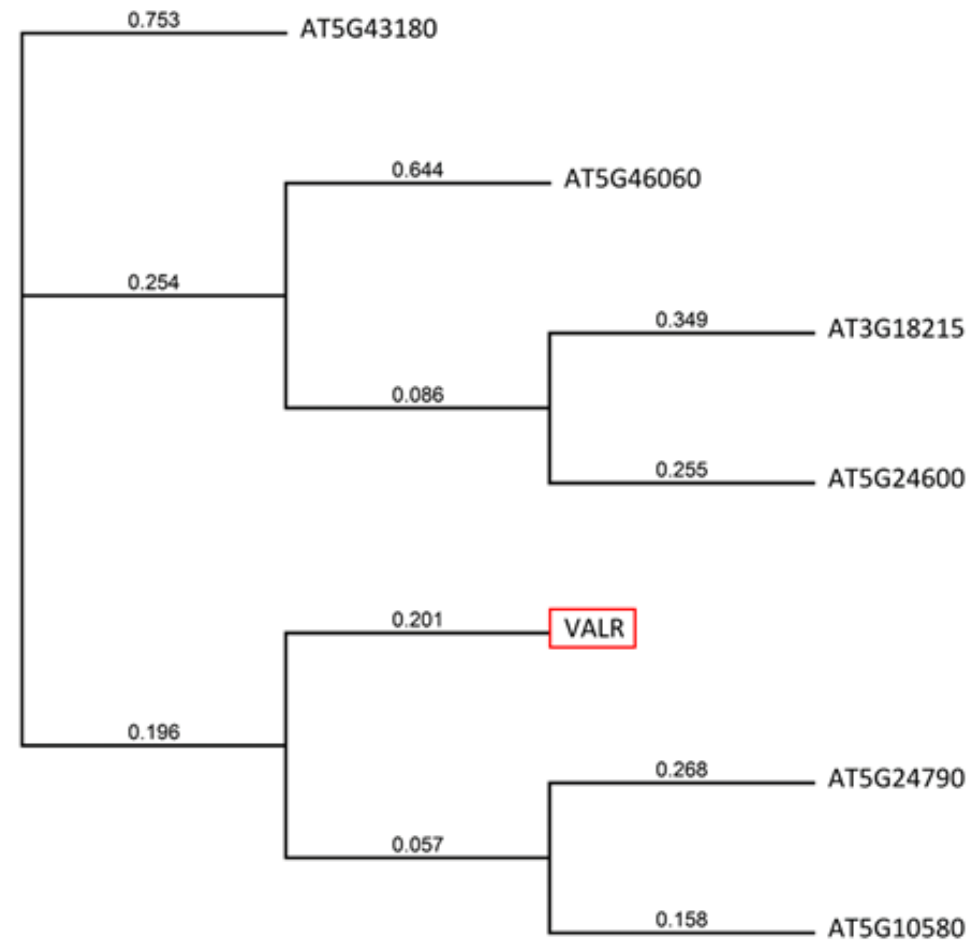

\section{Identities}

\begin{tabular}{|l|c|c|c|c|c|c|c|}
\hline & AT5G43180 & AT5G24790 & AT5G10580 & VALR & AT5G46060 & AT3G18215 & AT5G24600 \\
\hline AT5G43180 & & $30 \%$ & $31 \%$ & $31 \%$ & $20 \%$ & $24 \%$ & $27 \%$ \\
\hline AT5G24790 & $30 \%$ & & $63 \%$ & $56 \%$ & $23 \%$ & $29 \%$ & $32 \%$ \\
\hline AT5G10580 & $31 \%$ & $63 \%$ & & $67 \%$ & $27 \%$ & $32 \%$ & $35 \%$ \\
\hline VALR & $31 \%$ & $56 \%$ & $67 \%$ & & $29 \%$ & $33 \%$ & $36 \%$ \\
\hline AT5G46060 & $20 \%$ & $23 \%$ & $27 \%$ & $29 \%$ & & $35 \%$ & $34 \%$ \\
\hline AT3G18215 & $24 \%$ & $29 \%$ & $32 \%$ & $33 \%$ & $35 \%$ & & $51 \%$ \\
\hline AT5G24600 & $27 \%$ & $32 \%$ & $35 \%$ & $36 \%$ & $34 \%$ & $51 \%$ & \\
\hline
\end{tabular}

Figure 40. Phylogeny of VALR (At4g31330) in A. thaliana. Amino acid sequence of VALR was used in BLASTP (Phytozome 12 Blast Tool; https://phytozome.jgi.doe.gov/pz/portal.html\#!search?show=BLAST) and blasted against the $A$. thaliana TAIR10 genome. The amino acid sequences of those proteins were aligned in Geneious Prime (v. 2020.2.2) usind Clustal Omega Alignment. All proteins in the phylogenetic tree are protein of unknown function and contain a DUFF599 domain. Amino acid identities are shown in the table. 


\subsubsection{In silico analysis of VALR}

Not much is known about the VALR (At4g31330) gene or protein. VALR is a protein of 239 amino acids $(27.3 \mathrm{kDa})$. The $V A L R$ gene consists of 2 exons and 1 intron. The genomic DNA comprises $800 \mathrm{bp}$ from start to stop codon. We found that VALR is predicted to be a transmembrane protein because it contains four transmembrane domains according to the InterPro prediction website (https://www.ebi.ac.uk/interpro/). To find out, in which membrane VALR location was predicted, we used different localization prediction tools. SUBA (Subcellular Localization Database for Arabidopsis; https://suba.live/) predicted VALR to be in the membrane of the vacuole, endoplasmic reticulum, nucleus, mitochondrion or the Golgi apparatus. Furthermore, VALR localization in the plasma or the extracellular membrane was predicted by SUBA. According to LocTree3 Prediction (https://rostlab.org/services/loctree3/) VALR is located in the membrane of the endoplasmic reticulum (Score: 38, Expected Accurary $88 \%$ ).

To gain further knowledge on the protein we used the Arabidopsis eFP Browser (http://bar.utoronto.ca/efp/cgi-bin/efpWeb.cgi). We found that VALR is expressed in the pericycle of the primary root, which is the origin of lateral roots (Figure 41A) (Casimiro et al., 2003; Dolan et al., 1993; Dubrovsky and Rost, 2012). Moreover, according to the eFP browser, VALR is suppressed during lateral root initiation in wildtype (Col-0) plants. In the s/r-1/iaa14 mutant line, which is defective in LR formation, VALR is less suppressed than in wildtype roots (Figure 41B; eFP Browser). Furthermore, upon treatment with the auxin analog NAA (1-naphthaleneacetic acid), VALR was also suppressed (eFP Browser). However, when treated with naxillin, which induces LR formation with less transcriptional changes than NAA, no changes in VALR expression were observed (eFP Browser). VALR expression is also suppressed in the xylem pole pericycle during LR formation induction, the origin of LR primordia. Overall, these results point to a role of $V A L R$ in LR development. 
A

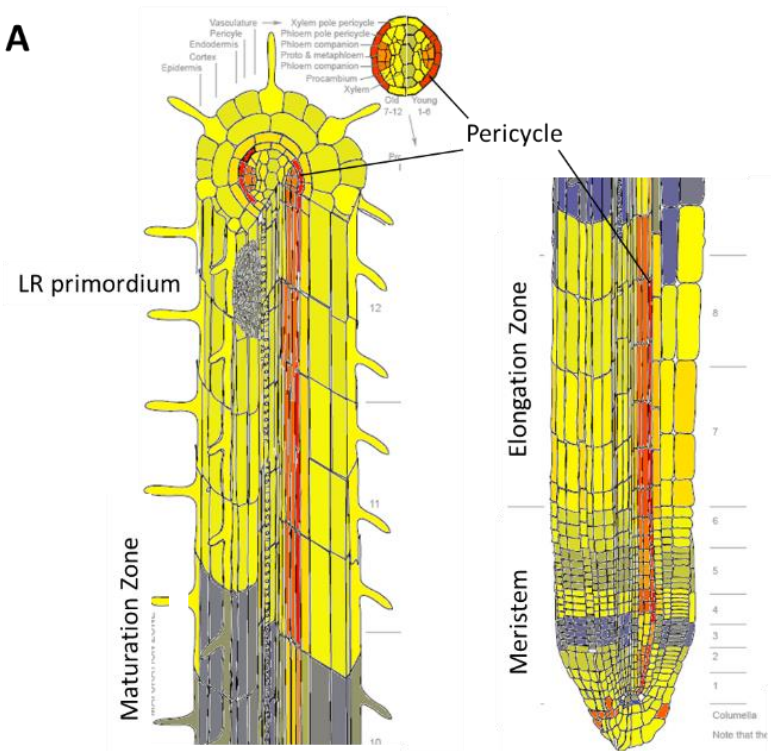

B

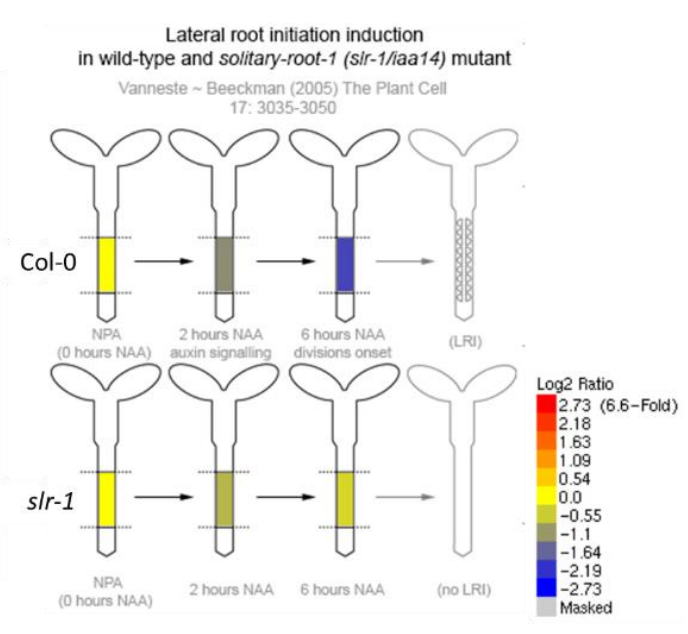

Figure 41. VALR expression in A. thaliana roots. A) expression in primary root parts $\mathrm{B}$ ) expression during LR formation. Figures were obtained from the eFB Browser (http://bar.utoronto.ca/efp/cgi-bin/efpWeb.cgi).

\subsubsection{Overexpressing VALR T-DNA insertion lines show less increase in LR density than the wildtype}

According to transcriptomic data and in silico analysis, VALR was a promising candidate involved in volatile-induced LR formation. To obtain functional information, we studied three T-DNA insertion lines of At4g31330 (VALR). The three T-DNA insertion lines available at NASC (Table 14) carried the T-DNA upstream of the gene (Figure 42A) in the promotor region. After genotyping (chapter 2.4), two homozygous lines remained: valr1 (GK-224D12) and valr2 (SAIL_692_C02). In the following experiment, the two homozygous sublines valr1-5 and valr1-10 were investigated together with the wildtype (Col-0). The sterilized seeds were co-cultivated with L. bicolor from the start of the experiment until the end after 11 days and then used for LR density determination. Since VALR was downregulated upon treatment with volatiles we expected higher LR density in valr T-DNA insertion lines compared to wildtype. In contrast to those expectations, valr1-5, valr1-10 and valr2 formed fewer lateral roots than the wildtype upon exposure to volatiles (Figure 42B). However, under control conditions, no difference between LR densities of wildtype and valr lines was found (Figure 42B). 
These findings are contrary to our hypothesis but fit to the expectation that overexpression lines have decreased LR densities compared to wildtype upon volatile exposure. Therefore, we tested VALR expression in the T-DNA insertion lines by qRT-PCR with cDNA from control roots. VALR expression was increased in T-DNA insertion lines (Figure 42C). Thus, the results from the overexpressing T-DNA insertion lines fit to our hypothesis for overexpression lines.

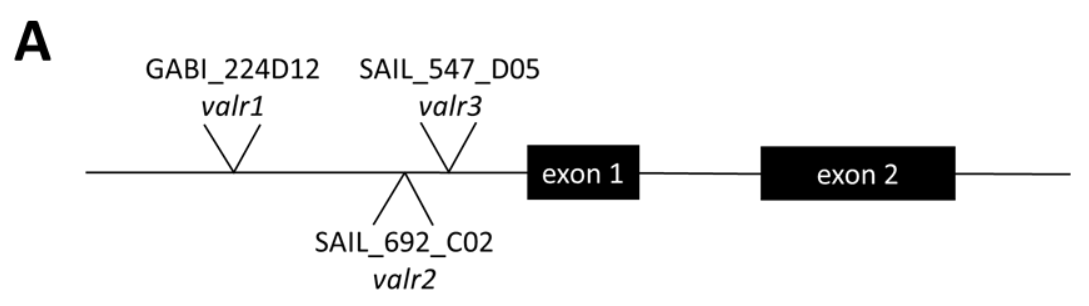

B

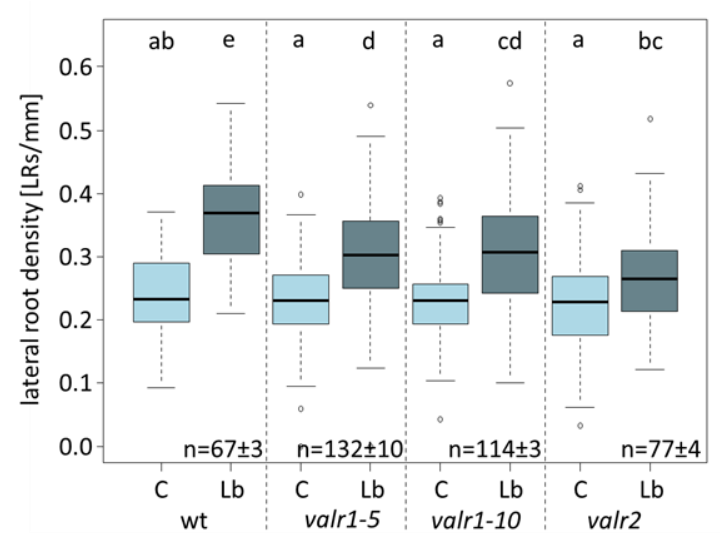

C

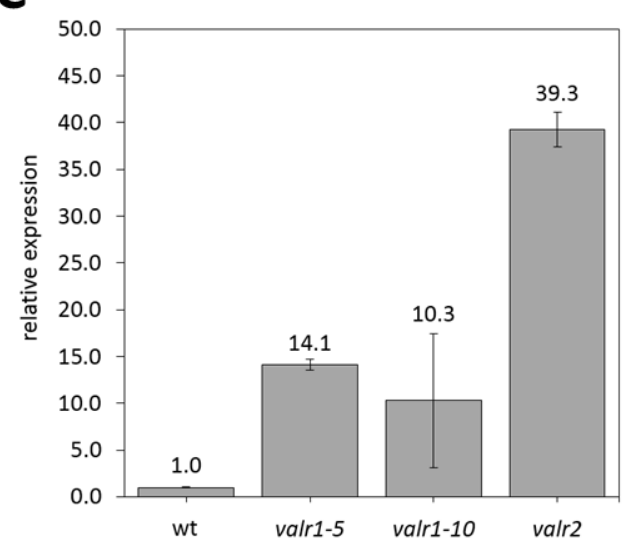

Figure 42. Valr T-DNA insertion lines. A) The T-DNA of the valr lines was localized in front of the VALR gene according to SIGnAL (http://signal.salk.edu/cgi-bin/tdnaexpress). The gene contains 2 exons and 1 intron. B) LR density of valr T-DNA insertion lines. Seeds were exposed to volatiles of L. bicolor for 11 days, according to the protocol in chapter 2.1.4. Six petri dishes per line and treatment with 15 seeds per dish were prepared. Since not all seeds germinated the number of analyzed plants per line varied between treatments and lines as indicated by $n$ in the figure. Data originated from one experiment. For statistical analysis, an ANOVA was performed. Different letters above the bars indicate significant differences at $\mathrm{p}<0.001$, according to post-hoc Tukey's test. $\mathrm{n}$, number of plants per treatment, C, control; Lb, L. bicolor. C) Relative normalized VALR expression in roots compared to wildtype. Roots from the experiment in $B$ ), which grew under control conditions, were pooled from 2 petri dishes. qRT-PCR was performed as described in chapter 2.10.9. Primer RT_AtDUF599rev_2 and RT_AtDUF599for_2 were used (Table 15). 


\subsubsection{Creating loss-of function VALR mutants using CRISPR/Cas9}

Since the VALR T-DNA insertion lines turned out to be overexpression lines, we mutated VALR in A. thaliana using CRISPR/Cas9 (modified from Wang et al., 2015 and Xing et al., 2014). To delete VALR (At4g31330) we choose two target sites, one at the beginning and one at the end of the gene (chapter 2.12.4). A scheme of the workflow for the CRISPR/Cas9-mediated generation of loss-of function mutant lines is presented in Figure 43. We transformed A. thaliana plants (F0) with the At4g31330-CRIPSR/Cas9 plasmid by Agrobacterium mediated transformation (chapter 2.12.3) by floral dip method (chapter 2.12.5). After maturation of the seeds, they were germinated on selection medium to identify positively transformed plants (F1) (chapter 2.12.6.2) Twelve days later, seedlings were transferred to soil to grow further. To detect VALR mutations a leaf from each plant was tested by PCR with the T7E1 (T7 endonuclease 1) assay (chapter 2.12.6.2). This enzyme tracks DNA mismatches and cleaves it at this site. Thereby, one can detect if non-homologous end joining DNA repair after cleavage through the Cas9 enzyme led to nucleotide insertions or deletions. If the size of the mutated PCR products was smaller compared to wildtype PCR products, the gene was deleted. In our case, all PCR products had the size of wildtype PCR product. Thus, the gene was not deleted in any line.

The resulting wildtype PCR products of VALR were annealed to mutant PCR products to be able to detect homozygous mutations. If T7E1 cleavage was detected, the tested mutant was either heterozygous or homozygous for the VALR mutation. To identify these mutations, we performed a second T7E1 assay on plants with cleaved products. This time, annealing to wildtype PCR-product was omitted. Here, cleavage indicated heterozygous mutations, while no cleavage indicated homozygous mutation, if the first assay showed a cleaved product. We found two potentially heterozygous lines (valr4 and valr6) and one potentially homozygous line (valr1) (Figure 44A). Sequencing confirmed the heterogeneity of valr4 and valr6. The sequencing stopped due to superimposed signals, which is a sign for heterogeneity. Valr1 turned out to be wildtype. However, this line was important later as a control. 


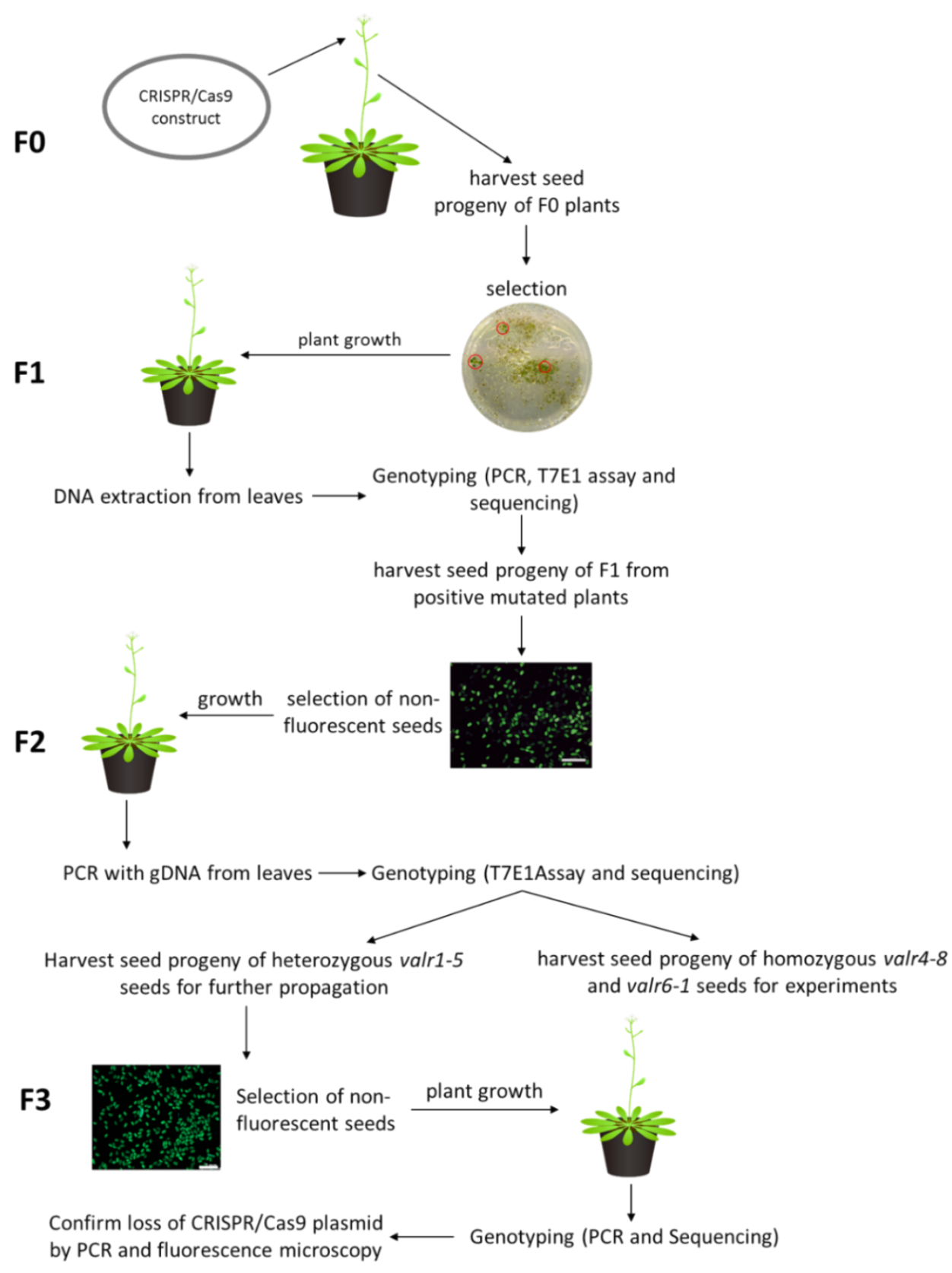

Figure 43. Scheme of CRISPR/Cas9-mediated generation of VALR loss-of function mutant lines. FO plants were transformed with the CRISPR/Cas9-containing plasmid by Agrobacterium mediated transformation. Seeds of the transformed plants were harvested and selected on selective media. The surviving plants (F1) were grown in soil. Their leaves were harvested and genotyped. The harvested seeds from F1 plants were investigated under the microscope und non-fluorescent seeds were chosen for growing the F2 plant generation. The genotyping of the leaves was repeated. Thereby valr4-8 and valr6-1 were identified as homozygous lines, whereas valr1-5 was heterozygous. Thus, seeds of valr1-5 were grown to the F3 generation, which were genotyped again. Afterwards, loss of CRISPR/Cas9 plasmid was confirmed using PCR and fluorescence microscopy of the progenies. 
A T7E1 Assay

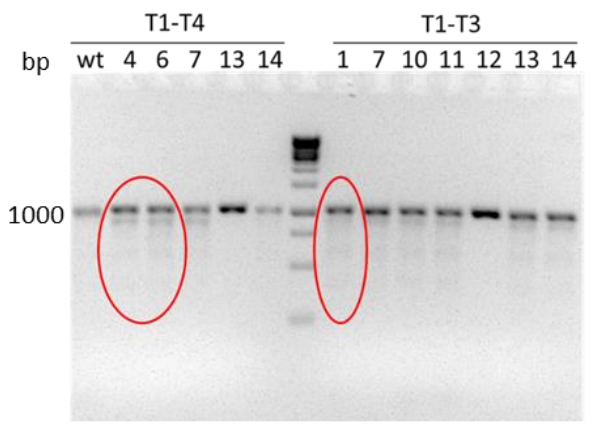

B PCR and sequencing of the products

\begin{tabular}{|c|c|c|}
\hline & target site 4 & \\
\hline VALR & CTAGAGAGAGGGTTTATTTTGAATACTGTGGGAATCGGTTGTTCTACGCGGCTTTGCCG & 2460 \\
\hline valr4_for & CTAGAGAGAGGGTTTATTTTGAATACTGTGGGGAATCGGTTGTTCTANNN---------- & 610 \\
\hline valr4_rev & 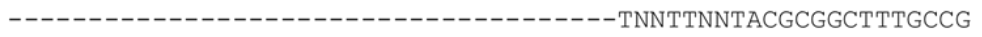 & 22 \\
\hline valre for & CTAGAGAGAGGGTTTATTTTGAATACTGTGGGGAATCGGTTGTTCTNNNN----------- & 613 \\
\hline valr6_rev & 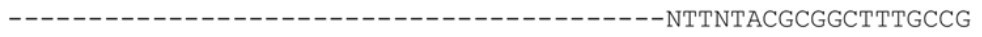 & 19 \\
\hline
\end{tabular}

Figure 44. Identification of positively mutated valr lines (F1). A) Unmixed T7E1 Assay of plants transformed either with the CRISPR/Cas9 target sites 1 and 4 (T1-T4) or with the target sites 1 and 3 (T1-T3). Lines used in this assay were validated in a mixed T7E1 assay before (chapter 2.12.6.2). Line 4 and 6 were grown to the next generation. Line 1 was grown to the next generation as segregated wildtype. wt, wildtype B) Sequencing results of valr4 and valr6. Sequences stopped in target site 4 due to superimposed signals.

Next, we observed the seed progeny of F1 plants under the microscope and selected non-fluorescent seeds for further analyses. The CRISPR/Cas9 plasmid contained GFP, controlled by an egg-cell specific promotor (Figure 11). Thereby, we were able to distinguish between seeds harboring the plasmid and those, which did not inherit it. Due to the chance of unspecific Cas9 cleavage, we avoided further work with CRISPR/Cas9 containing seeds. We selected non-fluorescent seeds of valr4, valr6 and other promising lines and transferred them to soil. We also chose valr1, the segregated wildtype for control experiments. After rosette development of the F2 plants, we harvested leaves, performed the T7E1 assay and sequenced the PCR product generated for the T7E1 assay (chapter 2.12.6.2). During mixed T7E1 assays, valr4-8 and valr6-1 showed cleaved products (Figure 45A). Besides, valr1-5 was mutated as well, although the parent F1 plant was not mutated (Figure 45A). This indicated that F2 plants inherited the CRISPR/Cas9 plasmid from the valr1 F1 generation.

Sequencing revealed a homozygous T-insertion three nucleotides upstream of the PAM (protospacer adjacent motif) site of CRIPSR/Cas9 target site 4 in valr4-8 and valr6-1. 
The insertion led to a frameshift, which resulted in the formation of premature stop codons and the change of the code for amino acids (Figure 45B). However, the T-insertion was close to the end of the gene. Therefore, it is uncertain if the function of the protein was lost.

Sequencing of valr1-5 revealed a heterozygote mutant line, which lost a major fraction of the gene ( $560 \mathrm{bp}$ ) between CRISPR/Cas9 target sites 1 and 3 (Figure 45B). We grew this line to the next generation. After seed maturation, we tried to choose seeds without fluorescence but could not find any (Figure 46A). However, we still germinated the seeds. After 20 days of growth, leaves of the developing plants (F3) were genotyped with PCR. If the gene was deleted, the expected PCR product had a size of $790 \mathrm{bp}$. We found 5 plants in the valr1-5 progeny which lost the gene between target site 1 and 3 (Figure 46B). In addition, upstream of the Cas9 cleavage site, 2 nucleotides were inserted and 4 were exchanged. The resulting truncated VALR protein had a size of 79 amino acids and included 3 stop codons. Hence, we assume that the function of the gene was massively disturbed (Figure 46B). 
A T7E1 Assay

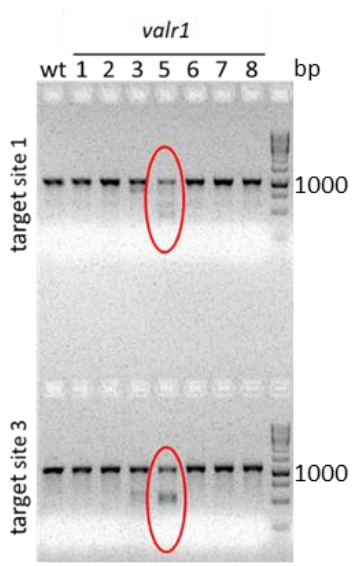

B Sequencing PCR products

valr4-8 and valr6-1

\begin{tabular}{|c|c|c|c|}
\hline & & target site 4 & \\
\hline & VALR & CTAGAGAGAGGTTTATTTTGAATACTGTGGGGAATCGGTTGTTCT-ACGCGGCTTTGCC & 2459 \\
\hline & valr4-8_for & CTAGAGAGAGGTTTATTTTGAATACTGTGGGGAATCGGTTGTTCTTACGCGGCTTTGCC & 612 \\
\hline 5 & valr4-8_rev & CTAGAGAGAGGGTTTATTTTGAATACTGTGGGGAATCGGTTGTTCTTACGCGGCTTTGCC & 648 \\
\hline & valr6-1_rev & CTAGAGAGAGGTTTATTTTGAATACTGTGGGGAATCGGTTGTTCTMACGCGGCTTTGCC & 651 \\
\hline & & target site 4 & \\
\hline & Consensus & GNRLFXXXXXXXXXXXJWXXXXXVXVXXXXXXXXXXXXXXXXXFXXXXXXXXKXXXKXXFXXXV & 243 \\
\hline$\overline{0}$ & VALR & GNRLFYAALPL---MLWIFGPVLVFLCSVVMVPLLYNLDFFFFGKERRKLDQKSSFGSV- & 239 \\
\hline & valr4-8 & GNRLFLRGFAVDALDIWTGACVFVLGRD--GS-STLQPRFLLLR* RKKKT*SKV*FWICV & 240 \\
\hline & valr6-1 & 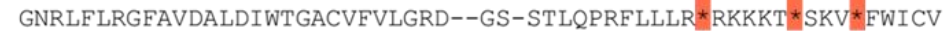 & 240 \\
\hline
\end{tabular}

valr1-5

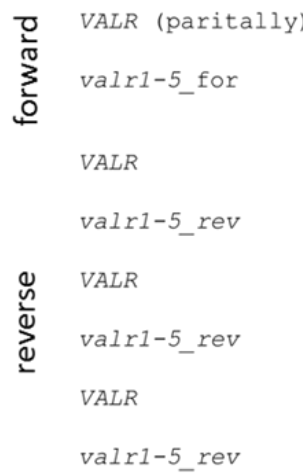

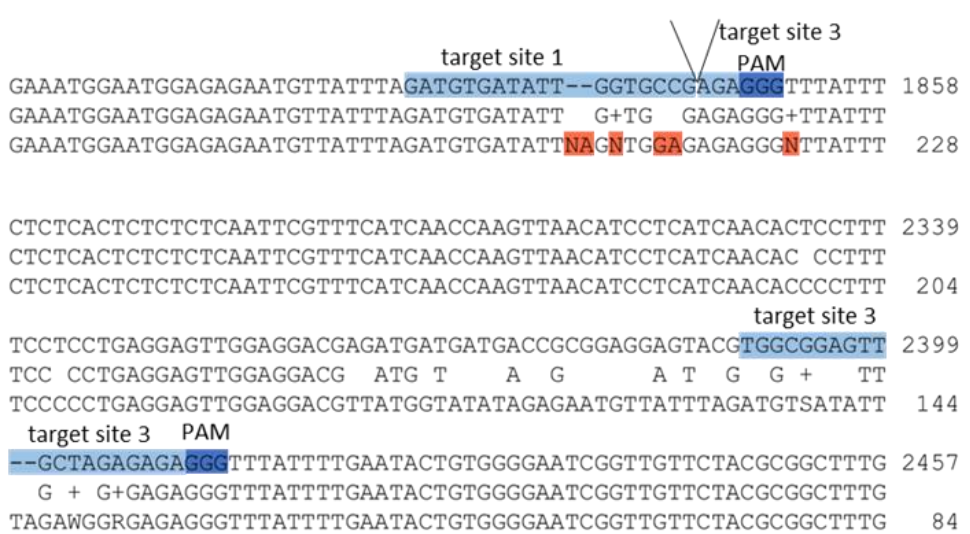

Figure 45. Genotyping of valr4-8 and valr6-1 lines (F2 generation). A) Mixed T7E1 Assay with target site specific PCR products. Lines valr4-8 and valr6-1 were sequenced. In addition, valr1-5 was mutated and sequenced. wt, wildtype. B) Sequencing results of PCR products of valr4-8, valr6-1, and valr1-5. The part of interest of the VALR gene is shown. Valr4-8 and valr6-1 had a T-insertion at the CRSIPR/Cas9 cleavage site, leading to a frameshift and three premature stop codons. Valr1-5 was heterozygote for a gene deletion between target site 1 and 3 (indicated by a $V$ ) as seen for the forward sequence. Sequencing from the reversed site revealed no distinct sequence as well. 
A

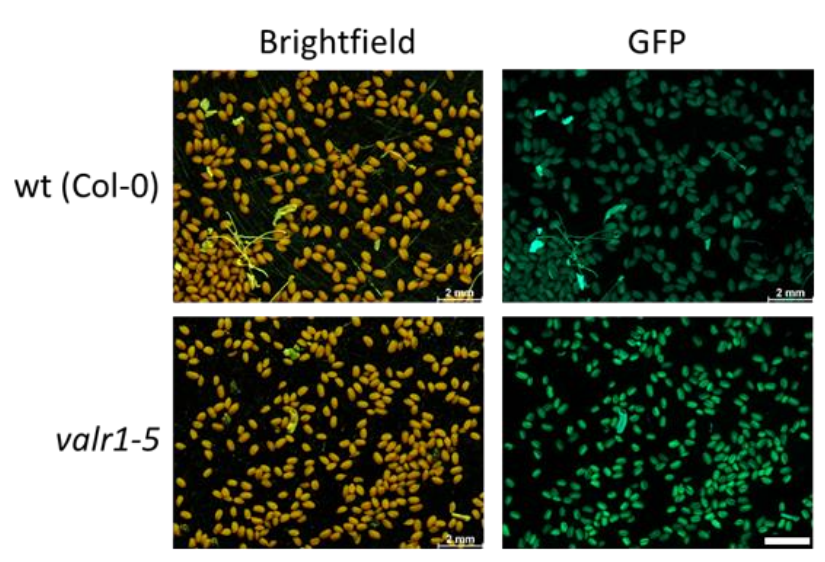

B
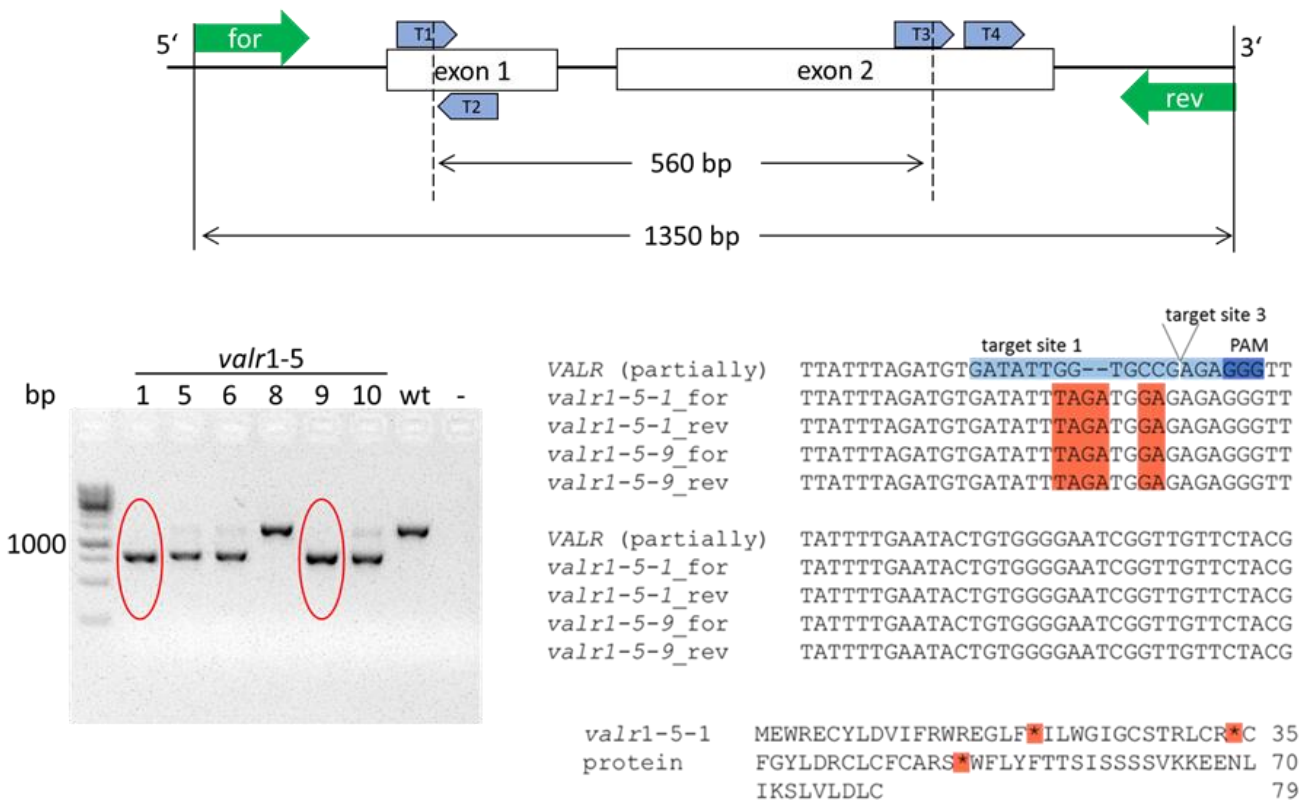

Figure 46. A) Fluorescence signal in valr1-5 seeds. Selection of non-fluorescent seeds was not possible since nearly all seeds showed a signal. Fifteen seeds were selected for growing. Scale bar $=2 \mathrm{~mm}$. B) Genotyping of valr1-5 progeny. Primer were chosen outside of the gene to detect gene deletions. PCR and sequencing showed that some progenies of valr1-5 lost most of the gene indeed. Line valr1-5-1 and valr1-5-9 were sequenced. The $V$ indicates the missing gene part in the VALR gene. The deletion and insertion lead to a truncated protein, which contains three stop codons. wt, wildtype. 


\subsubsection{Inheritance of CRSIPR/Cas9 T-DNA in valr-mutants}

As described in 3.6.5, we tried to remove the CRISPR/Cas9 T-DNA by outcrossing to avoid unspecific cleavage. To test, whether plants inherited the CRISPR/Cas9 T-DNA, we conducted a PCR with GFP-specific primers (chapter 2.10.6, Table 15) to detect the T-DNA. If the PCR revealed a product, the GFP was amplified, indicating that the CRISPR/Cas9 T-DNA was still present. From the lines valr4-8 and valr6-1 no GFP product was amplified (Figure 47A). In contrast, in the progeny of valr1-5 (valr1-5-1 and valr1-5-9) GFP was amplified, indicating inheritance of the CRISPR/Cas9 T-DNA (Figure-47A). Furthermore, we investigated the seed progeny of the valr-plants with a fluorescence stereomicroscope. While the seed progeny of valr4-8 and valr6-1 did not show a fluorescence signal, seeds of valr1-5-1 and valr1-5-9 did so (Figure 47B) supporting the PCR results. Nevertheless, since valr1-5-1 and valr1-5-9 were the only line which lost most of the gene, we decided to include those seeds in further experiments.

A

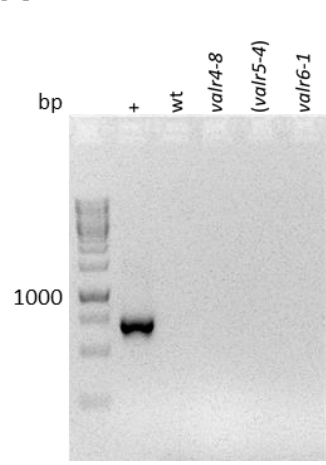

B

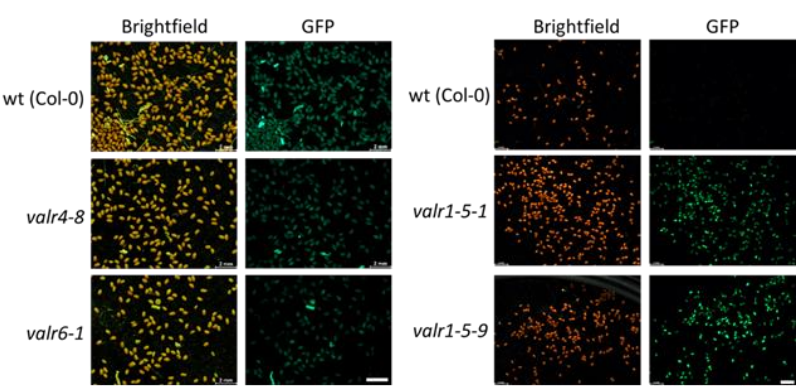

Figure 47. GFP in valr mutant lines. A) PCRs with GFP specific primers were performed with genomic DNA from leaves (chapter 2.10.6). If a product was amplified, the plant inherited the CRISPR/Cas9 construct. $+=$ CRISPR/Cas9 vector, wt, wildtype, B) Seed fluorescence of the progeny of the stated lines (chapter 2.12.6.2). The lines valr1-5-1 and valr1-5-9 inherited the CRISPR/Cas9 plasmid. Scale bar $=2 \mathrm{~mm}$. 


\subsubsection{Volatile-induced LR formation in valr-mutants varies}

To test whether volatiles of L. bicolor affected LR formation in valr4-8, valr6-1, valr1-5-1 and valr1-5-9, we grew seedlings for 4 days before adding 14-day-old fungal mycelium in bi-compartmented petri dishes. After 7 days of exposure to volatiles, the plants were used for determination of the LR density. We hypothesized that L. bicolor volatileinduced LR formation is increased in VALR suppressed mutants compared to wildtype. During visual analysis, we noticed shorter primary roots in individual plants (Figure 48A). In addition, valr6-1 seems to form fewer lateral roots than wildtype. Analysis of the LR density showed indeed significantly 1.12-fold lower LR density in valr6-1 seedlings compared to wildtype under control conditions. The LR density of valr6-1 plants exposed to volatiles was also significant lower (1.07-fold) compared to wildtype (Figure 48B). Thus, the difference in LR density between treated and untreated samples of valr1-6 is the same as in wildtype.

The same was true for valr1-5-9. Under control conditions, LR density was 1.24-fold lower compared to wildtype. Under treatment with volatiles, the LR density was 1.18-fold lower compared to wildtype Figure 48B). The difference in LR density between treated and untreated samples of valr1-5-9 is the same as in wildtype. Taken together, valr6-1 and valr1-5-9 responded to fungal volatiles like wildtype plants but might be impaired in general LR formation due to the lower basal LR density.

Valr4-8 did not show any difference to wildtype, neither under control conditions, nor upon volatile exposure (Figure 48B). We expected that valr4-8 and valr6-1 show the same LR density and response to volatiles, because they have the same mutation based in the sequence (Figure 45B). However, valr6-1 showed a lower LR density in treated and untreated roots compared to valr4-8 (Figure 48B).

Valr1-5-1 showed similar LR density compared to wildtype under both conditions. However, the difference between untreated and treated plants of this line is not significant ( $p=0.095$ ) (Figure 48B). We expected, that valr1-5-1 and valr1-5-9 show the same behavior, because both lines lost the majority of the gene (Figure 46B).

Valr4-8 did not form less LRs compared to wildtype, neither under control conditions nor upon exposure to volatiles. Also, valr1-5-1 should behave like valr1-5-9 but it did not. Under both treatments, valr1-5-1 showed similar LR density compared to wildtype. 
After exposure to volatiles, we harvested roots grown under control conditions to test VALR expression in the valr4-8, valr6-1 and valr1-5-1 and valr1-5-9 line. The expression of VALR was reduced in each valr line. However, one must remember that valr4-8 and valr6-1 were mutated by a nucleotide insertion, which most likely does not affect the translation process to RNA, but probably the protein function. Therefore, the primer RT_AtDUF599for_2 and RT_AtDUF599rev_2 (Table 15) used for qRT-PCR can still bind to the synthesized CDNA. In contrast to the VALR expression of valr4-8 and valr6-1, we expected a relative expression to wildtype between 0 and 0.1 for valr1-5 1 and valr1-5-9, because most of the gene was missing and the primer were not able to bind to the cDNA. In roots of valr1-5-1 the relative expression of VALR to wildtype of VALR was low (0.08). The expression level in valr1-5-9 is 0.36 , which was not as close to 0 as we expected. Reason for this are unknown.

In summary, VALR expression was reduced in the mutant lines valr4-8, valr6-1, valr1-5-1, and valr1-5-9, but LR density was not enhanced, as expected on the basis of the previous results. Upon treatment with volatiles, valr lines showed rather a lower LR density compared to wildtype, indicating a role in volatile-independent LR formation. However, the lower LR density was not consistent within the valr4-8, valr6-1, valr1-5-1, and valr1-5-9 lines. 
A

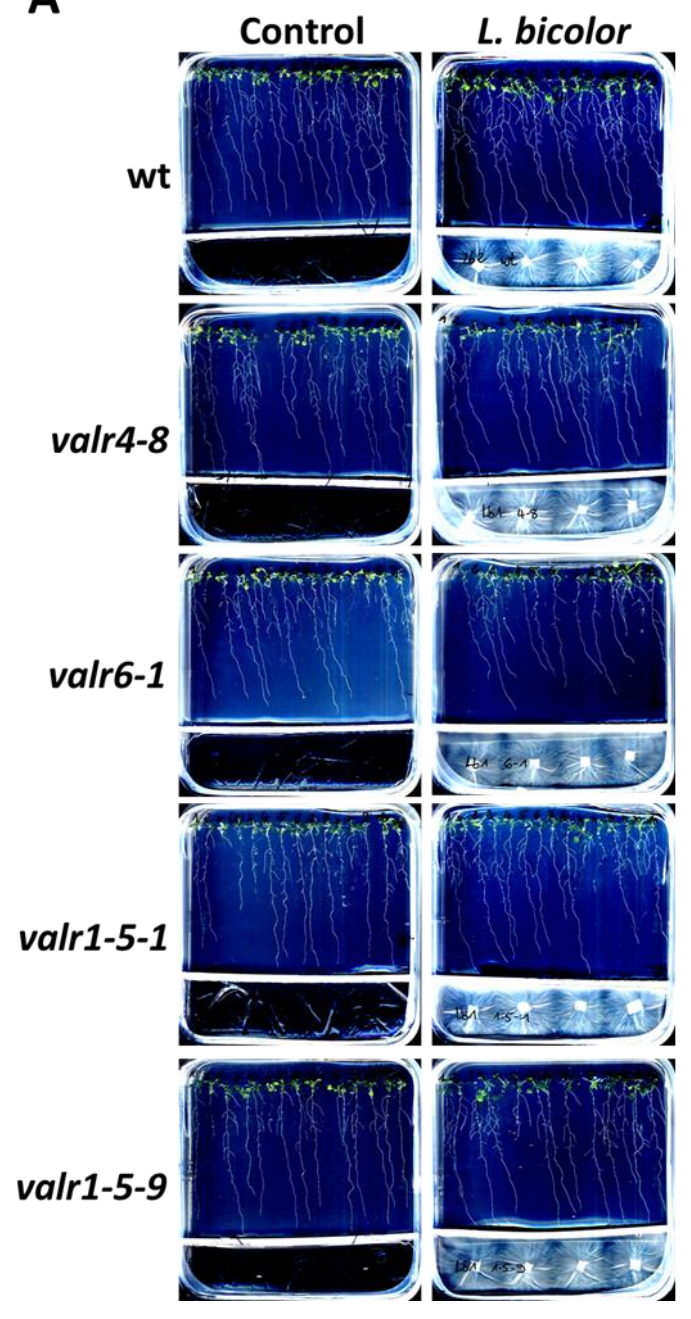

B

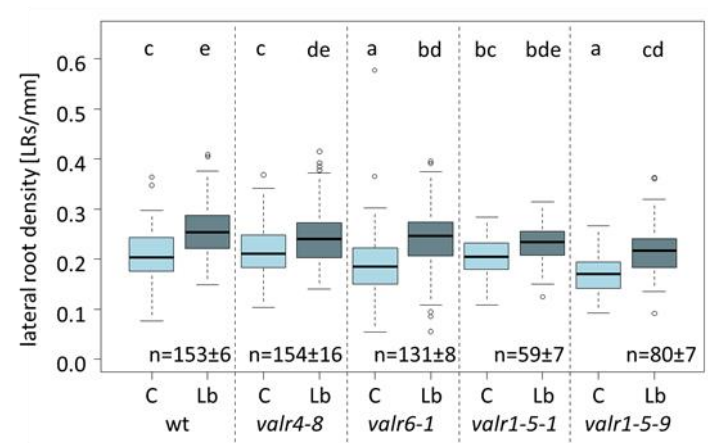

C

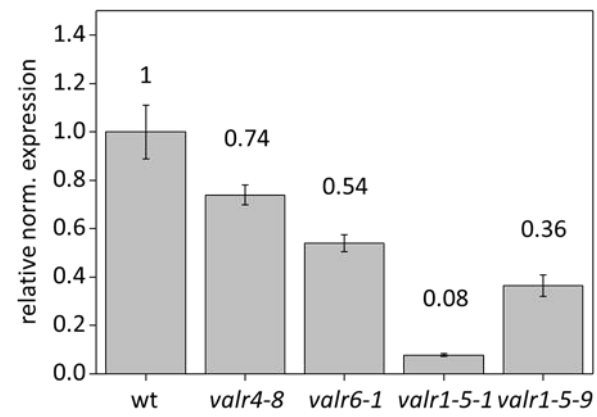

Figure 48. LR density of valr loss-of function lines. A) Typical examples of seedlings of valr lines, of which the LR density was determined. For analysis, 8 petri dishes with 15 seeds per line and condition were prepared. B) LR density of valr lines. Four-day-old seedlings were exposed to volatiles of L. bicolor for 7 days, according to the protocol in chapter 2.1.4. Since not all seeds germinated the number of analyzed plants per line varied between treatments and lines as indicated by $\mathrm{n}$ in the figure. Data originated from 2 independent experiments. For statistical analysis, an ANOVA with number of experiments as random factor was performed. Different letters above the bars indicate significant differences at $\mathrm{p}<0.001$, according to post-hoc Tukey's test. $\mathrm{n}$, number of plants per treatment, C, control; Lb, L. bicolor. C) Relative normalized VALR expression in roots of valr lines. Roots grown under control conditions from 2 petri dishes from one experiment were pooled for RNA extraction. qRT-PCR was performed as described in chapter 2.10.9. 


\subsubsection{Creating overexpression lines of VALR}

Overexpression lines of VALR (At4g31330) were created using the Gateway system (chapter 2.12.3). We cloned two overexpression constructs. In both constructs, the gene was controlled by the constitutive $35 \mathrm{~S}$ promotor. In one of the constructs, the promotor controlled the expression of eGFP in addition to VALR. Figure 49 shows a scheme to produce the overexpressing lines. A. thaliana plants (F0) were transformed either with the p35S::eGFP-At4f31330-HA expression clone or the p35S::At4g31330-HA expression clone. Before transformation, we confirmed the coding region of VALR in the backbone vector by sequencing with a vector specific primer (pK7WG-primer pair, Table 15). The coding sequence of VALR was correctly inserted in both backbone vectors (Figure 50). After seed maturation, overexpressing progenies were identified on selective media. We used leaves of F1 plants to test whether the plants contained the T-DNA with the p35S::eGFP-At4g31330-HA expression clone or the p35S::At4g31330-HA expression clone. For this purpose, we chose a forward primer (AtDUF599for_ex1) that binds to the VALR sequence and a reverse primer (pK7WG_rev) that binds to the plasmid sequence (Figure 51A; Table 15). Thereby, we ensured that products were only obtained if the VALR gene was inserted in the backbone vector. PCR products of the expected size of $913 \mathrm{bp}$ were found for both expression clones. We harvested the seeds of the positive mutated lines, among which were VALR-OEX1, -OEX2,-OEX6 and-OEX14, to grow the next generation.

We selected the progeny of F1 plants on selective media to exclude wildtype plants (chapter 2.12.6.1). F2 plants were grown and seeds matured. We harvested the seed progeny of F2 plants and identified homozygous plants by determining the growth rate of seeds of 6 plants per independent line on selective media (chapter 2.12.6.1). Nineteen plants of the 5 tested lines were homozygous and 11 were heterozygote (Table 19). We chose the GFP-expressing homozygote lines VALR-OEX6-4 and VALR-OEX14-3, and no GFP expressing lines VALR-OEX1-15 and VALR-OEX2-5 for further experiments. 
F0

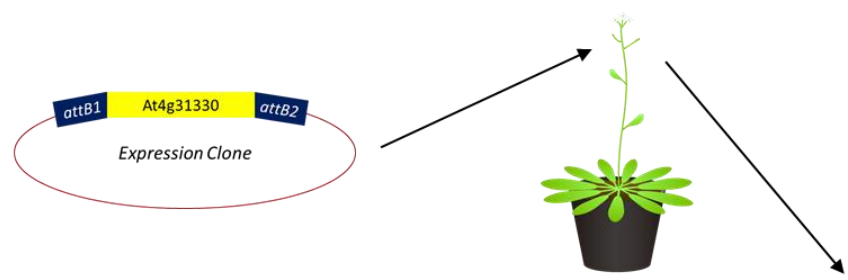

Harvest seed progeny of FO plants

F1

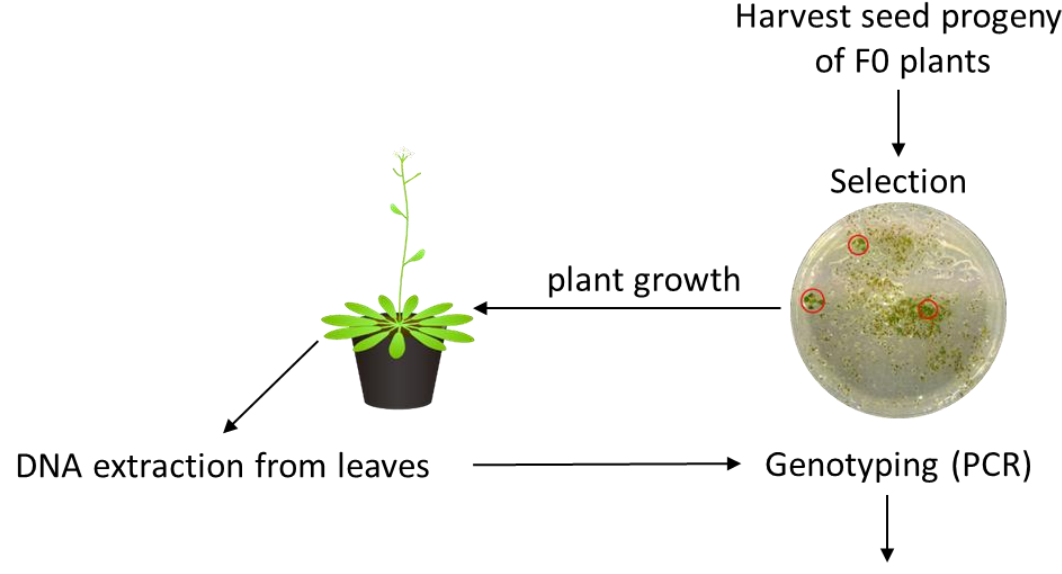

Harvest seed progeny of F1 from positive mutated plants

F2

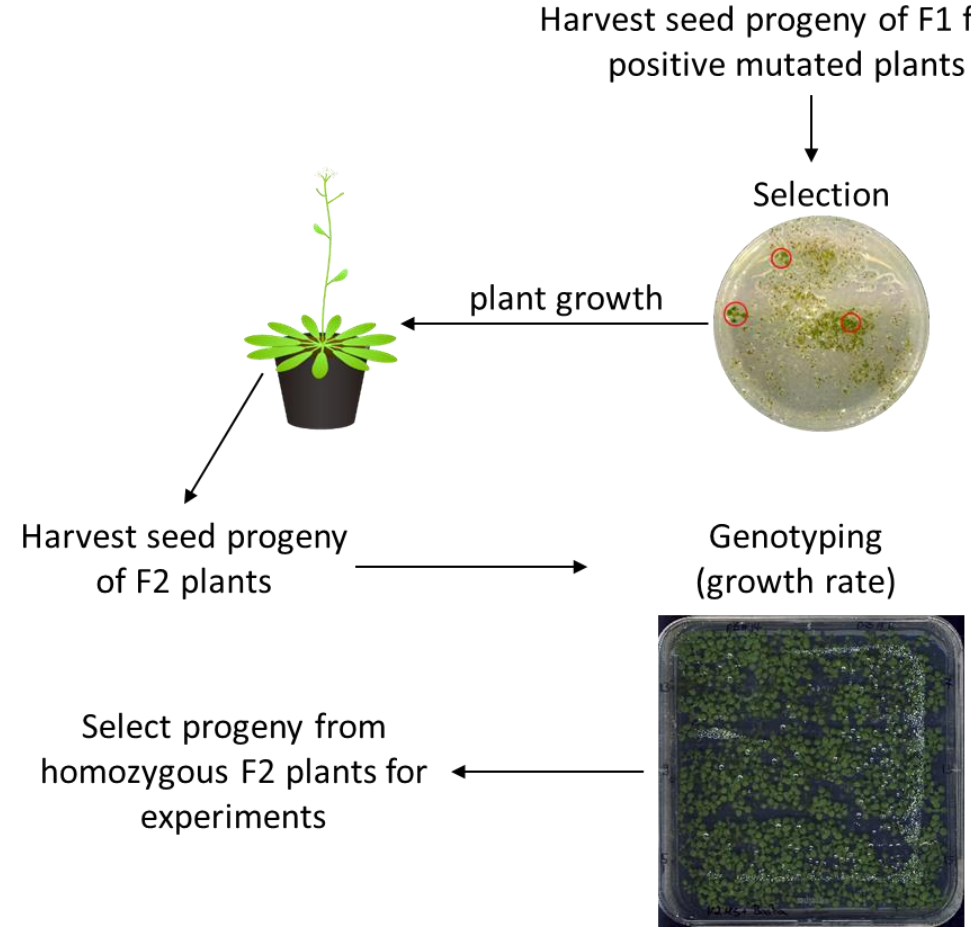

Figure 49. Scheme of creating VALR overexpression lines. F0 plants were transformed with the expression clone from the gateway cloning system. The progeny of the F0 plants was selected on selective medium. Only transformed seedlings remained. They were grown to F1 plants. These were genotyped to test for the T-DNA. The progeny of positive transformed F1 plants was selected again to exclude wildtype plants. Selected F2 plants were grown. Seeds of its progeny were used in a growth rate assay on selective medium to find homozygous F2 plants. 
A

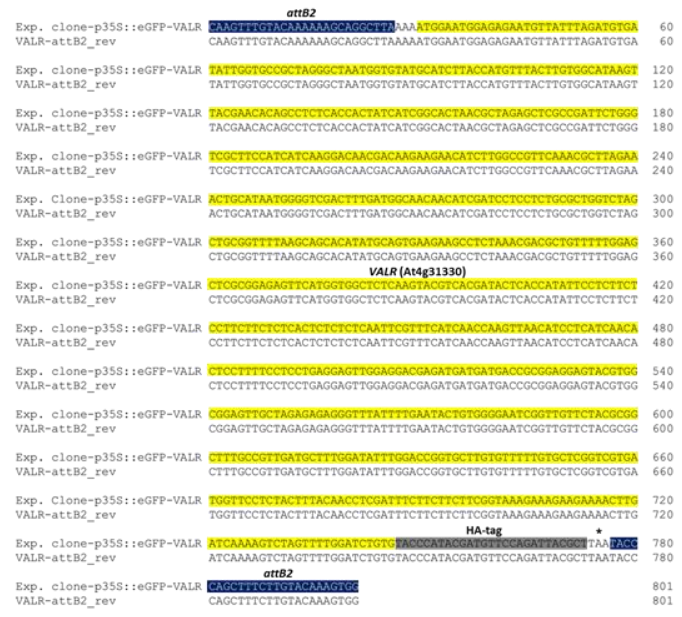

B

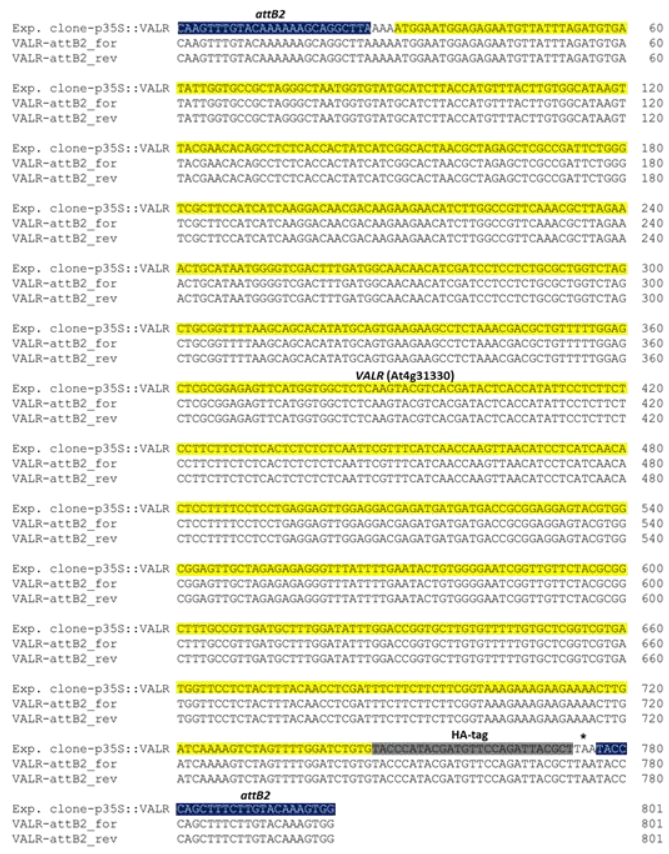

Figure 50. VALR coding sequences with attB2-sites, HA-tag and Stop-codon $\left(^{*}\right)$ in the eGFP containing expression clone (A) and the one without eGFP (B). A) Only one direction of the sequence is shown, because a plasmid specific primer was used, and it bind in front of the eGFP. Therefore, the eGFP and only parts of the gene were sequenced. B) Plasmid specific primer bind in front and after the VALR coding sequence, so the whole gene was sequenced in both directions.

A

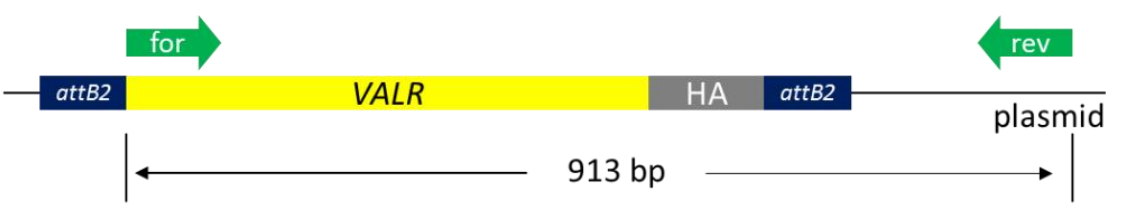

B

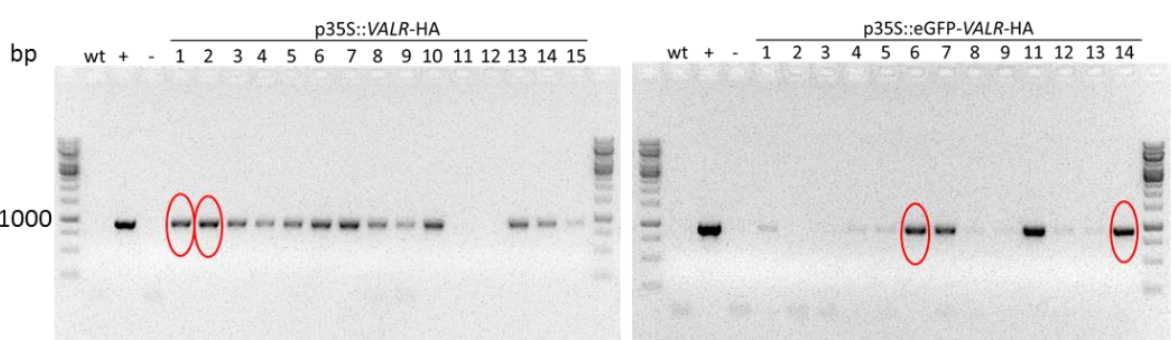

Figure 51. At4g31330-OEX. T-DNA insertion in F1 plants. A) Primer design for PCR. The forward (for) primer binds to the DNA of the gene, the revers (rev) Primer binds to the plasmid. The expected product is $913 \mathrm{bp}$ in size. B) Resulting PCR products. For both clones, positive transformed plants were identified. Red ellipses indicate the lines which we used for further work. + , expression clone; wt, wildtype 
Table 19. Growth rates of VALR overexpression lines on AM medium without antibiotics (AM) or with antibiotics. Six plants per line and 84 seeds per plant were tested in one petri dish. Lines with a growth rate of $90 \%$ to $100 \%$ on selective medium were considered as homozygote, lines with a growth rate of $89 \%$ and smaller were considered as heterozygote. Lines marked in yellow were used for the following experiments.

\begin{tabular}{|c|c|c|c|c|}
\hline Line & Mutation & AM & AM+Antibiotic & Genotype \\
\hline OEX14-1 & p35s::eGFP-VALR-HA & $97 \%$ & $94 \%$ & homozygote \\
\hline OEX14-13 & p35s::eGFP-VALR-HA & $99 \%$ & $95 \%$ & homozygote \\
\hline OEX14-3 & p35s::eGFP-VALR-HA & $94 \%$ & $100 \%$ & homozygote \\
\hline OEX14-5 & p35S::eGFP-VALR-HA & $100 \%$ & $96 \%$ & homozygote \\
\hline OEX6-4 & p35s::eGFP-VALR-HA & $96 \%$ & $100 \%$ & homozygote \\
\hline OEX6-7 & p35s::eGFP-VALR-HA & $97 \%$ & $94 \%$ & homozygote \\
\hline OEX1-1 & $p 35 s:: V A L R-H A$ & $100 \%$ & $91 \%$ & homozygote \\
\hline OEX1-14 & p35S::VALR-HA & $100 \%$ & $99 \%$ & homozygote \\
\hline OEX1-15 & p35S::VALR-HA & $100 \%$ & $100 \%$ & homozygote \\
\hline OEX1-6 & p35S::VALR-HA & $97 \%$ & $99 \%$ & homozygote \\
\hline OEX1-9 & p35S::VALR-HA & $100 \%$ & $96 \%$ & homozygote \\
\hline OEX2-1 & p35S::VALR-HA & $100 \%$ & $100 \%$ & homozygote \\
\hline OEX2-10 & $p 35 S:: V A L R-H A$ & $100 \%$ & $100 \%$ & homozygote \\
\hline OEX2-12 & p35S::VALR-HA & $100 \%$ & $97 \%$ & homozygote \\
\hline OEX2-13 & p35S::VALR-HA & $98 \%$ & $100 \%$ & homozygote \\
\hline OEX2-5 & p35S::VALR-HA & $100 \%$ & $100 \%$ & homozygote \\
\hline OEX2-7 & p35S::VALR-HA & $100 \%$ & $99 \%$ & homozygote \\
\hline OEX7-13 & p35S::VALR-HA & $100 \%$ & $98 \%$ & homozygote \\
\hline OEX7-5 & p35S::VALR-HA & $100 \%$ & $100 \%$ & homozygote \\
\hline OEX7-1 & p35S::VALR-HA & $100 \%$ & $85 \%$ & heterozygote \\
\hline OEX6-15 & p35s::eGFP-VALR-HA & $100 \%$ & $84 \%$ & heterozygote \\
\hline OEX6-13 & p35s::eGFP-VALR-HA & $99 \%$ & $82 \%$ & heterozygote \\
\hline OEX6-1 & p35s::eGFP-VALR-HA & $99 \%$ & $82 \%$ & heterozygote \\
\hline OEX1-7 & p35S::VALR-HA & $99 \%$ & $80 \%$ & heterozygote \\
\hline OEX14-9 & p35S::eGFP-VALR-HA & $99 \%$ & $79 \%$ & heterozygote \\
\hline OEX6-6 & p35S::eGFP-VALR-HA & $99 \%$ & $75 \%$ & heterozygote \\
\hline OEX14-2 & p35S::eGFP-VALR-HA & $92 \%$ & $73 \%$ & heterozygote \\
\hline OEX7-4 & p35S::VALR-HA & $96 \%$ & $62 \%$ & heterozygote \\
\hline OEX7-12 & p35S::VALR-HA & $100 \%$ & $27 \%$ & heterozygote \\
\hline OEX7-10 & p35S::VALR-HA & $100 \%$ & $11 \%$ & heterozygote \\
\hline
\end{tabular}




\subsubsection{VALR-overexpression lines did not respond to volatiles}

We used the progeny of four homozygote F2 lines for testing their response to volatiles of L. bicolor. In line with our previous results (chapters 3.6.4, 2.13). We hypothesized that overexpression of VALR leads to lower LR density in roots exposed to volatiles compared to wildtype. A higher number of seeds of OEX6-4 did not germinate compared to wildtype. Furthermore, primary roots of OEX6-4 seemed to be shorter compared to wildtype (Figure 52A). We determined the LR densities. All VALR-OEX lines behaved like wildtype. They showed the same increase in LR density upon treatment with volatiles of L. bicolor (Figure 52B), indicating no involvement in volatile-induced LR formation or in LR development. The VALR-OEX lines OEX2-5, OEX6-4 and OEX14-3 showed slightly shorter primary roots compared to wildtype (Figure 52C) but this decrease was only significant for OEX6-4 roots exposed to volatiles. In addition to the LR density and the primary root length, we investigated the VALR expression in roots of the OEX lines. For this purpose, we pooled roots of plants grown under control conditions and used them for each line separately for qRT-PCR. The tested VALR-OEX lines showed increased expression of VALR in the roots compared to wildtype (Figure 52D). We observed the highest increase of 959-fold in OEX6-4. OEX1-5 showed an increase of 156-fold, OEX14-3 of 50-fold and OEX2-5 of 25-fold. 


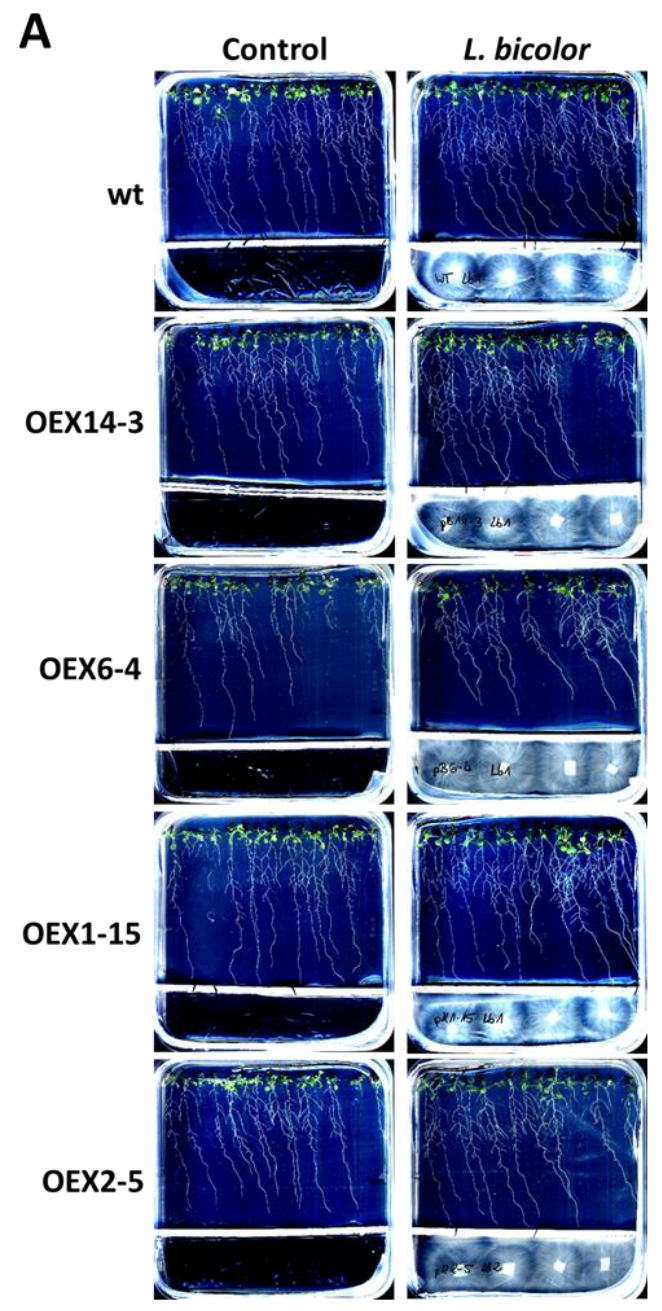

B

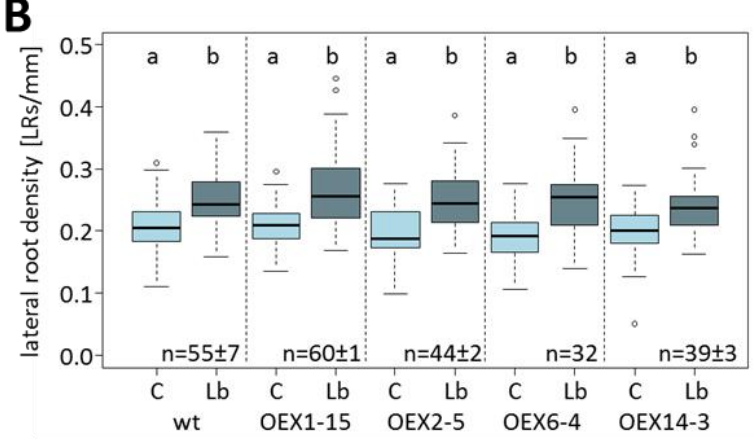

C
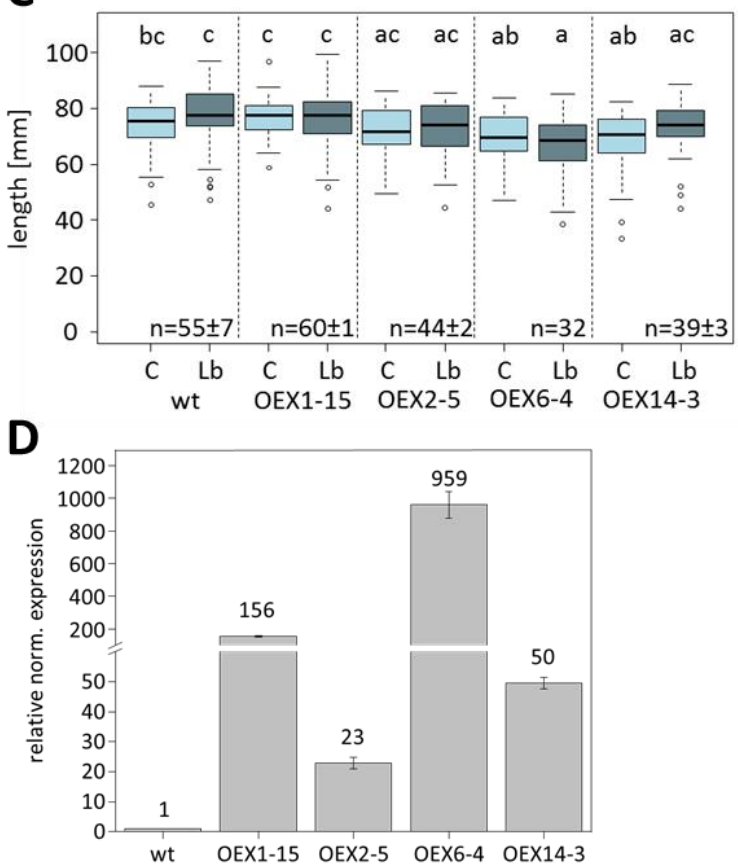

Figure 52. LR density of VALR-OEX lines. A) Typical examples of seedlings of VALR-OEX lines, of which the LR density was determined. For analysis, 6 petri dishes with 15 seeds per line and condition were prepared. B) LR density and C) primary root length of OEX-lines. Four-day-old seedlings were exposed to volatiles of L. bicolor for 7 days, according to the protocol in chapter 2.13. Since not all seeds germinated the number of analyzed plants per line varied between treatments and lines as indicated by $n$ in the figure. Data originated from one experiment. For statistical analysis, an ANOVA was performed. Different letters above the bars indicate significant differences at $p<0.05$, according to post-hoc Tukey's test. C, control; Lb, L. bicolor. D) Relative normalized VALR expression in roots of OEX-lines. Roots grown under control conditions from 2 petri dishes from one experiment were pooled for RNA extraction. qRT-PCR was performed as described in chapter 2.10.9. 


\subsubsection{GFP expression in VALR overexpression lines}

To obtain information on the localization of the protein we investigated the fluorescence signature in line OEX6-4 and line OEX14-3, which were expressing eGFP along with VALR (p35S::eGFP-At4g31330). We found the GFP fluorescence signal in the roots of the OEX lines (Figure 53). The signal was weaker in the cotyledons. In consensus with the expression level (Figure 52D), the OEX6-4 line showed the highest fluorescence intensity, although it varied from plant to plant. Compared to wildtype, OEX14-3 showed fluorescence in the roots as well (Figure 53).

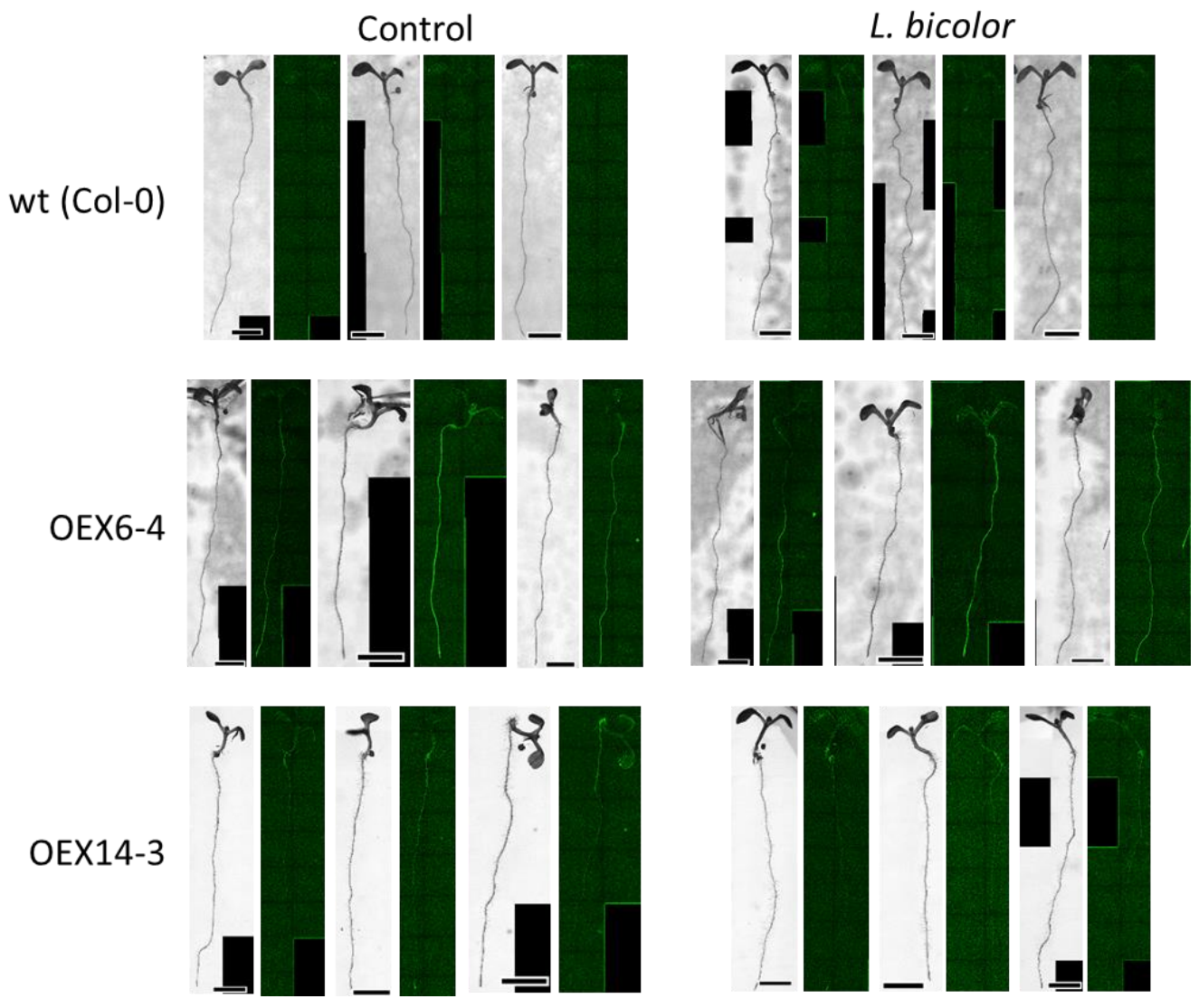

Figure 53. GFP fluorescence in VALR-OEX6-4 and OEX14-3 lines. Four-day-old seedlings were exposed to volatiles of $L$. bicolor for 2 days. Three seedlings per line and condition were investigated. For microscopy, seedlings were kept inside the petri dish (chapter 2.13). Fluorescence was detected with a stereomicroscope. Scale bar $=3 \mathrm{~mm}$. 


\subsection{Similarity of transcriptome data to published sequencing data}

Since our analyzes of ABA mutant lines and VALR lines did not reveal clear insights into the molecular response in volatile exposed $A$. thaliana and poplar plants, we compared our transcriptome data to omics data in literature from plants that were grown in root contact with ectomycorrhiza (Figure 54A-D).

Kaling and colleagues (2018) grew poplar in a sand/peat mixture either with or without fungal inoculum. After 24 days, leaves of those plants were harvested and RNA was extracted for transcriptome analysis (Kaling et al., 2018). Twenty-two DEGs overlapped with our A. thaliana transcriptomic data and 15 DEGs in poplar transcriptomic data (Figure 54A). Overlapping DEGs were also found in the sequence of mycorrhized fine roots with $P$. involutus, another ectomycorrhizal fungus (Luo et al., 2009).

Vishwanathan et al. (2020) analyzed the rosettes of Arabidopsis, which were grown in root contact with L. bicolor in a petri dish system (Vishwanathan et al., 2020). We found DEGs in Arabidopsis and poplar exposed to L. bicolor volatiles which were also regulated in rosettes of plants grown in contact with the fungus (Figure 54C). Felten et al. (2009) performed a similar experiment as Vishwanathan (2020) with poplar. They grew poplar in petri dishes in a sandwich system in root contact with L. bicolor. Also, in comparison to this set up, overlapping DEGs in Arabidopis and poplars were found (Figure 54D) (Felten et al., 2009). Recently, Miloradovic van Doorn and colleagues investigated the proteome of isoprene emitting poplar roots (Miloradovic van Doorn et al., 2020). We found overlaps in our transcriptome data as well. This indicates that isoprene might play a role in the molecular response to volatiles. 
A
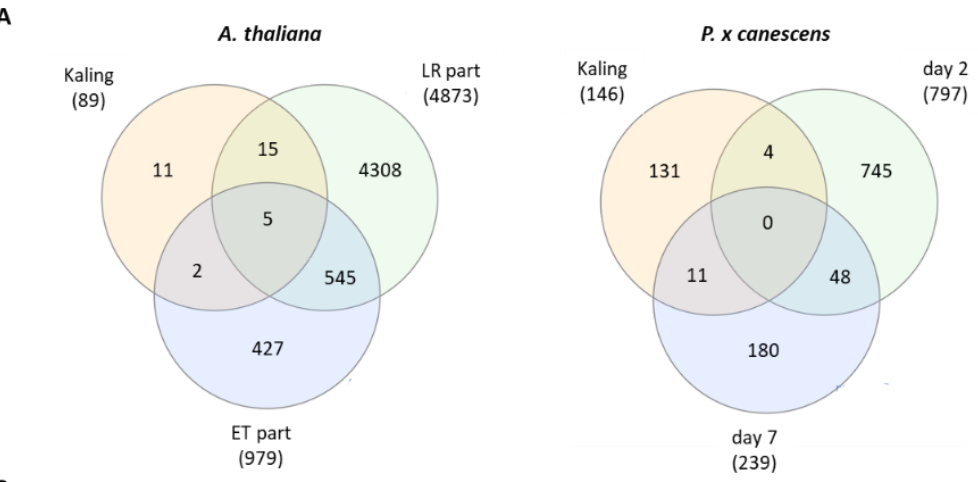

B
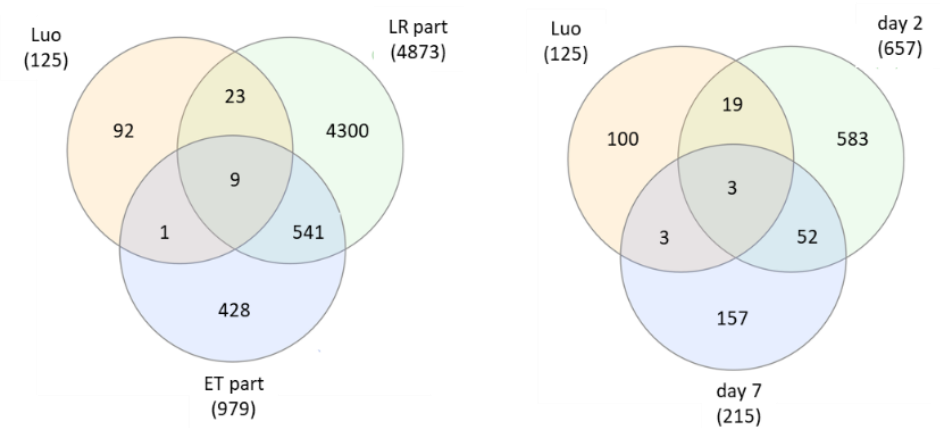

C Vishwanathan
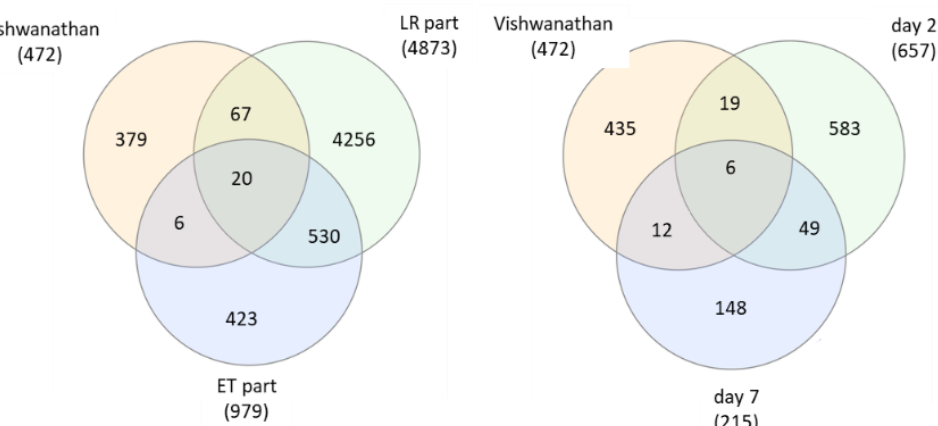

D
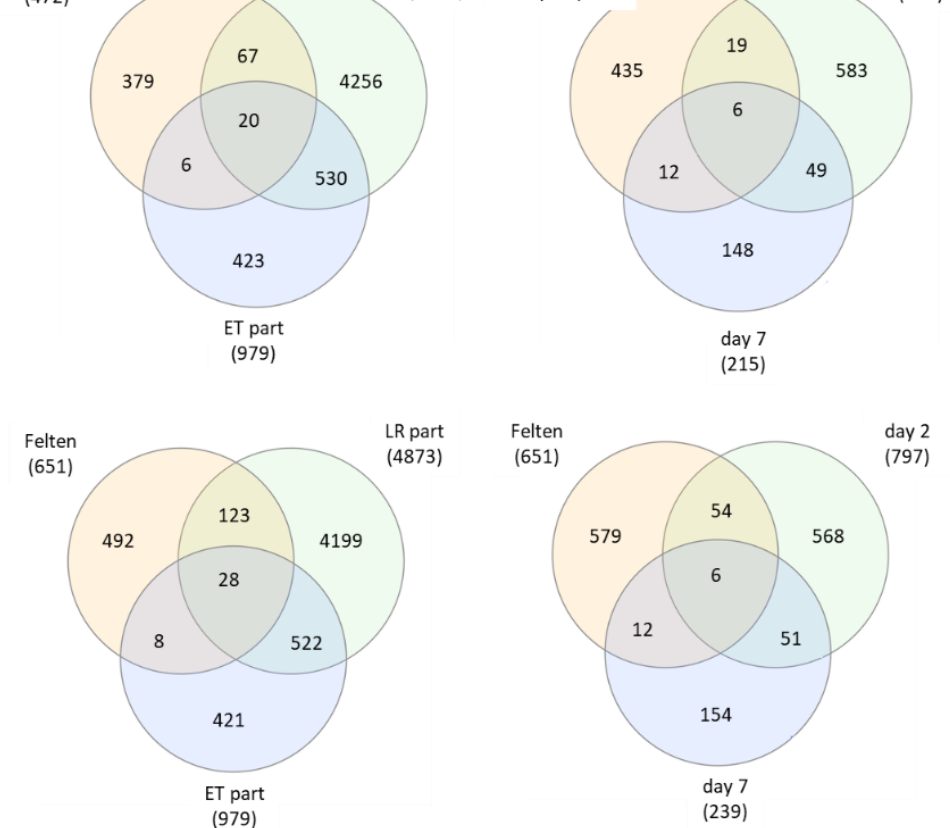

E
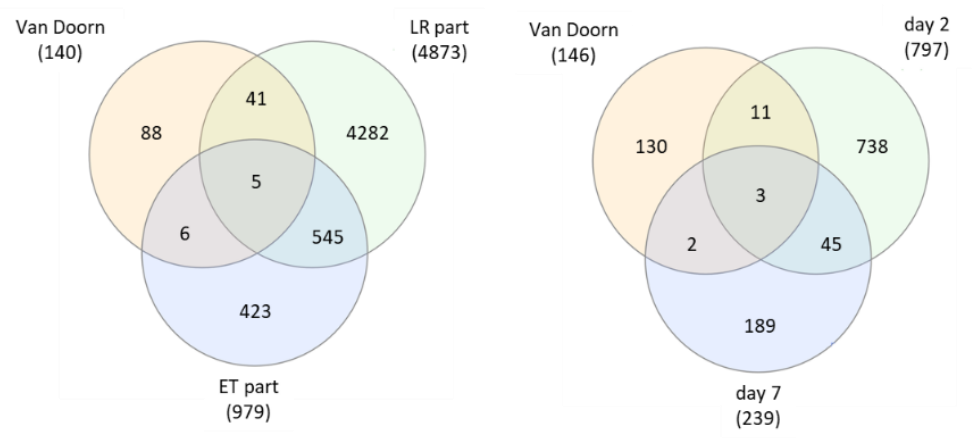

Figure 54. Legend on next page. 
Figure 54. Number of genes of $A$. thaliana and $P$. canescens roots found in literature of fungus-plant interaction. For $A$. thaliana Agi.ID were used for comparison and for P. $x$ canescens Potri.IDs. A) DEGs from Kaling et al. (2018), leaves from non-mycorrhized poplar vs. L. bicolor mycorrhized poplar (in contact), were compared to our transcriptome root data. B) DEGs found in Luo et al. (2009). Here, Agi.IDs were used for comparison for poplar as well. Luo et al. grew P. $x$ canescens plants in contact with $P$. involutus, an ectomycorrhizal fungus, and analyzed the changes in gene expression in fine roots. C) DEGs from Arabidopsis rosettes, whose roots were cultivated in contact with L. bicolor. D) Felten et al. (2009) analyzed the transcriptome of L. bicolor mycorrhized P. x canescens roots. E) DEGs compared to van Doorn et al. (2020). They analyzed proteome expression in roots of isoprene emitting poplar compared to non emitting poplar. (Felten et al., 2009; Kaling et al., 2018; Luo et al., 2009a; Miloradovic van Doorn et al., 2020; Vishwanathan et al., 2020). 


\section{Discussion}

\subsection{Role of CRKs (cysteine-rich receptor-like kinases) in volatile-induced LR formation}

CRKs play a role in different physiological processes and signaling networks in plant development, pathogen defense and abiotic stress responses. Some CRKs are affected by ROS (Bourdais et al., 2015; Idänheimo et al., 2014), which have been suggested to be involved in volatile perception (Ditengou et al., 2015). In this study, we investigated the role of CRKs in volatile-induced LR formation. For this purpose, we screened the crk T-DNA insertion library (Bourdais et al., 2015).

Since $A$. thaliana is not a host of L. bicolor, we confirmed protein homology of CRKs with those of $P$. trichocarpa by sequence alignment and generation of a phylogenetic tree. In consensus with Vaattovaara et al. (2019), we identified 64 CRK proteins in P. trichocarpa. In Arabidopsis, we identified the same CRK groups as Bourdais et al. (2015) (Figure 14) (Bourdais et al., 2015; Vaattovaara et al., 2019). However, we did not find homologous proteins in P. trichocarpa for the Arabidopsis groups III and IV. Since the groups are formed according to the intracellular kinase domain and the extracellular region, poplar CRK amino acid sequences for those domains might differ from the Arabidopsis CRK proteins of group III and IV.

For investigating the role of CKRs in response to volatiles in Arabidopsis roots, we exposed the T-DNA insertion lines of 42 of 44 members of the CRK family to volatiles of L. bicolor. Most of the mutant lines behaved like wildtype (Figure 15). However, crk3, crk4, crk42 and crk46 showed no significant different LR density between control and treated samples. These results indicate, that CRK3, CRK4, CRK42 and CRK46 might play a role in volatile-induced LR formation. However, we must admit, that the results originate from different experiments. Crk3 was investigated in experiment 3, crk4, crk42 and crk46 were investigated in experiment 5 and crk42 exposure was repeated in experiment 6 . Thus, only line $c k r 42$ was investigated twice, the other lines only once. Thus, the experiments with those lines should be repeated to confirm or disprove the results. 
We were especially interested in the crk29, crk28 and crk42 lines because those lines showed longer primary root length in the phenotypic analysis by Bourdais et al. (2015). In the transcriptome data of root parts (Table S4), we found a significant downregulation of CRK28 in the lateral root part of A. thaliana seedlings exposed to L. bicolor volatiles compared to non-treated plants. Furthermore, Pelagio-Flores et al. (2020) recently showed that crk28 formed more lateral roots compared to wildtype, while the CRK28 overexpression line formed fewer lateral roots, suggesting a negative regulating role of CRK28 in LR formation (Pelagio-Flores et al., 2020). Nevertheless, considering the LR density under control conditions, we did not find a difference to wildtype for $c k r 28$ and crk29. Reasons for the discrepancy to Pelagio-Flores et al. (2020) findings for crk28, might be the age of the plants and changes in the experimental set up. We used squared petri dishes, whereas Pelagio-Flores et al. (2020) used round petri dishes. They investigated 7-days-old seedlings while we analyzed 11-day-old seedlings. The main reason for the different phenotypes might be the medium. We used AM medium, which consists of $0.5 \times$ MS medium. Pelagio-Flores et al. (2020) in contrast, used $0.2 \times$ MS medium. Thus, nutrient concentration is reduced in this medium compared to our AM. Since crk28 is sensitive to abiotic and biotic stress (Bourdais et al., 2015) and since limited nutrient availability affects LR formation (Waidmann et al., 2020), the low concentrations of nutrients might have led to enhanced LR formation in crk28 in the study of Pelagio-Flores et al. (2020) (Pelagio-Flores et al., 2020).

Crk42 showed a significantly higher LR density compared to wildtype under control conditions, indicating a negative regulating role of CKR42 in LR formation. If this was the case, we expected an enhanced LR density in the crk42 line upon volatile exposure compared to wildtype. This was not observed. Thus, a function of CRK42 in LR remains unclear.

In summary, we could not find clear evidence that one of the CRK proteins is involved in volatile-induced LR formation in A. thaliana. However, a role of CRKs cannot be excluded, since they show high redundancy (Bourdais et al., 2015; Burdiak et al., 2015; Lu et al., 2016). Due to the high number of CRKs and missing clear evidence for an involvement in volatile-induced LR formation, we did not follow CRKs further on. 


\subsection{Role of peroxidases, germin-like proteins and strigolactones in response to volatiles}

With the aim to find potentially involved genes in volatile-induced LR formation, we screened the T-DNA insertion lines per45, per59 and arising from microarray data (Anna Schmidt, 2014, Table S2). Furthermore, we screened GLP3 T-DNA insertion and overexpression lines, as well as the strigolactones biosynthesis mutant max4-1 and the strigolactones signaling mutant max2-1. Strigolactones play a role in hormonal crosstalk during LR development and repress LR formation (Figure 17; Figure 18; Figure 19) (Kapulnik et al., 2011; Ruyter-Spira et al., 2011). The apoplastic location of GLP3 and the roles of germin-like proteins in pathogen defense indicate an involvement in response pathways to volatiles of L. bicolor (Floerl et al., 2012). None of the peroxidase, GLP3 or strigolactones mutants showed a different response to $L$. bicolor volatiles compared to wildtype. However, under control conditions, max4-1 showed reduced LR density compared to wildtype, which is contrary to our hypothesis that strigolactone deficient mutants form more lateral roots compared to wildtype. Furthermore, Ruyter-Spira et al. (2011) showed a 9\% higher LR density in max4 plants compared to wildtype under control conditions. Reasons, why max4-1 might have formed less LRs compared to wildtype under control conditions are diverse. One reason might be that MAX2, which acts downstream of MAX4, is induced by other substances than strigolactones as well. For example, glutathione affects root architecture in a MAX2 dependent manner (Marquez-Garcia et al., 2014) and karrikin induces MAX2 during seed dormancy (Nelson et al., 2011). Induction of MAX2 alters auxin transport (Ruyter-Spira et al., 2011), leading to reduced LR formation. This explanation is in consensus with the enhanced LR development in the max2-1 line (Figure 19). However, if MAX2 regulation was the reason for reduced LR density in $\max 4-1, \max 4-1$ and wildtype plants should have shown the same LR density under control conditions. Another explanation might be reduced phosphate levels.

Max4 mutant lines showed decreased LR formation compared to wildtype upon phosphate deficiency (Jiang et al., 2011). However, the phosphate reduced medium contained $10 \mu \mathrm{M}$ phosphate, while our AM medium contained $625 \mu \mathrm{M}$ phosphate. 
Thus, phosphate deficiency is probably no explanation for the reduced LR formation in max4-1. Taken together, the reasons for the reduced LR density in the max4-1 lines remain unclear.

\subsection{Reasons for the waved and aslant root phenotype in all experiments}

Throughout all experiments with Arabidopsis, we noticed that roots tend to grow to the right side on agar. This effect was most obvious with ecotype Ws-2 (Figure 18). Instead of growing straight to the bottom of the petri dish, roots formed waves and grew to the right-handed site (Figure 18). Also turning the petri dishes by $180^{\circ}$ around the vertical axis in the climate chamber led to the same effect, indicating no attraction to something in the climate chamber. The waved root growth is commonly observed. Simmons et al. (1995) and Rutherford and Masson (1996) reported aslant growth of Arabidopsis roots on agar surfaces. The effect was particularly clear in Wassilevskaja (Ws) and Landsberg erecta (Ler) ecotype and less common in Col-0. Our observations confirm these reports. Oliva and Dunand (2007) and Migliaccio et al. (2009) reviewed this phenomenon and suggested the presence and concentration of certain compounds in the medium as well as gravitropism, circumnutation and negative thigmotropism as possible reasons. Basically, Arabidopsis root tips touch the agar surface from time to time due to gravity. Touching the surface forces the tip to change growth direction, leading to waves. However, root waving and skewing was also observed in spaceflight experiments (Califar et al., 2020; Paul et al., 2012; Roux, 2012), suggesting directional light as a reason for directed growth (Paul et al., 2012; Roux, 2012). On the molecular level, auxin (Rashotte et al., 2000; Richter et al., 2009; De Smet et al., 2007), ethylene (Buer et al., 2003), cytokine (Kushwah et al., 2011), brassinosteroids (Lanza et al., 2012) and karrikin (Swarbreck et al., 2019) were involved in forming the waved root structure.

Although research on the waved root phenotype uncovered several insights into this phenomenon, the mechanisms behind it are poorly understood and need further research. 


\section{4 pGATA23::nls-GFP-GUS marker as a LR formation reporter line}

We tested, if the pGATA23::n/s-GFP-GUS marker was a suitable reporter line for volatileinduced LR initiation. We hypothesized that volatile-induced LR formation is causes by enhanced primordia formation. To analyze the primordia formation, we exposed the pGATA23::n/s-GFP-GUS line to L. bicolor volatiles and investigated the roots with a fluorescence microscope after 1, 2, 4 and 7 days of exposure.

The ideal case would have been, to see directly whether the treated roots showed more fluorescence signals, and thus more primordia, than the control roots. According to the figures in De Rybel et al. (2010) only the nuclei of primordia forming cells fluorescent. In contrast, we found that the fluorescence signal was not restricted to primordia forming cells (Figure 20). Thus, it was not possible to detect the primordia in a time-saving manner. Nevertheless, we counted the accumulation of fluorescent nuclei, which we defined as primordia and lateral roots (non-fluorescent). There was no difference in primordia density and LR density between volatile exposed plants and plants not exposed to volatiles. The LR density increased over time, whereas the primordia density decreased. This is logical since the primary root length increased over time while the number of primordia stayed the same.

Our results did not show a significant increase in primordia or lateral root density upon volatile exposure. However, we noted a trend after 4 and 7 days of exposure (Figure 20E). In contrast, pGATA23::n/s-GFP-GUS plants, which were not analyzed with the microscope, showed significantly enhanced LR density upon volatile exposure in comparison to control plants (Figure 20F). For testing, if pGATA23::n/s-GFP-GUS seedlings behave like wildtype under control conditions and upon volatile exposure, we determined the LR density of $73 \pm 2$ pGATA23::nls-GFP-GUS plants. For microscopical analysis, we could investigate only 5 plant, because the investigation was very timeconsuming.

The results showed that pGATA23::nIs-GFP-GUS seedlings responded to L. bicolor volatiles with enhanced LR density when investigating $73 \pm 2$ plants and that the microscopically analyzed root showed a trend towards enhanced LR and PR density after 4 and 7 days of exposure compared to control conditions. Thus, we assumed that too less plants were considered for LR and primordium density determination with the 
microscope to find a significant difference between treated and untreated that pGATA23::n/s-GFP-GUS seedlings.

With the aim to investigate the distribution the primordia forming cells in the root, we examined the pGATA23::n/s-GFP-GUS plants with a stereomicroscope which allowed us to investigate the same plants at different time points and to take picture from whole roots. The fluorescent signal was located in the lateral root initiation zone, between the youngest lateral root and the root tip. Since this is the location for primordia formation, we expected the fluorescence signal to be there. However, we also noticed enhanced GFP expression in curves of the primary roots. This finding suggests primordia and LR development in the curves of the primary root. This finding is in consensus with other publications, in which LR development in root curves was observed (Ditengou et al., 2008; Fortin et al., 1989; Laskowski et al., 2008; Lucas et al., 2008; Richter et al., 2009; De Smet et al., 2007). Based on the agravitropic auxin transport mutant aux1, which turns only in one direction, De Smet et al. (2007) showed that more LRs emerged on the outside of the curved roots. This finding suggests that LR formation in root curves is associated to the gravity response via auxin transport. Further studies of these processes were performed by inducing root bending by mechanical methods and gravistimulation (Ditengou et al., 2008; Laskowski et al., 2008; Lucas et al., 2008; Richter et al., 2009). These studies showed that roots defective in gravity sensing, still form LRs on the outside of the primary root curves (Ditengou et al., 2008; Lucas et al., 2008; Richter et al., 2009). Furthermore, in spaceflight experiments lateral roots grew at the curved side at the root as well (Paul et al., 2012). Thus, the LR development in root curves is gravity independent.

Besides changes in the expression and localization of auxin reporters after mechanical induction of bends (Ditengou et al., 2008; Laskowski et al., 2008), rapid changes in calcium $\left(\mathrm{Ca}^{2+}\right)$ levels were observed (Richter et al., 2009).

Treatment with calcium channel blockers inhibited changes in calcium level and primordium development. These results suggested, that $\mathrm{Ca}^{2+}$ signaling is involved in $\mathrm{LR}$ formation in root curves (Richter et al., 2009).

The results from the mentioned studies highly suggest, that the pGATA23::nls-GFP-GUS primordia line marker showed enhanced fluorescence in the curved part of the root due to initiation of LR development. 


\subsection{Volatile induced LR formation is dose- and time dependent}

A main goal of this thesis was to uncover the molecular responses downstream to volatile perception in Arabidopsis and poplar. For this purpose, it was crucial to find out whether the volatile-induced LR formation was dose- or time dependent. We showed enhanced LR density in Arabidopsis roots co-cultivated with the mycelium of one fungal plug, indicating that the volatiles emitted by this amount of mycelium is sufficient to induce the volatile-induced LR formation in Arabidopsis. With the mycelium of two L. bicolor plugs LR densities increased further but additional fungal plugs did not stimulate extra LRs. Thus, the root response to L. bicolor volatiles is dose-dependent but only to a certain volatile concentration level. A reason for the saturation could be repletion on volatile receptors. This conclusion presumes that the higher the number of fungal plugs and hence, the larger the mycelium is, the higher is the amount of emitted volatiles.

Weikl et al. (2016) showed that volatile emission of Alternaria alternate and Fusarium oxysporum decreased with fungal age (Weikl et al., 2016). If this was also true for L. bicolor the emitted volatile concentration level might have decreased with increasing plug age. This might also be the reason for the stronger increase in LR density in Arabidopsis grown with 9-day-old fungi compared to 18-day-old fungi (chapter 2.2.1). On the other hand, (-)-thujopsen, a sesquiterpene, which was isolated from L. bicolor and induced LR formation in Arabidopsis and poplar, was applied to the roots in concentrations ranging from 0 to 1000 ppb (Ditengou et al., 2015). The higher the concentration was, the higher was the number of lateral roots. At 100 ppb, a significant difference in LR number between control and treated roots was seen. If we assume higher volatile concentration, and thus higher (-)-thujopsen concentrations in the headspace of the petri dish with increasing number of fungal plugs, the LR density should have consistently increased with the amount of fungal plugs. This was not the case for 2, 3 and 4 fungal plugs. Possible explanations for this observation might be either that the (-)-thujopsen concentrations used by Ditengou et al. (2015) did not replete the assumed receptors or that or volatile emission of $L$. bicolor decrease over time. Another possibility is that the number of primordia and LRs is limited, for example due to the number of xylem pole pericycle cells and the well-regulated auxin transport and 
signaling during LR formation. If this is the case, the amount of volatiles emitted by two fungal plugs might be sufficient to reach the maximal LR density.

Further research, for example determining the volatile concentrations of different number of fungal plugs over time, is necessary to solve this question.

We investigated, if a volatile pulse is sufficient to induce volatile-induced LR formation and to find a suitable volatile exposure duration for harvest transcriptome samples. We found that 2 days of exposure to $L$. bicolor volatiles were sufficient to induce the volatiledependent LR formation. Furthermore, the response was detectable 5 days after fungus removal, indicating that an initial signal is sufficient to induce LR formation. However, the longer the exposure, the higher was the LR density difference between control samples and treated samples. This result suggests that the molecular response leading to LR formation is enhanced the longer the plant is exposed to volatiles. In terms of ecology, this would make sense for L. bicolor host plant interaction. L. bicolor profits from the larger root surface of its host plant that is produced by enhanced LR formation, because it has more opportunities to get in contact with the plant to form a larger Hartig net for carbohydrate gain and nutrient supply.

We showed that LR formation in A. thaliana roots is time-dependent and that a volatile exposure pulse for two days is sufficient to induce the molecular responses leading to enhanced LR density. Previously, a significant difference in LR density between fungal-treated and non-treated Arabidopsis plants occurred after 7 days of exposure (Ditengou et al. 2015). In those experiments the fungus was not removed from the petri dishes.

A reason for the time lag between the current and the previous response time, might be differences in growth temperatures. Ditengou et al. (2015) performed their experiments at $21^{\circ} \mathrm{C}$, while we used $22{ }^{\circ} \mathrm{C}$ during darkness and $24^{\circ} \mathrm{C}$ during light phase, which leads to a faster growth (ftp://ftp.arabidopsis.org/ABRC/abrc plant growth.pdf, 2020). The slower growth of plants in Ditengou et al. (2015) is supported by the fact that we had to stop our experiments after 7 days of exposure with 11-day-old plants, because the roots where close to the Teflon barrier, while Ditengou et al. (2015) were able to examine 15-day-old plants in petri dishes. 
In conclusion, the L. bicolor volatile-induced LR formation in A. thaliana is activated in a dose and time-dependent manner.

\subsection{L. bicolor volatiles alter gene expression in several processes}

For investigating the molecular responses to volatile signaling in roots, we analyzed the transcriptome of $A$. thaliana and P. $x$ canescens roots exposed to $L$. bicolor volatiles. We showed that the LR density of $A$. thaliana seedlings increased during one week of exposure to L. bicolor volatiles. We found 44 DEGs in this time period, of which VALR (At4g31330, Protein of unknown function DUF599) was negatively regulated after 2, 4 and 7 days of exposure. The LR density of Arabidopsis roots showed the earliest significant increase after two days of exposure compared to non-treated roots.

This suggests that changes in gene expression are induced before 2 days of exposure. At 2 days of volatile exposure the transcriptome analysis showed the majority of DEGs. In a second experiment, we tested whether gene expression in the LR forming part of the roots differs from the elongation-tip part. Due to the former results, indicating changes in gene expression after 2 days, we analyzed the transcriptome of root parts and whole roots harvested after two days of volatile exposure. This time, we found only 7 DEGs in whole roots. Surprisingly, we found 4873 DEGs in the LR forming part and 979 DEGs in the elongation tip part. Possible explanations for the discrepancy of number of DEGs might be that changes in gene expression are too low to detect in whole roots or only happens in some cells, e.g. in LR developing cells during emergence and root apical meristem activation (Figure 1), which occur in the LR part of the root in an increased number. Furthermore, priming and founder cells formation happens in the elongation-tip part in only a few cells (Figure 1). These responsible changes in gene expression upon volatile exposure might not be detectable in whole root samples as well.

Since LR formation is associated with enhanced changes in gene expression (Brady et al., 2007; Fukaki and Tasaka, 2009; De Smet et al., 2008), a higher number of DEGs in the LR part of the was expected. However, why the changes in transcript expression are only detectable in the LR and in the elongation-tip part and not in the whole root, is not fully explainable. 
MapMan analysis revealed significantly enriched abundance of transcripts associated with protein metabolism (activation, synthesis, targeting, degradation and posttranslational modification), RNA (processing and regulation of transcription), cell wall, microRNA, photosystem, mitochondrial electron transport and ATP synthesis and glycolysis in Arabidopsis LR and elongation-tip part. We found downregulated DEGs involved in photosystem of Arabidopsis roots. This might be due to the COP1/DET1-mediated repression of chloroplast formation (Lau and Deng, 2012). COP1-and DET1 belong to a group of genes termed Constitutive Photomorphogenic/Deetiolated/Fusca (COP/DET/FUS) (Schwechheimer and Deng, 2000; Serino and Deng, 2003). Nine of the COP/DET/FUS loci encode COP1, DET1, COP10 and COP9 signalosome (CSN) subunits 1 to 4, 7 and 8. Indeed, our transcriptome data revealed upregulation of some of the COP/DET/FUS loci in the LR part of roots exposed to L. bicolor volatiles and downregulation of COP10 (Table DS1_Supplemental Word-File). These findings indicate enhanced suppression of photomorphogenesis in LR part of volatile exposed roots.

In leaves, $A$. thaliana plants exposed to microbial volatiles showed increased chlorophyll contents and photosystem efficiency (Hung et al., 2013; Lee et al., 2016; Minerdi et al., 2011; Xie et al., 2009; Zhang et al., 2009). Arabidopsis plants exposed to volatiles of Bacillus subtilis (GB03) increased total chlorophyll content to over $60 \%$ compared to the control plants. In addition, the photosystem II photosynthetic efficiency was increased (Xie et al., 2009). Zhang et al. (2009) tested the same interaction and found a chlorophyll increase of $84 \%$ compared to control plants (Zhang et al., 2009). Also, volatiles released by the fungus Fusarium oxysporum MSA35 increased chlorophyll content by $68 \%$ in leaves of lettuce (Minerdi et al., 2011). Volatiles of Trichoderma viride increased chlorophyll concentration by 58 \% (Hung et al., 2013). Lee et al. (2016) exposed Arabidopsis seedlings to volatiles of Triochderma species and found an increase of 82.5 to $89.3 \%$ in chlorophyll content (Lee et al., 2016). Furthermore, Arabidopsis exposed to volatiles of Alternara alternate or Penicillium aurantiogriseum volatiles showed enhanced chlorophyll content (García-Gómez et al., 2019). All these results suggest that microbial volatiles increase photosynthesis activity in leaves. Since roots do not contain chloroplasts, microbial volatiles cannot affect chlorophyll content in roots. 
However, there are other genes like the COP/DET/FUS-related genes, which suppress chloroplast formation in roots. Microbial volatile might induce the expression of photosystem-related genes. In leaves, this might lead to enhanced chlorophyll production, while in roots, chloroplast suppressing genes might be induced. Our results suggest that volatiles released from L. bicolor led to suppression of photosystem-related genes in A. thaliana roots.

In poplar, roots exposed to volatiles for 2 days showed downregulation of photosystem-related genes, but the COP/DET/FUS-related genes were not found (Table S5).

Our transcriptome data of $A$. thaliana root parts also show enriched amounts of upregulated transcripts related to glycolysis in LR parts of roots. Increased glycolysis might result in increased pyruvate production. Pyruvate might be transported in the mitochondrion and used in the citric acid cycle to generate ATP (adenosine triphosphate). Enzymes of the citric acid cycle, for example 2-oxoglutarate dehydrogenase, succinate dehydrogenase and malate dehydrogenase, are also significantly upregulated in the LR part of Arabidopsis. The production of ATP could explain the enriched transcript abundance in mitochondrial electron transport and ATP synthesis. ATP is needed for several energy consuming processes in the plant such as cell growth and development. One reason for enhanced ATP production might be that the increased LR formation upon volatile exposure might enhance the energy and metabolite demand. In addition, ATP potentially regulates defense response of plants to biotic and abiotic stress (Cao et al., 2014; Jia et al., 2016). Since many genes were found in these categories as well (Figure 30). ATP might also play a role in signaling processes induced by the volatile in plants.

Transcripts assigned to cell wall proteins, cellulose synthase and pectin esterases were enriched in lateral root parts of volatile exposed plants as well. This can be explained by the increased LR formation upon volatile exposure, for which cell expansion was needed. Furthermore, in a transcriptomic analysis of $A$. thaliana exposed to Bacillus subtilis (GB03) volatiles, Zhang et al. (2007) found regulated genes associated with cell wall proteins as well (Zhang et al., 2007). In Arabidopsis plants exposed to Bacillus amyloliquefaciens FZB42 volatiles genes associated to cell wall modifications 
were also differentially expressed (Hao et al., 2016). In more detail Zhang al (2007) and Hao et al., 2016 found pectin-related genes, such as pectate lyase. This group of genes was also found to be differentially expressed in our transcriptome data. Pectate lyase catalyzes the eliminated cleavage of de-esterified pectin (Carpita and Gibeaut, 1993). Pectins are suggested to have several cell wall related functions (Buchanan et al., 2000). One of them is to serve as a recognition molecule that alert plant cells in presence of symbiotic organisms, pathogens and insects (Buchanan et al., 2000).

Here we demonstrated that poplar roots, which were exposed to L. bicolor volatiles for 2 days, also showed significantly enriched cell wall related genes. These findings suggest that microbial volatiles, including the volatiles released by L. bicolor, are implicated in cell wall related gene expression and thus cell wall development.

In A. thaliana LR parts, genes of the microRNA, natural antisense etc. MapMan categories were enriched as well. MicroRNAs and natural antisense transcripts are known to play a role in post-transcriptional gene expression (Barciszewska-Pacak et al., 2015; Borsani et al., 2005; Grennan, 2008; Guo et al., 2005; Robinson and Parkin, 2008; Yuan et al., 2015). Hence, enrichment of related transcripts might be a hint, that molecular responses to $L$. bicolor volatiles are regulated on the post-transcriptional level. This suggestion is supported by the enriched transcripts related to RNA processing and regulation of transcription.

Since we also found enriched transcripts related to protein modification, synthesis, targeting, and degradation and folding, a post-translational regulation of proteins is also conceivable.

The global transcription analysis showed that exposure to fungal volatiles led to a pulse to increase LR growth during the first 2 days, which was achieved in the plants grown under control conditions to a later time point. Therefore, we did not find many differentially expressed genes in A. thaliana roots at later time points. This pulse induced changes in gene expression related to photosystem, minor carbohydrate (CHO) metabolism, cell wall, lipid metabolism, amino acid metabolism, secondary metabolism, hormone metabolism, stress, redox, RNA, protein, signaling, development, and transport in Arabidopsis roots as well as in poplar roots. 
Besides the roots, we analyzed the transcriptome of Arabidopsis rosettes after 4 days of exposure but we did not find any DEGs. This result might be caused by choosing a non-suitable day of harvest. The results of the Arabidopsis root transcriptome data and the response time suggest that a pulse before 2 days of volatile exposure is sufficient to induce transcriptional changes in the $A$. thaliana genome. Consequently, harvesting rosettes after 2 or 3 days of exposure might lead to a different result.

Furthermore, we also analyzed the transcriptome of poplar leaves after 2 and 7 days of exposure. We identified 100 DEGs in samples after 2 days exposure and 191 in samples that were exposed for 7 days. For poplar, the time of harvest did not seem to have an impact on the transcriptome. Perhaps the volatiles are perceived by the poplar leaves as it was shown for Arabidopsis leaves exposed to diverse volatiles and Arabidopsis shoots exposed to 1-decene from Trichoderma (Lee et al., 2019; Naznin et al., 2014). Mapping to MapMan Bins did not reveal an enrichment in any category and we could not find any ABA related gene.

\subsection{Role of ABA in volatile-induced $L R$ formation}

In order to uncover downstream responses of volatile-induced signaling in roots, we compared common hormone signaling pathway related genes to our transcriptome data of poplar and Arabidopsis (Figure 37, Table S9). Thereby, the highest abundance of DEGs were related to $A B A$ signaling. Besides the role of $A B A$ in abiotic and biotic stress (Chan, 2012; Fujita et al., 2006; Hewage et al., 2020), ABA is also involved in lateral root development (Fukaki and Tasaka, 2009). While ABA signaling mediated by ERA1 and $A B I 3$ is necessary for auxin-mediated LR formation, probably during LR initiation (Brady et al., 2003), exogenous ABA inhibits LR formation (De Smet et al., 2006). Furthermore, Zhang et al. (2008) reported, that volatiles released by Bacillus subtilis GB03 decreased ABA levels in foliar parts of Arabidopsis plants. However, they also showed, that this effect was not observed in roots (Zhang et al., 2008).

We showed that the ABA-related signaling components PYL1, PYL4, PYL8 as well as $A B A 1$, which plays a role in $A B A$ biosynthesis were downregulated in $L R$ parts of Arabidopsis. Furthermore, the transcription factor MYB77, which activates auxin-inducible genes in the absence of exogenous auxin by forming a heterodimer with 
ARF7 (Shin et al., 2007; Zhao et al., 2014), was upregulated in upon volatile exposure of Arabidopsis. Interaction of MYB77 with PYL8 was shown by yeast-two-hybrid assays (Zhao et al., 2014). Besides, the pyl8-1 mutant was insensitive to exogenous ABA in terms of root length and LR number (Xing et al., 2016; Zhao et al., 2014). Upon ABA treatment, the roots were longer and the LR number higher compared to wildtype (Xing et al., 2016; Zhao et al., 2014). In poplar roots, the PP2C genes HIGHLY ABA INDUCED HAI2 and HAI3 were downregulated (Table S7). These genes play a role in water-related stress (Bhaskara et al., 2012; Fujita et al., 2009) and interact with PYL8 (Bhaskara et al., 2012). These results suggest that, PYL8 negatively influences LR formation. The downregulation of $A B A-r e l a t e d$ genes is in consensus with the finding that $A B A$ inhibits LR emergence (De Smet et al., 2003). Thus, ABA-related genes were potentially involved in volatile induced LR formation.

L. bicolor volatile treatment of $A B A-s y n t h e s i s$ and signaling mutants revealed an increased LR density in aba2-1 compared to wildtype but reduced LR response to volatiles in aao3-4 and py/8-1 (Figure 39). Since the transcript abundances of these genes were downregulated (PYL8) or tended to be decreased upon volatile exposure (ABA2 and $A A O 3$ ) we expected increased LR density in the knockdown mutants compared to wildtype if exposed to L. bicolor volatiles. ABA synthesis is reduced by 20 to $25 \%$ in the aba2-1 mutant compared to wildtype (Gonzalez-Guzman et al., 2002). Since the difference in LR density of aba2-1 seedlings grown under control condition and exposed seedlings is higher compared to the difference in wildtype plants, it is likely that ABA negatively influences LR formation. On the other hand ABA synthesis in aao3-4 is reduced by 50 to $60 \%$ (Seo et al., 2004) and this line showed no significant difference in LR density between the control seedlings and the volatile exposed seedlings. Therefore, the role of $A B A$ synthesis in volatile induced $L R$ formation is not clear.

Contrary to our expectation, py/8-1 showed lower increase in LR density upon volatile exposure than the wildtype. Since ABA-treatment leads to increased LR number in py/8-1 (Zhao et al., 2014), volatile mediated reduced ABA concentrations might have the opposite effect on LR formation, leading to decreased LR formation. Reduced ABA concentrations might abolish the inhibiting effect of ABA on LR emergence. 
We observed that the sextuple pyr1 pyl1 py/2 pyl4 pyl5 pyl8 mutant tented to show a higher difference in LR density between the control roots and the volatile exposed roots compared to wildtype. This effect supports the assumption that ABA is reduced upon L. bicolor volatile treatment. To obtain further support for the assumption that ABA concentrations play a role in volatile-mediated LR induction, phytohormones should be analyzed in future experiments.

Myb77-1 behaved like wildtype upon fungal volatile treatment. Thus, an involvement in response to volatiles is unlikely.

In addition to the tested mutant lines, we found different gene expression in members of the cytochrome family P450, which helps to protect the plants from stress. Two of the members, CYP707A1 and CYP707A3 were upregulated in the lateral root parts of Arabidopsis plants treated with L. bicolor volatiles. CYP707As catalyze the ABA 8'-hydroxylation reaction in ABA catabolism (Kushiro et al., 2004; Saito et al., 2004). Thereby, ABA concentration in the cells might be reduced leading to the abolishment of inhibited LR emergence.

Overall, our study suggests that L. bicolor volatile-mediated downregulation of ABA synthesis and signaling might be involved in the abolishment of the inhibition of LR emergence. Thus, volatile-exposed plants can develop more lateral root per root length. However, this suggestion needs to be validated by e.g. repeating the experiment with aba2-1, aao3-4, pyl8 and the pyl sextuple mutant, because data from the sextuple mutant and aao3-4 are currently based on only one experiment. Furthermore, determination of $A B A$ content in the roots might be helpful to analyze the role of $A B A$ in volatile-induced LR formation.

\subsection{Role of VALR in volatile-induced LR formation}

We identified VALR (At4g31330, Protein of unknown function, DUF599) in the transcriptomic data of Arabidopsis roots harvested after 2, 4 and 7 days of volatile exposure. Since this was the only gene, whose transcripts were consistently regulated during the whole time-period, it was chosen as a candidate for in-depth analyses. Further transcriptomic data in LR parts corroborated the downregulated expression of VALR upon volatile exposure. 
We confirmed this by investigating the VALR expression via qRT-PCR as well. Furthermore, the poplar homolog of VALR (Potri.006G278000.1) tended to downregulation according to the transcriptomic data of poplar roots as well. In silico studies, which showed VALR expression in the pericycle of roots and downregulation upon LR formation supported our assumption that VALR might play a role in volatile-induced molecular responses. VALR contains four predicted transmembrane domains, indicating a membrane-localization. Thus, it could serve as a receptor or transporter for substances affected by L. bicolor volatile exposure.

VALR-T-DNA insertion lines showed reduced response to volatiles, compared to wildtype plants, although we expected an enhanced response. qRT-PCR revealed that the T-DNA insertion lines overexpress the VALR gene, instead of suppressing it. This is probably due to the position of the T-DNA in front of the gene, which could lead to an activation. The T-DNA contains a constitutive $35 \mathrm{~S}$ promotor which controls expression of the resistance genes. If the T-DNA is inserted in front of a gene, it is possible that the $35 \mathrm{~S}$ promotor also controls expression of the gene of interest, leading to overexpression (Ülker et al., 2008).

Knowing, that VALR was overexpressed in the T-DNA insertion lines, the reduced increase in LR density upon volatile exposure corresponded to our hypothesis that VALR is involved in the molecular response to volatiles.

We hypothesized that volatile exposure leads to downregulation of $V A L R$, which results in increased LR formation. To test this hypothesis, we created loss-of function valr mutants using CRISPR/Cas9. Consequently, we expected an increased LR development under control conditions, if VALR played a role in LR formation in general or a higher increase compared to wildtype upon volatile exposure, if $V A L R$ plays a role in volatile-mediated LR formation. Although, we confirmed the VALR suppression in lines valr4-8, valr6-1, valr1-5-1 and valr1-5-9, a clear difference in response to L. bicolor volatiles compared to wildtype plants was not detected. The valr suppression mutants tended to develop less LRs than wildtype plants in the absence of volatiles (Figure 48). This is contrary to our hypothesis that VALR downregulation increases LR formation. Perhaps, the LR formation is VALR dose-dependent. In that case, higher accumulation of VALR might lead to suppression of LR formation as well as too low concentration. 
However, based on the results of the VALR-OEX lines exposed to L. bicolor the latter hypothesis has also to be rejected. The VALR OEX lines OEX1-15, OEX2-5, OEX6-4 and OEX14-3 behaved like the wildtype upon volatile exposure. The VALR-OEX lines tended to have shorter primary roots. In addition, VALR-OEX lines seemed to have a lower germination rate compared to wildtype seedlings. VALR-OEX6-4 showed the highest VALR expression and the lowest number of analyzed plants (Figure 52). Thus, VALR might play a role in germination and primary root growth. The role in root growth is supported by the microscopic results of eGFP expressing VALR-OEX lines. We found the fluorescence signal predominantly in the roots, even though the p35S-promotor should be active in all tissues. The fluorescence proved successful transformation on the one hand, and on the other hand localized the protein in the roots. One reason for nonfluorescing leaves in the $p 35 S:: G F P-V A L R$ line might be, that the protein is degraded in the leaves. Reasonable reasons for this are unknown.

Investigation of VALR-OEX6-4 with a confocal microscope can provide further insights into the role of VALR in terms of its subcellular location.

In conclusion, a specific role in volatile-induced LR formation was not demonstrated here. Further investigation and repetition of the experiments are necessary to validate these results, which are currently based on only one experiment.

\subsection{Overlaps of transcriptome data and published sequencing data}

Since our analyzes of ABA mutant lines and VALR lines did not reveal clear insights into the molecular response in volatile exposed $A$. thaliana and poplar plants, we compared our transcriptome data to omics data in literature from plants that were grown in root contact with ectomycorrhiza (Figure 54A-D).

Among the DEGs, which overlapped with the data from Kaling et al. (2018) and Luo et al. (2009), who analyzed poplar mycorrhized roots, we found RD26. RD26 encodes a NAC transcription factor in response to drought. Under drought, ABA expression leads to induction of BIN2, which phosphorylates RD26, leading to an increased stress response (Jiang et al., 2019). Thus, RD26 positively regulates ABA-related stress responses. Since RD26 was downregulated in A. thaliana and P. $x$ canescens roots exposed to L. bicolor volatiles, we assume that $A B A$ related gene responses are downregulated. 
This might help the fungus to overcome potential defense responses or release the inhibition of LR emergence.

Vishwanathan et al. (2020) analyzed the rosettes of Arabidopsis grown in root contact with $L$. bicolor. Interestingly, VALR was among the overlapping genes in leaves, but it was upregulated. Thus, VALR seem to be upregulated in leaves upon L. bicolor root contact and downregulated in roots exposed to L. bicolor volatiles. We could not see a connection between both regulations.

Miloradovic van Doorn et al. (2020) investigated the proteome of isoprene emitting poplar roots. Overlaps with these data indicates that isoprene might play a role in the molecular response to volatiles. Isoprene improves plant tolerance against abiotic and biotic stress (Vickers et al., 2009). In addition, isoprene reacts with reactive oxygen and alters ROS signaling indirectly (Affek and Yakir, 2002; Vanzo et al., 2016; Velikova et al., 2012, 2004).

Recently, Xu et al. (2020) showed that overexpression of isoprene synthase altered ABA-related gene expression which leads to higher tolerance to abiotic stress. (J. Xu et al., 2020). For this reason, in poplar plants isoprene emission might be involved in the response to L. bicolor volatiles. However, it is synthesized in chloroplasts, and thus in leaves of plants. Therefore, it needs to be transported or perceived by the roots to affect $A B A$ or ROS signaling there. 


\section{Conclusion}

During the last years, research on volatile-related effects on plants got increasing interest, due to the role of volatiles in supporting symbiotic plant-microbe interactions and their potential to reduce abiotic and biotic stresses. Ditengou et al. (2015) showed that volatiles released by the ectomycorrhizal fungus L. bicolor led to an increased number of lateral roots in its host plant $P$. $x$ canescens but also in the model plant A. thaliana. They also showed that ROS accumulated in roots, which were exposed to L. bicolor volatiles. The aim of this study was to uncover the molecular responses behind the induced LR formation. We showed that the LR response in A. thaliana to volatiles was dose-dependent, but when a threshold is reached, LR density did not increase anymore. We also showed that already 2 days of volatile exposure induced increased LR formation. Since the increase could be observed several days after fungal removal, our results indicate that molecular processes once induced are kept active until LR formation.

Our study provides the first transcriptomic data of plants exposed to volatiles of L. bicolor. We harvested the samples for transcriptome analyses in a time-dependent manner and found most of the DEGs in roots after 2 days of volatile exposure, suggesting that molecular changes are induced during this time. We found a yet uncharacterized protein, VALR (At431330; Protein of unknown function), to be downregulated after 2, 4 and 7 days of exposure. Since this was the only regulated gene in roots at day 4 and 7 , we started functional analyses. Nearly nothing is known about this gene. In silico analysis showed that VALR is expressed in the pericycle and downregulated upon LR formation. VALR was also downregulated in Arabidopsis roots exposed to L. bicolor volatiles. We found that LR density in the valr loss-of-function mutants varied between wildtype behavior and reduced response to volatiles. The VALR overexpression lines behaved like wildtype. Therefore, VALR involvement in volatile-induced LR formation is doubtful. However, since the experiments were only performed once due to time limitations, independent replications are required.

In the transcriptomic data of $A$. thaliana root parts and $P$. canescens roots, we found evidence for $A B A$ to play role in volatile induced-LR formation. ABA-related genes were downregulated in both plant species. 
This was surprising, since ABA supports LR initiation in an auxin mediated manner (Brady et al., 2003). However, exogenous ABA inhibits LR emergence (De Smet et al., 2006). We exposed knockdown mutants for ABA synthesis (aba2-1 and aao3-4) and signaling (py/8-1, pyl sextuple mutant and myb77-1) to L. bicolor volatiles and found that the increase in LR density was enhanced in the synthesis mutant aba2-1 and tended to be increased in pyr1 pyl1 pyl2 pyl4 pyl5 pyl8 mutant line. Furthermore, we found genes downstream of $A B A$ that are responsible for $A B A$ catabolism (upregulated) or activation of $A B A$-related stress responses (down regulated). These results led us to the suggestion that L. bicolor volatiles reduced ABA concentrations to abolish the inhibition of LR emergence.However, the variety of genes regulated in the transcriptomic data of Arabidopsis root parts and the poplar roots indicated that the volatile-induced LR formation is not limited to one molecular response or signaling pathway. Especially in lateral root parts, of the root, we found genes involved in other phytohormone-related processes, like JA, auxin transport and ethylene. We also found ROS-related genes in poplar and Arabidopsis, confirming that ROS plays a role in volatile induced LR formation (Ditengou et al., 2015). Further research needs to be done to identify the complex regulation of volatile-induced $L R$ induction.

Besides the roots, we analyzed the transcriptome of Arabidopsis rosettes after 4 days of exposure and poplar leaves after 2 and 7 days of exposure as well. In Arabidopsis rosettes we did not find any DEGs. This result might be caused by choosing a non-suitable day of harvest. In poplar leaves, we found DEGs, but none of them was associated with ABA signaling. Thus, the responses in roots might be affect other processes in leaves or the volatiles are perceived in leaves as it was shown for other studies (Lee et al., 2019; Naznin et al., 2014). This question could be solved by an experiment, in which the roots of plants are separated from the shoots, e.g. by putting the roots in a small petri dish, close it dense, and putting the small petri dish with the plant in a bigger petri dish. Thus, the fungus could be either added to the roots or to the foliar plant parts.

Our results provide the start of research on the molecular effects of L. bicolor volatiles on roots of plants. Understanding the molecular processes is useful for further research on volatiles. Knowing the genes involved in the molecular responses enhance the chance 
to identify marker genes or proteins, which can then be used to study the effect of single isolated volatiles. 


\section{References}

Affek, H.P., and Yakir, D. (2002) Protection by isoprene against singlet oxygen in leaves. Plant Physiol. 129: 269-277.

Akiyama, K., Matsuzaki, K.I., and Hayashi, H. (2005) Plant sesquiterpenes induce hyphal branching in arbuscular mycorrhizal fungi. Nature. 435: 824-827.

Alder, A., Jamil, M., Marzorati, M., Bruno, M., Vermathen, M., Bigler, P., Ghisla, S., Bouwmeester, H., Beyer, P., and Al-Babili, S. (2012) The path from beta-carotene to carlactone, a strigolactone-like plant hormone. Science. 335: 1348-1351.

Balzergue, C., Puech-Pags, V., Bécard, G., and Rochange, S.F. (2011) The regulation of arbuscular mycorrhizal symbiosis by phosphate in pea involves early and systemic signalling events. J Exp Bot. 62: 1049-1060.

Banda, J., Bellande, K., von Wangenheim, D., Goh, T., Guyomarc'h, S., Laplaze, L., and Bennett, M.J. (2019) Lateral root formation in Arabidopsis: A well-ordered LRexit. Trends Plant Sci. 24: 826-839.

Barciszewska-Pacak, M., Milanowska, K., Knop, K., Bielewicz, D., Nuc, P., Plewka, P., Pacak, A.M., Vazquez, F., Karlowski, W., Jarmolowski, A., and SzweykowskaKulinska, Z. (2015) Arabidopsis microRNA expression regulation in a wide range of abiotic stress responses. Front Plant Sci. 6: 410.

Barker, S.J., Tagu, D., and Delp, G. (1998) Regulation of root and fungal morphogenesis in mycorrhizal symbioses. Plant Physiol. 116: 1201-1207.

Bates, D., Mächler, M., Bolker, B.M., and Walker, S.C. (2015) Fitting linear mixed-effects models using Ime4. J Stat Softw. 67: 1-48.

Benfey, P.N., and Chua, N.H. (1990) The cauliflower mosaic virus 35S promoter: Combinatorial regulation of transcription in plants. Science. 250: 959-966.

Benková, E., Michniewicz, M., Sauer, M., Teichmann, T., Seifertová, D., Jürgens, G., and Friml, J. (2003) Local, efflux-dependent auxin gradients as a common module for plant organ formation. Cell. 115: 591-602.

Bertani, G. (1951) Studies on lysogenesis. I. The mode of phage liberation by lysogenic Escherichia coli. J Bacteriol. 62: 293-300.

Bhaskara, G.B., Nguyen, T.T., and Verslues, P.E. (2012) Unique drought resistance functions of the highly ABA-induced clade a protein phosphatase 2Cs. Plant Physiol. 160: 379-395.

Bitas, V., McCartney, N., Li, N., Demers, J., Kim, J.-E., Kim, H.-S., Brown, K.M., and Kang, S. (2015) Fusarium oxysporum volatiles enhance plant growth via affecting auxin transport and signaling. Front Microbiol. 6: 1248. 
Borsani, O., Zhu, J., Verslues, P.E., Sunkar, R., and Zhu, J.K. (2005) Endogenous siRNAs derived from a pair of natural cis-antisense transcripts regulate salt tolerance in Arabidopsis. Cell. 123: 1279-1291.

Bourdais, G., Burdiak, P., Gauthier, A., Nitsch, L., Salojärvi, J., Rayapuram, C., Idänheimo, N., Hunter, K., Kimura, S., Merilo, E., Vaattovaara, A., Oracz, K., Kaufholdt, D., Pallon, A., Anggoro, D.T., Glów, D., Lowe, J., Zhou, J., Mohammadi, O., et al. (2015) Large-scale phenomics identifies primary and fine-tuning roles for CRKs in responses related to oxidative stress. PLOS Genet. 11: e1005373.

Brady, S.M., Orlando, D.A., Lee, J.Y., Wang, J.Y., Koch, J., Dinneny, J.R., Mace, D., Ohler, U., and Benfey, P.N. (2007) A high-resolution root spatiotemporal map reveals dominant expression patterns. Science. 318: 801-806.

Brady, S.M., Sarkar, S.F., Bonetta, D., and McCourt, P. (2003) The ABSCISIC ACID INSENSITIVE $3(A B I 3)$ gene is modulated by farnesylation and is involved in auxin signaling and lateral root development in Arabidopsis. Plant J. 34: 67-75.

Buchanan, B.B., Gruissem, W., and Jones, R.L. (2000) The cell wall. In Bio-Chemistry and Molecular Biology of Plants. pp. 52-89 American Society of Plant Physiologists.

Buer, C.S., Wasteneys, G.O., and Masle, J. (2003) Ethylene modulates root-wave responses in Arabidopsis. Plant Physiol. 132: 1085-1096.

Burdiak, P., Rusaczonek, A., Witoń, D., Głów, D., and Karpiński, S. (2015) Cysteine-rich receptor-like kinase CRK5 as a regulator of growth, development, and ultraviolet radiation responses in Arabidopsis thaliana. J Exp Bot. 66: 3325-3337.

Cai, X.T., Xu, P., Zhao, P.X., Liu, R., Yu, L.H., and Xiang, C. Bin (2014) Arabidopsis ERF109 mediates cross-talk between jasmonic acid and auxin biosynthesis during lateral root formation. Nat Commun. 5: 1-13.

Califar, B., Sng, N.J., Zupanska, A., Paul, A.L., and Ferl, R.J. (2020) Root skewingassociated genes impact the spaceflight response of Arabidopsis thaliana. Front Plant Sci. 11: 239.

Cao, Y., Tanaka, K., Nguyen, C.T., and Stacey, G. (2014) Extracellular ATP is a central signaling molecule in plant stress responses. Curr Opin Plant Biol. 20: 82-87.

Carpita, N.C., and Gibeaut, D.M. (1993) Structural models of primary cell walls in flowering plants: Consistency of molecular structure with the physical properties of the walls during growth. Plant J. 3: 1-30.

Casimiro, I., Beeckman, T., Graham, N., Bhalerao, R., Zhang, H., Casero, P., Sandberg, G., and Bennett, M.J. (2003) Dissecting Arabidopsis lateral root development. Trends Plant Sci. 8: 165-171. 
Casimiro, I., Marchant, A., Bhalerao, R.P., Beeckman, T., Dhooge, S., Swarup, R., Graham, N., Inzé, D., Sandberg, G., Casero, P.J., and Bennett, M. (2001) Auxin transport promotes Arabidopsis lateral root initiation. Plant Cell. 13: 843-852.

Chan, Z. (2012) Expression profiling of ABA pathway transcripts indicates crosstalk between abiotic and biotic stress responses in Arabidopsis. Genomics. 100: 110115.

Chang, S., Puryear, J., and Cairney, J. (1993) A simple and efficient method for isolating RNA from pine trees. Plant Mol Biol Report. 11: 113-116.

Chen, S., Zhou, Y., Chen, Y., and Gu, J. (2018) fastp: an ultra-fast all-in-one FASTQ preprocessor. Bioinformatics. 34: i884-i890.

Cheng, Y., Dai, X., and Zhao, Y. (2006) Auxin biosynthesis by the YUCCA flavin monooxygenases controls the formation of floral organs and vascular tissues in Arabidopsis. Genes Dev. 20: 1790-1799.

Chong, J., Wishart, D.S., and Xia, J. (2019) Using MetaboAnalyst 4.0 for comprehensive and integrative metabolomics data analysis. Curr Protoc Bioinforma. 68.

Clough, S.J., and Bent, A.F. (1998) Floral dip: A simplified method for Agrobacteriummediated transformation of Arabidopsis thaliana. Plant J. 16: 735-743.

Correa-Aragunde, N., Foresi, N., Delledonne, M., and Lamattina, L. (2013) Auxin induces redox regulation of ascorbate peroxidase 1 activity by $S$ nitrosylation/denitrosylation balance resulting in changes of root growth pattern in Arabidopsis. J Exp Bot. 64: 3339-3349.

Czechowski, T., Stitt, M., Altmann, T., Udvardi, M.K., and Scheible, W.-R. (2005) Genomewide identification and testing of superior reference genes for transcript normalization in Arabidopsis. Plant Physiol. 139: 5-17.

Davis, E.M., and Croteau, R. (2000) Cyclization Enzymes in the Biosynthesis of Monoterpenes, Sesquiterpenes, and Diterpenes. In Leeper F.J., Vederas J.C. (Eds) Biosynthesis. Topics in Current Chemistry, Vol 209. pp. 53-95 Springer, Berlin, Heidelberg.

Ditengou, F.A., Müller, A., Rosenkranz, M., Felten, J., Lasok, H., van Doorn, M.M., Legué, V., Palme, K., Schnitzler, J.-P., and Polle, A. (2015) Volatile signalling by sesquiterpenes from ectomycorrhizal fungi reprogrammes root architecture. Nat Commun. 6: 6279.

Ditengou, F.A., Teale, W.D., Kochersperger, P., Flittner, K.A., Kneuper, I., Van Der Graaff, E., Nziengui, H., Pinosa, F., Li, X., Nitschke, R., Laux, T., and Palme, K. (2008) Mechanical induction of lateral root initiation in Arabidopsis thaliana. Proc Natl Acad Sci U S A. 105: 18818-18823. 
Dolan, L., Janmaat, K., Willemsen, V., Linstead, P., Poethig, S., Roberts, K., and Scheres, B. (1993) Cellular organisation of the Arabidopsis thaliana root. Development. 119: 71-84.

Dreher, K., and Callis, J. (2007) Ubiquitin, hormones and biotic stress in plants. Ann Bot. 99: 787-822.

Druebert, C. (2011) Funktionelle charakterisierung von apoplastischen proteinen in Brassica napus und Arabidopsis thaliana nach infektion mit Verticillium longisporum. Georg-August-University, Germany.

Du, Y., and Scheres, B. (2018) Lateral root formation and the multiple roles of auxin. J Exp Bot. 69: 155-167.

Dubrovsky, J.G., Napsucialy-Mendivil, S., Duclercq, J., Cheng, Y., Shishkova, S., Ivanchenko, M.G., Friml, J., Murphy, A.S., and Benková, E. (2011) Auxin minimum defines a developmental window for lateral root initiation. New Phytol. 191: 970983.

Dubrovsky, J.G., and Rost, T.L. (2012) Pericycle. In ELS, (Ed.). John Wiley \& Sons, Ltd, Chichester, UK.

Dubrovsky, J.G., Sauer, M., Napsucialy-Mendivil, S., Ivanchenko, M.G., Friml, J., Shishkova, S., Celenza, J., and Benková, E. (2008) Auxin acts as a local morphogenetic trigger to specify lateral root founder cells. Proc Natl Acad Sci U S A. 105: 8790-8794.

Dunwell, J.M., Gibbings, J.G., Mahmood, T., and Saqlan Naqvi, S.M. (2008) Germin and germin-like proteins: evolution, structure, and function. CRC Crit Rev Plant Sci. 27: 342-375.

Edwards, K., Johnstone, C., and Thompson, C. (1991) A simple and rapid method for the preparation of plant genomic DNA for PCR analysis. Nucleic Acids Res. 19: 1349.

Effmert, U., Kalderás, J., Warnke, R., and Piechulla, B. (2012) Volatile mediated interactions between bacteria and fungi in the soil. J Chem Ecol. 38: 665-703.

Engler, C., Kandzia, R., and Marillonnet, S. (2008) A One Pot, One Step, Precision Cloning Method with High Throughput Capability. PLoS One. 3: e3647.

Etchells, J.P., Provost, C.M., and Turner, S.R. (2012) Plant vascular cell division is maintained by an interaction between PXY and ethylene signalling. PLoS Genet. 8: e1002997.

Falik, O., Mordoch, Y., Quansah, L., Fait, A., and Novoplansky, A. (2011) Rumor Has It...: Relay Communication of Stress Cues in Plants. PLoS One. 6: e23625. 
Felten, J., Kohler, A., Morin, E., Bhalerao, R.P., Palme, K., Martin, F., Ditengou, F.A., and Legué, V. (2009) The ectomycorrhizal fungus Laccaria bicolor stimulates lateral root formation in poplar and Arabidopsis through auxin transport and signaling. Plant Physiol. 151: 1991-2005.

Finlay, R.D. (2008) Ecological aspects of mycorrhizal symbiosis: with special emphasis on the functional diversity of interactions involving the extraradical mycelium. $J$ Exp Bot. 59: 1115-1126.

Floerl, S., Majcherczyk, A., Possienke, M., Feussner, K., Tappe, H., Gatz, C., Feussner, I., Kües, U., and Polle, A. (2012) Verticillium longisporum infection affects the leaf apoplastic proteome, metabolome, and cell wall properties in Arabidopsis thaliana. PLoS One. 7: e31435.

Fortin, M.-C., Pierce, F.J., and Poff, K.L. (1989) The pattern of secondary root formation in curving roots of Arabidopsis thaliana (L.) Heynh. Plant, Cell Environ. 12: 337-339.

Fox, J., and Weisberg, S. (2019) An R Companion to Applied Regression, Third Edition. Saga Publications.

Frugis, G., and Chua, N.-H. (2002) Ubiquitin-mediated proteolysis in plant hormone signal transduction. Trends Cell Biol. 12: 308-311.

Fujita, M., Fujita, Y., Noutoshi, Y., Takahashi, F., Narusaka, Y., Yamaguchi-Shinozaki, K., and Shinozaki, K. (2006) Crosstalk between abiotic and biotic stress responses: a current view from the points of convergence in the stress signaling networks. Curr Opin Plant Biol. 9: 436-442.

Fujita, Y., Nakashima, K., Yoshida, T., Katagiri, T., Kidokoro, S., Kanamori, N., Umezawa, T., Fujita, M., Maruyama, K., Ishiyama, K., Kobayashi, M., Nakasone, S., Yamada, K., Ito, T., Shinozaki, K., and Yamaguchi-Shinozaki, K. (2009) Three SnRK2 protein kinases are the main positive regulators of abscisic acid signaling in response to water stress in Arabidopsis. Plant Cell Physiol. 50: 2123-2132.

Fukaki, H., Tameda, S., Masuda, H., and Tasaka, M. (2002) Lateral root formation is blocked by a gain-of-function mutation in the solitary-root/IAA14 gene of Arabidopsis. Plant J. 29: 153-168.

Fukaki, H., Taniguchi, N., and Tasaka, M. (2006) PICKLE is required for SOLITARYROOT/IAA14-mediated repression of ARF7 and ARF19 activity during Arabidopsis lateral root initiation. Plant J. 48: 380-389.

Fukaki, H., and Tasaka, M. (2009) Hormone interactions during lateral root formation. Plant Mol Biol. 69: 437-449. 
García-Gómez, P., Almagro, G., Sánchez-López, Á.M., Bahaji, A., Ameztoy, K., RicarteBermejo, A., Baslam, M., Antolín, M.C., Urdiain, A., López-Belchi, M.D., LópezGómez, P., Morán, J.F., Garrido, J., Muñoz, F.J., Baroja-Fernández, E., and PozuetaRomero, J. (2019) Volatile compounds other than $\mathrm{CO}_{2}$ emitted by different microorganisms promote distinct posttranscriptionally regulated responses in plants. Plant Cell Environ. 42: 1729-1746.

Garnica-Vergara, A., Barrera-Ortiz, S., Muñoz-Parra, E., Raya-González, J., MéndezBravo, A., Macías-Rodríguez, L., Ruiz-Herrera, L.F., and López-Bucio, J. (2016) The volatile 6-pentyl-2 $H$-pyran-2-one from Trichoderma atroviride regulates Arabidopsis thaliana root morphogenesis via auxin signaling and ETHYLENE INSENSITIVE 2 functioning. New Phytol. 209: 1496-1512.

Goh, T., Joi, S., Mimura, T., and Fukaki, H. (2012) The establishment of asymmetry in Arabidopsis lateral root founder cells is regulated by LBD16/ASL18 and related LBD/ASL proteins. Development. 139: 883-893.

Gonzalez-Guzman, M., Apostolova, N., Bellés, J.M., Barrero, J.M., Piqueras, P., Ponce, M.R., Micol, J.L., Serrano, R., and Rodríguez, P.L. (2002) The short-chain alcohol dehydrogenase $A B A 2$ catalyzes the conversion of xanthoxin to abscisic aldehyde. Plant Cell. 14: 1833-1846.

Grennan, A.K. (2008) Arabidopsis MicroRNAs. Plant Physiol. 146: 3-4.

Guo, H.-S., Xie, Q., Fei, J.-F., and Chua, N.-H. (2005) MicroRNA directs mrna cleavage of the transcription factor NAC1 to downregulate auxin signals for Arabidopsis lateral root development. Plant Cell. 17: 1376-1386.

Guo, Y., Ghirardo, A., Weber, B., Schnitzler, J.P., Philipp Benz, J., and Rosenkranz, M. (2019) Trichoderma species differ in their volatile profiles and in antagonism toward ectomycorrhiza Laccaria bicolor. Front Microbiol. 10:891.

Ham, B.K., Li, G., Kang, B.H., Zeng, F., and Lucas, W.J. (2012) Overexpression of Arabidopsis plasmodesmata germin-like proteins disrupts root growth and development. Plant Cell. 24: 3630-3648.

Hao, H.-T., Zhao, X., Shang, Q.-H., Wang, Y., Guo, Z.-H., Zhang, Y.-B., Xie, Z.-K., and Wang, R.-Y. (2016) Comparative digital gene expression analysis of the Arabidopsis response to volatiles emitted by Bacillus amyloliquefaciens. PLoS One. 11: e0158621.

Hartley, J.L., Temple, G.F., and Brasch, M.A. (2000) DNA cloning using in vitro sitespecific recombination. Genome Res. 10: 1788-1795.

Hewage, K.A.H., Yang, J., Wang, D., Hao, G., Yang, G., and Zhu, J. (2020) Chemical manipulation of abscisic acid signaling: $A$ new approach to abiotic and biotic stress management in agriculture. Adv Sci. 2001265. 
Hiraga, S., Sasaki, K., Ito, H., Ohashi, Y., and Matsui, H. (2001) A large family of class III plant peroxidases. Plant Cell Physiol. 42: 462-468.

Hobbie, E.A. (2006) Carbon allocation to ectomycorrhizal fungi correlates with belowground allocation in culture studies. Ecology. 87: 563-569.

Hothorn, T., Bretz, F., and Westfall, P. (2008) Simultaneous inference in general parametric models. Biometrical J. 50: 346-363.

Houston, K., Tucker, M.R., Chowdhury, J., Shirley, N., and Little, A. (2016) The plant cell wall: a complex and dynamic structure as revealed by the responses of genes under stress conditions. Front Plant Sci. 7: 984.

Huang, W., Gfeller, V., and Erb, M. (2019) Root volatiles in plant-plant interactions II: Root volatiles alter root chemistry and plant-herbivore interactions of neighbouring plants. Plant Cell Environ. 42: 1964-1973.

Hung, R., Lee, S., and Bennett, J.W. (2013) Arabidopsis thaliana as a model system for testing the effect of Trichoderma volatile organic compounds. Fungal Ecol. 6: 1926.

Idänheimo, N., Gauthier, A., Salojärvi, J., Siligato, R., Brosché, M., Kollist, H., Mähönen, A.P., Kangasjärvi, J., and Wrzaczek, M. (2014) The Arabidopsis thaliana cysteinerich receptor-like kinases CRK6 and CRK7 protect against apoplastic oxidative stress. Biochem Biophys Res Commun. 445: 457-462.

Ishikawa, S., Maekawa, M., Arite, T., Onishi, K., Takamure, I., and Kyozuka, J. (2005) Suppression of tiller bud activity in tillering dwarf mutants of rice. Plant Cell Physiol. 46.

Ivanchenko, M.G., Muday, G.K., and Dubrovsky, J.G. (2008) Ethylene auxin interactions regulate lateral root initiation and emergence in Arabidopsis thaliana. Plant J. 55: 335-347.

Jia, L., Bai, J., Guan, D., Sun, K., Jiao, Q., and Feng, H. (2016) Extracellular ATP: A potential molecule regulating the defence response of plants to biotic stresses. Review. Plant Prot Sci. 52: 221-228.

Jiang, H., Tang, B., Xie, Z., Nolan, T., Ye, H., Song, G., Walley, J., and Yin, Y. (2019) GSK3like kinase BIN2 phosphorylates RD26 to potentiate drought signaling in Arabidopsis. Plant J. 100: 923-937.

Jiang, L., Jian, H., Qian, J., Sun, Z., Wei, Z., Chen, X., and Cao, S. (2011) MAX4 gene is involved in the regulation of low inorganic phosphate stress responses in Arabidopsis thaliana. Acta Physiol Plant. 33: 867-875. 
Johnson, X., Brcich, T., Dun, E.A., Goussot, M., Haurogné, K., Beveridge, C.A., and Rameau, C. (2006) Branching genes are conserved across species. Genes controlling a novel signal in pea are coregulated by other long-distance signals. Plant Physiol. 142: 1014-1026.

Jung, J.K.H., and McCouch, S. (2013) Getting to the roots of it: genetic and hormonal control of root architecture. Front Plant Sci. 4: 186.

Kaling, M., Schmidt, A., Moritz, F., Rosenkranz, M., Witting, M., Kasper, K., Janz, D., Schmitt-Kopplin, P., Schnitzler, J.-P., and Polle, A. (2018) Mycorrhiza-triggered transcriptomic and metabolomic networks impinge on herbivore fitness. Plant Physiol. 176: 2639-2656.

Kapulnik, Y., Delaux, P.M., Resnick, N., Mayzlish-Gati, E., Wininger, S., Bhattacharya, C., Séjalon-Delmas, N., Combier, J.P., Bécard, G., Belausov, E., Beeckman, T., Dor, E., Hershenhorn, J., and Koltai, H. (2011a) Strigolactones affect lateral root formation and root-hair elongation in Arabidopsis. Planta. 233: 209-216.

Kapulnik, Y., Resnick, N., Mayzlish-Gati, E., Kaplan, Y., Wininger, S., Hershenhorn, J., and Koltai, H. (2011b) Strigolactones interact with ethylene and auxin in regulating roothair elongation in Arabidopsis. J Exp Bot. 62: 2915-2924.

Karimi, M., Inzé, D., and Depicker, A. (2002) Gateway vectors for Agrobacteriummediated plant transformation. Trends Plant Sci. 7: 193-195.

Katzen, F. (2007) Gateway ${ }^{\circledR}$ recombinational cloning: a biological operating system. Expert Opin Drug Discov. 2: 571-589.

Kessler, A., and Baldwin, I.T. (2001) Defensive function of herbivore-induced plant volatile emissions in nature. Science. 291: 2141-2144.

Kottb, M., Gigolashvili, T., Großkinsky, D.K., and Piechulla, B. (2015) Trichoderma volatiles effecting Arabidopsis: from inhibition to protection against phytopathogenic fungi. Front Microbiol. 6: 995.

Kushiro, T., Okamoto, M., Nakabayashi, K., Yamagishi, K., Kitamura, S., Asami, T., Hirai, N., Koshiba, T., Kamiya, Y., and Nambara, E. (2004) The Arabidopsis cytochrome P450 CYP707A encodes ABA 8'-hydroxylases: Key enzymes in ABA catabolism. EMBO J. 23: 1647-1656.

Kushwah, S., Jones, A.M., and Laxmi, A. (2011) Cytokinin interplay with ethylene, auxin, and glucose signaling controls Arabidopsis seedling root directional growth. Plant Physiol. 156: 1851-1866.

Kutz, A., Müller, A., Hennig, P., Kaiser, W.M., Piotrowski, M., and Weiler, E.W. (2002) A role for nitrilase 3 in the regulation of root morphology in sulphur-starving Arabidopsis thaliana. Plant J. 30: 95-106. 
Lamesch, P., Dreher, K., Swarbreck, D., Sasidharan, R., Reiser, L., and Huala, E. (2010) Using The Arabidopsis Information Resource (TAIR) to find information about Arabidopsis genes. Curr Protoc Bioinforma. 30: 1.11.1-1.11.51.

Langmead, B., and Salzberg, S.L. (2012) Fast gapped-read alignment with Bowtie 2. Nat Methods. 9: 357-359.

Lanza, M., Garcia-Ponce, B., Castrillo, G., Catarecha, P., Sauer, M., Rodriguez-Serrano, M., Páez-García, A., Sánchez-Bermejo, E., Tc, M., Leo del Puerto, Y., Sandalio, L.M., Paz-Ares, J., and Leyva, A. (2012) Role of actin cytoskeleton in brassinosteroid signaling and in its integration with the auxin response in plants. Dev Cell. 22: 12751285.

Laskowski, M., Grieneisen, V.A., Hofhuis, H., Hove, C.A. ten, Hogeweg, P., Marée, A.F.M., and Scheres, B. (2008) Root system architecture from coupling cell shape to auxin transport. PLoS Biol. 6: e307.

Laskowski, M.J., Williams, M.E., Nusbaum, H.C., and Sussex, I.M. (1995) Formation of lateral root meristems is a two-stage process. Development. 121.

Lau, O.S., and Deng, X.W. (2012) The photomorphogenic repressors COP1 and DET1: 20 years later. Trends Plant Sci. 17: 584-593.

Lazzarotto, F., Turchetto-Zolet, A.C., and Margis-Pinheiro, M. (2015) Revisiting the nonanimal peroxidase superfamily. Trends Plant Sci. 20: 807-813.

Lee, H.W., Kim, N.Y., Lee, D.J., and Kim, J. (2009) LBD18/ASL20 regulates lateral root formation in combination with LBD16/ASL18 downstream of ARF7 and ARF19 in Arabidopsis. Plant Physiol. 151: 1377-1389.

Lee, S., Behringer, G., Hung, R., and Bennett, J. (2019) Effects of fungal volatile organic compounds on Arabidopsis thaliana growth and gene expression. Fungal Ecol. 37: $1-9$.

Lee, S., Yap, M., Behringer, G., Hung, R., and Bennett, J.W. (2016) Volatile organic compounds emitted by Trichoderma species mediate plant growth. Fungal Biol Biotechnol. 3: 7.

Lewis, D.R., Negi, S., Sukumar, P., and Muday, G.K. (2011) Ethylene inhibits lateral root development, increases IAA transport and expression of PIN3 and PIN7 auxin efflux carriers. Development. 138: 3485-3495.

$\mathrm{Li}, \mathrm{J.}$, and Jia, H. (2013a) Hydrogen peroxide is involved in cGMP modulating the lateral root development of Arabidopsis thaliana. Plant Signal Behav. 8: e25052.

Li, J., and Jia, H. (2013b) cGMP modulates Arabidopsis lateral root formation through regulation of polar auxin transport. Plant Physiol Biochem. 66: 105-117. 
Li, N., Sun, L., Zhang, L., Song, Y., Hu, P., Li, C., and Hao, F.S. (2015) AtrbohD and AtrbohF negatively regulate lateral root development by changing the localized accumulation of superoxide in primary roots of Arabidopsis. Planta. 241: 591-602.

Liu, J., Novero, M., Charnikhova, T., Ferrandino, A., Schubert, A., Ruyter-Spira, C., Bonfante, P., Lovisolo, C., Bouwmeester, H.J., and Cardinale, F. (2013) Carotenoid cleavage dioxygenase 7 modulates plant growth, reproduction, senescence, and determinate nodulation in the model legume Lotus japonicus. J Exp Bot. 64: 19671981.

Love, M.I., Huber, W., and Anders, S. (2014) Moderated estimation of fold change and dispersion for RNA-seq data with DESeq2. Genome Biol. 15: 550.

Lu, K., Liang, S., Wu, Z., Bi, C., Yu, Y.-T., Wang, X.-F., and Zhang, D.-P. (2016) Overexpression of an Arabidopsis cysteine-rich receptor-like protein kinase, CRK5, enhances abscisic acid sensitivity and confers drought tolerance. J Exp Bot. 67: 5009-5027.

Lucas, M., Godin, C., Jay-Allemand, C., and Laplaze, L. (2008) Auxin fluxes in the root apex co-regulate gravitropism and lateral root initiation. J Exp Bot. 59: 55-66.

Luo, Z.-B., Janz, D., Jiang, X., Göbel, C., Wildhagen, H., Tan, Y., Rennenberg, H., Feussner, I., and Polle, A. (2009a) Upgrading root physiology for stress tolerance by ectomycorrhizas: insights from metabolite and transcriptional profiling into reprogramming for stress anticipation. Plant Physiol. 151: 1902-1917.

Luo, Z.-B., Li, K., Jiang, X., and Polle, A. (2009b) Ectomycorrhizal fungus (Paxillus involutus) and hydrogels affect performance of Populus euphratica exposed to drought stress. Ann For Sci. 66: 106-106.

Ma, F., Wang, L., Li, J., Samma, M.K., Xie, Y., Wang, R., Wang, J., Zhang, J., and Shen, W. (2014) Interaction between $\mathrm{HY} 1$ and $\mathrm{H}_{2} \mathrm{O}_{2}$ in auxin-induced lateral root formation in Arabidopsis. Plant Mol Biol. 85: 49-61.

Ma, L., Zhang, H., Sun, L., Jiao, Y., Zhang, G., Miao, C., and Hao, F. (2012) NADPH oxidase AtrbohD and AtrbohF function in ROS-dependent regulation of $\mathrm{Na}^{+} / \mathrm{K}^{+}$homeostasis in Arabidopsis under salt stress. J Exp Bot. 63: 305-317.

Malamy, J.E., and Benfey, P.N. (1997) Organization and cell differentiation in lateral roots of Arabidopsis thaliana. Development. 124: 33-44.

Manzano, C., Pallero-Baena, M., Casimiro, I., De Rybel, B., Orman-Ligeza, B., Van Isterdael, G., Beeckman, T., Draye, X., Casero, P., and del Pozo, J.C. (2014) The emerging role of reactive oxygen species signaling during lateral root development. Plant Physiol. 165: 1105-1119. 
Manzano, C., Ramirez-Parra, E., Casimiro, I., Otero, S., Desvoyes, B., De Rybel, B., Beeckman, T., Casero, P., Gutierrez, C., and del Pozo, J.C. (2012) Auxin and epigenetic regulation of $S K P 2 B$, an F-box that represses lateral root formation. Plant Physiol. 160: 749-762.

Marhavý, P., Vanstraelen, M., De Rybel, B., Zhaojun, D., Bennett, M.J., Beeckman, T., and Benková, E. (2013) Auxin reflux between the endodermis and pericycle promotes lateral root initiation. EMBO J. 32: 149-158.

Marquez-Garcia, B., Njo, M., Beeckman, T., Goormachtig, S., and Foyer, C.H. (2014) A new role for glutathione in the regulation of root architecture linked to strigolactones. Plant, Cell Environ. 37: 488-498.

Migliaccio, F., Fortunati, A., and Tassone, P. (2009) Arabidopsis root growth movements and their symmetry. Plant Signal Behav. 4: 183-190.

Miloradovic van Doorn, M., Merl-Pham, J., Ghirardo, A., Fink, S., Polle, A., Schnitzler, J., and Rosenkranz, M. (2020) Root isoprene formation alters lateral root development. Plant Cell Environ. 43: 2207-2223.

Minerdi, D., Bossi, S., Maffei, M.E., Gullino, M.L., and Garibaldi, A. (2011) Fusarium oxysporum and its bacterial consortium promote lettuce growth and expansin A5 gene expression through microbial volatile organic compound (MVOC) emission. FEMS Microbiol Ecol. 76: 342-351.

Moreno-Risueno, M.A., Van Norman, J.M., Moreno, A., Zhang, J., Ahnert, S.E., and Benfey, P.N. (2010) Oscillating gene expression determines competence for periodic Arabidopsis root branching. Science. 329: 1306-1311.

Mossor-Pietraszewska, T. (2001) Effect of aluminium on plant growth and metabolism. Acta Biochim Pol. 48: 673-686.

Müller, A., Faubert, P., Hagen, M., zu Castell, W., Polle, A., Schnitzler, J.-P.P., and Rosenkranz, M. (2013a) Volatile profiles of fungi - chemotyping of species and ecological functions. Fungal Genet Biol. 54: 25-33.

Müller, A., Volmer, K., Mishra-Knyrim, M., and Polle, A. (2013b) Growing poplars for research with and without mycorrhizas. Crop Sci Hortic. 4: 332.

Naito, Y., Hino, K., Bono, H., and Ui-Tei, K. (2015) CRISPRdirect: software for designing CRISPR/Cas guide RNA with reduced off-target sites. Bioinformatics. 31: 11201123.

Naznin, H.A., Kiyohara, D., Kimura, M., Miyazawa, M., Shimizu, M., and Hyakumachi, M. (2014) Systemic resistance induced by volatile organic compounds emitted by plant growth-promoting fungi in Arabidopsis thaliana. PLoS One. 9.

Negi, S., Ivanchenko, M.G., and Muday, G.K. (2008) Ethylene regulates lateral root formation and auxin transport in Arabidopsis thaliana. Plant J. 55: 175-187. 
Nelson, D.C., Scaffidi, A., Dun, E.A., Waters, M.T., Flematti, G.R., Dixon, K.W., Beveridge, C.A., Ghisalberti, E.L., and Smith, S.M. (2011) F-box protein MAX2 has dual roles in karrikin and strigolactone signaling in Arabidopsis thaliana. Proc Natl Acad Sci U S A. 108: 8897-8902.

O'Callaghan, D., and Charbit, A. (1990) High efficiency transformation of Salmonella typhimurium and Salmonella typhi by electroporation. MGG Mol Gen Genet. 223: 156-158.

Odell, J.T., Nagy, F., and Chua, N.H. (1985) Identification of DNA sequences required for activity of the cauliflower mosaic virus 35 S promoter. Nature. 313: 810-812.

Okushima, Y., Fukaki, H., Onoda, M., Theologis, A., and Tasaka, M. (2007) ARF7 and ARF19 regulate lateral root formation via direct activation of $L B D / A S L$ genes in Arabidopsis. Plant Cell Online. 19.

Oliva, M., and Dunand, C. (2007) Waving and skewing: how gravity and the surface of growth media affect root development in Arabidopsis. New Phytol. 176: 37-43.

Oliveira, R.A. de C., de Andrade, A.S., Imparato, D.O., de Lima, J.G.S., de Almeida, R.V.M., Lima, J.P.M.S., Pasquali, M.A. de B., and Dalmolin, R.J.S. (2019) Analysis of Arabidopsis thaliana redox gene network indicates evolutionary expansion of class III peroxidase in plants. Sci Rep. 9: 15741.

Orman-Ligeza, B., Parizot, B., de Rycke, R., Fernandez, A., Himschoot, E., Van Breusegem, F., Bennett, M.J., Périlleux, C., Beeckman, T., and Draye, X. (2016) RBOH-mediated ROS production facilitates lateral root emergence in Arabidopsis. Development. 143: 3328-39.

Overvoorde, P., Fukaki, H., and Beeckman, T. (2010) Auxin control of root development. Cold Spring Harb Perspect Biol. 2: a001537-a001537.

Panda, S.K., Baluska, F., and Matsumoto, H. (2009) Aluminum stress signaling in plants. Plant Signal Behav. 4: 592-597.

Paul, A.L., Amalfitano, C.E., and Ferl, R.J. (2012) Plant growth strategies are remodeled by spaceflight. BMC Plant Biol. 12: 232.

Pelagio-Flores, R., Muñoz-Parra, E., Barrera-Ortiz, S., Ortiz-Castro, R., Saenz-Mata, J., Ortega-Amaro, M.A., Jiménez-Bremont, J.F., and López-Bucio, J. (2020) The cysteine-rich receptor-like protein kinase CRK28 modulates Arabidopsis growth and development and influences abscisic acid responses. Planta. 251: 3.

Penuelas, J., Asensio, D., Tholl, D., Wenke, K., Rosenkranz, M., Piechulla, B., and Schnitzler J.P. (2014) Biogenic volatile emissions from the soil. Plant Cell Environ. 37: 1866-1891. 
Péret, B., De Rybel, B., Casimiro, I., Benková, E., Swarup, R., Laplaze, L., Beeckman, T., and Bennett, M.J. (2009) Arabidopsis lateral root development: an emerging story. Trends Plant Sci. 14: 399-408.

Pfaffl, M.W. (2001) A new mathematical model for relative quantification in real-time RT-PCR. Nucleic Acids Res. 29: e45.

R Core Team (2019) R: A language and environment for statistical computing. R Found. Stat. Comput. Vienna, Austria. URL https://www.r-project.org/

Rashotte, A.M., Brady, S.R., Reed, R.C., Ante, S.J., and Muday, G.K. (2000) Basipetal auxin transport is required for gravitropism in roots of Arabidopsis. Plant Physiol. 122: 481-490.

Raya-González, J., Pelagio-Flores, R., and López-Bucio, J. (2012) The jasmonate receptor COI1 plays a role in jasmonate-induced lateral root formation and lateral root positioning in Arabidopsis thaliana. J Plant Physiol. 169: 1348-1358.

Richter, G.L., Monshausen, G.B., Krol, A., and Gilroy, S. (2009) Mechanical stimuli modulate lateral root organogenesis. Plant Physiol. 151: 1855-1866.

Robinson, S.J., and Parkin, I.A.P. (2008) Differential SAGE analysis in Arabidopsis uncovers increased transcriptome complexity in response to low temperature. BMC Genomics. 9: 434.

Roux, S.J. (2012) Root waving and skewing - unexpectedly in micro-g. BMC Plant Biol. 12: 231.

Rueden, C.T., Schindelin, J., Hiner, M.C., DeZonia, B.E., Walter, A.E., Arena, E.T., and Eliceiri, K.W. (2017) ImageJ2: ImageJ for the next generation of scientific image data. BMC Bioinformatics. 18.

Rutherford, R., and Masson, P.H. (1996) Arabidopsis thaliana sku mutant seedlings show exaggerated surface- dependent alteration in root growth vector. Plant Physiol. 111: 987-998.

Ruyter-Spira, C., Kohlen, W., Charnikhova, T., van Zeijl, A., van Bezouwen, L., de Ruijter, N., Cardoso, C., Lopez-Raez, J.A., Matusova, R., Bours, R., Verstappen, F., and Bouwmeester, H. (2011) Physiological effects of the synthetic strigolactone analog GR24 on root system architecture in Arabidopsis: another belowground role for strigolactones? Plant Physiol. 155: 721-734.

Růžička, K., Ljung, K., Vanneste, S., Podhorská, R., Beeckman, T., Friml, J., and Benková, E. (2007) Ethylene regulates root growth through effects on auxin biosynthesis and transport-dependent auxin distribution. Plant Cell. 19: 2197-2212. 
De Rybel, B., Vassileva, V., Parizot, B., Demeulenaere, M., Grunewald, W., Audenaert, D., Van Campenhout, J., Overvoorde, P., Jansen, L., Vanneste, S., Möller, B., Wilson, M., Holman, T., Van Isterdael, G., Brunoud, G., Vuylsteke, M., Vernoux, T., De Veylder, L., Inzé, D., et al. (2010) A novel Aux/IAA28 signaling cascade activates GATA23-dependent specification of lateral root founder cell identity. Curr Biol. 20: 1697-1706.

Saini, S., Sharma, I., Kaur, N., and Pati, P.K. (2013) Auxin: A master regulator in plant root development. Plant Cell Rep. 32: 741-757.

Saito, S., Hirai, N., Matsumoto, C., Ohigashi, H., Ohta, D., Sakata, K., and Mizutani, M. (2004) Arabidopsis CYP707As encode (+)-abscisic acid 8'-hydroxylase, a key enzyme in the oxidative catabolism of abscisic acid. Plant Physiol. 134: 1439-1449.

Sallaud, C., Rontein, D., Onillon, S., Jabès, F., Duffé, P., Giacalone, C., Thoraval, S., Escoffier, C., Herbette, G., Leonhardt, N., Causse, M., and Tissiera, A. (2009) A novel pathway for sesquiterpene biosynthesis from Z,Z-farnesyl pyrophosphate in the wild tomato Solanum habrochaites. Plant Cell. 21: 301-317.

Sambrook, J., and Russell, D.W. (2001) Molecular Cloning: A Laboratory Manual 3rd edition. Cold Spring Harbor Laboratory Press.

Schindelin, J., Arganda-Carreras, I., Frise, E., Kaynig, V., Longair, M., Pietzsch, T., Preibisch, S., Rueden, C., Saalfeld, S., Schmid, B., Tinevez, J.-Y., White, D.J., Hartenstein, V., Eliceiri, K., Tomancak, P., and Cardona, A. (2012) Fiji: an opensource platform for biological-image analysis. Nat Methods. 9: 676-682.

Schwechheimer, C., and Deng, X.W. (2000) The COP/DET/FUS proteins - Regulators of eukaryotic growth and development. Semin Cell Dev Biol. 11: 495-503.

Seo, M., Aoki, H., Koiwai, H., Kamiya, Y., Nambara, E., and Koshiba, T. (2004) Comparative studies on the Arabidopsis Aldehyde Oxidase (AAO) gene family revealed a major role of $A A O 3$ in ABA biosynthesis in seeds. Plant Cell Physiol. 45: 1694-1703.

Serino, G., and Deng, X.-W. (2003) The COP9 signalosome: Regulating plant development through the control of proteolysis. Annu Rev Plant Biol. 54: 165-182.

Shapiguzov, A., Vainonen, J.P., Wrzaczek, M., and Kangasjärvi, J. (2012) ROS-talk - how the apoplast, the chloroplast, and the nucleus get the message through. Front Plant Sci. 3: 292.

Shin, R., Burch, A.Y., Huppert, K.A., Tiwari, S.B., Murphy, A.S., Guilfoyle, T.J., and Schachtman, D.P. (2007) The Arabidopsis transcription factor MYB77 modulates auxin signal transduction. Plant Cell. 19: 2440-2453.

Simmons, C., Soil, D., and Migliaccio, F. (1995) Circumnutation and gravitropism cause root waving in Arabidopsis thaliana. J Exp Bot. 46: 143-150. 
De Smet, I., Lau, S., Voß, U., Vanneste, S., Benjamins, R., Rademacher, E.H., Schlereth, A., De Rybel, B., Vassileva, V., Grunewald, W., Naudts, M., Levesque, M.P., Ehrismann, J.S., Inzé, D., Luschnig, C., Benfey, P.N., Weijers, D., Van Montagu, M.C.E., Bennett, M.J., et al. (2010) Bimodular auxin response controls organogenesis in Arabidopsis. Proc Natl Acad Sci U S A. 107: 2705-2710.

De Smet, I., Signora, L., Beeckman, T., Inzé, D., Foyer, C.H., and Zhang, H. (2003) An abscisic acid-sensitive checkpoint in lateral root development of Arabidopsis. Plant J. 33: 543-555.

De Smet, I., Tetsumura, T., De Rybel, B., dit Frey, N.F., Laplaze, L., Casimiro, I., Swarup, R., Naudts, M., Vanneste, S., Audenaert, D., Inzé, D., Bennett, M.J., and Beeckman, T. (2007) Auxin-dependent regulation of lateral root positioning in the basal meristem of Arabidopsis. Development. 134: 681-690.

De Smet, I., Vassileva, V., De Rybel, B., Levesque, M.P., Grunewald, W., Van Damme, D., Van Noorden, G., Naudts, M., Van Isterdael, G., De Clercq, R., Wang, J.Y., Meuli, N., Vanneste, S., Friml, J., Hilson, P., Jürgens, G., Ingram, G.C., Inzé, D., Benfey, P.N., et al. (2008) Receptor-like kinase ACR4 restricts formative cell divisions in the Arabidopsis root. Science. 322: 594-597.

De Smet, I., Zhang, H., Inzé, D., and Beeckman, T. (2006) A novel role for abscisic acid emerges from underground. Trends Plant Sci. 11: 434-439.

Söderström, B., Finlay, R.D., and Read, D.J. (1988) The structure and function of the vegetative mycelium of ectomycorrhizal plants IV. Qualitative analysis of carbohydrate contents of mycelium interconnecting host plants. New Phytol. 109: 163-166.

Sorefan, K., Booker, J., Haurogné, K., Goussot, M., Bainbridge, K., Foo, E., Chatfield, S., Ward, S., Beveridge, C., Rameau, C., and Leyser, O. (2003) MAX4 and RMS1 are orthologous dioxygenase-like genes that regulate shoot branching in Arabidopsis and pea. Genes Dev. 17: 1469-74.

Soundappan, I., Bennett, T., Morffy, N., Liang, Y., Stanga, J.P., Abbas, A., Leyser, O., and Nelsona, D.C. (2015) SMAX1-LIKE/D53 family members enable distinct MAX2dependent responses to strigolactones and karrikins in Arabidopsis. Plant Cell. 27: 3143-3159.

de Souza, E.M., Granada, C.E., and Sperotto, R.A. (2016) Plant pathogens affecting the establishment of plant-symbiont interaction. Front Plant Sci. 7: 15

Splivallo, R., Fischer, U., Göbel, C., Feussner, I., and Karlovsky, P. (2009) Truffles Regulate plant root morphogenesis via the production of auxin and ethylene. Plant Physiol. 150: 2018-2029 
Splivallo, R., Novero, M., Bertea, C.M., Bossi, S., and Bonfante, P. (2007) Truffle volatiles inhibit growth and induce an oxidative burst in Arabidopsis thaliana. New Phytol. 175: 417-424.

Stepanova, A.N., Hoyt, J.M., Hamilton, A.A., and Alonso, J.M. (2005) A link between ethylene and auxin uncovered by the characterization of two root-specific ethylene-insensitive mutants in Arabidopsis. Plant Cell. 17: 2230-2242.

Stirnberg, P., van de Sande, K., and Leyser, H.M.O. (2002) MAX1 and MAX2 control shoot lateral branching in Arabidopsis. Development. 129: 1131-1141.

Street, I.H., Aman, S., Zubo, Y., Ramzan, A., Wang, X., Shakeel, S.N., Kieber, J.J., and Eric Schaller, G. (2015) Ethylene inhibits cell proliferation of the Arabidopsis root meristem. Plant Physiol. 169: 338-350.

Suhre, K., and Schmitt-Kopplin, P. (2008) MassTRIX: mass translator into pathways. Nucleic Acids Res. 36: W481-W484.

Sun, J., Xu, Y., Ye, S., Jiang, H., Chen, Q., Liu, F., Zhou, W., Chen, R., Li, X., Tietz, O., Wu, X., Cohen, J.D., Palme, K., and Li, C. (2009) Arabidopsis ASA1 is important for jasmonate-mediated regulation of auxin biosynthesis and transport during lateral root formation. Plant Cell. 21: 1495-1511.

Sun, X., Bonfante, P., and Tang, M. (2015) Effect of volatiles versus exudates released by germinating spores of Gigaspora margarita on lateral root formation. Plant Physiol Biochem. 97: 1-10.

Swanson, S., and Gilroy, S. (2010) ROS in plant development. Physiol Plant. 138: 384392.

Swarbreck, S.M., Guerringue, Y., Matthus, E., Jamieson, F.J.C., and Davies, J.M. (2019) Impairment in karrikin but not strigolactone sensing enhances root skewing in Arabidopsis thaliana. Plant J. 98: 607-621.

Swarup, R., Perry, P., Hagenbeek, D., Van Der Straeten, D., Beemster, G.T.S., Sandberg, G., Bhalerao, R., Ljung, K., and Bennett, M.J. (2007) Ethylene upregulates auxin biosynthesis in Arabidopsis seedlings to enhance inhibition of root cell elongation. Plant Cell. 19: 2186-2196.

Thimm, O., Bläsing, O., Gibon, Y., Nagel, A., Meyer, S., Krüger, P., Selbig, J., Müller, L.A., Rhee, S.Y., and Stitt, M. (2004) MAPMAN: A user-driven tool to display genomics data sets onto diagrams of metabolic pathways and other biological processes. Plant J. 37: 914-939.

Tognetti, V.B., Mühlenbock, P., and van Breusegem, F. (2012) Stress homeostasis - the redox and auxin perspective. Plant Cell Environ. 35: 321-333.

Torres, M.A. (2010) ROS in biotic interactions. Physiol Plant. 138: 414-429. 
Tsukagoshi, H., Busch, W., and Benfey, P.N. (2010) Transcriptional regulation of ROS controls transition from proliferation to differentiation in the root. Cell. 143: 606616.

Turner, J.G., Ellis, C., and Devoto, A. (2002) The jasmonate signal pathway. Plant Cell. 14: S153-S164.

Tuskan, G.A., DiFazio, S., Jansson, S., Bohlmann, J., Grigoriev, I., Hellsten, U., Putnam, M., Ralph, S., Rombauts, S., Salamov, A., Schein, J., Sterck, L., Aerts, A., Bhalerao, R.R., Bhalerao, R.P., Blaudez, D., Boerjan, W., Brun, A., Brunner, A., et al. (2006) The genome of black cottonwood, Populus trichocarpa (Torr. \& Gray). Science. 313: 1596-1604.

Ülker, B., Peiter, E., Dixon, D.P., Moffat, C., Capper, R., Bouché, N., Edwards, R., Sanders, D., Knight, H., and Knight, M.R. (2008) Getting the most out of publicly available TDNA insertion lines. Plant J. 56: 665-677.

Ulmasov, T., Murfett, J., Hagen, G., and Guilfoyle, T.J. (1997) Aux/IAA proteins repress expression of reporter genes containing natural and highly active synthetic auxin response elements. Plant Cell. 9: 1963-1971.

Usadel, B., Nagel, A., Thimm, O., Redestig, H., Blaesing, O.E., Palacios-Rojas, N., Selbig, J., Hannemann, J., Piques, M.C., Steinhauser, D., Scheible, W.-R., Gibon, Y., Morcuende, R., Weicht, D., Meyer, S., and Stitt, M. (2005) Extension of the Visualization Tool MapMan to Allow Statistical Analysis of Arrays, Display of Coresponding Genes, and Comparison with Known Responses. Plant Physiol. 138: 1195-1204.

Vaattovaara, A., Brandt, B., Rajaraman, S., Safronov, O., Veidenberg, A., Luklová, M., Kangasjärvi, J., Löytynoja, A., Hothorn, M., Salojärvi, J., and Wrzaczek, M. (2019) Mechanistic insights into the evolution of DUF26-containing proteins in land plants. Commun Biol. 2: 1-18.

Vanzo, E., Ghirardo, A., Merl-Pham, J., Lindermayr, C., Heller, W., Hauck, S.M., Durner, J., and Schnitzler, J.-P. (2014) S-Nitroso-Proteome in poplar leaves in response to acute ozone stress. PLoS One. 9: e106886.

Vanzo, E., Merl-Pham, J., Velikova, V., Ghirardo, A., Lindermayr, C., Hauck, S.M., Bernhardt, J., Riedel, K., Durner, J., and Schnitzler, J.P. (2016) Modulation of protein s-nitrosylation by isoprene emission in poplar. Plant Physiol. 170: 1945-1961.

Velikova, V., Edreva, A., and Loreto, F. (2004) Endogenous isoprene protects Phragmites australis leaves against singlet oxygen. Physiol Plant. 122: 219-225.

Velikova, V., Sharkey, T.D., and Loreto, F. (2012) Stabilization of thylakoid membranes in isoprene-emitting plants reduces formation of reactive oxygen species. Plant Signal Behav. 7: 139-141. 
Vickers, C.E., Gershenzon, J., Lerdau, M.T., and Loreto, F. (2009) A unified mechanism of action for volatile isoprenoids in plant abiotic stress. Nat Chem Biol. 5: 283-291.

Vishwanathan, K., Zienkiewicz, K., Liu, Y., Janz, D., Feussner, I., Polle, A., and Haney, C.H. (2020) Ectomycorrhizal fungi induce systemic resistance against insects on a nonmycorrhizal plant in a CERK1-dependent manner. New Phytol. nph.16715.

Vu, V.Q. (2011) ggbiplot: A ggplot2 based biplot. R Packag. version 0.55. URL http://github.com/vqv/ggbiplot

Wägele, B., Witting, M., Schmitt-Kopplin, P., and Suhre, K. (2012) MassTRIX Reloaded: Combined Analysis and Visualization of Transcriptome and Metabolome Data. PLOS One. 7: e39860.

Waidmann, S., Sarkel, E., and Kleine-Vehn, J. (2020) Same same, but different: growth responses of primary and lateral roots. J Exp Bot. 71: 2397-2411.

Wang, L., Wang, B., Jiang, L., Liu, X., Li, X., Lu, Z., Meng, X., Wang, Y., Smith, S.M., and Lia, J. (2015) Strigolactone signaling in Arabidopsis regulates shoot development by targeting D53-like SMXL repressor proteins for ubiquitination and degradation. Plant Cell. 27: 3128-3142.

Wang, Z.-P., Xing, H.-L., Dong, L., Zhang, H.-Y., Han, C.-Y., Wang, X.-C., and Chen, Q.-J. (2015) Egg cell-specific promoter-controlled CRISPR/Cas9 efficiently generates homozygous mutants for multiple target genes in Arabidopsis in a single generation. Genome Biol. 16: 144.

Wasternack, C. (2007) Jasmonates: An Update on Biosynthesis, Signal Transduction and Action in Plant Stress Response, Growth and Development. Ann Bot. 100: 681-697.

Weigel, D., and Glazebrook, J. (2002) Arabidopsis: A Laboratory Manual. Cold Spring Harbor Laboratory Press, Cold Spring Harbor (N.Y.).

Weikl, F., Ghirardo, A., Schnitzler, J.-P., and Pritsch, K. (2016) Sesquiterpene emissions from Alternaria alternata and Fusarium oxysporum: Effects of age, nutrient availability, and co-cultivation. Sci Rep. 6: 22152.

Werner, S., Polle, A., and Brinkmann, N. (2016) Belowground communication: impacts of volatile organic compounds (VOCs) from soil fungi on other soil-inhabiting organisms. Appl Microbiol Biotechnol. 100: 8651-8665.

Witting, M., Maier, T.V., Garvis, S., and Schmitt-Kopplin, P. (2014) Optimizing a ultrahigh pressure liquid chromatography-time of flight-mass spectrometry approach using a novel sub- $2 \mu \mathrm{m}$ core-shell particle for in depth lipidomic profiling of Caenorhabditis elegans. J Chromatogr A. 1359: 91-99. 
Wrzaczek, M., Brosché, M., Salojärvi, J., Kangasjärvi, S., Idänheimo, N., Mersmann, S., Robatzek, S., Karpiński, S., Karpińska, B., and Kangasjärvi, J. (2010) Transcriptional regulation of the CRK/DUF26 group of Receptor-like protein kinases by ozone and plant hormones in Arabidopsis. BMC Plant Biol. 10: 95.

Wu, J., and Baldwin, I.T. (2010) New insights into plant responses to the attack from insect herbivores. Annu Rev Genet. 44: 1-24.

Xie, X., Zhang, H., and Pare, P. (2009) Sustained growth promotion in Arabidopsis with long-term exposure to the beneficial soil bacterium Bacillus subtilis (GB03). Plant Signal Behav. 4: 948-953.

Xing, H.-L., Dong, L., Wang, Z.-P., Zhang, H.-Y., Han, C.-Y., Liu, B., Wang, X.-C., and Chen, Q.-J. (2014) A CRISPR/Cas9 toolkit for multiplex genome editing in plants. $B M C$ Plant Biol. 14: 327.

Xing, L., Zhao, Y., Gao, J., Xiang, C., and Zhu, J.K. (2016) The ABA receptor PYL9 together with PYL8 plays an important role in regulating lateral root growth. Sci Rep. 6: 113.

Xu, J., Trainotti, L., Li, M., and Varotto, C. (2020) Overexpression of isoprene synthase affects aba- and drought-related gene expression and enhances tolerance to abiotic stress. Int J Mol Sci. 21: 4276.

Xu, P., Zhao, P.-X., Cai, X.-T., Mao, J.-L., Miao, Z.-Q., and Xiang, C.-B. (2020) Integration of jasmonic acid and ethylene into auxin signaling in root development. Front Plant Sci. 11: 271.

Xue, R., and Zhang, B. (2007) Increased endogenous methyl jasmonate altered leaf and root development in transgenic soybean plants. J Genet Genomics. 34: 339-346.

Yamagiwa, Y., Inagaki, Y., Ichinose, Y., Toyoda, K., Hyakumachi, M., and Shiraishi, T. (2011) Talaromyces wortmannii FS2 emits $\beta$-caryphyllene, which promotes plant growth and induces resistance. J Gen Plant Pathol. 77: 336-341.

Yoneyama, Koichi, Kisugi, T., Xie, X., and Yoneyama, Kaori (2013) Chemistry of strigolactones: Why and how do plants produce so many strigolactones? Mol Microb Ecol Rhizosph. 1: 373-379.

Yuan, C., Wang, J., Harrison, A.P., Meng, X., Chen, D., and Chen, M. (2015) Genome-wide view of natural antisense transcripts in Arabidopsis thaliana. DNA Res. 22: 233-243.

Zhang, H., Kim, M.S., Krishnamachari, V., Payton, P., Sun, Y., Grimson, M., Farag, M.A., Ryu, C.M., Allen, R., Melo, I.S., and Paré, P.W. (2007) Rhizobacterial volatile emissions regulate auxin homeostasis and cell expansion in Arabidopsis. Planta. 226: 839-851. 
Zhang, H., Sun, Y., Xie, X., Kim, M.S., Dowd, S.E., and Paré, P.W. (2009) A soil bacterium regulates plant acquisition of iron via deficiency-inducible mechanisms. Plant J. 58: 568-577.

Zhang, H., Xie, X., Kim, M.S., Kornyeyev, D.A., Holaday, S., and Paré, P.W. (2008) Soil bacteria augment Arabidopsis photosynthesis by decreasing glucose sensing and abscisic acid levels in planta. Plant J. 56: 264-273.

Zhao, Y., Xing, L., Wang, X., Hou, Y.-J., Gao, J., Wang, P., Duan, C.-G., Zhu, X., and Zhu, J.K. (2014) The ABA receptor PYL8 promotes lateral root growth by enhancing MYB77-dependent transcription of auxin-responsive genes. Sci Signal. 7: ra53. 


\section{Declaration}

The Arabidopsis metabolome data were provided by Patrizia Thoma under the supervision of Prof. Dr. Jörg-Peter Schnitzler (Helmholtz Zentrum München, Research Unit Environmental Simulation). 


\section{Acknowledgement}

First of all, I would like to thank my supervisor Prof. Dr. Andrea Polle for providing me an excellent opportunity to work on such an interesting topic. Her continuous support during my PhD project, her encouragement to believe in myself and for strengthening my self-confidence in every step and decision.

Furthermore, I would like to thank my second supervisor and collaboration partner Prof. Dr. Jörg-Peter Schnitzler for his support, for his numerous fruitful ideas during the project meetings and for providing the possibility to analyze the metabolomics data in his group. I would additionally like to thank Prof. Dr. Gerhard Braus for being a member of my thesis committee.

I would like to extend my gratitude to Prof. Dr. Ivo Feußner, PD Dr. Till Ischebeck and Prof. Dr. Kai Heimel for being a member of my examination board.

Many thanks to Dr. Corinna Thurow and Prof. Dr. Christiane Gatz for introducing me to the CRISPR/Cas9 cloning system and providing the pBCsGFPEE and pBCsGEE-ROXY11-15BA plasmids along with the V69-primer sequences. I am thankful for their enlightenments through ideas and suggestions, with the molecular cloning protocol and for always being available in case of doubts and questions. Additional thanks for providing the aba2-1 and the aao3-4 mutant lines.

I am thankful to PD Dr. Michael Wrzaczek (University of Helsinki) who provided the CRK mutant library. Additional thanks to Yang Zhao (Shanghai Institutes for Biological Sciences) for providing the pyl8-1 and the myb77-1 mutant lines. Further acknowledgements to Ashley Cannon (University of North Texas) for providing the pyl sextuple mutant.

Thanks to Patrizia Thoma (Helmholtz Zentrum München) for helping me with the metabolomics study. Thanks to Maaria Rosenkranz (Helmholtz Zentrum München) for her significant contributions to the project meetings. 
I thank all members of the department of Forest Botany and Tree Physiology at the Georg-August-Universität Göttingen for supporting my work in every aspect and for being friends and great colleagues for the last four year. Special thanks to Dr. Christian Eckert for his help and support in the molecular work and to Dr. Dennis Janz, for the support in processing of the transcriptome and metabolome data along with the guidance to perform the statistical analyses. My heartfelt acknowledgements to Katharina Ziesing who not only introduced me to the practical and laboratory aspects of my project but also invested numerous hours with or without my assistance at the sterile bench to prepare the experiments. Furthermore, she helped me in the determination of the lateral root densities, of probably thousands of plants. Without her tremendous support, the work would have been much less comprehensive.

I want to thank Merle Fastenrath for her assistance in the laboratory-related issues and Cathrin Leibecke and Monika-Franke Klein for their assistance with climate chamber related issues.

Thanks to Bernd Kopka for fixing IT related problems, especially for rescuing my hard disk during the critical period of thesis writing. Last but not least, I want to thank Nina-Christin Lindemann and Dr. Ulrike Lipka for taking care of the administrative, health and safety concerns.

Special thanks to Ishani Shankar Das for becoming friends during the last year and for her emotional support especially during the last days of my work.

I want to thank the German Foundation (DFG) for funding the poplar communication project DFG (Po 362/20-2).

I want to thank my family who supported me all the way to my doctorate. Without them, this path of study would have been much more difficult. And finally, I want to thank my fiancé Andreas, who always watched my back, especially during the last few hectic months. 